University of Rhode Island

DigitalCommons@URI

Open Access Dissertations

2020

\title{
TAILORING DIGITAL HEALTH TO IMPROVE THE NUTRITION AND HEALTH OF PEOPLE WITH PARKINSON'S DISEASE
}

Dara Lyn LoBuono

University of Rhode Island, dara_lobuono@uri.edu

Follow this and additional works at: https://digitalcommons.uri.edu/oa_diss

\section{Recommended Citation}

LoBuono, Dara Lyn, "TAILORING DIGITAL HEALTH TO IMPROVE THE NUTRITION AND HEALTH OF PEOPLE WITH PARKINSON'S DISEASE" (2020). Open Access Dissertations. Paper 1153.

https://digitalcommons.uri.edu/oa_diss/1153

This Dissertation is brought to you for free and open access by DigitalCommons@URI. It has been accepted for inclusion in Open Access Dissertations by an authorized administrator of DigitalCommons@URI. For more information, please contact digitalcommons-group@uri.edu. 
TAILORING DIGITAL HEALTH TO IMPROVE THE NUTRITION AND HEALTH OF PEOPLE WITH PARKINSON'S DISEASE

\author{
BY \\ DARA LYN LOBUONO
}

\begin{abstract}
A DISSERTATION SUBMITTED IN PARTIAL FULFILLMENT OF THE REQUIREMENTS FOR THE DEGREE OF DOCTOR OF PHILOSOPHY IN BIOLOGICAL AND ENVIRONMENTAL SCIENCES
\end{abstract}

UNIVERSITY OF RHODE ISLAND

2020 
DOCTOR OF PHILOSOPHY IN BIOLOGICAL AND ENVIRONMENTAL SCIENCES

OF

DARA LYN LOBUONO

\section{APPROVED:}

Dissertation Committee:

MAJOR PROFESSOR: Ingrid E. Lofgren

Alison Tovar
Leslie Mahler

Skye N. Leedahl

Nasser H. Zawia

Dean of the Graduate School

UNIVERSITY OF RHODE ISLAND

2020 


\begin{abstract}
Parkinson's disease (PD) is an incurable, neurodegenerative movement disorder, impacting nearly one million Americans. By 2030 the number of people with PD (PwPD) is expected to double and with this growing population, informal caregiver responsibility will also increase. Nutritional status worsens as PD progresses, which impacts cognition, body composition, ability to perform activities of daily living (ADL) and quality of life (QOL), and increases caregiver burden. Nutritional screening and intervention for PwPD can improve health outcomes, but are often excluded from PD treatment plans.
\end{abstract}

Given the negative impact of PD on mobility and increased caregiver burden, digital technology could improve access to health care services for PwPD and their caregivers, making them excellent candidates for digital health. Digital health services (i.e. wearable devices, videoconferencing, phone apps) are used in PD management across many health disciplines, but have not been implemented for nutritional management of PD. Understanding how nutritional status changes overtime for PwPD, and including PwPD and their caregivers in the formative stage is critical to developing effective digital health services. The aim of this body of research is to: 1) describe how the nutrition status of PwPD changes overtime, 2) describe the diet quality and self-reported nutrition concerns of PwPD and their informal caregivers, 3) 
collect formative data around digital health to manage nutrition for PwPD and their caregivers through dyadic interviewing.

The first chapter focuses on tracking the nutritional status of eight PwPD over four years and examines how disease sequelae, conditions that result from PD, may influence nutritional status. Findings reveal that the majority of PwPD were either at "possible-" or "at nutrition-" risk throughout the four years. There was a modest decrease in weight and body mass index. Findings suggest that PwPD can benefit from ongoing nutrition screening throughout the course of PD.

Chapter two evaluates the diet quality via Healthy Eating Index (HEI)2015 scores and self-reported nutrition concerns identified from qualitative interviews of twenty PwPD and their informal caregivers. This chapter also explores if a consistent pattern existed between nutrition concerns coded and diet quality scores of PwPD and caregivers. Mean HEI-2015 scores of PwPD and caregivers translate to an F letter grade and both PwPD and caregivers have intake inconsistent with the Dietary Guidelines for Americans. Participants also have low HEI-2015 component scores for whole grains, fatty acid ratios, and greens \& beans. Qualitative themes specifically around dietary concerns related to PD sequelae include: change in appetite, amount eaten and/or weight, gastrointestinal issues, food-medication management, chewing/swallowing issues, change in taste/smell. No consistent pattern between HEI-2015 scores and self-reported nutrition concerns were detected. 
Findings suggest this population could benefit from nutrition services to better health outcomes.

Chapter 3 examines twenty PwPD's and their caregivers' perception and acceptance of digital health for managing nutrition and health through semi-structured dyadic interviews and questionnaires. This study also evaluates the participants' level of digital competence. Phrases from interviews related to perceptions of digital health were sub-coded into three categories: Perceived Usefulness, Perceived Ease of Use and Awareness of Digital Health. Phrases related to Acceptance of digital were sub-coded into Accept, Neutral or Reject. An Average Dyadic Acceptance Rate for digital health was obtained through averaging the percent of phrases coded as Accept from each interview transcript. To integrate the two data sets, qualitative codes were transformed into variables and compared to digital competence scores. Twenty-five (62.5\%) participants used the internet for at least 5 health-related purposes. The Average Dyadic Acceptance Rate was $54.4 \%$. Dyads rejected digital health devices if they did not see the added benefit. The majority of phrases coded revealed participants found digital health useful, but hard to use, and about half of the phrases coded indicate dyads needed education about existing digital health mediums. Findings suggest dyads are accepting of technology but are not utilizing technology to its full potential. Perceiving technology as hard to use and digital competence scores, implies education is warranted prior to providing a digital nutrition service. 
This body of research supports the need for nutrition screening and services among both PwPD and caregivers, who present with poor diet quality. Findings from this study also suggest more research is needed to figure out how to increase acceptability of digital health among this population. However, low diet quality scores, current technology usage, and perceived usefulness of digital health suggests technology may be a way to increase access to nutrition professionals among the PD community to promote better health outcomes. 


\section{ACKNOWLEDGEMENTS}

I would like to thank my major advisor, Dr. Ingrid Lofgren. Your mentorship and guidance since my Master's degree has been invaluable and has shaped the person I am today. Thank you for consistently supporting me and pushing me to be my best. You have not only made me a better researcher and writer, but have shown me how to be an effective leader and compartmentalize responsibilities when having to wear so many hats! It has been such an honor to complete my $\mathrm{PhD}$ under you and I hope I can be as an amazing advisor and teacher as you are to my students.

I would also like to thank my dissertation committee. To Dr. Alison Tovar, thank you for all of the opportunities you have provided me with over the years to gain experience with collecting dietary data and helping me to strengthen my understanding of mixed-methods research. Dr. Skye Leedahl, it has been such a wonderful opportunity to work with you on so many interesting and exciting projects, including Cyber Seniors. Through your mentorship I have acquired a deeper understanding of older adults, healthcare issues and qualitative research. Dr. Leslie Mahler, I am so grateful for our extensive working relationship both through research and through community outreach efforts. You have helped me to truly understand the cognitive and speech changes people with neurological disorders experience and how this may impact the nutrition education provided. Lastly, I would like to thank my committee chair, Dr. Furong Xu, for her ongoing support with statistical 
analyses, constructive feedback on my writing, and helping me to gain an understanding of physical functioning research.

In addition to my committee members, I would like to recognize all of the undergraduate and graduate students in the Lipid Metabolism lab over the years who helped support this research. In particular, I would like to thank Kyla Shea, who made a substantial contribution in data collection, analyses and visualization. I appreciate your investment and dedication to these projects! I would also like to thank my lab mate, Sarah Dobszewski, and former graduate student, Janette Bedoyan for their support and assistance with data collection. Thank you to all the undergraduate research assistants that have assisted with this project, including: Jayca Payne, Isabelle Masse and Emma Davidson.

I would like to thank the Department of Nutrition and Food Sciences. In particular, the faculty who I have had the opportunity to work closely with as a teaching assistant or as an instructor for the online program. This includes, Cathy English, Jennifer Arts-Salvatore, Kimberly Koness, Brietta Oaks, Maya Vadiveloo, Sarah Larson, and Amanda Missimer. Thank you to all the NFS graduate students, including Amy Moore, Alyssa Abreu, Allisa Georgio, Andrea Ramirez, Kelsey McNulty, Haley Parker, Carolina de Araujo, Ajita Jadhav, Jackie Potvin, Katelyn Fox, Margaret Samson, and Celia Palmer. As well as honorary NFS graduate student, Ashton Foley-Schramm. It has been such an honor to share the journey of graduate school with you all. I would also like to thank my former classmates including Natalie Weisfeld, Jacqueline 
Beatty, and Jade McNamara. Finally, thank you to Valerie Jenkins for her ongoing support.

Additionally, I would like to thank the Department of Gerontology and the Interprofessional Education Practice Initiative for the on-going opportunities you have provided me with. Dr. Phil Clark, Barbara Sweeney, Faith Helm, Christine Ferrone, Allie Morelli, Dr. Brian Quilliam, and Kristin Fratoni - it has been such an honor to work with you all the last couple of years.

Thank you to the Rhode Island Chapter of the American Parkinson's Disease Association (RIAPDA). In particular, thank you to those in the Parkinson's community who participated in these studies, as well as to Mary Ellen Thibodeau for her mentorship. A special, thank you to both those involved with LOUD for Life and the RIAPDA Barrington Support Group. It has been an honor to share your journey and acquire a better understanding of Parkinson's disease.

I would like to thank my friends and family. In particular, thank you, Noereem Mena for being a colleague and best friend. It has been such a journey completing so much graduate school together. Thank you to Indie Rock I, Barbara Farrell, Casey Gambuti and Becca Katz for your unconditional love and support over the years. Finally, thank you to my parents for encouraging me to chase my dreams. 


\section{DEDICATION}

This dissertation is dedicated to my grandmother, Lena Largo

(5/17/1926-12/16/2019), and to my good luck charm, Leanna Rae Ippoliti. 


\section{PREFACE}

This dissertation is presented in manuscript format and contains three chapters that are from two different studies through the University of Rhode Island. Upon completion of the final dissertation submission, three manuscripts will be submitted for publication to the specified journal highlighted on each manuscript title page. Chapter one will be submitted to Movement Disorders, chapter two will be submitted to the Journal of the Academy of Nutrition and Dietetics, and chapter three will be submitted to Health and Social Care in the Community. 


\section{TABLE OF CONTENTS}

Chapter and Title

Page Number

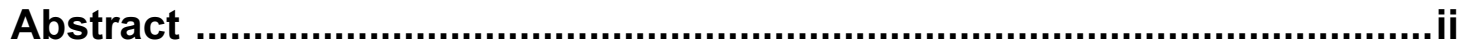

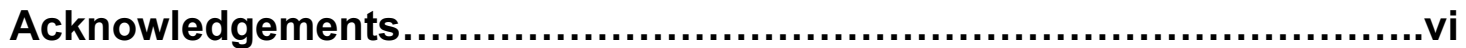

Dedication..............................................................................

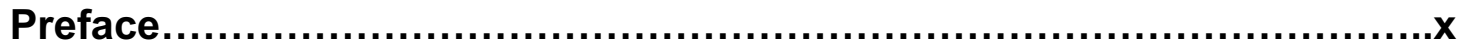

Table of Contents....................................................................

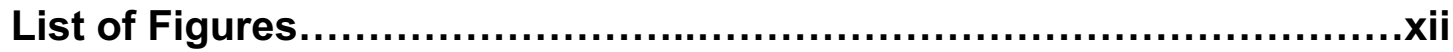

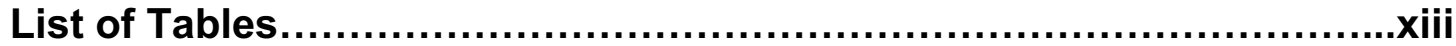

Chapter One

A Comprehensive Longitudinal Nutrition Assessment of People with

Parkinson's Disease Over Four Years...............................................1

Chapter Two:

Diet Quality and Nutrition Concerns of People with Parkinson's Disease and Their Informal Caregivers: A Mixed Methods Study....

\section{Chapter Three:}

Acceptance and Perception of Digital Health for Managing Nutrition in People with Parkinson's Disease and Their Caregivers and Their Digital Competence in the United States: A Mixed-Methods study....

Extended Literature Review ..............................................98

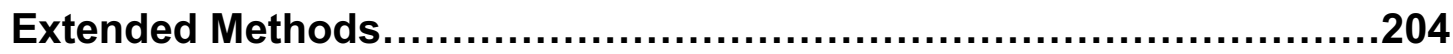

Methodology Appendices....................................................230 


\section{LIST OF FIGURES}

Chapter and Figure Number

Page Number

Chapter One, Figure 1:

Propose Mechanism of How Disease Sequalae Impact Nutrition Status Which

May Further Impact Health Outcome......................................13

Chapter Two, Figure 1:

Flow Chart of Qualitative Data by Major Categories and Sub-Themes.

Chapter Two, Figure 2:

Frequency of PwPD and Caregivers with $\geq 80 \%$ Adequacy and Moderation

Component Scores......................................................... 46 


\section{LIST OF TABLES}

Chapter and Table Number

Page Number

Chapter One, Table 1:

Nutrition Assessment Markers Among PwPD Over 4 Years.

14

Chapter One, Table 2:

Change in Disease Sequelae (Cognition, Physical Function, Swallowing)

Among PwPD Over Four Years.

14

Chapter Two, Table 1:

Descriptive Characteristics of PwPD and Caregivers

47

Chapter Two, Table 2:

Diet Assessment of PwPD and Caregivers

Chapter Two, Table 3:

HEI-2015 Scores of PwPD and Caregivers

Chapter Two, Table 4

Number of Times Nutrition Concerns Related to Parkinson's Disease

Sequelae Were Coded During Dyadic Interviews: A Side-By-Side Comparison

with HEI Scores.

Chapter Three, Table 1

Participant Demographics

Chapter Three, Table 2a

Distribution of Technological Purposes Among PwPD and Caregivers

Reported in Frequencies and Percentages. 
Chapter Three, Table $2 b$

Description of Internet Use for managing Health Among PwPD and Caregivers Reported in Frequencies and Percentages

Chapter Three, Table 3

Themes for Perception of Digital Health Summarized by Number of Phrases

Coded, Percent of Comments, and Number of Dyads Mentioning Perceptions

within Each Category

Chapter Three, Table 4

Those Participants Who Responded Slightly or Strongly Agree to Individual

Digital Competence Questions Among PwPD and Caregivers Reported by

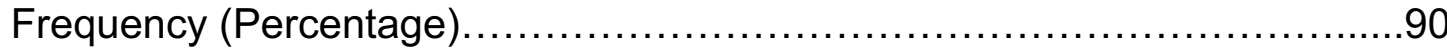

Chapter Three, Table 5

Data Integration: Side by Side Display of Digital Competence Scores

(total scores $(\%)$ ), Acceptance Rates Calculated From

Semi-Structured Interviews. 


\section{CHAPTER ONE}

A Comprehensive Longitudinal Nutrition Assessment of People with Parkinson's Disease Over Four Years

By

Dara L. LoBuono ${ }^{1}$, Alison Tovar², Skye N. Leedahl' ${ }^{3}$, Matthew J. Delmonico ${ }^{4}$, Leslie Mahler ${ }^{5}$, Furong $\mathrm{Xu}^{6}$, Ingrid Lofgren ${ }^{7}$

will be submitted to: Movement Disorders Journal

${ }^{1}$ PhD Candidate, Department of Nutrition and Food Sciences, The University of Rhode Island, Kingston, RI 02881. Email: dara lobuono@uri.edu

${ }^{2}$ Associate Professor, Department of Nutrition and Food Sciences, The University of Rhode Island, Kingston, RI 02881. Email: alison tovar@uri.edu

${ }^{3}$ Associate Professor, Department of Human Development and Family Studies, The University of Rhode Island, RI, 02881. Email:

skyeleedahl@uri.edu

${ }^{4}$ Professor, Department of Kinesiology, The University of Rhode Island, RI 02881. Email: delmonico@uri.edu

${ }^{5}$ Associate Professor, Department of Communicative Disorders, The University of Rhode Island, RI 02811. Email: Imahler@uri.edu

${ }^{6}$ Professor, Department of Kinesiology, The University of Rhode Island, RI 02881. Email: fxu2007@uri.edu

${ }^{7}$ Professor, Department of Nutrition and Food Sciences, The University of Rhode Island, RI, 02881. Email: ingrid lofgren@uri.edu 
A Comprehensive Longitudinal Nutrition Assessment of People with Parkinson's Disease Over Four Years

Authors:

Dara L. LoBuono ${ }^{1}$, Furong $\mathrm{Xu}^{2}$, Alison Tovar ${ }^{1}$, Skye N. Leedahl' ${ }^{3}$, Matthew J. Delmonico ${ }^{2}$, Leslie Mahler ${ }^{4}$, Ingrid E. Lofgren ${ }^{1}$

${ }^{1}$ Department of Nutrition and Food Sciences, ${ }^{2}$ Department of Kinesiology , ${ }^{3}$ Departmentn of Human Development and Family Studies, ${ }^{4}$ Department of Communicative Disorders

\section{Abstract}

Background: Parkinson's disease (PD), a neurodegenerative disease, results in motor and non-motor changes that can impact nutritional status. How nutrition assessment markers change longitudinally has not been examined. The purpose of this study was to track the nutritional status of people with Parkinson's disease (PwPD) over time.

Methods: This study was an observational, longitudinal study examining people with PwPD with assessments analyzed at baseline, year 2 and year 4 . The assessments included: the dietary screening tool (DST), height and weight to calculate body mass index (BMI), fasting glucose and lipid profiles, and blood pressure. A one-way repeated measures ANCOVA compared outcomes variables over time.

Results: Eight PwPD were assessed. The baseline age was $67.1 \pm 4.0$ years and time since diagnosis was $8.1 \pm 7.5$ years. There was no change in mean DST scores overtime $(64.0 \pm 13.8$ vs. $66.4 \pm 8.8$ vs. $64.3 \pm 13.2)$ and majority of participants were at possible or at nutrition risk at each assessment. 
Participants experienced weight declines (176.3 $\pm 29.7 \mathrm{lbs}$ vs. $169.0 \pm 25.5 \mathrm{lbs}$, $\left.\eta_{p}^{2}=0.01\right)$ and BMI $\left(27.6 \mathrm{~kg} / \mathrm{m}^{2}\right.$ vs. $\left.26.6 \pm 2.2, \eta_{\mathrm{p}}{ }^{2}=0.02\right)$ from baseline to year 4. The number of PwPD with elevated glucose $(\geq 100 \mathrm{mg} / \mathrm{dL})$ increased from one to four, but the number of PwPD with suboptimal HDL-C decreased from four to two.

Conclusion: The presence of nutrition risk, experienced weight loss, and changes in biochemical and clinical values, suggest that interdisciplinary intervention strategies may need to be designed and tested in this population.

Key words: Parkinson's disease, nutritional status, diet quality, longitudinal study, cardiometabolic risk 


\section{Introduction}

Parkinson's disease (PD) is an incurable, progressive neurodegenerative disease that traditionally occurs in the second half of life. ${ }^{1}$ Over 900,000 Americans are diagnosed with $\mathrm{PD}^{2,3}$, and that number is expected to double by 2030. ${ }^{3}$ People with PD (PwPD) typically live about 15 after diagnosis and just recently researchers are investigating what post one year from diagnosis. ${ }^{4}$ Parkinson's disease-specific sequelae, conditions that result from PD, impact motor and non-motor function ${ }^{5}$ and worsen as the disease progresses. ${ }^{5}$ Common motor sequelae (slowness of movement, tremors, and balance issues), and common non-motor sequelae (changes in smell and taste, gastrointestinal issues and difficulty swallowing), are all associated with compromised nutritional status. ${ }^{6-8}$ Monitoring these changes overtime provides how nutrition health outcomes among PwPD change overtime in light motor and non-motor sequelae.

While PD sequelae tracking is a part of normal care, comprehensive nutrition assessment have not been incorporated, and this impairs the ability to provide effective, critical nutrition intervention. ${ }^{9}$ To our knowledge, this is the first study to track sequelae while concurrently completing a nutrition assessment. Nutritional and weight status in PwPD varies over the course of the disease. ${ }^{8}$ These fluctuations can occur due to decline in cognitive and physical functioning. ${ }^{8}$ Fluctuations in weight and nutrition status can further compromise body composition, biochemical and clinical levels, cognitive and physical functioning, ${ }^{7,10,11}$ quality of life (QOL), and are associated with longer 
hospital stays. ${ }^{12,13}$ The purpose of this longitudinal, observational study was to track the nutritional status of PwPD over four years. A secondary aim of this study was to describe changes in PD sequelae, as these sequelae may help explain the possible changes in nutritional status that occur. It was hypothesized that change in motor and non-motor sequelae overtime can influence nutrition status and dietary intake, which in turn can impact health outcomes measured by biochemical, anthropometric and clinical data (Figure 1).

\section{Methods}

This was an ancillary study of a five-year observational, longitudinal study assessing the nutritional, cardio-metabolic, cognitive and physical function status of PwPD and acquired brain injury (ABI) (Longitudinal Study of Communication, Nutrition and Physical Activity). To be eligible for the original study, persons recruited via flyers, word-of-mouth, and announcements in support groups, had to be between 18-85 years of age and one-year post-PD or -ABI diagnosis; for this study, we only used data from PwPD. Participants completed assessment visits ( $\sim 3$ hours each) at the University of Rhode Island's Speech and Hearing Clinic every six months for five years.

Specifically, for this study, PwPD's were assessed at baseline, years two and four. The University's Institutional Review Board approved this study (IRB HU1314-006) and subjects provided written consent in accordance to the Declaration of Helsinki before enrolling. 


\section{Assessments.}

Nutrition assessment includes the examination of anthropometric measures, biochemical markers, clinical data, and dietary intake. ${ }^{14,15}$ Comprehensive nutrition assessments examined change in nutrition status overtime. This included assessments of diet quality, biochemical, anthropometric, and clinical data. To assess diet quality, participants completed the dietary screening tool (DST), a 25 -item questionnaire validated and used to identify dietary patterns and nutritional risk in older adults. ${ }^{16} \mathrm{~A}$ total of 105 points can be achieved and scores can be categorized as: at risk $(<60)$, possible risk (60-75), and not at nutrition risk $(>75)$. Serum total cholesterol, low-density lipoprotein cholesterol (LDL-C), high-density lipoprotein cholesterol (HDL-C), triacylglycerol, and glucose were obtained using a finger stick (Cholestech ${ }^{\circledR}$ LDX system, Hayward, CA) after a 12-hour fast. Blood pressure was measured using an automatic blood pressure machine. Height and weight were measured in duplicate and used to calculate body mass index (BMI, kg of body weight/height in meters ${ }^{2}$ ). The following criteria were used to characterize suboptimal assessment markers: 1) BMI >28 $\mathrm{kg} / \mathrm{m}^{2} ;{ }^{17}$ 2) systolic $>130 \mathrm{mmHg}$ and diastolic blood pressure $>80 \mathrm{mmHg} ; 3$ ) total cholesterol (TC) $>200 \mathrm{mg} / \mathrm{dL}$; 4) high-density lipoprotein cholesterol (HDLC) $<40 \mathrm{mg} / \mathrm{dL}$; 5) low-density lipoprotein cholesterol (LDL-C) $>100 \mathrm{mg} / \mathrm{dL} ; 6)$ triacylglycerol (TAG) $>150 \mathrm{mg} / \mathrm{dL} ; 7$ ) fasting glucose $\geq 100 \mathrm{mg} / \mathrm{dL}$.

The following assessments were used to describe change in disease sequelae. The short physical performance battery (SPPB) ${ }^{18}$ assessed 
physical functioning. The Repeatable Battery for the Assessment of Neuropsychological Status (RBANS) ${ }^{19}$ assessed cognitive function; scores $<80$ were indicative of cognitive impairment. Both the Swallowing Quality of Life (SWAL-QOL) Survey ${ }^{20}$ and a timed swallow test $(\mathrm{ml} / \mathrm{s}){ }^{21}$ assessed swallow function. A SWAL-QOL score of $<75 \%$ and a time swallow speed of $<10 \mathrm{ml} / \mathrm{s}$ was considered suboptimal. Finally, a medical history questionnaire is used to identify health-related conditions and changes in health conditions at each assessment visit.

Statistical Analysis. Data were analyzed in SPSSv26. Categorical variables are represented as numbers and percentages and continuous variables are reported as mean \pm standard deviations. Data were assessed for normality and non-normally distributed data were transformed (square root or reflect and square root) for analyses, mean \pm standard deviations of variation before transformation are reported. A repeated measures analysis of covariances was used to examine change overtime among outcome variables. Time since diagnosis was used as a covariate. A Bonferroni adjustment was made for multiple comparisons. Analyses were 2 -tailed and a $p<0.05$ indicates statistical significance. Participants were then individually analyzed for suboptimal scores across each outcome variable and the frequency of suboptimal scores were reported.

\section{Results}

Eight PwPD were analyzed over four years. At baseline, the mean age was $67.1 \pm 4.0$ years and ranged from $62-72$ years. The average time since 
diagnosis was $8.1 \pm 7.5$ years, ranging from $1-23$ years. Six out of eight participants were male (75\%). All participants identified as Caucasian. One participant reported attending some college, another had a college degree, and six achieved a post-baccalaureate degree. At baseline, two participants identified as smokers but quit during the first two years of the study. Three participants had deep brain stimulation, and one participant had the diagnosis of PD with Lewy Body Dementia. Two PwPD had thyroid conditions, and one PwPD had a history of a myocardial infarction.

Nutrition Assessment Markers. There was no significant change in nutrition assessment markers overtime (Table 1). At baseline and year 2 , seven participants were at possible- or at nutrition- risk, while six participants were at possible- or at- nutrition risk at year 4. Three PwPD had BMI scores $>28 \mathrm{~kg} / \mathrm{m}^{2}$ at baseline, which decreased to two PwPD at years 2 and 4 . For one PwPD height and weight could not be measured at year 4 due to functional decline. Over the four years, four participants experienced a ten pound or greater weight loss. One PwPD remained within one pound of their baseline body weight, and one PwPD experienced a six-pound weight gain.

The number of PwPD with elevated systolic blood pressure (SBP) increased from four to seven overtime, but the number of PwPD with elevated diastolic blood pressure (DBP) decreased from five to two overtime. Only one participant had elevated total cholesterol at baseline, two participants had elevated total cholesterol at year 2 and one participant had elevated total cholesterol at year 4. At baseline three participants had elevated LDL-C, while 
four PwPD had elevated LDL-C at year 2 and three at year 4. Four participants presented with low HDL-C at year 1, which decreased to two participants at years 2 and 4 . At baseline, three PwPD had abnormal fasting TAG levels, while one PwPD at year 2 and two PwPD at year 4 had elevated fasting TAG levels. One PwPD had elevated fasting glucose levels at baseline and year 2, however four participants had elevated levels at year 4 . All participants had at least one suboptimal lab value or blood pressure reading at each visit.

Disease Sequelae. Outcome variables used to describe disease sequelae at each time point are summarized in Table 2. At baseline all participants had RBANS scores indicative of normal cognitive functioning. At year $2(37.5 \%, n=3)$ had RBANS scores $<80$ and $50 \%(n=4)$ had RBANS scores $<80$ at year 4 . Three PwPD (37.5\%) had SPPB scores $<10$ at baseline, while four $(50 \%)$ had SPPB scores $<10$ at years 2 and 4 . At baseline two PwPD had SWALQOL scores $<75 \%$, while four participants had suboptimal scores at year 2, and two PwPD at year 4. Two participants had suboptimal timed swallow speeds ( $<10$ seconds) at baseline, and four participants had suboptimal timed swallow speeds at years 2 and 4 .

\section{Discussion}

Findings indicate most PwPD were at nutrition risk overtime, experienced weight loss and presented with multiple cardiovascular risk factors. While there was no significant mean change in outcome variables related to cognition, physical functioning or swallowing, many participants exhibited decline in these areas over time. 
Nutrition Related Outcome Variables. Throughout this study most PwPD were at possible or at nutrition risk. Previous research has found up to $63 \%$ of PwPD to be categorized as with malnutrition or at risk for malnutrition ${ }^{22}$, however there is limited research regarding the overall diet quality of PwPD. The current study adds to previous research regarding weight status among PwPD. Previous research has found over $50 \%$ of PwPD to experience weight loss but present with elevated waist circumference. ${ }^{8,22}$

This study also adds to the body of research on biochemical and clinical assessment markers that may help not only assess nutritional status, but also cardiometabolic status. Our sample presented with multiple cardiometabolic risk factors throughout the course of the study despite the modest decline in weight. Overtime there was an increase in HDL-C levels among PwPD, while not significant, there was large effect size. Additionally, the number of PwPD with low HDL-C levels decreased by year 4 . Previous research suggests a cardiometabolic protective effect of PD and theorizes that optimal HDL-C levels among PwPD may explain this theory. ${ }^{23}$ While improvements in HDL-C levels were observed, the number of participants with elevated fasting glucose and triglycerides increased. This may be attributed to the change in taste PwPD experience, as an affinity for sugar and sweets is common ${ }^{8}$, which can impact glucose and triglyceride levels.

Disease Sequelae Outcome Variables. The increase in the number of PwPD with suboptimal cognition, physical functioning, and swallowing is consistent with the literature. ${ }^{24}$ At year 4, 50\% of PwPD in this study had 
suboptimal assessment markers related to cognitive, physical, and swallow function. Past research has found a relationship between physical activity level and/or physical functioning with cognition and well-being measures among PwPD. ${ }^{25,26}$ The decline in swallow function among participants may help explain the presence of weight loss and consistently poor diet quality among PwPD. Previous research has found PwPD with dysphagia are more likely to experience unintentional weight loss and to avoid foods that can be part of a nutritious diet, such as fruits and vegetables. ${ }^{10}$ Current findings, along with this previous research, support the need for an interdisciplinary treatment approach for managing PD to promote health-related QOL.

Study Strengths and Limitations. This is the first study to evaluate the change in nutritional status among a cohort of PwPD over four years in conjunction with change in motor and non-motor sequelae. This study provides subjective and objective data that provides a holistic picture of how the nutrition and health status of PwPD can change overtime. Finally, the interdisciplinary nature of this study can help inform future screenings and the care management of PwPD. Study results can be incorporated into the World Health Organization International Classification of Functioning, Disability, and Health (ICF) model has been used a framework to assess PwPD's health, health related function and QOL. ${ }^{25}$ The ICF model consists of five domains that cover all aspects related to a person's health status and human function and include: 1) body functions and structures, 2) activities, 3) mental health factors, 4) environmental factors, and 5) personal factors. ${ }^{25}$ Future should can 
expand upon this interdisciplinary assessment of PwPD to also screen for mental health factors.

While this study is novel, it is not without limitations. Our small sample size warrants tracking of a larger cohort of PwPD overtime. To see significance differences in DST scores, a total of 28 participants would have been needed to achieve a power of 0.80 and a large effect size $\left(\eta_{p}{ }^{2}=0.25\right)$. Based on our sample size of eight participants a power of 0.05 was achieved. The generalizability of study findings is limited by the fact that the majority of our participants were highly educated, white, male, and recruited from the University's Speech and Hearing Clinic. However, epidemiological studies have reported the majority of American PwPD are white, and this could be due racial and socioeconomic disparities in receiving treatment for PD. ${ }^{27}$ Additionally, one participant's physical functioning had declined so much from baseline that a height and weight was not obtained at year 4. However, inability to complete certain assessments sheds light onto the progression and realities of PD. Future research should track a larger, more diverse sample of PwPD to better understand how nutritional status changes throughout the course of PD and explore if a synergetic relationship exists between nutrition, cognition, physical functioning, and disease progression.

\section{Conclusion}

This study adds to the body of literature finding PwPD to have poor nutritional status and diet quality. ${ }^{9,28}$ Participants in this study experienced weight loss and changes in biochemical and clinical values, which supports 
the need for routine, interdisciplinary assessment for PwPD to identify and treat disease sequelae and promote health-related QOL. Future research should explore the efficacy of interdisciplinary interventions. This interdisciplinary team should include nutrition professionals, such as registered dietitians. Additionally, due to the observed decline in cognitive and physical functioning among PwPD research should also consider including informal caregivers whose role around buying, preparing, and serving nutritious foods may increase as PD advances.

\section{Chapter 1 Table and Figures}

Figure 1: Propose Mechanism of How Disease Sequalae Impact Nutrition Status Which May Further Impact Health Outcome

Motor and Non-
Motor Sequelae $\Rightarrow \begin{gathered}\text { Nutritional } \\ \text { Status and Diet } \\ \text { Intake }\end{gathered} \Rightarrow \begin{gathered}\text { Biochemical, } \\ \text { Anthrpometric, } \\ \text { Clinical Values }\end{gathered}$


Table 1: Nutrition Assessment Markers Among PwPD Over 4 Years

\begin{tabular}{|c|c|c|c|c|c|}
\hline & Baseline & Year 2 & Year 4 & $p$ & $\eta_{p}^{2}$ \\
\hline DST & $64.0 \pm 13.8$ & $66.4 \pm 8.8$ & $64.3 \pm 13.2$ & 0.8 & 0.04 \\
\hline Weight (lbs.) ${ }^{a}$ & $176.3 \pm 29.7$ & $169.0 \pm 29.2$ & $169.0 \pm 25.5$ & 0.9 & 0.01 \\
\hline BMI $\left(\mathrm{kg} / \mathrm{m}^{2}\right)^{\mathrm{a}}$ & $27.6 \pm 3.4$ & $26.6 \pm 3.1$ & $26.6 \pm 2.2$ & 0.9 & 0.02 \\
\hline SBP (>130 mmHg) & $141.4 \pm 22.4$ & $140.3 \pm 17.2$ & $144.1 \pm 14.5$ & 0.06 & 0.38 \\
\hline DBP (>80 mmHg) & $81.1 \pm 10.4$ & $78.8 \pm 14.2$ & $80.1 \pm 12.4$ & 0.9 & 0.46 \\
\hline $\mathrm{TC}(>200 \mathrm{mg} / \mathrm{dL})$ & $175.1 \pm 25.2$ & $165.1 \pm 33.2$ & $163.9 \pm 22.0$ & 0.6 & 0.07 \\
\hline LDL-C (100mg/dL) & $104.0 \pm 22.6$ & $93.75 \pm 30.9$ & $87.4 \pm 36.2$ & 0.99 & 0.0 \\
\hline HDL-C (<40mg/dL) & $44.1 \pm 17.4$ & $49.0 \pm 17.7$ & $48.1 \pm 12.4$ & 0.53 & 0.1 \\
\hline TAG (>150 mg/dL) & $134.9 \pm 68.3$ & $112.9 \pm 45.7$ & $143.0 \pm 80.4$ & 0.88 & 0.02 \\
\hline GLU (>100mg/dL) ${ }^{a}$ & $89.0 \pm 9.4$ & $89.2 \pm 7.2$ & $95.0 \pm 12.2$ & 0.66 & 0.07 \\
\hline Statin Medication (n(\%)) & $6(75)$ & $6(75)$ & $6(75)$ & NA & NA \\
\hline BP Medication (n(\%)) & $4(50)$ & $4(50)$ & $3(37.5)$ & NA & NA \\
\hline
\end{tabular}

${ }^{a} n=7$; effect size $0.01=$ small, $0.06=$ moderate; $0.14=$ large. Abbreviations: $D S T=d i e t a r y$ screening tool, $\mathrm{BMI}=$ body mass index, $\mathrm{SBP}=$ systolic blood pressure, $\mathrm{DBP}=$ diastolic blood pressure, $\mathrm{TC}=$ total cholesterol, $\mathrm{LDL}-\mathrm{C}=$ =low-density lipoprotein cholesterol, $\mathrm{HDL}-\mathrm{C}=$ high density lipoprotein cholesterol, TAG=triacylglycerol, $\mathrm{GLU}=$ glucose, $\mathrm{BP}=$ blood pressure

Table 2: Change in Disease Sequelae (Cognition, Physical Function, Swallowing) Among PwPD Over Four Years

\begin{tabular}{|l|c|c|c|c|c|}
\hline & Baseline & Year 2 & Year 4 & $\boldsymbol{p}$ & $\eta_{\mathrm{p}}{ }^{2}$ \\
\hline RBANS & $93.0 \pm 13.3$ & $88.6 \pm 22.4$ & $81.6 \pm 24.2$ & 0.4 & 0.1 \\
\hline SPPB & $9.9 \pm 1.8$ & $8.8 \pm 2.1$ & $8.4 \pm 3.3$ & 0.8 & 0.03 \\
\hline SWALQOL & $77.3 \pm 12.4$ & $72.5 \pm 12.2$ & $75.6 \pm 16.1$ & 0.3 & 0.2 \\
\hline Swallow Speed (ml/secs) & $15.7 \pm 9.8$ & $15.01 \pm 11.1$ & $13.5 \pm 10.6$ & 0.8 & 0.03 \\
\hline
\end{tabular}

${ }^{a} n=7 ; \eta_{p}{ }^{2}$ values can be interpreted as: $0.01=$ small effect, $0.06=$ moderate effect, $0.14=$ large effect. Abbreviations: RBANS=Repeatable Battery for the Assessment of Neuropsychological Status; SPPB=short physical performance battery; SWALQOL=swallowing quality of life

\section{References}

1. Fahn S. Description of Parkinson's disease as a clinical syndrome. Annals of the New York Academy of Sciences. 2003;991:1-14.

2. O'Brien JA, Ward A, Michels SL, Tzivelekis S, Brandt NJ. Economic burden associated with Parkinson disease. Drug Benefit Trends. 2009;21(6):179-190. 
3. Borlongan CV, Burns J, Tajiri N, et al. Epidemiological survey-based formulae to approximate incidence and prevalence of neurological disorders in the United States: A meta-analysis. PloS one. 2013;8(10):e78490.

4. Cilia R, Cereda E, Klersy C, et al. Parkinson's disease beyond 20 years. J Neurol Neurosurg Psychiatry. 2014;86(8):849-855.

5. Reedy J, Lerman JL, Krebs-Smith SM, et al. Evaluation of the Healthy Eating Index-2015. Journal of the Academy of Nutrition and Dietetics. 2018;118(9):1622-1633.

6. Nutt JG, Wooten GF. Clinical practice. Diagnosis and initial management of Parkinson's disease. The New England Journal of Medicine. 2005;353(10):1021-1027.

7. Vikdahl M, Domellof ME, Forsgren L, Haglin L. Olfactory function, eating ability, and visceral obesity associated with MMSE three years after Parkinson's disease diagnosis. The journal of Nutrition, Health \& Aging. 2015;19(9):894-900.

8. Barichella M, Cereda E, Pezzoli G. Major nutritional issues in the management of Parkinson's disease. Movement Disorders. 2009;24(13):1881-1892.

9. LoBuono DL, Taetzsch AG, Lofgren IE, Xu F, Delmonico MJ, Mahler L. Cognitive status and cardio-metabolic risk of patients with acquired brain injury and Parkinson's disease. Disability and Health Journal. 2015;9(1):134-139.

10. Lorefalt B, Ganowiak W, Palhagen S, Toss G, Unosson M, Granerus AK. Factors of importance for weight loss in elderly patients with Parkinson's disease. Acta Neurologica Scandinavica. 2004;110(3):180187.

11. Wills AA, Perez A, Wang J, et al. Association between change in body mass index, Unified Parkinson's Disease Rating Scale Scores, and survival among persons with Parkinson disease: Secondary analysis of 
longitudinal data From NINDS exploratory trials in Parkinson disease long-term study 1. JAMA Neurology. 2016;73(3):321-328.

12. Sheard JM, Ash S, Silburn PA, Kerr GK. Prevalence of malnutrition in Parkinson's disease: a systematic review. Nutrition Reviews. 2011;69(9):520-532.

13. Sheard JM, Ash S, Mellick GD, Silburn PA, Kerr GK. Markers of disease severity are associated with malnutrition in Parkinson's disease. PloS one. 2013;8(3):e57986.

14. Frisancho AR. New standards of weight and body composition by frame size and height for assessment of nutritional status of adults and the elderly. Am J Clin Nutr. 1984;40(4):808-819.

15. White JV, Guenter P, Jensen G, et al. Consensus statement of the Academy of Nutrition and Dietetics/American Society for Parenteral and Enteral Nutrition: characteristics recommended for the identification and documentation of adult malnutrition (undernutrition). Journal of the Academy of Nutrition and Dietetics. 2012;112(5):730-738.

16. Bailey RL, Mitchell DC, Miller CK, et al. A dietary screening questionnaire identifies dietary patterns in older adults. J Nutr. 2007; 137:421-426.

17. Winter JE, Maclnnis RJ, Wattanapenpaiboon N, Nowson CA. BMI and all-cause mortality in older adults: a meta-analysis. Am J Clin Nutr. 2014;99(4):875-890.

18. Guralnik JM, Simonsick EM, Ferrucci L, et al. A short physical performance battery assessing lower extremity function: association with self-reported disability and prediction of mortality and nursing home admission. Journal of Gerontology. 1994;49(2):M85-94.

19. Randolph C. Repeatable Battery for Assessment of Neuropyschological Status. In. Examiner's Manual San Antonio, TX: The Psychological Corporation 1998. 
20. McHorney CA, Robbins J, Lomax K, et al. The SWAL-QOL and SWALCARE outcomes tool for oropharyngeal dysphagia in adults: III.

Documentation of reliability and validity. Dysphagia. 2002;17(2):97-114.

21. Nathadwarawala KM, Nicklin J, Wiles CM. A timed test of swallowing capacity for neurological patients. J Neurol Neurosurg Psychiatry. 1992;55(9):822-825.

22. Carmo TPS, Ferraz AC. Nutritional assessment and the use of levodopa with protein meals among patients with Parkinson's disease in the city of Macaé, Rio de Janeiro, Brazil. Conference Proceedings 2016.

23. Cassani E, Cereda E, Barichella M, et al. Cardiometabolic factors and disease duration in patients with Parkinson's disease. Nutrition. 2013;29(11-12):1331-1335.

24. Kalia LV, Lang AE. Parkinson's disease. Lancet. 2015;386(9996):896912.

25. van Uem JMT, Cerff B, Kampmeyer M, et al. The association between objectively measured physical activity, depression, cognition, and health-related quality of life in Parkinson's disease. Parkinsonism \& Related Disorders. 2018;48:74-81.

26. Stegemoller EL, Nocera J, Malaty I, et al. Timed up and go, cognitive, and quality-of-life correlates in Parkinson's disease. Archives of Physical Medicine and Rehabilitation. 2014;95(4):649-655.

27. Hemming JP, Gruber-Baldini AL, Anderson KE, et al. Racial and socioeconomic disparities in parkinsonism. Archives of Neurology. 2011;68(4):498-503.

28. Sheard JM, Ash S, Mellick GD, Silburn PA, Kerr GK. Malnutrition in a sample of community-dwelling people with Parkinson's disease. PloS one. 2013;8(1):e53290. 


\section{CHAPTER TWO}

Diet Quality and Nutrition Concerns of People

with Parkinson's Disease and Their Informal Caregivers: A Mixed Methods Study

By

Dara L. LoBuono ${ }^{1}$, Kyla S. Shea ${ }^{2}$, Alison Tovar ${ }^{3}$, Skye N. Leedahl' ${ }^{4}$, Leslie Mahler $^{5}$, Furong $\mathrm{Xu}^{6}$, Ingrid Lofgren ${ }^{7}$

To be submitted to: The Journal of the Academy of Nutrition and Dietetics

1

PhD Candidate, Department of Nutrition and Food Sciences, The University of Rhode Island, Kingston, RI 02881. Email: dara lobuono@uri.edu

2

Undergraduate Research Assistant, Department of Nutrition and Food Sciences, The University of Rhode Island, Kingston, RI 02881. Email: kyla shea@my.uri.edu

${ }^{3}$ Associate Professor, Department of Nutrition and Food Sciences, The University of Rhode Island, Kingston, RI 02881. Email: alison tovar@uri.edu

${ }^{4}$ Associate Professor, Department of Human Development and Family Studies, The University of Rhode Island, RI, 02881. Email: skyeleedahl@uri.edu

${ }^{5}$ Associate Professor, Department of Communicative Disorders, The University of Rhode Island, RI 02811. Email: Imahler@uri.edu

${ }^{6}$ Professor, Department of Kinesiology, The University of Rhode Island, RI 02881. fxu2007@uri.edu

${ }^{7}$ Professor, Department of Nutrition and Food Sciences, The University of Rhode Island, RI, 02881. Email: ingrid lofgren@uri.edu 
Diet Quality and Nutrition Concerns of People with Parkinson's Disease and Their Informal Caregivers: A Mixed Methods Study

By

Dara L. LoBuono ${ }^{1}$, Kyla S. Shea ${ }^{1}$, Alison Tovar ${ }^{1}$, Skye N. Leedahl ${ }^{2}$, Leslie Mahler $^{3}$, Furong $\mathrm{Xu}^{4}$, Ingrid E. Lofgren ${ }^{1}$

${ }^{1}$ Department of Nutrition and Food Sciences, ${ }^{2}$ Departmetn of Human Development and Family Studies, ${ }^{3}$ Department of Communicative Disorders, ${ }^{4}$ Department of Kinesiology, The University of Rhode Island, Kingston RI

\section{Abstract}

Background: People with Parkinson's disease (PwPD) are often at nutritional risk. Limited research exists regarding the diet quality or nutritional concerns of PwPD and informal caregivers. Using patient-caregiver dyads is an innovative model to assess nutrition and understand dietary needs.

Objective: Data collected from dyads were used to evaluate the diet quality and describe self-reported nutrition concerns of PwPD and their caregivers. Whether or not there was a consistent pattern between nutrition concerns and diet quality among PwPD and caregivers was also explored.

Methodology: A mixed-methods study design assessed 20 PwPD-caregiver dyads. During home visits, semi-structured, dyadic interviews were audiorecorded for qualitative data and anthropometrics and questionnaires were collected. Two phone 24-hour recalls were completed to collect dietary intake. Dietary data was assessed for diet quality using the Healthy Eating Index (HEI)-2015 scoring metric. 
Statistical Analyses Performed: Diet quality descriptives for PwPD and caregivers were reported as mean \pm standard deviation. Qualitative data was analyzed in NVivo and inter-coder reliability was $>90 \%$. Qualitative data was charted into framework matrices and reported as frequencies to quantify codes. A side-by-side comparison of themes and HEI-2015 scores for each participant was conducted.

Results: Mean participant age was $68.1 \pm 11.2$ years. Mean HEI-2015 scores for PwPD was $58.3 \pm 12.4$ and $58.1 \pm 10.6$ for caregivers, translating to an $F$ letter grade. Dietary concerns related to PD sequelae included: change in appetite or amount eaten, gastrointestinal issues, food-medication management, chewing/swallowing issues, and change in taste/smell. A large amount of variation between $\mathrm{HEI}-2015$ scores and self-reported nutrition concerns were detected.

Conclusions: Poor diet quality may be attributed to self-reported nutrition concerns. Presence of poor diet quality and nutrition concerns among dyads suggests including both nutrition professionals and caregivers to promote nutritional health among PwPD. Future research should examine the number of nutrition concerns to help identify readiness to make dietary changes among dyads.

Keywords (5 key words minimum): Parkinson's disease, caregivers, nutrition, dietary intake, mixed methods, diet, food and nutrition 


\section{Introduction}

Parkinson's disease (PD) is a progressive neurodegenerative movement disorder that impacts nearly one million Americans. ${ }^{1,2}$ Disease stage and sequalae (conditions that result specifically from PD), physiological factors and treatments and associated side effects of PD can compromise dietary intake and quality. ${ }^{3,4}$ Disease sequelae impact motor (slowness of movement, shuffling/freezing gait, muscle rigidity ${ }^{5,6}$ ) and non-motor (fatigue, cognitive changes, difficulty swallowing, change in taste and smell, gastrointestinal issues $^{3,7-9}$ ) function, which can worsen overtime and impact dietary intake, nutritional status, and body composition ${ }^{3}$ Suboptimal weight status and body composition can further impact physical function and cognition ${ }^{10-12}$, quality of life (QOL), and health outcomes..$^{13}$ Consequently, informal caregiver responsibilities increase as PD progresses,${ }^{14}$ and caregivers are typically responsible for performing the majority of activities of daily living (ADL) for people with PD (PwPD), ${ }^{15}$ including assisting with or doing the buying, preparing, and consuming meals and snacks. ${ }^{16,17}$

Nutrition is integral in managing PD. ${ }^{3}$ However, there is limited research related to diet quality among PwPD and caregivers, populations who are at risk for poor dietary quality. To help improve dietary patterns in at-risk populations, understanding food choices and exploring acceptable sources of nutrition advice and support is essential. ${ }^{18}$ The Healthy Eating Index (HEI)2015 is a diet quality index that assesses the compliance with the Dietary Guidelines for Americans (DGAs). ${ }^{19}$ The DGAs are evidenced-based 
recommendations informed by diet quality's impact on health outcomes, such as weight status, diabetes, cancer and cardiovascular disease..$^{19,20}$

Adhering to the DGAs could help PwPD meet the requirements for what are several nutrients of concern, such as fiber ${ }^{21}$, fluid ${ }^{21}$, vitamin $D^{22}$, vitamin $\mathrm{E}^{23}$, omega-3 fatty acids ${ }^{23}$, and protein ${ }^{22}$. These nutrients can be obtained through an adequate diet that aligns with the dietary guidelines. However, research has found that PwPD have poor diet quality inconsistent with dietary patterns that promote health, such as the DGA or the Mediterranean Diet Pattern. ${ }^{24,25}$ Findings warrants the exploration of differences in overall dietary patterns between PwPD and their informal caregivers. ${ }^{26}$

It is particularly important to assess the diet quality of caregivers, as caregiver stress and burden can adversely affect caregiver's psychosocial and physical functioning, which can compromise care provided and the health of the patient-caregiver dyad ${ }^{27,28}$. Research has examined the nutrient intake of PwPD and their spouses ${ }^{26}$, but has not specifically analyzed the diet quality in the context of the patient-caregiver dyads. Additionally, exploration of nutrition concerns of these dyads and how these concerns compare to diet quality have not been explored. Compared to the general population, caregivers for PwPD are more likely to have depression, anxiety, decreased health status ${ }^{27}$, and/or poorer $\mathrm{QOL}^{29}$, which may impact diet quality. Additionally, given the later onset of PD, most caregivers are older and have their own medical conditions that may require dietary modifications. ${ }^{30}$ 
Qualitative work is needed to explore PwPD's and their caregivers' views on dietary intake and decisions that impact that intake. Such nutrition research has been used in similar populations, like older adults and their informal caregivers, to better understand factors impacting dietary choices and inform appropriate and acceptable services. ${ }^{31,32}$ This qualitative work has also been conducted to better understand various aspects of care among PwPD and their caregivers, such as coping with the disease ${ }^{33,34}$, managing cognitive changes ${ }^{35}$, and QOL. ${ }^{36}$ However, such an approach has not been used to understand the nutrition concerns of this population.

Understanding the diet quality and the nutrition concerns of both PwPD and their caregivers can inform nutrition services for managing PD, and how best to facilitate dietary management from patient perspective. Additionally, gathering this information can help identify general services required to facilitate the process of learning to live with PD. ${ }^{37}$ As a result, to effectively design a nutrition intervention for PwPD and caregivers, assessing dietary quality as well as collecting qualitative information related to their nutrition concerns is vital to develop an effective and sustainable nutrition service. The main purposes of this study were to: 1) evaluate the diet quality among PwPD and their informal caregivers; 2 ) describe the self-reported nutrition concerns among PwPD and their informal caregivers; and 3) explore if there was a consistent pattern between self-reported nutrition concerns and dietary quality of PwPD and informal caregivers. 


\section{Methods}

A concurrent mixed-method design ${ }^{38}$ was used to assess diet quality and nutrition concerns of PwPD and their informal caregivers. This mixed-methods design was chosen to gain a more complete understanding of nutritional needs of PwPD and caregivers through comparing and synthesizing both quantitative and qualitative data. ${ }^{38}$ Data was collected over four assessments completed between November 2018 and April 2019. Participants were recruited from support groups throughout New England, New York, and New Jersey via announcements and flyers at community centers, via healthcare providers, and through popular press coverage from the University.

Assessment 1 was an informational phone call during which participants were screened for eligibility and informed about the study protocol. Both the PwPD and their informal caregiver were required to participate and needed to be community-dwelling, English-speaking, and $\geq 18$ years old. Participants also needed to score $\geq 18$ on the Telephone Montreal Cognitive Assessment ( $T$ MoCA), a cognitive screening tool. ${ }^{39,40}$ At the beginning of Assessment 2, which was an in-person visit, the study protocol was reviewed and both PwPD and their informal caregiver completed the informed consent process. Participants completed a timed-swallow test, questionnaires, and a semistructured, dyadic interview. Assessments 3 and 4 were phone calls during which participants completed two 24 -hour recalls. Findings from this study were a part of a larger study seeking to inform features of a digital health nutrition intervention. Approval from the University of Rhode Island's 
Institutional Review Board (HU1819-001) was obtained.

\section{Data Collection}

Nutrition assessment data are highlighted in Table 2. The two 24-hour recalls included one week and one weekend day, and were conducted using the gold-standard, multiple-pass interview method. ${ }^{41}$ Participants received a food amounts booklet to help estimate and report accurate portion sizes. The 24-hour recalls were entered into Nutrition Data System for Research Software 2017 (NDSR, University of Minnesota, Minneapolis, MN) and analyzed for total energy and nutrient intake. The NDSR data were assessed for diet quality using the HEI-2015 scoring metric. ${ }^{42}$ Outputs from NDSR were used to calculate HEI-2015 component scores derived using SAS codes. ${ }^{42}$ Total HEI-2015 scores are based on 1-100; the higher the score, the better the diet quality. A graded approach was used to categorize HEI-2015 scores $(A=90-100, B=80-89, C=70-79, D=60-69$, and $F=0-59) .{ }^{42}$

During Assessment 2, height was measured using a stadiometer (Seca, Birmingham, UK) to the nearest $0.5 \mathrm{~cm}$ in duplicate and then averaged and weight was obtained using a calibrated scale (Tanita HD351 digital scale, Arlington Heights, IL) to the closest $0.1 \mathrm{~kg}$ and in duplicate and then averaged. These values were used to calculate body mass index (BMI, kg of body weight/height in meters ${ }^{2}$ ). A foot-to-foot bioelectrical impedance analysis device (Tanita BF-556, Arlington Heights, IL), estimated body fat percentage. ${ }^{43}$ Body fat was not obtained for participants with implanted medical devices. Waist circumference was measured at the top of the hip bone using Gulick 
anthropometric tape (Fabrication Enterprises Inc. White Plains, NY) in duplicate and averaged. ${ }^{44}$ Finally, participants also completed a medical history and demographic information (age, gender, race/ethnicity, education level, employment status) survey.

Semi-structured, dyadic interviewing was used to capture information related to dietary intake and nutrition concerns using a pre-prepared moderator guide (Appendix A). The research team developed the moderator guide with reference to previous literature..$^{3,37,45}$ Twenty-four opened- and closed-ended questions were used that fit into one of three main domains: PD and Diet, Accessibility of Nutrition and Health Information, and Digital Health for PD. Semi-structured dyadic interviews also contained three closed-ended questions which provided insight into PwPD's and caregivers' perceptions of healthy eating and its usefulness for managing disease. Interviews were conducted in the participants' homes by a doctoral candidate who was also a registered dietitian (DL) and audio recorded using a digital recorder. The mean length of interviews were approximately 39 minutes and interviews lasted from 21 to 64 minutes in length.

\section{Data Analyses}

Quantitative data analyses. Quantitative data was analyzed using SPSS v26 (IBM Corp, Summers, NY). Descriptive statistics are reported as mean \pm standard deviation for continuous variables and percentages and frequencies for categorical variables. Outliers were identified using boxplots (points that extend more than 1.5-box lengths from the edge of the box) 
among the following HEI-2015 variables: HEI-2015 Total Scores, Protein, and Refined Grains component scores. To explore differences between PwPD and caregivers, independent samples t-tests were used for all normally distributed continuous variables and Mann-Whitney $U$ tests examined differences between non-normally distributed continuous variables. Finally, the percent and frequency of PwPD and caregivers who met $>80 \%$ of adequacy and moderation HEl-2015 components scores were calculated. A p-value of $<0.05$ indicated statistical significance.

Qualitative Data Analyses. Qualitative data was analyzed using the framework analysis method ${ }^{46}$ and Colaizzi's Strategy in Descriptive Phenomenology. ${ }^{47}$ The following steps were taken to analyze transcripts. Recordings were transcribed verbatim and DL checked transcripts for accuracy. Transcripts were analyzed by $\mathrm{DL}$ and a trained research assistant (KS). The analyses focused on five questions from the moderator guide related to diet and nutrition.

Before coding individually, KS and DL read through all of the transcripts and developed a list of initial impressions and themes within three categories: Dietary Concerns Related to PD Sequelae, Other Nutrition Concerns, and Perceptions of Diet (Figure 1). Dietary concerns were coded deductively while themes that emerged from the transcripts were coded inductively and fell into the category of Other Nutrition Concerns or Perceptions of Diet. Both DL and KS coded one transcript from each batch independently and met to compare and reconcile coding. Then $\mathrm{DL}$ and $\mathrm{KS}$ created a working analytical 
framework and agreed upon codes to use for analyzing the remaining transcripts. The transcripts were uploaded to NVivo12 (QSR International Pty Ltd, AU) and coded separately by DL and KS. An inter-coder reliability was calculated, with an agreement $\geq 93 \%$ achieved. ${ }^{48}$

$\mathrm{DL}$ and KS compared codes and reconciled differences between codes, until a consensus was reached, and codes were finalized. To ensure information related to dietary intake was not overlooked in other sections of the transcripts, DL went through all of the transcripts and coded the remaining sections. KS reviewed the codes to verify coding structure, and added additional codes when needed, and DL and KS discussed differences in coding and collapsed themes. The larger research team and DL met to further collapse and finalize themes.

Data Integration. The research team reviewed both data sets and considered the qualitatively-coded themes in conjunction with the findings from the statistical analyses of the HEI-2015 scores. Qualitative data was charted into framework matrices and reported as frequencies using NVivo12 to quantify codes within the over-arching category of Dietary Concerns Related to PD Sequelae. This frequency data derived from the qualitative themes and a side-by-side comparison of themes were compared with individual HEl-2015 Scores for each participant. Data was interpreted and connections were explored between HEI-2015 scores and frequency of self-reported Dietary Concerns Related to PD sequelae from PwPD and their caregivers. 


\section{Results}

Twenty dyads were assessed. Participant characteristics are summarized in Table 1. All participants identified as Caucasian and one PwPD identified as Hispanic. Sixteen of the twenty dyads lived together. Ninety percent of PwPD were taking levodopa-containing medication, ranging from 37 times per day.

To help describe our study population and support the research aims, Table 2 highlights diet assessment data of PwPD and caregivers. Both PwPD and caregivers had near optimal percent body fat and waist circumference though BMI scores indicated both groups were overweight. Dyads were consuming slightly below the acceptable macronutrient distribution range (AMDR) for carbohydrates, slightly above the AMDR range for fat and within the AMDR range for protein. People with PD consumed $0.9 \pm 0.4 \mathrm{~g} / \mathrm{kg}$ body weight protein per day and caregivers consumed $0.9 \pm 0.2 \mathrm{~g} / \mathrm{kg}$ body weight per day. While dyads were below the dietary reference intake for most vitamins and minerals, the majority were taking supplements. There was no difference in nutrition assessment variables between PwPD and caregivers.

\section{Dietary Quality Among PwPD and Caregivers.}

Total HEI-2015 and component scores for PwPD and caregivers are summarized in Table 3. Low HEl-2015 scores indicates poor diet quality consistent with the national average. ${ }^{49}$ Fifty-five percent of PwPD and $65 \%$ of caregivers had total $\mathrm{HEl}-2015$ scores that translates to an F letter grade. ${ }^{42}$ Examination of HEl-2015 and component scores for PwPD and caregivers are 
summarized in Figure 2 and highlights the number of PwPD and caregivesr who achieved $\geq 80$ of maximum scores for total HEl-2015 scores and component scores.

\section{Qualitative Results: Self-Reported Nutrition Concerns}

Dietary Concerns Related to PD Sequelae. There were 182 phrases coded into the category Dietary Concerns for PD Sequelae (Table 4). The major themes related to PD Sequelae are highlighted in Figure 1. Phrases where dyads described how PD impacted mealtime and eating ability were coded as Mealtime Related Issues. The major areas discussed around these meal-time related issues included three-major sub-themes: Chewing and Swallowing; The Time it Takes to Cook or Consume a Meal; and Physical Sequelae. Phrases where participants reported cutting up their food smaller, modifying textures to consume foods, coughing during meals, or taking more effort to chew their food, were coded as Chewing and Swallowing.

- "He has been advised to cut things into small pieces often, and to drink fluids as you eat"-CG17

Many participants also described how physical changes related to PD impacted their ability to eat certain foods and mealtime. Phrases were coded as Physical Sequelae when dyads described how tremor and other physical changes impacted their ability to consume certain foods.

- "Primarily, the tremor affects my ability to get soup or things on a fork up to my mouth" - PD2

Another sub-theme within Mealtime Related Issues was the Time It Takes to Cook or Eat a Meal. Several participants described how the length of time to 
prepare a meal or consume a meal could diminish their appetite or impact meal enjoyment.

- "I'm done and the dishes are already done, but l'm waiting for his dish" - CG14

Phrases related to a Change in the Amount Eaten, Appetite, or Weight Status were coded when participants described an increase, decrease or no change in appetite, amount of foods consumed and/or change in weight status. Most of the phrases coded described a decrease in appetite. However, some participants did notice they were eating more than they used to.

- "My appetite is less than it used to be, there is no doubt about it."PD6

- "I've always been able to eat a lot and consume the calories very well. Parkinson's you just have to eat." - PD17

Only in four interviews did participants describe change in weight status. One participant reported an increase in weight due to the medications. Two participants reported that their weight decreased. Another reported that their weight stayed the same since being diagnosed.

- 'I'm kind of grateful you're losing weight in that when and if you fall, l'm gonna have to help you get back up, and the more you weigh, you know l'm better off if you don't weigh as much." - CG14 to PD14

Phrases where dyads described managing issues such as constipation, diarrhea, and nausea were coded as Gastrointestinal Issues; most of these phrases coded were constipation-related.

- "A little bit of constipation...I try to eat a lot of salad lately, try to compensate a little." - PD18

- "I think for myself sometimes I tend to overcorrect, so then it's you know, say my stool has been running loose, then I overcorrect, and then I'm in a constipation phase." - PD10 
Phrases where dyads discussed issues with spacing levodopa containing medication and high protein meals or where dyads described sideeffects of medication that impacted dietary intake were coded as Managing PD Treatment and Diet.

- "I found online that milk is one of the worst proteins to have with my medications, so I have been spacing out having my milk more than an hour from when I take my meds. I used to mix the MiraLAX with milk, but now I will mix it with Gatorade... I noticed that my medication lasts 45 minutes longer." - PD01

- "The first medication that I was on for Parkinson's increased my appetite and caused insomnia. And of course, if you are awake, you are going to pick on food items, so I wound up putting on some weight for about a year." - PD7

Phrases were coded as Taste and Smell when participants described how loss of taste and/or sense of smell impacted their food choices and ability to enjoy foods. Many reported a higher affinity for sweet tasting foods such as ice cream and desserts, while others reported trying to decrease their sugar intake.

- "I've come to love ice cream, I have it every single night almost, I didn't used to do that..." - PD16

- "I lost my sense of smell in 1992...way before my Parkinson's diagnosis, yes, but I think they relate loss of smell to Parkinson's. So, it took some of the enjoyment of food..." - PD05

Phrases where dyads described trying to increase their fluid intake or recognizing they needed more fluids in their diet were coded as Fluid and Thirst.

- "We're trying to increase the amount of water weight that I drink" PD13 about fluid intake and managing low blood pressure. 
Other Nutrition Concerns. Five themes emerged within the category of Other Nutrition Concerns (Figure 1). In total, 143 phrases were coded related to Other Nutrition Concerns. Phrases around Understanding Nutrition Claims were coded in 19 out of 20 interviews. Phrases were coded as Understanding Nutrition Claims when dyads expressed a desire to better understand healthy eating, nutrition claims, nutrition for overall health, or nutrition claims for managing PD.

- "As I spoke to you earlier, the difference between good cholesterol and bad cholesterol, so that l'm making better choices." - PD19

- "Probably for my dad in particular it would be if for Parkinson's, do they recommend a higher protein, lower fat, lower carb diet? Or do they recommend complex carbs with protein? Maybe he could eat a healthier diet than he does." - CG07

Within the theme Managing Life were three sub-themes: Managing Other Conditions with PD, Managing PD and Life, and Managing Diet and Life. Besides managing Parkinson's disease, many PwPD had other health conditions they were dealing with, including: musculoskeletal issues, weight management, endocrine issues, gastrointestinal issues, cardiovascular issues, and brain disorders.

- "Well one thing we've learned, because he does have a tendency to [get] gout, there's certain vegetables to avoid that will contribute to uric acid forming in the joints." - CG17

- "I sort of have breakfast, but don't really have lunch, and then at dinner I eat a lot...I think it's more my Concerta wears off, and then um, because it's considered an appetite suppressant, and then l'm just really hungry." - CG12 
Within the Managing Life theme was also the sub-theme, Managing PD and Life. Participants described challenges with overcoming life events and challenges and managing PD.

- "Well Parkinson's definitely makes you more emotional. l've always been an emotional person. I cried when we got married." - PD14

A few participants described day-to-day and life events that could impact their dietary intake, such phrases were coded as Managing Diet and Life. One participant felt dealing with personal matters impacted their dietary choices but felt PD did not.

- 'People who don't think you're just going through a fad or trying to be effected by something, when really you're just trying to eat healthy, especially for a medical condition, you know, 'oh well she's on one of those diets or she's being one of those people'" - PD10

- "I have been not watching what I eat over the past 18 months, but it does not have to do with Parkinson's, it has to do with stress... stress eating - not feeling good and eating the wrong foods." - PD20

Many participants described how they were currently involved with complimentary care services to help manage PD. Phrases where participants described partaking in exercise programs, acupuncture, dietary changes, or support groups were coded as Alternative Practices or Medicine.

- "There was a cleanse that was put out by Kripalu, the yoga center, it's like a 3 week cleanse to purge your body of various toxins, you know it's hard, but once you get into it its really good, you feel really healthy and vibrant"-PD11

- "My acupuncturist, who I respect and think is bright, tells me 'don't eat peanuts' and I love peanuts...I asked her why she said something I didn't understand, but I stopped eating peanuts, and same thing with milk, she said stop eating dairy so I stopped eating yogurt and milk." PD05 
When discussing food intake and dietary choices, many participants were not sure that their dietary intake changed as a result of having PD. Some did not feel having PD impacted their food intake, dietary choices or preferences. Several participants could not distinguish if changes they experienced with food were a result of having PD or just a natural part of the aging process.

- "I generally felt better on the Keto diet because I lost a little weight, but, um, as far as Parkinson's symptoms l'm not aware."-PD02

- 'No, I don't eat a lot, but I don't know if that's Parkinson's or not. Indirectly it probably is, because I don't have my smeller"-PD16

The final theme related to the category Other Nutrition Concerns was Dietary Needs of Caregivers. Several interviews revealed caregivers had their own dietary concerns that may differ from the PwPD or were managing their own health conditions that required dietary modifications, including neurological conditions such as multiple sclerosis and essential tremor. Many caregivers were also the ones buying and preparing foods or impacted by their loved one's dietary challenges.

- "I feel really badly for [him] because he has got nothing to eat because just being in the kitchen thinking about food is awful but he's not wasting away, so..." -PD15

- 'I mean I call him almost every day 'got any ideas for dinner?', but I think for the most part it all falls on me. I think that he really does think that if he ate better, he might feel better, so again it's on me."-CG18

Perceptions of Diet. In total 113 phrases were coded within the category Perceptions of Diet. Phrases coded as Perceptions of Diet fit into three subthemes (Figure 1). Of the 72 phrases coded as Perceived Usefulness of Diet for Managing PD, $73.6 \%$ of these phrases described how dyads thought 
dietary choices were useful for Managing PD, while $19.4 \%$ of phrases coded described how dyads were not sure or neutral regarding the role diet plays for managing PD. Only $6.9 \%$ of phrases coded described how participants thought a healthy diet was not useful for managing PD. As a result, there were mixed perceptions as to how useful diet can be for managing PD. The following quotes are examples of responses to the question, "How important is it to follow an eating plan for managing PD."

- 'It's very important, I don't know if you would call it an eating plan, but it's important to know what you are going to eat and when you are going to eat it and figure out how to back up from what time it is now and to dinner and when you should start taking your medicines and put it in proper order." -PD12

- "I think more science needs to be done there, I think there needs to be some things that they find that [some foods] are especially good...some fruit that comes from Asia somewhere."-CG16

For the sub-theme, Perceived Usefulness of Diet for Managing Health, $85 \%$ of phrases coded indicated participants found following a diet useful for managing overall health and only $15 \%$ of phrases coded indicated participants found following a diet to be useless for managing overall health.

- "I don't count calories. I eat what I eat."-PD04

"But you need to! We need to be more cognizant of the caloric intake because it affects how much you weigh, and if you lost 15 pounds, your core would be much more manageable. As would mine be! ...one isn't independent as each other. So caloric intake does count! You just don't think it does." -CG04

A third theme within the category of Perceptions of Diet was Perception of Own Diet Quality. Of the 21 phrases coded, $76 \%$ of phrases coded described participants who perceived they had good dietary quality, while $14 \%$ 
of phrases coded were linked to participants who perceived they had poor diet quality. Only 2 interviews and $9.5 \%$ of the phrases coded reflected that participants recognized that their diet could be improved or that they were interested in making improvements in their diet quality; these phrases were coded as Neutral.

- "Only that I know that I should have more fruits and vegetables."-PD15

- "We need a healthier diet. We do eat like fish once a week at least, but we also eat like pizza, you know... But yeah I think we need to eat healthier, we definitely need to eat healthier. He tries to, he's a lot better at it than I am." -CG18

\section{Integration of Quantitative and Qualitative Data}

The frequency of themes coded within the category Dietary Concerns Related to PD Sequelae were compared with HEI-2015 scores to detect if any pattern between self-reported concerns and diet quality existed. Given the large variation between HEl-2015 scores and Self-Reported Nutrition Concerns Related to PD sequelae. Several PwPD reported a change in taste, including a preference for sweeter foods. However, about $50 \%$ of participants had high added sugar moderation scores, indicating that their diets are low in added sugar. Sodium scores indicate excess salt intake may be a concern for PwPD and caregivers. Higher salt intake may be attributed to change in taste as well as the intake of convenience and processed foods.

\section{Discussion}

This study is the first to examine PwPD's and caregivers' diet quality in conjunction with self-reported dietary concerns related to managing PD. Findings from this study indicate PwPD and caregivers have poor diet quality 
as well as nutrition concerns regarding PD sequelae and their own nutrition literacy. Qualitative analyses revealed dyads also believe that a healthy diet may be important for managing PD and overall health. However, there was no pattern between HEI-2015 scores and Nutrition Concerns Related to PD Sequelae. Findings from this study can be used to provide tailored nutrition counseling and inform nutrition interventions among PwPD and caregivers.

This study adds to the body of literature of dietary quality and PD management by providing objective dietary quality data which is scarce. Our study supports the caregiver as an integral part of the care provided to PwPD and without including caregivers' dietary management for PD may be incomplete. Total HEI-2015 scores of both PwPD and their caregivers was comparable to the average $\mathrm{HEI}-2015$ scores for Americans of 59 , or an $\mathrm{F} .50$ Compared to the national HEI-2015 component for Americans, dyads scores indicate PwPD and caregivers are doing a better including whole grains in their diets and limiting added sugar intake. ${ }^{50}$ Mean added sugar component scores were similar to American older adult scores. ${ }^{50}$ Dyads may need some education on how to incorporate protein and healthy fat into their diets as well as to minimize sodium intake.

Few studies have examined diet quality and patterns among PwPD but nutrition status has been extensively examined. Past research shows that PwPD have compromised nutrition status and are at nutrition risk ${ }^{3,13,24}$. Our findings were similar to key findings from Cassani et $a^{25}$, who found no differences in summary scores assessing adherence to the Mediterranean diet 
between PwPD and controls; results also showed both groups could be making dietary choices that better adhere with the Mediterranean diet. Marczewska et al. ${ }^{26}$ examined daily intake among PwPD and their spouses and found no difference in average daily energy intake, but did find differences in individual food groups such as vegetable protein and carbohydrates. Our study adds to this research by looking at over all dietary quality and adherence to the DGAs, rather than in terms of individual nutrients.

Obtaining self-reported nutrition concerns of PwPD and their caregivers adds to the body of patient-centered care research. Dyads expressed dietary concerns related to PD sequelae, such as mealtime related issues, change in appetite, and gastrointestinal issues. Findings from our study indicate participants want to increase nutrition literacy, have pre-existing perceptions around nutrition intake, and are using complimentary care services. These findings expands upon previous research which has found PwPD and caregivers want to be involved in the communication and decision making of their care.${ }^{51}$ Past research shows high levels of education and treatment of a movement disorder specialist were significantly related to PwPD using complementary health service. ${ }^{52}$ The majority of dyads in our study had at least a college degree and most PwPD were receiving treatment from a movement disorders specialist. Young Shin et al. ${ }^{52}$ found exercise and vitamin supplements were most commonly reported forms of complimentary healthcare. Many of our participants reported in their medical history questionnaire to be taking various supplements to manage health. 
In addition to better understanding dietary quality and concerns among this population, our study expands upon previous research describing the day-today challenges of navigating PD and sheds light onto how this may impact not only diet quality but overall health-related QOL. The emerging theme from our study, Managing Life, supports previous literature published by Smith and Shaw, ${ }^{37}$ which described the existential challenge shared by PwPD and their loved ones. Finally, exploring these self-reported nutrition concerns from this study may be able to help better understand the diet quality of participants in this study is warranted.

The lack of pattern consistency between HEI-2015 scores and the number of self-reported nutrition concerns related to PD sequelae may indicate our participants may be in varying stages of the Transtheoretical Model. ${ }^{53}$ For instance, Dyad 16, the dyad with the highest HEI-2015 scores, also had the highest number of phrases coded within Self-Reported Nutrition Concerns Related to PD Sequelae (Table 4). This occurrence may indicate that this dyad was actively making dietary changes to minimize nutrition concerns and more cognizant of their dietary choices compared to other participants. The dyad with the second highest HEI-2015 scores, Dyad 4, had only two phrases coded within Self-Reported Nutrition Concerns Related to PD Sequelae. This suggests this dyad was actively managing their nutrition concerns. Most dyads had HEI-2015 scores ranging from 43-70 and 9-12 phrases coded around nutrition concerns. Findings suggest assessing dyads readiness to make dietary changes could be beneficial for helping to improve diet quality. ${ }^{53}$ 
Self-reported perceptions and actual dietary patterns may vary due to alterations in perception that PwPD experience as part of the disease. These findings, combined with poor diet quality, suggest this population can benefit from nutrition education to improve diet quality and nutrition knowledge.

Low adequacy and moderation HEI-2015 component scores may shed light on some of the self-reported nutrition concerns that came up during semistructured dyadic interviews. The sub-theme Mealtime Related Issues coded in dyadic interviews may account for low dietary scores and influence dietary choices and help to explain overall low HEl-2015 total and component scores among dyads. Our participants were not meeting maximum adequacy in areas of fruits, vegetables, and whole grains, sources of fiber which may help to reduce self-reported complains of constipation among our population. Future interventions should help PwPD obtain adequate fiber, manage dietary protein intake and reduce consumption of sodium and saturated fat. Low dairy intake among PwPD may be a concern. The literature shows that osteoporosis and osteopenia can impact up to $91 \%$ of women and $61 \%$ of men with PwPD. ${ }^{54}$ Most of our participants were taking supplements, including supplements to support bone health, which could be why nutrition for bone health did not appear as a self-reported nutrition concern. Understanding nutrition concerns among dyads may be a way to address nutrients of concern unique to PD and in turn help improve diet quality scores. Similar HEI-2015 scores between caregivers and PwPD indicate that caregivers can also benefit from nutrition education, especially since the majority of the dyads were living together. 
Findings from this study can also help to inform digital nutrition interventions, which are lacking in this population. Tailored nutrition counseling by a registered dietitian that includes both the PWPD and caregiver could be beneficial for this population. Nutrition counseling can help to increase nutrition knowledge and promote dietary behavior change. ${ }^{55,56}$ Nutrition interventions should address the nutrition concerns discussed in semi-structured interviews among PwPD and caregivers as a way to help improve diet quality seek to help improve diet quality. Subsequently, including caregivers could optimize the health of the patient-caregiver dyad. ${ }^{27,28}$ In addition to managing PD, both PwPD and caregivers were dealing with other health conditions that may need dietary attention and can help reduce caregiver strain. Couple-oriented interventions improve spousal coping strategies, promote disease related stress and anxiety management, increase self-efficacy, and help couples manage changes caused by PD. ${ }^{57}$

Strengths and Limitations. This novel study had several strengths. Several measures were taken to ensure accuracy of the dietary intake information. To reduce recall bias, we used the multiple pass method, the gold standard for collecting dietary recall information. ${ }^{41}$ Enrolling both PwPD and caregivers also helped increase accuracy of dietary information. For example, several male participants, both PwPD and caregivers, needed assistance from their spouse to report dietary intake during 24-hour recalls. To measure diet quality, $\mathrm{HEI}-2015$ was used, which is a validated, comprehensive measure of dietary intake that is linked to adherence of 2015-2020 DGAs. ${ }^{42}$ The mixed- 
method study design collected both quantitative and qualitative data that provides an in-depth understanding of dyads' diet quality and nutrition concerns that may help explain diet quality. The use of semi-structured dyadic interviews promotes interaction between participants to provide detailed information regarding their nutrition concerns and PD management. ${ }^{58}$ Finally, this study promotes the inclusion of PwPD and their caregiver as part of the healthcare team and the concept of patient-and caregiver-centered care for managing PD.

Despite study strengths, this study has several limitations. Findings from our study are not generalizable to all PwPD. Most of our participants were educated and all of them identified as Caucasian. Cognitive status was an inclusion criterion to enroll in this study and as a result most of our participants had low reliance on caregivers and were able to perform activities of daily living with minimal assistance. However, research supports including caregivers early at disease onset and encourages caregivers to partner with healthcare providers to help cope with disease progression. ${ }^{59} \mathrm{~A}$ limitation of the HEl-2015 assessment measure is that it does not assess fluid intake. Many of our participants complained about a change in thirst, which could impact negatively impact hydration status. Cassani et $\mathrm{al}^{25}$ found that PwPD drank significantly less fluid compared to healthy controls. Finally, requests for personal health information, including disease stage, were sent to physicians' office but we were unable to obtain this information for all 20 PwPD. 


\section{Future Research.}

Concerns about low nutrition literacy in addition to poor diet quality among study participants may be explained by dyads' limited access to nutrition education. During semi-structured interviews, when asked "Where do you get information about foods and diets for people with PD?" most dyads described getting information from support groups or attending one or two education sessions. Only one PwPD reported working with a dietitian overtime to manage nutrition and PD. Future research should explore barriers PwPD and caregivers have for accessing nutrition professionals. Future work should also explore ways to improve access to nutrition professionals through expanding other healthcare professionals' knowledge of nutrition services as well as promote policy changes to expand insurance coverage for medical nutrition therapy among PwPD. This is important since several participants reported obtaining dietary recommendations that were not evidenced-based or from non-nutrition experts.

\section{Conclusion}

This mixed-methods study focused on describing the diet quality and self-reported nutrition concerns of PwPD and their caregivers. Healthy Eating Index-2015 scores indicate PwPD and caregivers have low adherence to current dietary guidelines and present with dietary concerns related to PD. Poor diet quality and the self-reported nutrition concerns indicates dyads can benefit from nutrition education and support the inclusion of caregivers and nutrition professionals for managing nutrition and health. 


\section{Chapter 2 Tables and Figures}

Figure 1: Flow Chart of Qualitative Data by Major Categories and SubThemes

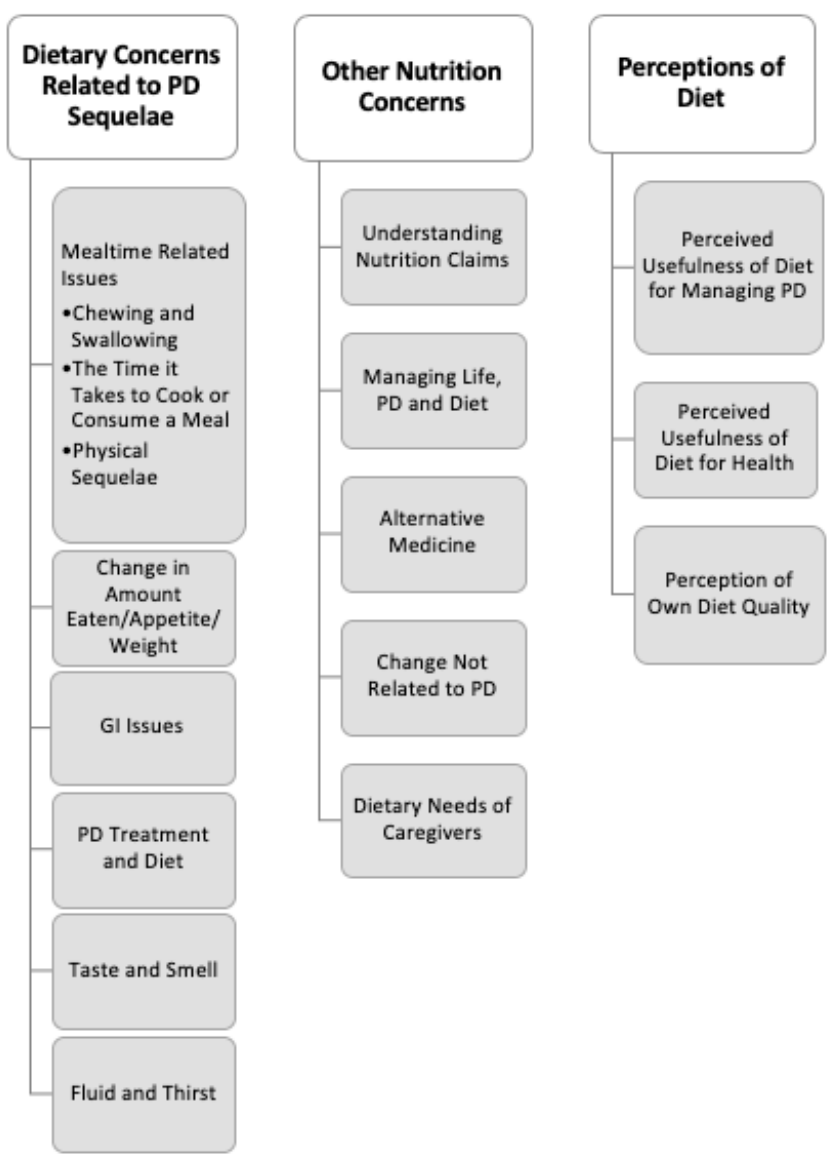

Figure 1 Abbreviations: $P D=$ Parkinson's disease; $G /=$ Gastrointestinal Issues 
Figure 2: Frequency of PwPD and Caregivers with $\geq 80 \%$ Adequacy and Moderation Component Scores

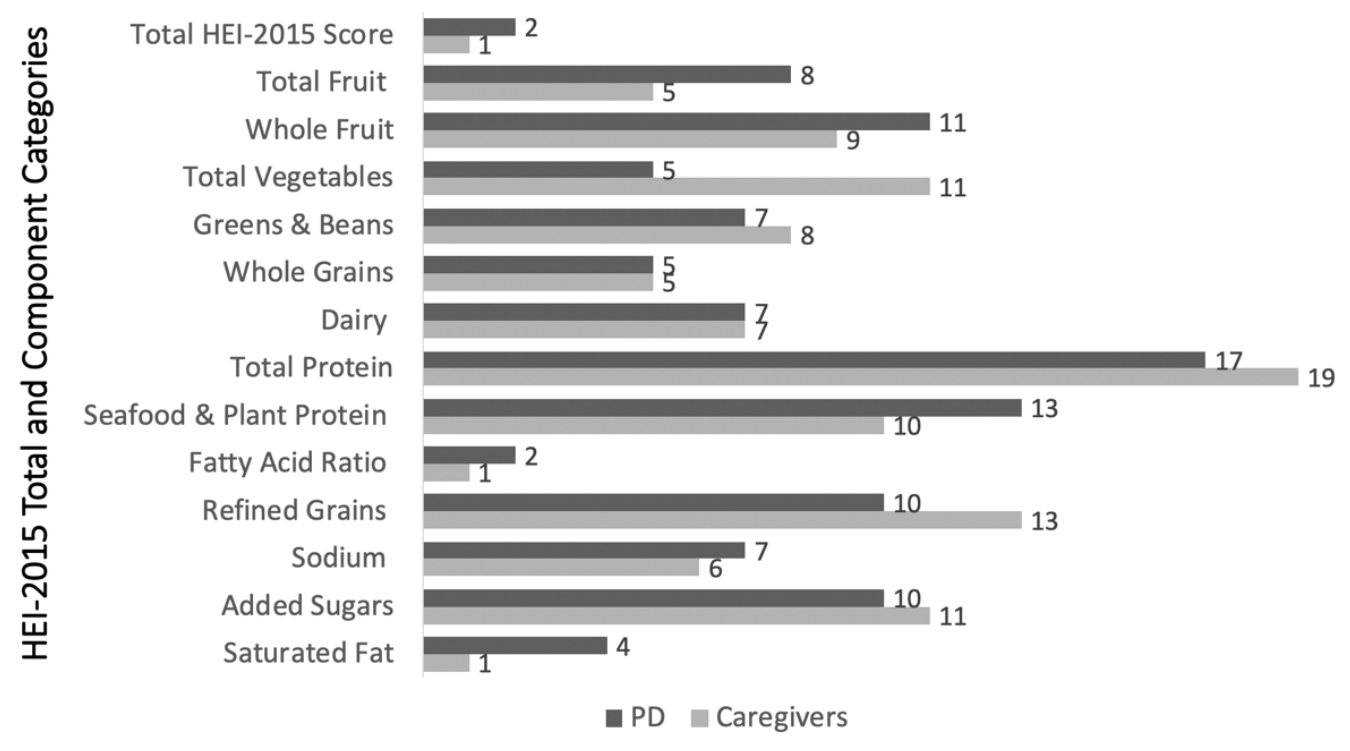


Table 1: Descriptive Characteristics of PwPD and Caregivers

\begin{tabular}{|c|c|c|c|c|}
\hline Characteristics & PwPD & Caregivers & Range & $P$ \\
\hline Age (years) & $69.7 \pm 9.2$ & $66.4 \pm 13.0$ & $39-89$ & 0.4 \\
\hline $\begin{array}{l}\text { Gender } \mathrm{n}(\%) \\
\text { Male } \\
\text { Female }\end{array}$ & $\begin{array}{c}13(65) \\
7(35)\end{array}$ & $\begin{array}{c}4(20) \\
16(80)\end{array}$ & NA & 0.01 \\
\hline $\begin{array}{ll}\text { Education n(\%) } \\
\qquad \text { HS Diploma/Some } \\
\text { College } \\
\text { - Technical } \\
\text { Training/Trade } \\
\text { School/Associates } \\
\text { - } \\
\text { ZCollege Degree or } \\
\text { Greater }\end{array}$ & $\begin{array}{l}6(30) \\
3(15) \\
11(55)\end{array}$ & $\begin{array}{r}1(5) \\
5(25) \\
14(70)\end{array}$ & NA & 0.11 \\
\hline $\begin{array}{cl}\text { Employment Status } \mathrm{n}(\%) \\
\text { - Retired } \\
\text { - Part Time } \\
\text { - } \\
\text { Full Time }\end{array}$ & $\begin{array}{l}15(75) \\
3(15) \\
2(10)\end{array}$ & $\begin{array}{l}10(50) \\
2(10) \\
8(40)\end{array}$ & NA & 0.09 \\
\hline Years Since Diagnosis & $7.6(5.4)$ & NA & $\begin{array}{c}0.33- \\
18.0\end{array}$ & NA \\
\hline T-MoCA & $19.8 \pm 1.5$ & $20.4 \pm 1.1$ & $18-22$ & 0.2 \\
\hline $\begin{array}{c}\text { Caregiver Relationship (\%) } \\
\text { - Spouse/Partner } \\
\text { - Child } \\
\text { - } \text { Friend }\end{array}$ & NA & $\begin{array}{c}17(85) \\
2(10) \\
1(5)\end{array}$ & NA & NA \\
\hline
\end{tabular}

Independent samples t-tests and chi square analyses performed. A statistical significance was indicated at a $\mathrm{p}$-value of $<0.05$. Abbreviations: T-MoCA=Telephone Montreal Cognitive Assessment. 
Table 2: Diet Assessment of PwPD and Caregivers

\begin{tabular}{|l|c|c|c|}
\hline Diet Assessment Variables & $\begin{array}{c}\text { PwPD } \\
(\mathbf{n = 2 0})\end{array}$ & $\begin{array}{c}\text { Caregivers } \\
(\mathbf{n = 2 0})\end{array}$ & Range \\
\cline { 1 - 2 } Anthropometrics & $66.1 \pm 3.3$ & $65.1 \pm 3.8$ & $57.5-70.7$ \\
\hline Height (in) & $77.3 \pm 19.9$ & $77.4 \pm 16.4$ & $35.6-116.6$ \\
\hline WMeight (kg) $\left(\mathrm{kg} / \mathrm{m}^{2}\right)$ & $27.1 \pm 5.4$ & $28.3 \pm 5.7$ & $15.4-43.1$ \\
\hline \% Body Fat (n=33) & $29.1 \pm 8.8$ & $34.2 \pm 7.8$ & $13-49$ \\
\hline Waist Circumference (in) & $39.4 \pm 6.0$ & $39.4 \pm 6.3$ & $25.2-52.4$ \\
\hline Nutrient Intake & & & \\
\hline Kcalorie Intake & $1887.4 \pm 728.1$ & $1752.7 \pm 465.8$ & $908.8-3344.4$ \\
\hline \% Calories from CHO & $44.2 \pm 9.6$ & $41.5 \pm 8.0$ & $24.4-57.0$ \\
\hline \% Calories from Fat & $36.7 \pm 6.7$ & $37.7 \pm 7.2$ & $24.7-50.3$ \\
\hline \%Calories from Protein & $15.9 \pm 4.1$ & $17.2 \pm 3.8$ & $10.6-27.11$ \\
\hline \% Calories from Sat Fat & $12.8 \pm 3.1$ & $13.3 \pm 2.8$ & $6.6-18.5$ \\
\hline
\end{tabular}

Analyses did not include dietary supplements; independent samples t-tests performed and a statistical significance was indicated at a $p$-value of $<0.05$. Mann-Whitney test for non-normally distributed data ( $\mathrm{g} / \mathrm{kg}$ protein; \% calories from protein); \%BF n=16 PwPD and 17 CG (implantable devices such as DBS and Pacemaker) Abbreviations: DRI=dietary reference intake; BMI=body mass index, Avg=average 
Table 3: HEl-2015 Scores of PwPD and Caregivers

\begin{tabular}{|l|c|c|c|}
\hline HEI-2015 Scores & PwPD & Caregivers & Range \\
\hline Total Score (0-100) & $58.3 \pm 12.4$ & $58.1 \pm 10.6$ & $37.7-83.4$ \\
\hline Adequacy Component: & & & \\
\hline Total Fruit (0-5) & $2.9 \pm 1.9$ & $2.5 \pm 1.7$ & $0.0-5.0$ \\
\hline Whole Fruit (0-5) & $3.3 \pm 2.0$ & $3.2 \pm 1.9$ & $0.0-5.0$ \\
\hline Total Vegetables (0-5) & $2.6 \pm 1.9$ & $3.4 \pm 1.6$ & $0.1-5.0$ \\
\hline Greens and Beans (0-5) & $2.5 \pm 1.9$ & $2.6 \pm 2.2$ & $0.0-5.0$ \\
\hline Whole Grains (0-10) & $4.8 \pm 3.4$ & $5.0 \pm 3.1$ & $0.0-10.0$ \\
\hline Dairy (0-10) & $5.9 \pm 3.0$ & $7.1 \pm 2.4$ & $1.1-10.0$ \\
\hline Total Protein (0-5) & $4.6 \pm 0.7$ & $4.7 \pm 0.6$ & $2.8-5.0$ \\
\hline Sea Food and Plant Protein (0-5) & $3.6 \pm 1.9$ & $2.9 \pm 2.2$ & $0.0-5.0$ \\
\hline Fatty Acid Ratio (0-10) & $3.9 \pm 2.9$ & $3.0 \pm 2.3$ & $0.0-10.0$ \\
\hline Moderation Component: & & & \\
\hline Refined Grains (0-10) & $7.0 \pm 3.1$ & $8.0 \pm 2.6$ & $0.0-10.0$ \\
\hline Sodium (0-10) & $5.5 \pm 3.7$ & $4.8 \pm 3.8$ & $0.0-10.0$ \\
\hline Added Sugars (0-10) & $7.6 \pm 2.5$ & $7.4 \pm 2.6$ & $1.2-10.0$ \\
\hline Saturated Fat (0-10) & $4.1 \pm 3.4$ & $3.6 \pm 2.8$ & $0.0-10.0$ \\
\hline
\end{tabular}

Group differences ran using independent samples t-tests and Mann-Whitney $U$ tests, $p$-value of $<0.05$ indicated statistical significance. Abbreviations: HEl-2015=healthy eating index-2015 
Table 4: Number of Times Nutrition Concerns Related to Parkinson's Disease Sequelae Were Coded During Dyadic Interviews: A Side-By-Side Comparison with HEI Scores.

\begin{tabular}{|c|c|c|c|}
\hline \multirow[b]{2}{*}{ Dyads } & \multirow{2}{*}{$\begin{array}{l}\text { Total Nutrition } \\
\text { Concerns Related } \\
\text { to PD-Sequelae }\end{array}$} & \multicolumn{2}{|c|}{ Total HEI-2015 Scores } \\
\hline & & $\begin{array}{l}\text { PwPD } \\
(n=20)\end{array}$ & Caregivers $(n=20)$ \\
\hline Dyad 1 & 9 & 65.7 & 43.7 \\
\hline Dyad 2 & 4 & 44.9 & 41.8 \\
\hline Dyad 3 & 1 & 63.1 & 62.8 \\
\hline Dyad 4 & 2 & 83.0 & 78.7 \\
\hline Dyad 5 & 9 & 45.1 & 51.8 \\
\hline Dyad 6 & 11 & 46.6 & 70.3 \\
\hline Dyad 7 & 10 & 48.6 & 43.7 \\
\hline Dyad 8 & 7 & 54.5 & 54.3 \\
\hline Dyad 9 & 7 & 54.3 & 54.5 \\
\hline Dyad 10 & 11 & 62.9 & 63.1 \\
\hline Dyad 11 & 11 & 63.1 & 62.9 \\
\hline Dyad 12 & 12 & 59.8 & 54.6 \\
\hline Dyad 13 & 11 & 59.3 & 56.6 \\
\hline Dyad 14 & 19 & 70.7 & 61.0 \\
\hline Dyad 15 & 13 & 46.1 & 57.2 \\
\hline Dyad 16 & 21 & 81.4 & 83.4 \\
\hline Dyad 17 & 10 & 47.0 & 57.5 \\
\hline Dyad 18 & 9 & 60.5 & 51.1 \\
\hline Dyad 19 & 5 & 71.0 & 59.3 \\
\hline Dyad 20 & 0 & 37.7 & 53.8 \\
\hline $\begin{array}{l}\text { Total } \\
\text { Phrases } \\
\text { Coded }\end{array}$ & 182 & & \\
\hline
\end{tabular}

\section{References:}

1. Fahn S. Description of Parkinson's disease as a clinical syndrome. Annals of the New York Academy of Sciences. 2003;991:1-14.

2. Borlongan CV, Burns J, Tajiri N, et al. Epidemiological survey-based formulae to approximate incidence and prevalence of neurological disorders in the United States: A meta-analysis. PloS one. 2013;8(10):e78490.

3. Barichella M, Cereda E, Pezzoli G. Major nutritional issues in the management of Parkinson's disease. Movement Disorders. 2009;24(13):18811892.

4. Barichella M, Cereda E, Madio C, et al. Nutritional risk and gastrointestinal dysautonomia symptoms in Parkinson's disease outpatients hospitalised on a scheduled basis. The British Journal of Nutrition. 2013;110(2):347-353. 
5. Kowal SL, Dall TM, Chakrabarti R, Storm MV, Jain A. The current and projectedd economic burden of Parkinson's disease in the United States. Movement Disorders. 2013;28(3):311-218.

6. Lees AJ, Hardy J, Revesz T. Parkinson's disease. Lancet. 2009;373(9680):2055-2066.

7. Storch A, Schneider CB, Wolz M, et al. Nonmotor fluctuations in Parkinson disease: severity and correlation with motor complications. Neurology. 2013;80(9):800-809.

8. Cilia R, Cereda E, Klersy C, et al. Parkinson's disease beyond 20 years. $J$ Neurol Neurosurg Psychiatry. 2014;86(8):849-855.

9. Boersma I, Jones J, Carter J, et al. Parkinson disease patients' perspectives on palliative care needs: What are they telling us? Neurology Clinical practice. 2016;6(3):209-219.

10. Vikdahl M, Domellof ME, Forsgren L, Haglin L. Olfactory function, eating ability, and visceral obesity associated with MMSE three years after Parkinson's disease diagnosis. The Journal of Nutrition, Health \& Aging. 2015;19(9):894-900.

11. Wills AA, Perez A, Wang J, et al. Association between change in body mass index, Unified Parkinson's Disease Rating Scale Scores, and survival among persons with Parkinson disease: Secondary analysis of longitudinal data From NINDS exploratory trials in Parkinson disease long-term study 1. JAMA Neurology. 2016;73(3):321-328.

12. Lorefalt B, Ganowiak W, Palhagen S, Toss G, Unosson M, Granerus AK. Factors of importance for weight loss in elderly patients with Parkinson's disease. Acta Neurologica Scandinavica. 2004;110(3):180-187.

13. Sheard JM, Ash S, Silburn PA, Kerr GK. Prevalence of malnutrition in Parkinson's disease: a systematic review. Nutrition Reviews. 2011;69(9):520532.

14. Leroi I, McDonald K, Pantula H, Harbishettar V. Cognitive impairment in Parkinson disease: Impact on quality of life, disability, and caregiver burden. Journal of Geriatric Psychiatry and Neurology. 2012;25(4):208-214. 
15. The National Alliance for Caregiving (NAC) and the AARP Public Policy Institute. Caregiving in the US. 2015;

http://www.aarp.org/content/dam/aarp/ppi/2015/caregiving-in-the-unitedstates-2015-report-revised.pdf Accessed January, 2017.

16. Polenick CA, DePasquale N. Predictors of Secondary Role Strains Among Spousal Caregivers of Older Adults With Functional Disability. The Gerontologist. 2019;59(3):486-498.

17. Shulman LM, Gruber-Baldini AL, Anderson KE, et al. The evolution of disability in Parkinson disease. Movement Disorders. 2008;23(6):790-796.

18. Winter JE, McNaughton SA, Nowson CA. Older Adults' Attitudes to Food and Nutrition: A Qualitative Study The Journal of Aging Research \& Clinical Practice 2012;5:114-119.

19. Nutrition and Your Health. 2015-2020 Dietary Guidelines for Americans. Washingnton, DC: US Government Printing Office. Home and Garden Bulletin No.232;2015

20. Kirkpatrick SI, Reedy J, Krebs-Smith SM, et al. Applications of the Healthy Eating Index for Surveillance, Epidemiology, and Intervention Research: Considerations and Caveats. Journal of the Academy of Nutrition and Dietetics. 2018;118(9):1603-1621.

21. Rezak M. Nutritional Issues in Parkinson's Disease. American Parkinson's Disease Association. 2017.

22. Baroni L, Bonetto C, Tessan F, et al. Pilot dietary study with normoproteic protein-redistributed plant-food diet and motor performance in patients with Parkinson's disease. Nutritional Neuroscience. 2011;14(1):1-9.

23. Taghizadeh M, Tamtaji OR, Dadgostar E, et al. The effects of omega-3 fatty acids and vitamin $\mathrm{E}$ co-supplementation on clinical and metabolic status in patients with Parkinson's disease: A randomized, double-blind, placebocontrolled trial. Neurochem Int. 2017;108:183-189.

24. LoBuono DL, Taetzsch AG, Lofgren IE, Xu F, Delmonico MJ, Mahler L. Cognitive status and cardio-metabolic risk of patients with acquired brain injury and Parkinson's disease. Disability and Health Journal. 2015;9(1):134139. 
25. Cassani E, Barichella M, Ferri V, et al. Dietary habits in Parkinson's disease: Adherence to Mediterranean diet. Parkinsonism \& Related Disorders. 2017;42:40-46.

26. Marczewska A, De Notaris R, Sieri S, Barichella M, Fusconi E, Pezzoli G. Protein intake in Parkinsonian patients using the EPIC food frequency questionnaire. Movement Disorders. 2006;21(8):1229-1231.

27. Martinez-Martin P, Arroyo S, Rojo-Abuin JM, et al. Burden, perceived health status, and mood among caregivers of Parkinson's disease patients. Movement Disorders. 2008;23(12):1673-1680.

28. Beach SR, Schulz R. Family caregiver factors associated with unmet needs for care of older adults. Journal of the American Geriatrics Society. 2016.

29. Carter JH, Lyons KS, Lindauer A, Malcom J. Pre-death grief in Parkinson's caregivers: A pilot survey-based study. Parkinsonism \& Related Disorders. 2012;18 Suppl 3:S15-18.

30. Berry RA, Murphy JF. Well-being of caregivers of spouses with Parkinson's disease. Clinical Nursing Research. 1995;4(4):373-386.

31. Avgerinou C, Bhanu C, Walters K, et al. Exploring the Views and Dietary Practices of Older People at Risk of Malnutrition and Their Carers: A Qualitative Study. Nutrients. 2019;11(6).

32. Bhanu C, Avgerinou C, Kharicha K, et al. 'I've never drunk very much water and I still don't, and I see no reason to do so': a qualitative study of the views of community-dwelling older people and carers on hydration in later life. Age and Ageing. 2019;49(1):111-118.

33. Martignoni E, Citterio A, Zangaglia R, et al. How parkinsonism influences life: the patients' point of view. Neurological Sciences. 2011;32(1):125-131.

34. Navarta-Sanchez MV, Caparros N, Fernandez MR, Diaz De Cerio Ayesa S, Ursua Sesma ME, Portillo MC. Core elements to understand and improve coping with Parkinson's disease in patients and family carers: A focus group study. Journal of Advanced Nursing. 2017.

35. Raein KL, Ortiz-Hernandez S, Benge JF. Cognitive Problems in Parkinson Disease: Perspectives and Priorities of Patients and Care Partners. Cogn Behav Neurol. 2019;32(1):16-24. 
36. Balash Y, Korczyn AD, Knaani J, Migirov AA, Gurevich T. Quality-of-life perception by Parkinson's disease patients and caregivers. Acta Neurologica Scandinavica. 2017;136(2):151-154.

37. Smith LJ, Shaw RL. Learning to live with Parkinson's disease in the family unit: An interpretative phenomenological analysis of well-being. Med Health Care Philos. 2017;20(1):13-21.

38. Leavy P. Mixed Methods Research Design. In: Research Design: Quantitative, Qualitative, Mixed Methods, Arts-Based, and Community-Based Participatory Research Approaches. New York, NY: The Guilford Press; 2017:175-176.

39. Castanho TC, Amorim L, Zihl J, Palha JA, Sousa N, Santos NC. Telephonebased screening tools for mild cognitive impairment and dementia in aging studies: A review of validated instruments. Frontiers in Aging Neuroscience. 2014;6:16.

40. Pendlebury ST, Welch SJ, Cuthbertson FC, Mariz J, Mehta Z, Rothwell PM. Telephone assessment of cognition after transient ischemic attack and stroke: Modified telephone interview of cognitive status and telephone Montreal Cognitive Assessment versus face-to-face Montreal Cognitive Assessment and neuropsychological battery. Stroke. 2013;44(1):227-229.

41. Johnson RK, Driscoll P, Goran MI. Comparison of multiple-pass 24-hour recall estimates of energy intake with total energy expenditure determined by the doubly labeled water method in young children. Journal of the American Dietetic Association. 1996;96(11):1140-1144.

42. Krebs-Smith SM, Pannucci TE, Subar AF, et al. Update of the Healthy Eating Index: HEl-2015. Journal of the Academy of Nutrition and Dietetics. 2018;118(9):1591-1602.

43. Ritchie JD, Miller CK, Smiciklas-Wright $\mathrm{H}$. Tanita foot-to-foot bioelectrical impedance analysis system validated in older adults. Journal of the American Dietetic Association. 2005;105(10):1617-1619.

44. Janssen I, Heymsfield SB, Allison DB, Kotler DP, Ross R. Body mass index and waist circumference independently contribute to the prediction of nonabdominal, abdominal subcutaneous, and visceral fat. Am J Clin Nutr. 2002;75(4):683-688. 
45. Shapira AL, Handzel R, Korczyn AD. The Lived Experience of Parkinson's Disease: A Content Analysis of Parkinson's Patients' Blogs. Isr Med Assoc J. 2017;19(11):685-690.

46. Gale NK, Heath G, Cameron E, Rashid S, Redwood S. Using the framework method for the analysis of qualitative data in multi-disciplinary health research. BMC Med Res Methodol. 2013;13:117.

47. Shosha GA. Employment of Colaizzi's Strategy in Descriptive Phenomenology: A Reflection of a Researcher. European Scientific Journal. 2012;8(27):31-43.

48. Saladana J. The Coding Manual for Qualitative Researchers. Thousand Oaks, California Sagem 2016.

49. Reedy J, Lerman JL, Krebs-Smith SM, et al. Evaluation of the Healthy Eating Index-2015. Journal of the Academy of Nutrition and Dietetics.

2018;118(9):1622-1633.

50. US Department of Agriculture. What We Eat in America/National Health and Nutrition Examination Survey, 2015-2016. Healthy Eating Index Scores - U.S. Department of Agriculture, Center for Nutrition Policy and Promotion, National Center for Health Statistics. Available from: https://fnsprod.azureedge.net/sites/default/files/media/file/HEl-2015 1516 web.pdf

51. Zizzo N, Bell E, Lafontaine AL, Racine E. Examining chronic care patient preferences for involvement in health-care decision making: The case of Parkinson's disease patients in a patient-centred clinic. Health Expectations. 2017;20(4):655-664.

52. Young Shin J, Pohlig R, T., Habermann B. Use of complimentary heatlh approaches in individuals with Parkinson's dsiease Journal of Gerontological Nursing 2017;43(2):46-54.

53. Prochaska JO, Velicer WF. The transtheoretical model of health behavior change. American journal of health promotion : AJHP. 1997;12(1):38-48.

54. Invernizzi M, Carda S, Sguazzini Viscontini G, Cisari C. Osteoporosis in Parkinson's Disease. Parkinsonism \& Related Disorders. 2009;15:339-346. 
55. Nykanen I, Rissanen TH, Sulkava R, Hartikainen S. Effects of individual dietary counseling as part of a comprehensive geriatric assessment (CGA) on nutritional status: a population-based intervention study. The Journal of Nutrition, Health \& Aging. 2014;18(1):54-58.

56. Holli BB, Beto JA. Nutrition Counseling and Education Skills for Dietetics Professionals 6th Edition ed. Baltimore, MD Lippincott Williams \& Wilkins; 2014.

57. Martire LM, Schulz R, Helgeson VS, Small BJ, Saghafi EM. Review and metaanalysis of couple-oriented interventions for chronic illness. Annals of Behavioral Medicine. 2010;40(3):325-342.

58. Morgan DL. Essentials of Dyadic Interviewing. Walnut Creek, CA: Left Coast Press, Inc 2016.

59. Roth DL, Fredman L, Haley WE. Informal caregiving and its impact on health: a reappraisal from population-based studies. The Gerontologist. 2015;55(2):309-319. 


\section{CHAPTER THREE}

Acceptance and Perception of Digital Health for Managing Nutrition in People with Parkinson's Disease and Their Caregivers and Their Digital Competence in the United States: A Mixed-Methods study

By

Dara L. LoBuono ${ }^{1}$, Kyla S. Shea ${ }^{2}$, Alison Tovar ${ }^{3}$, Skye N. Leedahl' ${ }^{4}$, Leslie Mahler $^{5}$, Furong $\mathrm{Xu}^{6}$, Ingrid Lofgren ${ }^{7}$

will be submitted to: Health and Social Care in the Community

${ }^{1}$ PhD Candidate, Department of Nutrition and Food Sciences, The University of Rhode Island, Kingston, RI 02881. Email: dara lobuono@uri.edu

2

Undergraduate Research Assistant, Department of Nutrition and Food Sciences, The University of Rhode Island, Kingston, RI 02881. Email: kyla shea@my.uri.edu

${ }^{3}$ Associate Professor, Department of Nutrition and Food Sciences, The University of Rhode Island, Kingston, RI 02881. Email: alison tovar@uri.edu

${ }^{4}$ Associate Professor, Department of Human Development and Family Studies, The University of Rhode Island, RI, 02881. Email:

skyeleedahl@uri.edu

${ }^{5}$ Associate Professor, Department of Communicative Disorders, The University of Rhode Island, RI 02811. Email: Imahler@uri.edu

${ }^{6}$ Professor, Department of Kinesiology, The University of Rhode Island, RI 02881. fxu2007@uri.edu

${ }^{7}$ Professor, Department of Nutrition and Food Sciences, The University of Rhode Island, RI, 02881. Email: ingrid lofgren@uri.edu 
Acceptance and Perception of Digital Health for Managing Nutrition in People with Parkinson's Disease and Their Caregivers and Their Digital Competence in the United States: A Mixed-Methods study

By

Dara L. LoBuono ${ }^{1}$, Kyla S. Shea ${ }^{1}$, Alison Tovar ${ }^{1}$, Skye N. Leedahl², Leslie Mahler $^{3}$, Furong $\mathrm{Xu}^{4}$, Ingrid E. Lofgren ${ }^{1}$

${ }^{1}$ Department of Nutrition and Food Sciences, ${ }^{2}$ Departmetn of Human Development and Family Studies, ${ }^{3}$ Department of Communicative Disorders, ${ }^{4}$ Department of Kinesiology, The University of Rhode Island, Kingston RI

\section{Abstract}

In order to deliver tailored nutrition education via digital mediums for people with Parkinson's disease (PwPD) and their information caregivers, this study examined the perception and acceptance of digital health for managing nutrition and health. Digital competence was also assessed. Using a mixedmethods design, qualitative data was collected through semi-structured, dyadic interviews and quantitative data through questionnaires from 20 dyads (20 PwPD and 20 caregivers). Data was collected in the Northeastern United States through home visits and phone interviews during the 2018-2019 academic year. Interview transcripts were deductively coded using the framework analysis method. Phrases related to Acceptance of digital were sub-coded into Accept, Neutral or Reject. Phrases related to perceptions of digital health were sub-coded into Perceived Usefulness, Perceived Ease of Use and Awareness of Digital Health. Quantitative data were analyzed using descriptives, independent samples t-tests and chi-square. To integrate this data, qualitative codes were transformed into variables and compared to 
digital competence scores. A mean acceptance rate for digital health was calculated through examining the mean percent of phrases coded as Accept from interview transcripts. Twenty-five (62.5\%) participants used the internet for at least 5 health-related purposes. The mean acceptance rate was $54.4 \%$. Dyads rejected digital health devices if they did not see the added benefit. The majority of phrases coded revealed participants found digital health useful, but hard to use, and about half of the phrases coded suggest dyads needed education about existing digital health mediums. There was no difference in mean digital competence scores between PwPD and caregivers (28.6 \pm 12.6$)$. Findings reveal dyads were accepting of and use technology, but not to its fullest potential. This may be attributed to perceiving technology as hard to use. This finding combined with digital competence scores, reveal education is warranted prior to providing a digital health intervention to deliver nutrition services.

Key words: Parkinson's disease, digital health, caregivers, nutrition education, mixed methods 
What is known about this topic:

- Increased disease burden with Parkinson's disease progression compromises the health of the caregiver and the person with Parkinson's

- The healthcare plan often overlooks nutrition and the caregiver in managing Parkinson's

- Digital health is an effective healthcare delivery mechanism, but little is known about how Parkinson's patients' and caregivers' perceptions of receiving nutrition and improving their own care

\section{What this paper adds:}

- Digital health and technology are convenient tools and provide Parkinson's patients and caregivers with new evidence-based knowledge on Parkinson's-related issues

- Parkinson's patients and caregivers are accepting of technology to manage nutrition, despite it being challenging.

- The digital competence scores indicate some training will be needed prior to implementing a digital health intervention 


\section{Introduction}

Parkinson's disease (PD) is an incurable, progressive neurodegenerative movement disorder that traditionally occurs in the second half of life (Fahn, 2003). Over 900,000 Americans are diagnosed with PD (Borlongan et al., 2013), and it costs the United States over $\$ 14$ billion per year (Kowal, Dall, Chakrabarti, Storm, \& Jain, 2013). This cost is related to disease-related motor (e.g., postural instability, bradykinesia, muscle rigidity, resting tremors) and non-motor sequelae (e.g., cognitive decline, change in taste in smell, constipation). Sequelae resulting from PD, also compromise dietary intake and nutritional status (Barichella, Cereda, \& Pezzoli, 2009), and warrant innovative nutrition care to help improve health outcomes (LoBuono et al., 2015). However, the unique nutrition services needed for PwPD are an under-recognized component of care (Vikdahl, Domellof, Forsgren, \& Haglin, 2015). Additionally, PwPD can have limited access to all healthcare providers due to sequelae, age, and location, even with the presence of the caregiver (Dorsey et al., 2016). The increase in informal caregiver burden as the disease progresses is grossly under-estimated; the majority of informal caregivers spend up to 40 hours per week caring for a PwPD (Parkinson's Australia Inc., 2015). As a result, an improved healthcare model that addresses nutrition and includes the caregiver is needed to facilitate PD management.

Digital health describes technologies that enhance managing and tracking health status (Thomas \& Bond, 2014), and include mediums such as videoconferencing, smart phones, internet applications, wearable devices, and online social networks. People with PD are promising candidates for digital 
health because a visual assessment is needed as part of on-going care by a team but PwPD can have limited mobility, visuospatial impairment, and decreased access to transportation (Achey et al., 2014). Digital health increases access to health and nutrition services (Meyer et al., 2019; Siddique et al., 2019; Stillerova, Liddle, Gustafsson, Lamont \& Silburn, 2016; Ventura Marra, Shotwell, Nelson, \& Malone, 2017), enhances quality of care (Espay et al, 2016), allows healthcare providers to obtain visual, objective and continuous data (Stamford, Schmidt, \& Friedl, 2015), decreases healthcare inefficiencies, offers more personalized services and social support (Attard \& Coulson, 2012; Shah et al., 2015), reduces burden and medical costs for PwPD (Dorsey et al. 2013), and offers caregiver support (Shah et al., 2015). People with PD and caregivers are receptive to using technology, especially if there is an added value, such as improving disease management (Ozanne et al. 2018; Schulz et al., 2016). However, research has not directly examined the use of digital health for managing nutrition-related PD concerns

In 2019, the World Health Organization published recommendations for implementing digital health interventions (World Health Organization, 2019). They recommend tracking a client's health status and using videoconference to complement, rather than replace, in-person health services in a standardized protocol with infrastructure that promotes patient privacy. To facilitate the adoption of mobile health technologies for PD management, the Movement Disorder Society Task Force on Technology proposes to identify clinically relevant and patient-centered digital outcomes, utilize technological 
mediums where the benefits exceed the burden for patients, and deliver a reliable intervention (Espay et al., 2019). The development of these digital nutrition services should include the views, needs and preferences of informal caregivers, as they are confronted with the evolving roles, increased responsibilities, and planning for the trajectory of PD (Ducharme et al., 2009; Espay et al., 2019).

This current study was part of a larger, cross-sectional study, which examined technology preferences and completed comprehensive nutrition assessments of PwPD and their informal caregivers (LoBuono et al., In Preparation). The purpose of this study was to examine PwPD's and their caregivers' perceptions and acceptance of digital health. This study also describes digital competence among PwPD and their caregivers.

\section{Methods}

\section{Study Design}

We used a mixed-methods, convergent design to compare and synthesize qualitative and quantitative data (Creswell and Plano Clark, 2018), and to fully capture dyads' perception and acceptance of digital health. How these self-reported experiences may inform digital competence were also examined. A mixed-methods design was selected to allow for a better understanding of the experiences that dyads have in relation to digital health and their needs and their preferences toward using technology for health- and 
nutrition-related purposes (Espay et al., 2019; Creswell and Plano Clark, 2018).

Semi-structured, dyadic interviews and questionnaires were used to collect qualitative and quantitative data from PwPD $(n=20)$ and their informal caregivers $(n=20)$. The PwPD and their informal caregiver were interviewed together, but questionnaires related to digital competence and technology use were completed individually. Ethical approval for this study was provided by the University of Rhode Island's Institutional Review Board (HU1819-001).

\section{Theoretical Framework}

The structure of this mixed-method study and interpretation of results were based on two theories. The technology acceptance model (TAM) provides a basis for understanding external factors that influence ender users' perceptions, attitudes, and intentions to use technology (Davis, Bagozzi, \& Warshaw, 1989. This study concentrated on the early stages of the development of digital health nutrition services, in which PwPD and caregivers provided personal opinions and preferences to inform the creation of a userfriendly, evidenced-based, digital nutrition service. The inclusion of informal caregivers is based on the emerging middle-range theory of transitions (Meleis, Sawyer, Im, Hilfinger, Messias, \& Schumacher, 2000). A transition is the change from one state or condition to another, and includes life development stages, like progressing through a disease and becoming an informal caregiver. Collecting data from PwPD and caregivers provides a more complete perspective to better understand the transition dyads face as the role 
of the caregiver evolves and the disease condition progresses, especially in relation to dietary management (Meleis et al., 2000).

\section{Sampling, Recruitment and Eligibility}

Study recruitment and data collection went from October 2018 through April 2019. Emails, flyers and announcements at support groups for PwPD and community centers were used to recruit participants. Prior to the first study visit, dyads completed an informational phone call about the study and were screened for eligibility. Eligibility criteria were, both PwPD and their caregiver had to be community-dwelling, 18+ years old, and English-speaking, and both had to participate. All participants needed to score $\geq 18$ on the Telephone Montreal Cognitive Assessment (T-MoCA), which is a cognitive screening tool (Castanho et al., 2014; Pendlebury et al., 2013). Transportation or prior technology use was not required to participate. Both PwPD and caregivers completed the informed consent process, and signed consent forms and each participant received a signed copy of the consent form.

Twenty-five dyads expressed interest. Five dyads did not continue with the study due to scheduling conflicts or low T-MoCA scores. Eighteen dyads were eligible, enrolled and interviewed. Two of these dyads included couples who were both living with PD and identified as each other's informal caregivers and were double counted as a PwPD and a caregiver. As a result, 20 dyads were included in analyses. Dyads were from Rhode Island, Massachusetts, New York, and Connecticut. We aimed to interview up to 20 dyads as previous research among PwPD and caregivers indicated this is where data 
saturation was reached (Boersma et al., 2016; Zizzo, Bell, Lafontaine, \& Racine, 2017). Saturation was reached in this study after the fourteenth interview.

\section{Qualitative Data Collection and Analysis}

The 24-question moderator guide, informed by the previous literature and the research team was organized to capture three main domains: PD and Diet, Accessibility of Nutrition and Health Information, and Digital Health for PD. From these domains, participants' acceptance and perception were assessed. A copy of the moderator guide is provided in Appendix N. Prior to starting the study, interviews were piloted with two dyads and questions were modified based on participant feedback. Interviews were conducted in the participants' homes (facilitated by DL) and were audio-recorded using a digital recorder. The mean interview length was 39 minutes (range 21-64 minutes).

Operational definitions of terms (technology, digital health, smart phones, smart watches, apps, videoconferencing) were provided during interviews. Photo prompts were used to help describe different technological devices and digital health tools and this was particularly important for understanding acceptance of devices.

Qualitative data was analyzed using deductive and inductive reasoning. Transcripts were deductively-coded using the framework analysis method (Gale, Health, Cameron, Rashid, \& Redwood, 2013), which is a seven-stage, systematic procedure that has been used previously in healthcare research and is often used when answering specific research questions. Transcripts 
were inductively-coded using Colaizzi's Strategy in Descriptive

Phenomenology (Shosha, 2012) to identify emerging themes; this

interpretative approach draws an understanding of participants' 'lived

experiences" of living with PD (Hycner, 1985).

The following steps were taken to analyze the data. Recordings were transcribed verbatim and DL checked transcripts for accuracy (stage 1). Transcripts were divided into three batches. DL and a trained research assistant (KS) analyzed one batch at a time. Separately, DL and KS coded for the following overarching a priori themes related to digital health: perception (perceived usefulness, perceived ease of use, awareness of digital health, image of technology) and acceptance (accept, neutral, reject). Themes related to perception and acceptance were identified a-priori adopting components of TAM (Davis, Bagozzi, \& Warshaw, 1989). The model hypothesizes that perceived usefulness and perceived ease of use jointly determine acceptance, which can influence intention to use and actual behavioral use of technology (Davis, Bagozzi, \& Warshaw, 1989).

Before coding individually, KS and DL read through an entire batch of transcripts and developed a list of initial impressions and themes and then coded a priori (stage 2). Both DL and KS coded one transcript from the batch independently and in duplicate (stage 3). The two researchers compared and reconciled coding, and there was a strong agreement between authors on the transcripts reviewed. During this discussion the two researchers developed a working analytical framework and agreed upon which codes to use on the 
remaining transcripts (stage 4). The transcripts were uploaded to NVivo12 (QSR International Pty Ltd, AU) and coded separately by DL and KS. An intercoder reliability was calculated and found acceptable (Bazeley and Jackson, 2013; Saladaña, 2016), with an agreement $>80 \%$ achieved for each overarching theme. DL and KS met to discuss coding differences and came to consensus. The research committee and DL met to collapse and finalize themes (stage 5). Data was then charted into framework matrices using NVivo12 to display codes within each theme (stage 6). The number of phrases coded within themes were summed to calculate frequencies and percentages. Data was interpreted, and connections related to digital competence and technological preferences of PwPD and their caregivers were made (stage 7). Although a priori themes helped to inform this framework, a phenomenological and iterative approach throughout each stage of the analyses was also taken to identify emerging themes that may impact technology use among this population. Both DL and KS contributed to the framework development with the advisement of the dissertation committee.

\section{Quantitative Data Collection and Analysis}

Both PwPD and caregivers completed demographics, medical history, dietary screening tool (Bailey et al., 2007), and digital competence (Measuring Digital Health Skills across the EU: EU Wide Indicators of Digital Competence, "European Commission, 2014) and technology use survey ("2015 Health Information National Trends Survey", Kontos, Blake, Chou, \& Prestin, 2014; National Cancer Institute, 2015; Nelson et al., 2004). The specific questions used in each questionnaire are provided in Appendices P and Q. 
Questionnaires examining technology use and digital health use, combined with qualitative data, informed dyads' acceptance of digital health. Questions related to where participants accessed health information and ease of obtaining health information was integrated with qualitative perception data.

To further describe the population, PwPD completed the 39-item Parkinson's Disease Questionnaire was administered (PDQ-39, Jenkinson, Fitzpatrick, Peto, Greenhall, \& Hyman, 1997) to asses health-related quality of life, and caregivers completed the Multidimensional Caregiver Strain Index (MCSI) to assess caregiver burden (Stull, 1996). Height and weight were collected, and body mass index was calculated.

Quantitative data was analyzed using SPSSv26 (IBM Corp. Summers, NY). All data were normally distributed. Categorical variables are represented as frequencies and percentages, and continuous variables are reported as mean and standard deviations. Independent sample t-tests examined differences between PwPD and caregivers for continuous variables. A chisquare analysis explored differences for categorical variables. A p-value $<0.05$ indicates statistical significance.

\section{Data Integration}

Acceptance of digital health was analyzed by assessing current technology use and purpose, as well as digital health usage from questionnaires and through themes coded from qualitative interviews. Phrases/sentences related to acceptance were categorized as Accept, Neutral, or Reject. To calculate acceptance rate among qualitative interviews, the number of phrases coded as Accept, Neutral, or Reject were counted and 
totaled. The total number of phrases coded as Accept were divided by the total number of phrases coded across the three acceptance categories to calculate acceptance rates among each dyad. The percentages were averaged to calculate an Average Dyadic Acceptance Rate $(n=20)$. Codes from the qualitative interviews were transformed into variables and reported as percent and frequencies. Side-by-side table displays of frequencies derived from perceptions and acceptance (qualitative data) and digital competence scores (quantitative data) were created and interpreted to better describe the population and readiness for a digital health intervention. A Pearson correlation was used to explore if there was an association between the percentage of phrases coded as Hard to Use and the Average Dyadic Acceptance Rate.

\section{Results}

Participant demographics are highlighted in Table 1. All participants identified as Caucasian and one PwPD identified as Hispanic. The majority of caregivers $(85 \%)$ were spouses/partners, while two caregivers were children of PwPD and one was a friend; $80 \%$ of dyads lived together.

\section{Acceptance of Digital Health}

All dyads had access to a laptop or desktop computer and internet connection at home. The majority of participants ( $n=17$ PD and 19 CG) owned a smart phone and $60 \%$ ( $n=11$ PD and 13 caregivers) owned a tablet. Five dyads reported owning an Alexa. One home owned an Amazon Firestick, another had a smart TV, and another reported using a DVR to record shows 
regularly. Most participants (65\%) did not own a smart watch (Apple Watch or FitBit), while $17.5 \%$ reported owning a smart watch but stopped using it and the remaining $17.5 \%$ were currently using a smart watch.

The reasons dyads used technology and the internet are provided in Tables $2 \mathrm{a}$ and $2 \mathrm{~b}$. Twenty-five (62.5\%) participants used the internet for at least five or more health related purposes such as looking for health information for themselves or someone else, looking for information to manage $\mathrm{PD}$, and discussing health concerns with friends/family. Five participants reported not using any technology or do not use the internet for health-related purposes.

There were 466 phrases/sentences coded from the interviews related to Acceptance of digital health and $52.1 \%$ of the phrases were coded as Accept. While $23.4 \%$ of phrases were coded as Neutral and $24.5 \%$ of phrases were coded as Reject. Phrases coded as Accept described the following: the various ways participants used technology in their everyday lives, how they used the internet to find information for managing PD, and/or participants' interest in trying a form of digital health to manage health. The majority of participants reported going to the internet first to look up heath-related questions, especially for managing PD issues. Participants reported using digital health technologies such as patient portals, automated blood pressure cuffs, glucose meters, webinars, and apps to manage diet (e.g. Lose It and the Weight Watchers App) and track steps. Several participants reported setting alarms on their phone as reminders to take their medications 
PD02: "We've done the Weight Watchers app, which is very helpful...you can scan a product's label and it tells you how many points per serving"

Those participants who had a high acceptance of technology reported how their enjoyment for using technology contributed to their desire to try digital health for managing nutrition and/or PD. For instance, when asked about what digital health products they would be interested in for managing food and eating, PD06 stated, "I am the type, if it is digital, I try it." When asked what makes technology and digital health useful, CG05 explained, "I enjoy using it, if you enjoy something you will use it, you can get all that information from so many resources there, I like apps"

Phrases and responses to questions were coded as Neutral when dyads expressed moderate interest for using digital health or specific digital health mediums. For instance, when asked if they would like to try a certain digital health medium and why, some participants were only interested in trying the product if it would benefit their provider. Other participants were interested in trying some products, such as dietary applications and wearable devices, but predicted they would likely lose interest in these mediums over time. For example, when asked if interested in using a Bite Counter, a watch that tracks motion to count bites and estimate calories consumed, PD05 stated:

"If you could tell me that the results would be useful to you, then I would do it as a personal favor since you drove all the way down here!" 
When another dyad was asked if they would be interested in taking pictures of meals and snacks for a nutrition professional to review, a caregiver responded:

CG17: "If nutrition was an issue there might be a reason to do it... if the doctor recommended it."

Other participants said they felt they did not need certain digital health mediums at the moment but may want to take advantage of them in the future as PD progressed. For example, when asked if interested in using a wearable device to monitor gait changes, PD08 stated:

"I'm not at the place where I need that yet, l'd imagine down the road, maybe."

Finally, some participants discontinued their use of digital health mediums, such as wearable devices and dietary tracking apps. One participant stopped using FitBit (a wearable technology device that measures personal health data) due to physical limitations unrelated to PD, which decreased their ability to walk and no longer had many steps to track. This participant reflected,

PD14: "Well when I first got my FitBit and I was kicking out 10,000 a day, and I kept getting all these messages about how good [I'm] doing..."

Another participant stopped using his FitBit because it did not have enough technological features.

PD12: "I just stopped using [FitBit] after a while ...it didn't have enough features, but I mean I did like that it kept track of how often I went up and down the stairs..." 
Phrases that were coded as Reject were typically due to certain digital health mediums or devices. However, some phrases coded were related to a rejection or skepticism of technology in general. For example, when asked if they were interested in using MyFitnessPal, an app to track dietary intake, one dyad stated:

PD09: "I don't think I'm at the risk of eating too much or eating the wrong things."

CG09: "I'm just not interested in knowing that much detail"

When asked how they would like to receive health information, several participants reported preferring hardcopies of literature rather than information provided digitally.

PD07: "I like reading the information, so rather than email or electronic form, I like to see a paper with the information on it. That way I can reference it any time I want."

\section{Perceptions of Digital Health}

There were 189 phrases/sentences coded across the 20 dyadic interviews related to perceptions of digital health. Phrases related to perceptions were categorized as Perceived Usefulness, Perceived Ease of Use, and Awareness of Digital Health. Frequencies of phrases/sentences coded are summarized in Table 3.

Perceived Usefulness. When examining dyadic data related to perceived usefulness, $50 \%$ of dyadic interviews mentioned digital health or technology as Useful. Many of these participants noted that technological advancement could help them not only manage PD but maintain their independence. 
PD06: "I think I will be able to stay driving until the day I die because of autonomous cars. I have no problem with it. I think we are very lucky for the age we are that it is happening now."

Other participants reported finding certain digital health technologies useful specifically for managing diet. For instance, when debating the usefulness of MyFitnessPal with her spouse, a caregiver stated:

CG04: "We need to be more cognizant of the caloric intake because it affects how much you weigh, and if you lost 15 pounds, your core would be much more manageable."

Many participants, $50 \%$ ( $n=10 \mathrm{PwPD}, \mathrm{n}=10$ caregivers) felt that it would be helpful to work with a nutrition professional to manage eating for PD.

PD16: "Well I think it's always good to have access to [a dietitian] that you can ask questions to, but I don't know how much they would be able to do for Parkinson's."

Phrases were coded as Neutral for Perceived Usefulness when participants reported mixed feelings about the benefits of technology or if they were unsure if nutrition services could benefit PD. Supporting quotes from caregivers and PwPD are summarized below.

CG17: "To me a computer is a tool... and l'm not going to sit in front of a screen, when I have other things to do."

PD10: I just type it in and whatever comes up I skim through, and some of it seems valuable and reliable, and some of it seems like a marketing scheme"

Phrases that were coded as Useless when participants reported seeing little value or benefit from using technology. These participants may have also found nutrition interventions to be useless. For example:

PD04: "Some of [technology] is very useful but the majority of it is junk." CG20: "The information that came from [FitBit] was useless" 
In addition to questions around technology, during interviews dyads were also asked to rate how important they felt it was to follow an eating plan for PD. Forty-five percent of dyads agreed that it was important $(n=10 P w P D$, $n=8$ caregivers) to follow a healthy eating plan to manage PD, while $35 \%$ ( $n=6 P w P D, n=8$ caregivers) reported: they were unsure, were neutral, or felt the question was not-applicable because they had not thought about the importance of healthy eating for PD.

Perceived Ease of Use. When examining Perceived Ease of Use among participants, $70 \%$ of interviews contained phrases that were coded as Easy to Use, while $95 \%$ of interviews had phrases coded as Hard to Use. For those phrases/sentences coded as Easy to Use, participants often stated how technology helps them easily access health information. When asked if there was anything that prevents them from learning how to use a technological device or the internet, CG20 responded, "No, it makes life easier." Participants described certain mediums as easier to use than others to access nutrition and health information. For instance, many found email and videoconferencing as an easy medium to receive nutrition information, and several felt that taking pictures of their meals to be reviewed by a dietitian would take little effortful and would be helpful.

CG13: "I can certainly check an email easily. That's probably the simplest, easiest way to get information"

PD11: "I think it's easier to make an appointment, you have more flexibility through a skype session." 
Phrases/sentences were coded as Neutral Ease of Use when participants perceived digital health and technologies as neither difficult nor hard to use. Phases/sentences were also coded as Neutral Ease of Use when participants that were actively using technological devices but reported some annoyances or inconvenience when using the device. However, if these inconveniences did not deter participants from using the device or technological medium, the related phrase or sentence was also coded as Neutral Ease of Use. For instance, one participant summarized her experience with ordering her mealdelivery subscription online:

PD19: "[Sun Basket's] a little bit time consuming, when [on the website], I feel I need time to go through it all, but I do it and it's fine"

Phrases/sentences related to accessing nutrition and health information were also often coded as Neutral Ease of Use. Many participants either had not thought to look for nutrition information specifically for PD or felt that finding nutrition information was easy, but interpreting this information was a challenge. For instance, when asked how easy or difficult is it to find information related to healthy eating, 55\% ( $n=11 P w P D, 11 C G)$, said it was difficult or somewhat difficult. While $17.5 \%$ of participants responded neutral or felt the question was not applicable, with the rationale that they did not know nutrition was important or had not been looking for nutrition information prior to this study.

CG01:"[t's] easy to find, difficult to follow."

CG13: "I would say we haven't really looked for it yet." 
Phrases/sentences were coded as Hard to Use when participants expressed difficulty with using technology. Most phrases coded as Hard to Use were stated by the PwPD.

PD09: "It's [technology] become more complex I think, that bothers me too. I want it to be simpler like it used to be. it's just become more complex and I just don't I know how to do things now."

PD11: “... the cognitive limitations and challenges that come with Parkinson's, you know you can't always read something and immediately translate it into what it is you're supposed to be doing... so sometimes that's frustrating because if you don't understand it you aren't going to use it."

Some participants specifically stated understanding nutrition information could be a challenge and may impact their experience utilizing digital health to manage nutrition,

CG12: "...I feel that nutrition is a particularly difficult topic because [there's] so much conflicting information out there."

Awareness of Digital Health. Phases/sentences related to the theme Awareness of Digital Health were coded as Aware, Somewhat Aware and Not Aware. Phrases/sentences that showed dyads understood what digital health was, were coded as Aware. For instance, CG07 defined digital health as,

"I guess it would be ... a broad term for categories that would have to do with your health and using technology to manage, look up information, to maintain your health, monitor your health."

Phrases/sentences that revealed a limited understanding of digital health or provided an incomplete definition of digital health were coded as Neutral. For instance, PD04, defined digital health as, "I have no idea other than going online and getting some information, but I don't see that as being a useful tool." Phrases/sentences indicating participants did know what digital health 
was were coded as Not Aware. For example, PD12 stated the term digital health "means not being married to your device continuously all day long."

\section{Digital Competence.}

There was no difference in total digital competence scores among PwPD and caregivers, the mean score translates to about a $63.6 \%$ competence level (Table 1). Responses to individual questions form the digital competence questionnaire are summarized in Table 4; the majority of participants (>80\%) felt comfortable finding information, reading or downloading news, and seeking health information. All caregivers and all but two PwPD felt comfortable sending emails. Most participants also felt comfortable buying goods online and internet banking. Participants may need some assistance with using social media and uploading self-created content. About half of the participants may need assistance with completing a videocall.

\section{Data Integration}

The Average Dyadic Acceptance Rate calculated from dyadic interviews was $54.4 \%$. A side-by-side display of individual digital competence scores among PwPD and caregivers, the mean acceptance rate, and percent of phrases coded as Hard to Use were compared (Table 5). Overall, it appears that dyads with higher digital competence scores had higher acceptance rates for technology. However, these acceptance rates could be influenced by the fact that in several dyads, one person was much more comfortable using technology than the other. For instance, within Dyad 01, the PwPD had a 
much higher digital competence score compared to their caregiver $(62.2 \% \mathrm{v}$. $17.8 \%$ ), which may help explain an acceptance rate of $58 \%$ and $50 \%$ of

phrases being coded as Hard to Use. Whereas within Dyad 05, both PwPD and the caregiver had high digital competence scores $(91.1 \%$ and $97.8 \%$ respectively), and an acceptance rate of $80 \%$. During this interview, $50 \%$ of phrases coded as hard to use. There was a negative, significant association between the number of phrases coded as Hard to Use and the Average Dyadic Acceptance Rate $(r=-0.522, p=0.018)$.

\section{Discussion}

This is the first study to analyze the perceptions and acceptance of digital health and digital competence among PwPD and their informal caregivers. It is also the first study aimed to obtain this data to help design a digital nutrition service for dyads. Findings from our study show, the majority of PwPD and their caregivers are currently using technology and have access to technological devices that can be equipped with digital health apps to facilitate delivery of nutrition services. Dyads find technology and digital health mediums useful, but hard to use. Digital competence scores and responses to individual questions provide insight to aspects of technology where PwPD and caregivers may need education and support. Digital health may be a viable medium to increase access to nutrition information related to managing PD, but the added benefits of these services must be clearly communicated to participants. 
Acceptance. An average dyadic acceptance rate of $54.4 \%$ calculated from qualitative phrases coded reveals dyads were interested in specific aspects of technology for assisting with managing PD and nutrition, but also disinterested in technological mediums where they did not see the added benefit. For instance, many dyads were not interested in tracking food or steps or using wearable devices, as they were not interested in knowing that much detail about their health. However, many dyads expressed interest in videoconferencing with a dietitian, receiving nutrition email updates or taking photos of their food to be reviewed by a dietitian. Participants were using technology to email, search the internet, pay bills, and shop online. Over $55 \%$ of dyads used social media and participated in videoconference and watched videos, indicating these tools may be viable mediums to bring nutrition into the home. These findings show that a convenient, user-friendly digital health intervention that provides tailored nutrition information could be a way to improve access to care for this population.

Findings from our study build upon previous research examining the acceptance of technology and digital health among PwPD and their caregivers. Past research has found PwPD and caregivers are interested in using digital health for managing PD (Dorsey et al, 2016; Schulz et al, 2016). A recruitment webpage for a US-based randomized control trial utilizing virtual house calls for PwPD received 11,000 individual views worldwide (Dorsey et al, 2016). Ozanne et al (2017) found that PwPD saw the potential for wearable devices to improve treatment and felt this benefit outweighed the 
inconvenience of having to wear a sensor. A study completed by Duroseau and colleagues (2016) examined acceptance among PwPD for using multiple electronic mediums to receive instructions and communicate with healthcare providers and found that older PwPD had a less favorable view of using technology to learn about their care plan and communicate with healthcare providers. Our studies expand upon previous research by specifically examining the acceptance of these technological mediums to receive nutrition information and interact with nutrition experts and includes the opinion of caregivers.

Perceptions. Findings from qualitative analyses reveal that dyads perceive technology and digital health to be useful, but hard to use. Interviews revealed many of our participants, were also not aware of what the term digital health meant. These perceptions expand upon previous research examining views of PwPD around digital health. In a study completed by Duroseau et al (2016), nearly 65\% of PwPD reported they were willing to use electronic methods and $48 \%$ believed using technology to communicate with providers would help to better understand their care (Duroseau et al, 2016). When stratifying participants by age, those 65 and over were less likely to believe using technology to communicate with a healthcare provider would enhance their understanding of care; this is attributed to the fact that older patients may not be as comfortable with using technology (Duroseau et al, 2016). This research, as well as our current study, indicates optimal communication 
mediums among PwPD may vary based on patient demographics and that training older PwPD to use technology may be warranted.

Digital Competence. Findings support the need for educational training of digital health mediums among PwPD and their informal caregivers before implementing a digital health intervention. The reported reasons dyads were using technology matched their responses to the individual questions on the digital competence questionnaire. For instance, most participants are comfortable with corresponding via email, searching for health information and services, and shopping online. However, installing new devices and using social networks may be problematic for some participants. To help increase competence and perceived ease of use, future research could look to models such as Cyber Seniors ${ }^{\circledR}$, an intergenerational program where college and high school students help older adults learn about technology (Rusnack \& Cassady, 2014; Leedahl et al, 2018). Additionally, more information is needed about PwPD's and caregivers' knowledge of nutrition for managing PD and health literacy to ensure this population is accessing accurate and reliable nutrition information.

Data Integration. There was a negative, significant association between the number of phrases coded as Hard to Use and the Average Dyadic Acceptance Rate. This relationship combined with the lack of awareness of digital health among dyads and how nutrition can help manage PD, may help explain why dyads rejected certain digital health mediums. Finally, within dyads, digital competence scores varied, with one person within 
the patient-caregiver dyad having a higher digital competence score than the other. This may account for the acceptance rate falling just above $50 \%$. Future research should consider exploring ways to increase the acceptance rate among PwPD and caregivers, as well as educating dyads about how digital health can help enhance disease management. Research should also explore the facilitators and barriers for digital health adoption among dyads.

Strengths and Limitations. Our study design promotes patient- and caregiver-centered care for managing PD and supports both as part of the healthcare team. Additionally, the utilization of mixed-methods study design provides an in-depth understanding of dyads' perception, acceptance and current level of digital competence. The use of semi-structured dyadic interviews is a strength, as dyadic interviews promote interaction between participants to help provide detailed information with regard to their experience on the topic of interest (Morgan, Eliot, Lowe, \& Gorman, 2016). As a result, findings from this study can be incorporated in the TAM (Davis, Bagozzi, \& Warshaw, 1989) and the emerging middle range theories of transition (Meleis et al, 2000).

While this study is novel it is not without limitations. The majority of participants were educated, Caucasian and had access to technology. Additionally, our participants were all from the northeast region of the United States. As a result, findings may not be generalizable to PwPD in other regions of the country or from marginalized populations. Additionally, since cognitive status was an inclusion criterion to enroll in our study, many of our 
participants did not fully rely on caregivers and were able to perform many day-to-day activities on their own. However, including caregivers early at disease diagnosis and encouraging caregivers to partner with healthcare providers can help reduce stress and family conflict (Roth, Fredman, \& Haley, 2015). Another limitation of this study is that the digital competence survey is not a validated tool, however items were adopted from the European Union Wide Indicators of Digital Competence, which is seen in the literature to create a digital competence framework (Görgényi Hegyes, Csapó, \& Fekete Farkas, 2017). Future research should explore validating this instrument among both the general population and the PD community. Additionally, similar survey questions around technology acceptance have been used to survey PwPD in previous research (Duroseau et al, 2016). A final study limitation is that disease stage was only obtained from some of the PwPD. Requests were sent to physician offices for personal health information, but we were not able to obtain this information for all 20 PwPD.

Implications. Results from this study can be used to help design and implement an acceptable digital health service to assist PwPD and caregivers manage nutrition. For this service to be accepted among dyads the benefits of utilizing technology and healthy eating must be clearly communicated to endusers. Training of the digital health service must be provided prior to implementing an intervention. Experts suggest when designing digital health interventions specifically for PwPD, developers should consider both PwPD and caregiver views, needs and preferences (Espay et al., 2019). Our findings 
support recommendations presented by Duroseau and colleagues (2016), who suggest services be tailored to meet the technological preferences of PwPD and exploration of these preferences through qualitative research. These remote services can help increase access to nutrition information among PwPD and caregivers and have the potential to improve health-related quality of life, disease and caregiver burden.

Conclusion. This mixed-methods study focused on describing the acceptance and perceptions of digital health to manage nutrition for PwPD and their caregivers, as well as describe their level of digital competence. Results indicate mixed acceptance rates for technology and digital health mediums among dyads, possibly due to many participants perceiving digital health as useful, but hard to use. Digital competence scores suggest dyads participating in a digital health nutrition intervention will need some training prior to study participation. Findings from this study complement existing literature regarding digital health for managing PD and helps to better understand the opportunity to use digital health as an avenue to include nutrition and caregivers in the PD care plan. Future studies should explore digital health and technology as tools to provide evidenced-based nutrition and health knowledge to PwPD and caregivers. Prior to launching a digital health service to manage nutrition, dyads will need training and technical support. 


\section{Chapter 3 Tables and Figures}

Table 1: Participant Demographics

\begin{tabular}{|c|c|c|c|}
\hline Descriptive Variables & PwPD $(n=20)$ & Caregivers $(n=20)$ & Range \\
\hline Age (years) & $69.7 \pm 9.2$ & $66.4 \pm 13.0$ & $39-89$ \\
\hline $\begin{array}{l}\text { Gender } \mathrm{n}(\%) \\
\text { Male } \\
\text { Female }\end{array}$ & $\begin{array}{c}13(65) \\
7(35)\end{array}$ & $\begin{array}{c}4(20) \\
16(80)\end{array}$ & NA \\
\hline $\begin{array}{l}\text { Education } \mathrm{n}(\%) \\
\text { - } \text { HS Diploma/Some College } \\
\text { - Technical Training/Trade } \\
\text { School/Associates } \\
\text { - } \geq \text { College Degree or Greater }\end{array}$ & $\begin{array}{l}6(30) \\
3(15) \\
11(55)\end{array}$ & $\begin{array}{c}1(5) \\
5(25) \\
14(70)\end{array}$ & NA \\
\hline $\begin{array}{cl}\text { Employment Status } \mathrm{n}(\%) \\
\text { - Retired } \\
\text { - } \\
\text { - } & \text { Fart Time Time }\end{array}$ & $\begin{array}{l}15(75) \\
3(15) \\
2(10)\end{array}$ & $\begin{array}{l}10(50) \\
2(10) \\
8(40)\end{array}$ & NA \\
\hline T-MoCA & $19.8 \pm 1.5$ & $20.4 \pm 1.1$ & $18-22$ \\
\hline Years Since Diagnosis & $7.6 \pm 5.4$ & NA & $0.33-18.0$ \\
\hline Disease Burden (PDQ-39) & $21.7 \pm 3.5$ & NA & $3.12-50.01$ \\
\hline Caregiver Burden (MCSI) ${ }^{\dagger}$ & NA & $12.6 \pm 8.2$ & $0-26$ \\
\hline $\mathrm{BMI}\left(\mathrm{kg} / \mathrm{m}^{2}\right)$ & $27.09 \pm 5.4$ & $28.3 \pm 5.7$ & $15.4-43.1$ \\
\hline DST Scores & $56.95 \pm 9.3$ & $59.5 \pm 10.7$ & $37-81$ \\
\hline $\begin{array}{c}\text { Nutrition Risk n(\%) } \\
\text { - At Risk } \\
\text { - Possible Risk } \\
\text { - } \text { Not At Risk }\end{array}$ & $\begin{array}{c}10(50) \\
10(50) \\
0(0)\end{array}$ & $\begin{array}{c}11(55) \\
8(40) \\
1(5)\end{array}$ & NA \\
\hline Digital Competence & $27.5 \pm 12.8$ & $29.7 \pm 12.6$ & $0-45$ \\
\hline
\end{tabular}

Data reported as $\mathrm{n}(\%)$ for categorical variables and mean \pm sd for continuous variables; ${ }^{\dagger} \mathrm{n}=19$; Abbreviations: $\mathrm{HS}=$ high school, T-MoCA=Telephone Montreal Cognitive Assessment, $\mathrm{BMI}=$ body mass index, DST=dietary screening tool. 
Table 2a: Distribution of Technological Purposes Among PwPD and Caregivers Reported in Frequencies and Percentages

\begin{tabular}{|l|c|c|}
\hline Variables n(\%) & $\begin{array}{c}\text { PwPD } \\
\text { (n=20) }\end{array}$ & $\begin{array}{c}\text { Caregivers } \\
\text { (n=20) }\end{array}$ \\
\hline Email & $18(90)$ & $20(100)$ \\
\hline Social Media & $10(50)$ & $12(60)$ \\
\hline Videos (Youtube) & $13(65)$ & $14(70)$ \\
\hline Video Conference & $11(55)$ & $8(40)$ \\
\hline Search Internet & $17(85)$ & $17(85)$ \\
\hline E-banking/paying bills & $14(70)$ & $17(85)$ \\
\hline E-Shopping & $15(75)$ & $16(80)$ \\
\hline
\end{tabular}

Table 2b: Description of Internet Use for managing Health Among PwPD and Caregivers Reported in Frequencies and Percentages

\begin{tabular}{|l|c|c|c|}
\hline Variables n(\%) & $\begin{array}{c}\text { PwPD } \\
\text { (n=20) }\end{array}$ & $\begin{array}{c}\text { Caregivers } \\
\text { (n=20) }\end{array}$ & phi \\
\hline $\begin{array}{l}\text { Look for health or medical information for } \\
\text { yourself }\end{array}$ & $15(75)$ & $18(90)$ & 0.197 \\
\hline $\begin{array}{l}\text { Looked for health or medical information for } \\
\text { someone else }\end{array}$ & $11(55)$ & $17(85)$ & 0.327 \\
\hline $\begin{array}{l}\text { Looked for information about managing } \\
\text { Parkinson's disease }\end{array}$ & $13(65)$ & $14(70)$ & 0.053 \\
\hline $\begin{array}{l}\text { Participated in online forums or support } \\
\text { groups for people with similar health or } \\
\text { medical issue }\end{array}$ & $1(5)$ & $4(20)$ & 0.227 \\
\hline $\begin{array}{l}\text { Used a website to help you with your diet, } \\
\text { weight or physical activity }\end{array}$ & $7(35)$ & $8(40)$ & 0.052 \\
\hline Looked for a healthcare provider & $7(35)$ & $5(25)$ & -0.109 \\
\hline $\begin{array}{l}\text { Downloaded health information to a mobile } \\
\text { device, such as an MP3 player, cell phone, } \\
\text { tablet computer, or electronic book device }\end{array}$ & $8(40)$ & $4(20)$ & -0.218 \\
\hline $\begin{array}{l}\text { Shared health information on social media } \\
\text { sites (Facebook, Twitter) }\end{array}$ & $1(5)$ & $11(55)$ & $12(60)$ \\
\hline $\begin{array}{l}\text { Exchanged support about health concerns } \\
\text { with family and friends }\end{array}$ & $12(60)$ & $12(60)$ & 0.051 \\
\hline $\begin{array}{l}\text { Kept track of personal health information } \\
\text { such as care received, test results, or } \\
\text { upcoming medical appointments }\end{array}$ & $10(50)$ & $6(30)$ & -0.204 \\
\hline Watched a health-related video on YouTube & & \\
\hline
\end{tabular}

Values are reported as $\mathrm{n}(\%)$. Chi Square Completed (looked for health info for someone else phi=0.327, $p=0.008)$ Effect size reported as phi ( 0.1 = small; $0.30=$ medium; $0.5=$ large $)$. 
Table 3: Themes for Acceptance and Perception of Digital Health Summarized by Number of Phrases Coded, Percent of Comments, and Number of Dyads Mentioning Acceptance or Perception within Each Category

\begin{tabular}{|c|c|c|c|}
\hline & $\begin{array}{c}\text { Number of } \\
\text { Phrases } \\
\text { Coded }\end{array}$ & $\begin{array}{c}\text { Percent of } \\
\text { Comments }\end{array}$ & $\begin{array}{c}\text { Number of Dyads } \\
\text { Mentioning } \\
\text { Code/Theme Within } \\
\text { Each Category }\end{array}$ \\
\hline Acceptance & 466 & - & 20 \\
\hline Accept & 243 & $54.4 \%$ & 20 \\
\hline Neutral & 109 & $23.4 \%$ & 20 \\
\hline Reject & 114 & $24.5 \%$ & 19 \\
\hline Perceived Usefulness & 29 & - & 11 \\
\hline Useful & 22 & $75.9 \%$ & 10 \\
\hline Neutral & 4 & $13.8 \%$ & 4 \\
\hline Useless & 3 & $10.3 \%$ & 3 \\
\hline Perceived Ease of Use & 104 & - & 20 \\
\hline Easy to Use & 22 & $21.2 \%$ & 14 \\
\hline Neutral Ease of Use & 12 & $11.5 \%$ & 10 \\
\hline Hard to Use & 70 & $67.3 \%$ & 19 \\
\hline Awareness of Digital Health & 56 & - & 20 \\
\hline Aware & 11 & $19.6 \%$ & 8 \\
\hline Neutral Awareness & 17 & $30.4 \%$ & 13 \\
\hline Not Aware & 28 & $50 \%$ & 16 \\
\hline
\end{tabular}


Table 4: Those Participants Who Responded Slightly or Strongly Agree to Individual Digital Competence Questions Among PwPD and Caregivers Reported by Frequency (Percentage)

\begin{tabular}{|l|c|c|c|}
\hline Digital Competence Question n(\%) & $\begin{array}{c}\text { PwPD } \\
\text { (n=20) }\end{array}$ & $\begin{array}{c}\text { Caregivers } \\
\text { (n=20) }\end{array}$ & Phi \\
\hline $\begin{array}{l}\text { Searching and finding information about goods and } \\
\text { services }\end{array}$ & $18(90)$ & $18(90)$ & 0.0 \\
\hline $\begin{array}{l}\text { Reading or downloading news/newspapers/news } \\
\text { magazines }\end{array}$ & $16(80)$ & $17(85)$ & 0.07 \\
\hline Using copy/paste tools & $13(65)$ & $13(65)$ & 0.0 \\
\hline Seeking health information & $17(85)$ & $17(85)$ & 0.0 \\
\hline Sending/receiving emails & $18(90)$ & $20(100)$ & 0.23 \\
\hline Using videocalls, such as skype & $11(55)$ & $10(50)$ & -0.05 \\
\hline Participating in social networks & $11(55)$ & $12(60)$ & 0.05 \\
\hline Posting messages on social networks & $9(45)$ & $12(60)$ & 0.15 \\
\hline $\begin{array}{l}\text { Uploading self-created content to any website to be } \\
\text { shared }\end{array}$ & $7(35)$ & $7(35)$ & 0.0 \\
\hline Sharing talents and ideas with on social networks & $6(30)$ & $9(45)$ & 0.16 \\
\hline Sharing interests and ideas with those you know & $13(65)$ & $16(80)$ & 0.17 \\
\hline Connecting and installing new devices & $12(60)$ & $12(60)$ & 0.0 \\
\hline Internet banking & $13(65)$ & $14(70)$ & 0.05 \\
\hline $\begin{array}{l}\text { Buying or ordering goods or services for private use } \\
\text { (last 12 months) over the internet }\end{array}$ & $16(80)$ & $15(75)$ & -0.06 \\
\hline Making an appointment with a practitioner via a website & $12(60)$ & $14(70)$ & 0.105 \\
\hline
\end{tabular}

Chi Square Analyses Completed to compare between group differences; phi co-efficient used to report effect-size: small $=0.01$, medium $=0.30$, larger $=0.50$ 
Table 5: Data Integration: Side by Side Display of Digital Competence Scores (total scores(\%)), Acceptance Rates Calculated From Semi-Structured Interviews

\begin{tabular}{|c|c|c|c|c|}
\hline Dyads & $\begin{array}{l}\text { PwPD Digital } \\
\text { Competence }\end{array}$ & $\begin{array}{l}\text { Caregiver Digital } \\
\text { Competence }\end{array}$ & $\begin{array}{c}\text { Acceptance Rate } \\
(\%)\end{array}$ & $\begin{array}{c}\text { Hard to Use } \\
\text { (\% phrases } \\
\text { coded) }\end{array}$ \\
\hline Dyad 1 & $28(62.2)$ & $8(17.8)$ & 58.3 & 50.0 \\
\hline Dyad 2 & $33(73.3)$ & $26(57.8)$ & 90.0 & 25.0 \\
\hline Dyad 3 & $34(75.6)$ & $25(55.6)$ & 80 & 0.0 \\
\hline Dyad 4 & $18(40)$ & $31(68.9)$ & 31.6 & 60.0 \\
\hline Dyad 5 & 41 (91.1) & $44(97.8)$ & 80.0 & 50.0 \\
\hline Dyad 6 & 41 (91.1) & $33(73.3)$ & 78.9 & 25.0 \\
\hline Dyad 7 & 31 (68.9) & $45(100)$ & 50.0 & 50.0 \\
\hline Dyad 8 & $4(8.9)$ & $25(55.6)$ & 31.8 & 100 \\
\hline Dyad 9 & $25(55.6)$ & $4(8.9)$ & 31.8 & 100 \\
\hline Dyad 10 & $39(86.7)$ & 40 (88.9) & 69.4 & 87.5 \\
\hline Dyad 11 & $40(88.9)$ & $39(86.7)$ & 69.4 & 87.5 \\
\hline Dyad 12 & $45(100)$ & 41(91.1) & 51.7 & 55.6 \\
\hline Dyad 13 & $16(35.6)$ & $29(64.4)$ & 25.0 & 50.0 \\
\hline Dyad 14 & $32(71.1)$ & $7(15.6)$ & 48.1 & 75.0 \\
\hline Dyad 15 & $21(46.7)$ & 31 (68.9) & 39.3 & 80.0 \\
\hline Dyad 16 & $23(51.1)$ & $35(77.8)$ & 48.4 & 50.0 \\
\hline Dyad 17 & $16(35.6)$ & $14(31.1)$ & 31.8 & 75.0 \\
\hline Dyad 18 & $0(0)$ & 42 (93.3) & 44.8 & 83.3 \\
\hline Dyad 19 & $19(42.2)$ & $39(86.7)$ & 57.1 & 100 \\
\hline Dyad 20 & $44(97.8)$ & $36(80)$ & 69.2 & 66.7 \\
\hline
\end{tabular}

Digital Competence scores are reported for both PwPD and Caregivers and are reported as total score(percentage). Acceptance Rate reported which was calculated by dividing phrases coded as Accept by total number of phrases coded related to Accept, Neutral and Reject for each interview. Percent of phrases coded as hard to use in each interview were calculated by dividing phrases coded as hard to use by total phrases coded related to Ease of Use.

\section{References:}

Achey, M., Aldred, J. L., Aljehani, N., Bloem, B. R., Biglan, K. M., Chan, P., . . Movement Disorder Society Telemedicine Task, F. (2014). The past, present, and future of telemedicine for Parkinson's disease. Mov Disord, 29(7), 871883. doi:10.1002/mds. 25903

Attard, A., \& Coulson, N. S. (2012). A thematic analysis of patient communication inParkinson's disease online support group discussion forums. Computers in Human Behavior, 38, 500-506. 
Bailey, R. L., Mitchell, D. C., Miller, C. K., Still, C. D., Jensen, G. L., Tucker, K. L., \& Smiciklas-Wright, H. (2007). A dietary screening questionnaire identifies dietary patterns in older adults. J Nutr., 137, 421-426.

Barichella, M., Cereda, E., \& Pezzoli, G. (2009). Major nutritional issues in the management of Parkinson's disease. Mov Disord, 24(13), 18811892.doi:10.1002/mds.22705.

Bazeley, P., \& Jackson, K. (2013). Qualitative data analysis with NVivo. (2nd edn). Thousand Oaks, CA:Sage.

Boersma, I., Jones, J., Carter, J., Bekelman, D., Miyasaki, J., Kutner, J., \& Kluger, B. (2016). Parkinson disease patients' perspectives on palliative care needs: What are they telling us? Neurol Clin Pract, 6(3), 209-219. doi:10.1212/CPJ.0000000000000233

Borlongan, C. V., Burns, J., Tajiri, N., Stahl, C. E., Weinbren, N. L., Shojo, H., . . . van Loveren, H. R. (2013). Epidemiological survey-based formulae to approximate incidence and prevalence of neurological disorders in the United States: A meta-analysis. PLoS One, 8(10), e78490.

doi:10.1371/journal.pone.0078490

Castanho, T. C., Amorim, L., Zihl, J., Palha, J. A., Sousa, N., \& Santos, N. C. (2014). Telephone-based screening tools for mild cognitive impairment and dementia in aging studies: A review of validated instruments. Front Aging Neurosci, 6, 16. doi:10.3389/fnagi.2014.00016

Creswell, J.W. and Plano Clark, V.L. Designing and Conducting Mixed Methods Research (2018). Third edition. Sage Publications Inc. Thousand Oaks,California

Davis, F. D., Bagozzi, R. P., \& Warshaw, P. R. (1989). User acceptance of computer technology: A comparison of two theoretical models. Manage Sci, 35(8), 982-1003 
Dorsey, E. R., Venkataraman, V., Grana, M. J., Bull, M. T., George, B. P., Boyd, C. M., ... Biglan, K. M. (2013). Randomized controlled clinical trial of "virtual house calls" for Parkinson disease. JAMA Neurol, 70(5), 565-570. doi:10.1001/jamaneurol.2013.123

Dorsey, E. R., Vlaanderen, F. P., Engelen, L. J., Kieburtz, K., Zhu, W., Biglan, K. M., . . Bloem, B. R. (2016). Moving Parkinson care to the home. Mov Disord, 31(9), 1258-1262. doi:10.1002/mds.26744

Ducharme, F. C., Levesque, L. L., Lachance, L. M., Kergoat, M. J., Legault, A. J.,Beaudet, L. M., \& Zarit, S. H. (2011). "Learning to become a family caregiver" efficacy of an intervention program for caregivers following diagnosis of dementia in a relative. Gerontologist, 51(4), 484-494. doi:10.1093/geront/gnr014

Duroseau, N., Abramson, T., Pergament, K., Chan, V., Govindavari, J. P., Ciraco, C., ... Krishnamachari, B. (2017). Acceptance of technology-based tools in a sample of Parkinson's patients. Chronic IIIn, 13(1), 3-13.

doi:10.1177/1742395316653453

Espay, A. J., Bonato, P., Nahab, F. B., Maetzler, W., Dean, J. M., Klucken, J., . . Movement Disorders Society Task Force on, T. (2016). Technology in Parkinson's disease: Challenges and opportunities. Mov Disord, 31(9), 12721282. doi:10.1002/mds.26642

Espay, A. J., Hausdorff, J. M., Sanchez-Ferro, A., Klucken, J., Merola, A., Bonato, P., . . Movement Disorder Society Task Force on, T. (2019). A roadmap for implementation of patient-centered digital outcome measures in Parkinson's disease obtained using mobile health technologies. Mov Disord, 34(5), 657-663. doi:10.1002/mds.27671

European Commission. Measuring digital skills across the EU: EU wide indicators of digital competence. (2014). Retrieved from

https://ec.europa.eu/digital-single-market/en/news/measuring-digital-skillsacross-eu-eu-wide-indicators-digital-competence

Fahn, S. (2003). Description of Parkinson's disease as a clinical syndrome. Ann N Y Acad Sci, 991, 1-14. 
Gale, N. K., Heath, G., Cameron, E., Rashid, S., \& Redwood, S. (2013). Using the framework method for the analysis of qualitative data in multidisciplinary health research. BMC Med Res Methodol, 13, 117. doi:10.1186/1471-2288-13-117

Görgényi Hegyes, E., Csapó, I., Fekete Farkas, M. (2017). Some aspects of digitalization and sustainability in the European Union. Journal of Management, (31)2.

Jenkinson, C., Fitzpatrick, R., Peto, V., Greenhall, R., \& Hyman, N. (1997). The Parkinson's Disease Questionnaire (PDQ-39): development and validation of a Parkinson's disease summary index score. Age Ageing, 26(5), 353-357. Retrieved from http://www.ncbi.nlm.nih.gov/pubmed/9351479

Kontos, E., Blake, K. D., Chou, W. Y., \& Prestin, A. (2014). Predictors of eHealth usage: Insights on the digital divide from the Health Information National Trends Survey 2012. J Med Internet Res, 16(7), e172. doi:10.2196/jmir.3117

Kowal, S. L., Dall, T. M., Chakrabarti, R., Storm, M. V., \& Jain, A. (2013). The current and projected economic burden of Parkinson's disease in the United States. Mov Disorder, 28(3), 311-218.

Leedahl, S. N., Brasher, M. S., Estus, E., Breck, B. M., Dennis, C. B., \& Clark, S. C. (2018). Implementing an interdisciplinary intergenerational program using the Cyber Seniors ${ }^{(R)}$ reverse mentoring model within higher education. Gerontol Geriatr Educ, 1-19. doi:10.1080/02701960.2018.1428574

LoBuono, D. L., Paulin, C., Furong, X., Mahler, L., Delmonico, M. J., \& Lofgren, I. E. (2018). Parkinson's awareness and the role of a health care team in managing Parkinson's disease. The Digest, 53(2), 13-20.

LoBuono, D.L., Shea, K.S., Tovar, A., Leedahl, S.N., Mahler, L., Xu, F., Lofgren, I.E. (In Preparation). Diet Quality and Nutrition Concerns of People with Parkinson's Disease and Their Informal Caregivers: A Mixed Methods Study. JAND. 
Meleis, A. I., Sawyer, L. M., Im, E. O., Hilfinger Messias, D. K., \& Schumacher, K. (2000). Experiencing transitions: An emerging middle-range theory. ANS Adv Nurs Sci, 23(1), 12-28.

Meyer, M., Hartwell, J., Beatty, A., \& Cattell, T. (2019). Creation of a Virtual Nutrition Support Team to Improve Quality of Care for Patients Receiving Parenteral Nutrition in a Multisite Healthcare System. Nutr Clin Pract. doi:10.1002/ncp.10375

National Cancer Institute. Health Information National Trends Survey 4 (HINTS 4): HINTS-FDA Methodologies (2015). Retrieved from https://hints.cancer.gov/docs/Instruments/HINTSFDA Methodology Report.pdf

Nelson, D. E., Kreps, G. L., Hesse, B. W., Croyle, R. T., Willis, G., Arora, N. K., . . Alden, S. (2004). The Health Information National Trends Survey (HINTS): development, design, and dissemination. J Health Commun, 9(5), 443-460; discussion 481-444. doi:10.1080/10810730490504233

Ozanne, A., Johansson, D., Hallgren Graneheim, U., Malmgren, K., Bergquist, F., \& Alt Murphy, M. (2018). Wearables in epilepsy and Parkinson's disease-A focus group study. Acta Neurol Scand, 137(2), 188-194.

doi:10.1111/ane.12798

Parkinson's Australia Inc. Living with Parkinson's Disease: An updated economic analysis 2014 Retrieved from Deloitte Access Economics https://docs.wixstatic.com/ugd/bfe057 b766845ec05b4256bbf5b290baa12139 .pdf

Pendlebury, S. T., Welch, S. J., Cuthbertson, F. C., Mariz, J., Mehta, Z., \& Rothwell, P.M. (2013). Telephone assessment of cognition after transient ischemic attack and stroke: Modified telephone interview of cognitive status and telephone Montreal Cognitive Assessment versus face-to-face Montreal Cognitive Assessment and neuropsychological battery. Stroke, 44(1), 227229. doi:10.1161/STROKEAHA.112.673384

Roth, D.L., Fredman, L., \& Haley, W.E. (2015). Informal caregiving and its impact on health: a reappraisal from population-based studies. Gerontologist, 55(2), 309-319. doi: 10.1093/geront/gnu177 
Rusnack, B. \& Cassady, S. (2014). Cyber-Seniors [DVD]. Canada: The Best Part, Inc.

Saladaña, J. (2016). The coding manual for qualitative researchers. 3nd edn). Thousand Oaks, CA: Sage

Schulz, R., Beach, S. R., Matthews, J. T., Courtney, K., De Vito Dabbs, A., \& Mecca, L.P. (2016). Caregivers' willingness to pay for technologies to support caregiving.Gerontologist, 56(5), 817-829. doi:10.1093/geront/gnv033

Shah, S. P., Glenn, G. L., Hummel, E. M., Hamilton, J. M., Martine, R. R., Duda, J. E., \& Wilkinson, J. R. (2015). Caregiver tele-support group for Parkinson's disease: A pilot study. Geriatr Nurs, 36(3), 207-211.

doi:10.1016/j.gerinurse.2015.02.002

Shosha, G. A. (2012). Employment of Colaizzi's Strategy in Descriptive Phenomenology: A Reflection of a Researcher. European Scientific Journal, 8(27), 31-43.

Siddique, A. B., Krebs, M., Alvarez, S., Greenspan, I., Patel, A., Kinsolving, J., \& Koizumi, N. (2019). Mobile Apps for the Care Management of Chronic Kidney and End-Stage Renal Diseases: Systematic Search in App Stores and Evaluation. JMIR Mhealth Uhealth, 7(9), e12604. doi:10.2196/12604

Stamford, J. A., Schmidt, P. N., \& Friedl, K. E. (2015). What engineering technology could do for quality of life in Parkinson's disease: A review of current needs and opportunities. IEEE J Biomed Health Inform, 19(6), 18621872. doi:10.1109/JBHI.2015.2464354

Stull, D. (1996). The multidimensional caregiver strain index (MCSI): Its measurement and structure. J Clin Geropsychol, 2(11), 175-196.

Thomas, J. G., \& Bond, D. S. (2014). Review of innovations in digital health technology to promote weight control. Curr Diab Rep, 14(5), 485.

doi:10.1007/s11892-014-0485-1 
Ventura Marra, M., Shotwell, M., Nelson, K., \& Malone, J. (2017). Improving weight status in obese middle-aged and older men through telenutrition.

Innovations in Aging, (Suppl 1), 635-636. doi:10.1093/geroni/igx004.2242

Vikdahl, M., Domellof, M. E., Forsgren, L., \& Haglin, L. (2015). Olfactory function, eating ability, and visceral obesity associated with MMSE three years after Parkinson's disease diagnosis. J Nutr Health Aging, 19(9), 894-900. doi:10.1007/s12603-015-0573-1

World Health Organization. WHO Guideline: Recommendations on Digital Interventions for Health System Strengthening. (2019). Geneva: World Health Organization, License: CC BY-NC-SA 3.0 IGO.

Zizzo, N., Bell, E., Lafontaine, A. L., \& Racine, E. (2017). Examining chronic care patient preferences for involvement in health-care decision making: The case of Parkinson's disease patients in a patient-centered clinic. Health Expect, 20(4), 655-664. doi:10.1111/hex.12497 


\section{APPENDIX A: Review of the Literature}

I. What is Parkinson's Disease

Parkinson's disease (PD) is an incurable, progressive neurodegenerative movement disorder that traditionally occurs in the second half of life. ${ }^{1}$ Over 900,000 Americans live with PD, approximately 60,000 new cases are diagnosed each year ${ }^{2,3}$, and the national economic burden of PD exceeds $\$ 14.4$ billion. ${ }^{4}$ The life expectancy from PD onset of diagnosis to death is approximately 15 years. ${ }^{5,6}$ The number of people with PD (PwPD) living in the US is expected to double by 2030 due to the growing number of people over 65 and an increase in life expectancy for PwPD. As a result, researchers and clinicians have recently started investigating symptoms and clinical features of PwPD 20 years out. ${ }^{7}$

The exact etiology of PD remains unknown but it is hypothesized to arise from an interaction between environmental and genetic factors resulting in degeneration of neurons. ${ }^{8}$ Parkinson's disease results when there is a disruption of dopaminergic neurotransmission within the basal ganglia of the brain. ${ }^{9}$ Dopamine are neurotransmitters that control motor function and movement control, as well as reward-motivated beahavior. ${ }^{9}$ As a result of PD, dopaminergic neurons in the substantia nigra are decreased. Within the residual dopaminergic neurons, Lewy bodies (deposits of alpha-synuclein) present. ${ }^{9}$ The basal ganglia, located in the forebrain, controls voluntary movement, procedural and habitual learning, eye movement, cognition and emotion..$^{10}$ The substantia nigra, part of the basal ganglia circuitry, located in 
the mid-brain, plays a role in movement and reward. ${ }^{10}$ As PD progresses, problems extend beyond motor deficits and can impact nutrition, weight management, cognitive and physical functioning.

The diagnosis and therefore the prevalence and incidence of PD varies by age, geographic location, race, and gender. ${ }^{11}$ Prevalence of PD increases with age, with the age of onset around 60 years old, ${ }^{8}$ and impacting $2.6 \%$ of Americans who are 85-89 years of age ${ }^{8}$ Parkinson's disease infrequently occurs under the age of 40 years old and early onset increases the probability genetics may play role. ${ }^{12}$ Rates of PD are highest in the Midwest and Northeast regions of the United States (US), with rates being up to 10 times higher than rates in the Western and Southern regions of the US. ${ }^{11}$ There is a higher occurrence of PD among males, with a male to female ratio of $3: 2 .{ }^{12,13}$ Whether or not PD is more prevalent among whites versus non-whites needs further exploration. ${ }^{11}$ Research regarding the prevalence of PD across race and ethnicity is consistent and inconclusive..$^{11,14}$

Parkinson's disease can be characterized as idiopathic PD (primary parkinsonism) or non-idiopathic PD (secondary or atypical parkinsonism).${ }^{9}$ It is estimated that up to $85 \%$ are diagnosed with idiopathic $\mathrm{PD}^{15}$ and respond well to dopaminergic medications. Those with non-idiopathic PD do not respond well to dopaminergic medications. ${ }^{9}$ Idiopathic PD can be characterized into two main subtypes: tremor dominant (TD) and postural instability gait difficulty (PIGD). ${ }^{16}$ 
Currently there is no cure for PD. Levodopa, a medication that produces dopamine in the central nervous system, is the most common medication used to control motor sequelae. ${ }^{17,18}$ Levodopa is cost-effective ${ }^{14}$ and considered the most efficacious treatment because it improves motor function, quality of life (QOL), and reduces morbidity and mortality. ${ }^{15} \mathrm{An}$ "on" state is when motor symptoms subside when levodopa is taken, and an "off state" is where levodopa wears off before the next dose and PD motor symptoms are present. Off periods can result in functional disability and can be characterized by stiffness, slowness, tremor, as well as cognitive and mood changes. ${ }^{19}$ The negatives of levodopa treatment are: 1) causes motorsymptoms which impact HRQOL and ability to perform activities of daily living (ADLs); and 2) over-time, patients build up a tolerance to levodopa and may require higher levodopa doses and eventually the medication loses effectiveness. ${ }^{20}$

About 6.5 million informal caregivers provide substantial help for medical and dietary management for older adults living with disability. ${ }^{21}$ Informal caregivers are any unpaid family member or friend who provides the majority of care. ${ }^{22}$ Almost $55 \%$ of caregivers for older adults assist with medication management ${ }^{23}$, and caregivers of PwPD likely play an integral role in managing food-drug interactions, given their role in managing dietary intake for PwPD. ${ }^{24}$ With an increase in life expectancy for PwPD but no cure for PD, the socioeconomic and personal burdens for PwPD and their informal caregivers will also rise, which includes an exponential increase in excess 
medical spending..$^{4,12,25}$ As the disease progresses, informal caregiver responsibility increases and caregivers will be play a central role in utilizing digital health to access care for their loved one with PD. ${ }^{26}$

In general, caregivers spend $24^{+}$hours per week caring for their loved one ${ }^{22}$, but caregivers of PwPD spend up to 40 hours per week performing care-related duties ${ }^{27}$ Compared to the general population, caregivers for PwPD are more likely to have depression, anxiety, decreased health status ${ }^{28}$, and/or poorer $\mathrm{QOL}^{29}$, which is partly attributed to observing the physical and cognitive decline of their loved one with $\mathrm{PD}^{29}$. Caregiver stress and burden can adversely affect caregiver's psychosocial and physical functioning, which may compromise the care provided and the health of the patient-caregiver dyad. ${ }^{28,30}$. Specific to neurodegenerative diseases, caregivers are confronted with evolving roles and responsibilities, and need to plan for the trajectory of PD. ${ }^{31}$ Couple-oriented interventions improve spousal coping strategies, minimize stress and anxiety, and increase self-efficacy to manage disease progression. ${ }^{32}$

\section{Disease Progression}

Parkinson's disease-specific sequelae, conditions that result specifically from PD, impact motor and non-motor function. Disease sequelae can present 20 years prior to diagnosis (Figure 1). ${ }^{33}$ Motor and non-motor sequelae can vary depending on the stage of PD. The Hoehn \& Yahr (H\&Y) scale is a five stage scale that examines PD severity based primarily on ratings of motor sequelae. ${ }^{34}$ In stage 1 the patient exhibits unilateral symptoms, while in stage 
2 the patient presents with bilateral symptoms. Those with stage 3 PD experience balance impairment but are still physically independent, while those in stage 4 exhibit severe disability but able to stand or walk unassisted. Stage 5 is the most advanced stage and the person needs a wheelchair or is bedridden unless assisted.

Figure 1: “Clinical Symptoms and Time Course of Parkinson's disease Progression"33

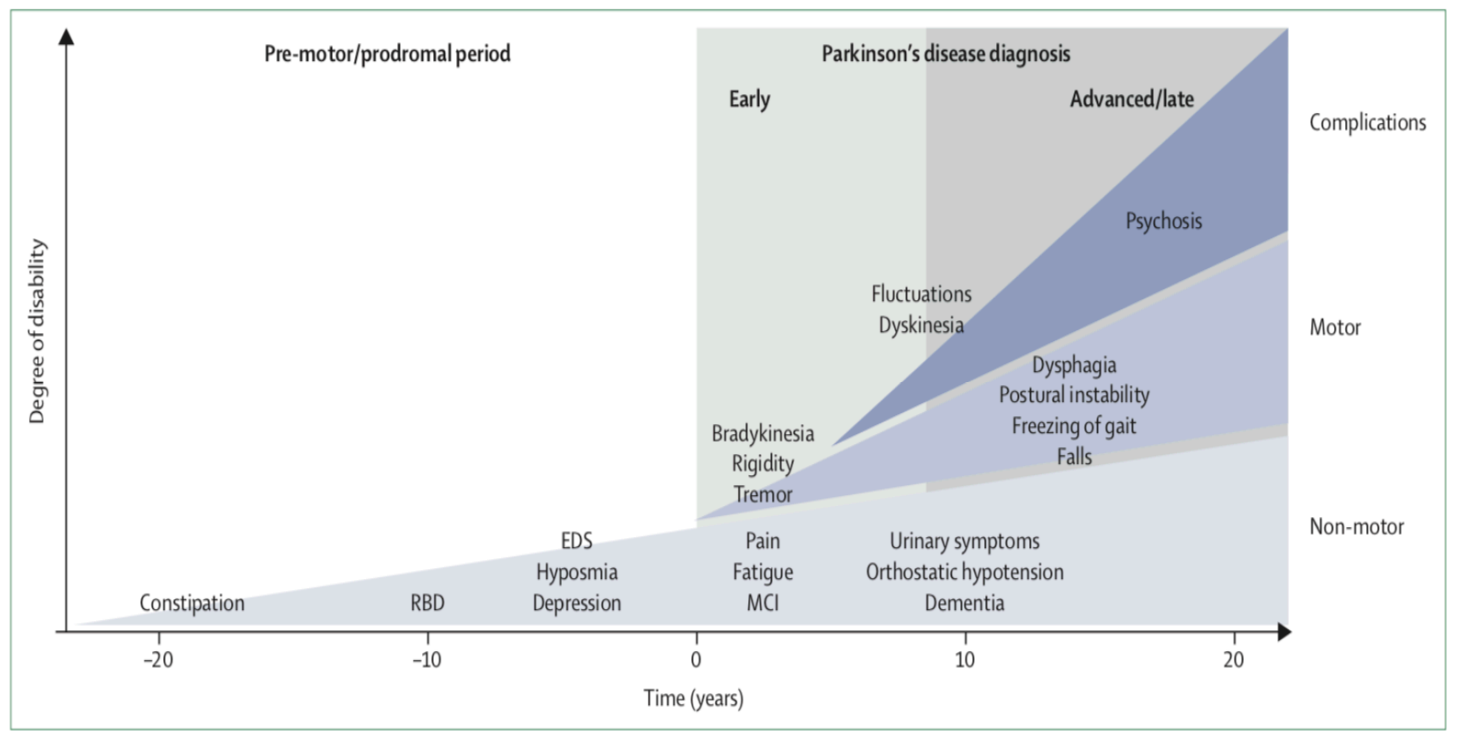

The Unified Parkinson Disease Rating Scale (UPDRS) is a tool to monitor disease trajectory and inform treatment plans for PwPD. The rating scale assess motor and non-motor sequelae and consists of five sections: 1) Mentation, Behavior, and Mood, 2) Activities of Daily Living (ADL), 3) Motor Sections, 4) Modified Hoehn and Yahr Scale (H\&Y), and 5) Schwab and England ADL scale. Higher UPDRS scores indicate more severe disability present, with the highest possible score being 199. ${ }^{35}$ 
Older age at diagnosis and disease duration are independently associated with a higher prevalence of motor and non-motor sequelae that can result in disability. ${ }^{7}$ Due to its heterogenous and degenerative nature, research proposes to view PD as a complex syndrome, rather than a disease ${ }^{36}$ As a result, a comprehensive clinical assessment including biomarkers to assess motor and non-motor symptoms of PD is warranted to better treat and attenuate PD progression. ${ }^{37}$ Tracking how disease sequelae impacts nutritional status overtime is also essential to provide adequate nutrition interventions. ${ }^{38}$

\section{a. Motor Sequelae}

Motor sequelae can be caused as a result of PD or emerge as a side effect of PD medication. ${ }^{35}$ Levodopa-responsive motor sequelae include: dyskinesia (involuntary movement) and motor fluctuations including unpredictable and sudden "off" stage, where levodopa medication suddenly stops working throughout the day. ${ }^{35}$ Non-levodopa-responsive motor symptoms include: tremors, hypomimia (poker face/lacking expression), rigidity, bradykinesia (slowness of movement) and hypokinesia (decreased movement), gait disturbances, freezing gait, balance issues, frequent falls, as well as dysphagia (difficulty swallowing) and speech difficulty. Decline in gait and balance as the disease progresses results in fall frequency. ${ }^{39,40}$ Since so many symptoms do not respond to levodopa treatment, the most effective form of medication for managing PD, an interdisciplinary care team is needed to treat PwPD holistically to manage disease sequelae. ${ }^{41}$ 


\section{i. Physical Functioning}

Motor sequelae progress overtime and can compromise physical functioning..$^{42,43}$ Resting tremor is usually the first and most visible symptom of PD and it impacts one's legs, jaw, tongue and/or hands. Up to $90 \%$ of PwPD experience resting tremor, joint stiffness and/or muscle rigidity ${ }^{44}$. Resting tremor rarely cause disability among individuals since relief or diminution occurs during voluntary movement. ${ }^{44}$ Bradykinesia is one of the most disabling characteristics of PD, impacting up to $90 \%$ of PwPD. ${ }^{45}$ Bradykinesia is disabling as it cause freezing (i.e., sudden, short and transient inhibitions of movements) during other movements, such as walking, driving, talking, moving hands or writing. ${ }^{45}$ Freezing gait is one of the main risk factors for falls among PwPD. ${ }^{46}$ Nearly $68 \%$ of PwPD fall at least once per year ${ }^{47}$, compared

to one third of community-dwelling adults over the age of $65 .{ }^{48,49}$ Bradykinesia can progress into akinesia or the inability to initiate or continue movement. ${ }^{50}$ Akinesia can present as freezing gait (i.e., trouble initiating gait and or turning while walking), speech problems, and incapacity to perform smooth and rapid alternating finger movements. ${ }^{50}$

Change in balance and gait lead to disability among PwPD, compromising ability to perform activities of daily living (ADLs), independence and QOL. Balance and gait issues typically become more compromised as the disease progresses but can also impact physical functioning in earlier stages of disease. Parkinson's disease gait is characterized by slower walking speed, smaller steps, larger stride length variability, and less arm swing 
compared to healthy controls. ${ }^{51}$ Slowing of gait and worsening of motor sequelae over a time and this is a concern among PwPD as it can reduce functional independence, which in turn can decrease quality of life. ${ }^{52}$

Research has also analyzed how physical functioning and ambulatory ability in PwPD changed overtime ${ }^{42,43}$. Cavanaugh et al ${ }^{42}$ conducted a 2-year, prospective longitudinal study, assessing the ambulatory decline and evolving components of disability among 17 PwPD and found the dose and intensity of ambulatory activity significantly decreased. At the same time, the daily dose of levodopa increased overtime. ${ }^{42}$ Findings from this study indicate natural ambulatory activity may be a strong indicator of physical decline, especially in early stages of disease ${ }^{51}$. Natural ambulatory activity is a subset of physical activity behaviors that entail stepping (walking, climbing stairs, mowing the lawn, jogging) and are used as a measurement strategy to assess physical activity, posture and movement. ${ }^{53}$ Findings from this support the need for ambulatory activity monitoring to be included in the PD plan. Research is needed to examine how ambulatory activity changes beyond two years.

Another prospective, longitudinal study observed a significant decline in motor function and self-reported physical activity levels from year two to four in those living with early stage PD. ${ }^{43}$ Higher levels of physical activity among PwPD were significantly associated with ability to perform ADLs, slower progression of motor symptoms and cognitive decline.${ }^{43}$ Physical disability and inability to perform ADLs can also decrease access to healthcare services that would better manage the disease. ${ }^{54}$ 
Finally, States et al..$^{55}$ examined change in physical functioning after one, three and five years of attending an exercise program for PwPD. Fiftynine percent of participants completed one year of the exercise program, while $39 \%$ completed three years and $29 \%$ completed five years. Those participants that were categorized as consistent exercisers (PwPD who completed at least half of the exercise classes for at least one year) showed modest, but significant improvements in hand-grip strength, balance scores, and a sixminute walk test. ${ }^{55}$ However, at years 3 and 5 , no changes in these variables occurred, indicating consistent exercise may help PwPD maintain their functional status despite living with a neurodegenerative disease. Findings from this study support the need for an interprofessional team to successfully implement a supportive community-based exercise program to support participation of PwPD overtime.

Decline in mobility or ambulatory ability as PD progresses can not only increase risk for falls, but may also inhibit one's ability to carry out general (bathing, dressing, eating, toileting, getting in and out of bed, mobility both inside and outside of the home ${ }^{56}$ ) and instrumental ADLs (laundry, preparing meals, shopping, banking, managing money $\left.{ }^{56}\right) \cdot{ }^{57}$ As a result, PwPD may need assistance with buying, preparing, and consuming meals and snacks. Additionally, research has found a relationship between physical activity level and/or physical functioning with cognition and well-being measures such as QOL and depression among PwPD. ${ }^{58,59}$ Such findings support the need for an interdisciplinary treatment approach for managing PD to promote health- 
related QOL. However, more research is needed to understand how this relationship changes over the course of PD. Limited information also exists around the relationship between physical, cognitive, and swallowing functions, and their multi-faceted relationship with nutritional status in PwPD and how this interaction changes overtime. ${ }^{38}$

\section{ii. Swallow Function}

Similar to the decline in mobility, a decline in swallow function can hinder the ability to swallow medication ${ }^{60}$, decrease one's ability to consume nutrient rich foods and compromise nutritional status. ${ }^{61}$ Dysphagia is a condition where one experiences difficulty swallowing food and can occur anywhere between the oral cavity to the stomach;62 including difficulty initiating swallow and getting food stuck in the top or middle of the esophageus. ${ }^{62}$ Oropharyngeal dysphagia, difficulty or inability to chew and mix food with saliva and move it to the back of the mouth with the tongue to the esophagus, is the most common form of dysphagia among PwPD. ${ }^{63}$ Dysphagia among PwPD can be caused by impaired cognitive processing, which can result in difficulty initiating swallowing as a resulting of hypometabolism in the supplementary motor area and dysfunction of the anterior cingulate cortex, causing impaired cognitive processing.$^{64}$ It is estimated that up to $90 \%$ of PwPD will experience dysphagia throughout the course of their disease ${ }^{65}$, while prevalence of dysphagia only impacts $2-16 \%$ of the general population. ${ }^{66}$ Symptoms of dysphagia include regurgitation, chest pain, aspiration, persistent coughs, sore throat, loss of appetite, hoarseness, hiccups, painful 
swallowing, heartburn. ${ }^{62,67}$ Regular swallow screenings are warranted among PwPD, rather than relying on self-reported concerns about swallowing among PwPD. ${ }^{68}$

In a cross-sectional study, Miller and colleagues ${ }^{68}$ examined the $^{-1}$ frequency of impaired swallowing via a $150 \mathrm{~mL}$ timed swallow test, how impaired swallowing relates to disease progression and frequency of selfreported swallowing issues among PwPD. ${ }^{68}$ Results from this study found that $23 \%$ of participants could not completely drink the full $150 \mathrm{~mL}$ glass of water provided during the swallow evaluation and there was a moderate association between swallow function performance and UPDRS II (ADLs) and III (motor function) scores. ${ }^{68}$ Sixty-six percent of participants believed they did not have a swallowing problem but performed below average on the timed-swallow test. $^{68}$

To help manage dysphagia and reduce risk of choking, swallow maneuvers and exercises, postural adjustments, and modified textures (puree, mechanical, soft foods) and fluid consistencies (thin liquid, nectar and spoonthick) can be prescribed. ${ }^{69}$ For PwPD prescribed a dysphagia diet, nutrition guidance is needed to help meet adequate nutrition and hydration needs. ${ }^{70}$ Caregivers may also have a significant role in helping PwPD adhere to a dysphagia diet, especially as the disease progresses and cognitive decline emerges. ${ }^{71}$ The risk of developing dysphagia coupled with nutrition risk present among PwPD warrants the need for ongoing nutrition and swallow screening and collaboration between speech therapists and registered 
dietitians. $^{41}$

Dysphagia can negatively impact dietary intake and increase nutrition risk. One study found that the majority of patients with dysphagia patients do not find mealtime enjoyable and $41 \%$ reported having anxiety or panic during meals. ${ }^{67}$ Since dysphagia can disrupt mealtime, diet quality and nutrition risk is a concern among PwpD. Specifically, the amount consumed, and quality of the food consumed is compromised, which can impact body composition. Consequently, patients with dysphagia present with smaller calf and arm circumference, indicative of muscle wasting and under-nutrition. ${ }^{62}$ Difficulty swallowing among PwPD has been well-studied ${ }^{67}$, but the exact interaction between nutritional status and swallow functioning and how this changes overtime among PwPD has not been examined.

Matushima et al. ${ }^{72}$ conducted a cross-sectional study to assess the association between factors related to swallowing difficulty in 237 PwPD in Japan. This study also wanted to better understand behavior patterns behind the food types selected to cope with malnutrition and describe optimal characteristics of caregivers helping to manage swallowing difficulties their loved-one with PD is experiencing. Findings from this study indicate severity of swallowing difficulties was associated with increased age and more advanced disease. Only 11 participants reported using care foods for managing dysphagia, which included home delivery meals adhering to dysphagia diets or a la carte pre-prepared foods designed for those with dysphagia. The 11 participants consuming care foods were significantly older 
and none of these participants presented with a lower BMI. Dietary modifications to manage dysphagia, were more likely to occur when PwPD lived with an informal caregivers, particularly children. ${ }^{72}$ Findings indicate care foods may be effective in managing dysphagia and nutritional status but research assessing a larger sample of PwPD overtime is warranted. ${ }^{72}$ The issue of care foods and its impact on health-related quality of life (HRQOL) among PwPD is also warranted, as PwPD experience non-motor sequelae, such as depression and apathy.

\section{b. Non-Motor Sequelae}

Non-motor sequelae are being recognized as neglected aspects of PD, and effect up to $88 \%$ of PwPD. ${ }^{73}$ Non-motor sequelae include: change in mood (anxiety, depression, apathy), fatigue, pain, cognitive decline (dementia, memory, concentration attention), psychosis (hallucinations or delusions), excessive sweating, bladder urgency, dizziness, and/or orthostatic hypotension. ${ }^{7,74}$ Many non-motor sequelae among PwPD can impact dietary intake and nutritional status, and include: dysphagia, change in taste and

smell, gastrointestinal issues (gastroparesis, constipation, acid reflux). ${ }^{61,75}$ Dysphagia, while characterized as a motor symptom when assessing PD severity via the UPDRS is considered a mixed motor and non-motor symptom. ${ }^{74}$

A cross-sectional study examining presence of non-motor symptoms among 89 PwPD self-reported a mean of 11 non-motor symptoms.$^{76}$ Nonmotor sequelae tend to vary with fluctuations in motor sequelae associated 
with on/off states ${ }^{74}$, and are key causes of loss of independence and caregiver strain. ${ }^{77}$ In a prospective study, Duncan and colleagues ${ }^{78}$ evaluated impact of non-motor symptoms on HRQOL among newly diagnosed PwPD and found depression, anxiety, poor concentration, memory issues, insomnia and incomplete bowel emptying had the greatest impact on HRQOL. ${ }^{78}$

Subsequently, screening and managing these non-motor symptoms should be prioritized early at diagnosis. ${ }^{78}$

\section{i. Parkinson's Disease, Mood, and Mental Health}

Up to $50 \%$ of PwPD have anxiety, depression, and/or sleep

disturbances. ${ }^{79,80}$ Apathy, depressed mood, and anhedonia (inability to feel pressure) are characteristics for clinical diagnosis of depression and are related the low levels of norepinephrine (hormone), dopamine and serotonin (neurotransmitters) among PwPD and associated with more severe motor sequelae. ${ }^{81}$

Storch et al. ${ }^{74}$ examined the frequency and severity of nonmotor fluctuations among 100 PwPD, as well as the association of nonmotor and motor sequelae and found presence of fatigue, anxiety, depression, and pain is associated with poorer HRQOL independent if non-motor symptoms were present in the on or off state. ${ }^{74}$ Non-motor symptoms were more severe in the off state than the on state. ${ }^{74}$ Fatigue was the most frequently reported nonmotor symptom, impacting $88 \%$ of PwPD. ${ }^{74}$ The second most common nonmotor symptom reported was issues with concentrations/attention, reported by $67 \%$ of PwPD, while dysphagia was least commonly reported $(29 \%){ }^{74}$ 
However, lack of energy may impact the ability to prepare and consume food, while decline in concentration can impact the way nutrition education is presented.

Medication to treat psychiatric conditions can also impact well-being and physical functioning among PwPD. Benzodiazepines, prescribed to treat anxiety, can impact cognition, alertness, and gait, and increase fall and fracture risk. ${ }^{82}$ However, selective serotonin reuptake inhibitors and norepinephrine reuptake inhibitors used to treat depressive symptoms among PwPD can improve depressive symptoms and freezing of gait but have little impact on feelings of apathy. ${ }^{83}$ Unfortunately, gastrointestinal events may be more common in PwPD managing depressive symptoms with SSRIs (nausea, diarrhea, abdominal stomach, vomiting) which can further impact dietary intake and quality. ${ }^{83}$

In addition to non-motor sequelae, PwPD and their informal caregivers also experience stigma as a result of living with a progressive disease, which can further impact well-being. Maffoni et al. ${ }^{84}$ conducted a literature review of qualitative studies examining stigma among PwPD and caregivers and found stigmas included: disgrace, shame, embarrassment, feeling awkward, horrible, terrible or dishonorable. Findings revealed that stigma presents as a complex, multi-faceted construct that is linked not only with the physical decline PwPD experience but also the undesirable self-image and loss of self-efficacy and independence that emerges from the progressive disease ${ }^{84}$; many PwPD felt shame because of physical dependence on caregivers to do even simple 
tasks. ${ }^{84}$ Stigma was also linked to relational and communication problems, where many PwPD attribute voice and articulation sequelae a contributor to stigma. It is important to recognize PwPD and their caregivers journey of living with PD to recognize their inner psychological needs to optimize care provided. ${ }^{84}$

\section{ii. Cognition, Mild Cognitive Impairment and Dementia in PD}

Cognitive dysfunction can occur early in diagnosis ${ }^{85,86}$, and eventually up to $40 \%$ of PwPD present with dementia. ${ }^{79}$ Cognitive symptoms are strongly associated with increased economic burden and nursing home placement, as well as morbidity, disability, and compromised QOL for PwPD and their informal caregivers, increasing caregiver burden. ${ }^{87-89}$ Common cognitive issues that emerge among PwPD include impairments in executive function, working memory and attention. ${ }^{90}$ Kudlicka et al ${ }^{86}$ examined executive function impairment in those living with mild to moderate PD and found PwPD to more frequently have issues with attention control assessments than those assessing abstract thinking performance. Research indicates PwPD with mild cognitive impairment $(\mathrm{MCl})$ are more likely to have issues with performing cognitive set-shifting tasks involved in decision making. ${ }^{91}$ Among PwPD with mild cognitive impairment there is an increased presence of disability and physical impairment, which can impact QOL. ${ }^{25}$ Cognitive deficits among PwPD are predictive of the development of dementia and as a result longitudinal assessment and management of cognition in this population is warrant. ${ }^{90,92}$

Arie and colleagues ${ }^{92}$ conducted a five-year, prospective study of 57 
participants saw a significant decline in cognitive scores in both those with TD and PIGD. However, findings from this study suggest, PIGD participants exhibited a larger magnitude of cognitive decline, particularly in the area of executive function and motor-cognitive skills. Despite these differences, this study found no difference in HRQOL or disease burden between PIGD and TD. ${ }^{92}$ However, future research needs to examine the longitudinal change of nutritional status presents as changes in cognition occur.

In a mixed-methods study, Raein et al. ${ }^{88}$ interviewed dyads comprised of PwPD and their informal caregivers to determine subjective cognitive complaints. Findings from 22 dyads participating in focusing group identified subjective cognitive complaints across the following domains: memory, language and communication, attention and processing speed, executive functioning and episodic confusion/fluctuations in thinking ability. ${ }^{88}$ Dyads described subjective complaints that are not often objectively assessed when measuring cognitive status, such as fluctuations in cognitive abilities, lapses in prospective memory, and issues with recalling names, an issue distinct from an issues with general word-finding. ${ }^{88}$ Those PwPD without dementia reported more subjective cognitive complaints compared to observed cognitive complaints reported by their informal caregiver. ${ }^{88}$ The top treatment priority among PwPD was related to language (recall, word finding, difficulty following instructions), while for informal caregivers the top treatment priority for PwPD were related to executive function (improving decision making abilities and a reduced desire to start tasks). ${ }^{88}$ Findings suggest practitioners should 
consider the unique aspects of cognitive decline in PwPD by examining both subjective and objective cognitive functions. Understanding cognitive deficits and self-reported cognitive concerns can help better design, develop and deliver effective nutrition education.

\section{iii. Sensory and Perception Changes}

People with PD experience sensory changes and reduced insight, which can impact decision making, cognition, physical functioning, swallowing ability and food intake. Such changes include increased saliva production, drooling, change in the ability to taste and smell, altered time and vision perceptions, deficits in perception of loudness, failure to identity emotion and prosody, and inability to change or shift sets quickly. ${ }^{93-96}$ More than 50\% of PwPD report issues with drooling ${ }^{97}$ and approximately a quarter of PwPD experience issues with frequent drooling ${ }^{97}$; people with PD are five times more likely to experience issues with drooling than healthy controls. Drooling frequency is associated with disease severity ${ }^{97}$ Speech language pathologists are critical for the care team in helping PwPD manage drooling. Changes in tactical function, thermal, nociceptive (perception or pain), and proprioceptive (perception of self-movement and body position) sensations also occur among PwPD. ${ }^{96}$

These sensory changes among PwD are either pure disorders of conscious perception or disorders of sensorimotor integration ("the use of sensory information to guide movement"). ${ }^{96}$ Disorders of conscious perception can cause elevations in the sensory threshold, while disorders of 
sensorimotor integration occurs when there is an alteration in the interaction between sensory input and motor output. ${ }^{96}$ Despite normal cognitive functioning, PwPD have a decreased sensitivity to visual sensory and cognitive stimuli, which implies alterations to the visual cortical and subcortical areas, along with possible impacts on the retina. ${ }^{98} \mathrm{It}$ is also speculated that dopaminergic treatments worsen sensory impairments (e.g. postural instability), by disrupting the primary somatosensory cortex, found in the postcentral gyrus of the brain. ${ }^{99}$ As a result, how PwPD perceive situations, their own health status and dietary intake, as well as process nutrition and health education may be impacted. Multiple modalities and repetition to educate PwPD may be warranted. ${ }^{100}$

Cognitive-linguistic changes can impact QOL and day-to-day functioning of PwPD. While nearly $75 \%$ of PwPD experience a speech disorder at some stage in the disease, PwPD have an impaired perception of their own speech loudness as well as impaired perception of verbal emotions when interacting with others. ${ }^{94}$ It is proposed that altered perception of own speech loudness is due to the inconsistency between perceived level of effort and produced vocal loudness. ${ }^{101}$ Meanwhile, inability to detect verbal emotion may be due to compromised working memory, executive function, and acoustic processing. ${ }^{94,102}$ As a result, on-going monitoring cognitive status among PwPD is vital. This information is also important for health professionals providing ancillary services and education to PwPD to design and implement effective services. 
In addition to lack of insight about perceived loudness and interpretation of emotional parody, PwPD also can have challenges with shifting tasks and perception of time. ${ }^{93,95}$ When examining the change in muscle responses when provided a cue to complete two different tasks, PwPD needed to perform the original task several additional times before switching to the new task. ${ }^{95}$ Those PwPD taking levodopa did not see improvements in their ability to change tasks quickly. ${ }^{95}$ Findings of this study should be taken into account when designing nutrition education and assessment programs for PwPD. Temporal information processing is another sensorimotor integration process altered among PwPD and may explain some PD symptoms, such as poor time perception. ${ }^{84}$ Research has found PwPD tend to underestimate time, and it is proposed dopamine deficit may cause a delay in the body's internal clock.

Distortion of time is a concern among PwPD as time perception is fundamental for the relationship between humans and their environment. As a result, how PwPD perceive their surroundings and experiences may be altered. How alterations of perceptions among PwPD impact nutrition assessment, dietary recalls and nutrition education has not been explored. Changes in perception also warrant the inclusion of caregivers to provide insight on the diets of their loved one living with PD.

III. PD Management

a. Treatment Options for Managing PD

i. Pharmacologic Anti-Parkinson Treatment 
Dopamine Agonists. Dopamine agonists, while less effective and potent than levodopa, are usually used as the initial medication for PD. ${ }^{9}$ Compared to levodopa, they are associated with a lower risk of motor fluctuations and dyskinesia for the first five years of treatment. ${ }^{9,103}$ Dopamine agonists bind to dopamine receptors to mimic the neurotransmitter dopamine. ${ }^{104}$ However, overtime levodopa is usually used in conjunction with dopamine agonists to control advancing symptoms. ${ }^{9}$ Those on dopamine agonists are more likely to experience non-motor side effects compared to those on levodopa (Figure 2). ${ }^{105}$ Nutritional side effects of dopamine agonists include weight gain and compulsive behaviors, such as excessive spending and eating, which can impact health-related QOL among PwPD. ${ }^{9,61}$

Figure 2: Incidence of adverse effects in Parkinson's disease for trials of Dopamine Agonists (with and without levodopa) versus Levodopa Alone ${ }^{105}$

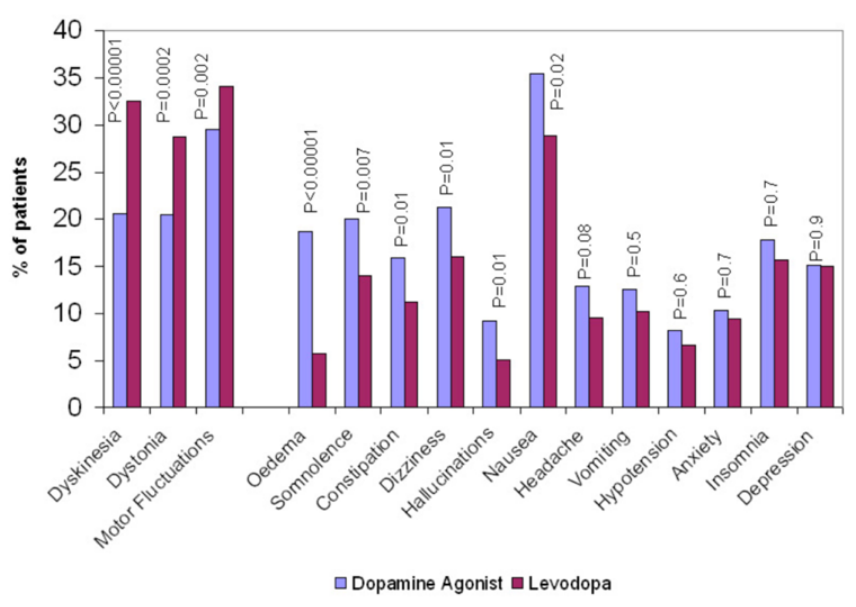

Levodopa Containing Medications. Levodopa is the most effective medication for treating PD motor sequelae, because it improves function, $\mathrm{QOL}$ and reduces morbidity and mortality among PwPD. ${ }^{18}$ The medication is 
converted to dopamine in the brain and is administered to increase striatal dopamine levels. ${ }^{18}$ It's tolerability and efficacy was improved by combining levodopa with dopa-decarboxylase inhibitor (carbidopa-levodopa). ${ }^{18}$ While levodopa is the gold standard for managing PD motor sequelae, overtime PwPD can build a tolerance to levodopa and the medication can become ineffective. The response time where levodopa is effective and motor sequelae is minimized is known as the "on" time. The "off" time is the period before the next levodopa dose is consumed and motor sequelae are present. Figure 3 highlights the change in levodopa response over the progression of PD. ${ }^{18,106}$ Those with early stage PD seem to have a prolonged response to levodopa containing medication with longer "on" times and shorter "off" periods. As the disease progresses, the short half-life of levodopa and increase tolerance, medication duration of action and motor benefit wears off quicker before the next dose is scheduled. In advanced PD, adverse effects of levodopa, such as dyskinesias during the "on" time emerge and levodopa becomes ineffective. As a result, PwPD have to take levodopa more frequently and often at higher doses. ${ }^{18}$ To optimize the effectiveness of levodopa, levodopa containing medications are not usually prescribed in the early stages of PD and introduced later as motor sequelae become more pronounced. Levodopa also competes with absorption with neutral amino acids found in animal protein, and if consumed with high protein meals the medication is less effective. People with PD taking levodopa-containing medication are encouraged to wait 30-minutes to an hour between consuming a high protein meal and taking their 
levodopa containing medication. ${ }^{61}$ As a result, working with a Registered Dietitian $(\mathrm{RD})$ to manage medication and nutrient timing is essential to prolong the effectiveness of levodopa-containing medication. ${ }^{41}$

Figure 3: Change in Levodopa Response Over the Course of PD 18,106
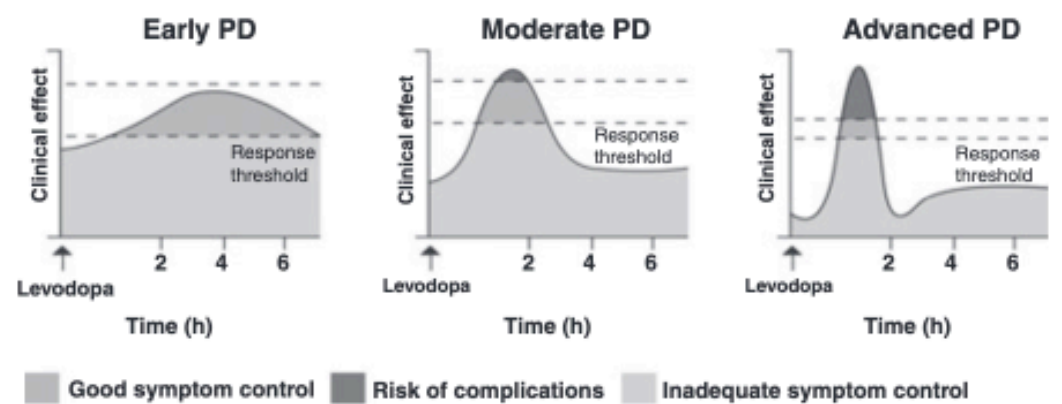

Smooth, extended duration of target clinical response

Low incidence of dyskinesias

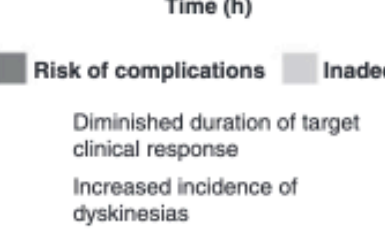
Short duration of target clinical response
'ON' time is associated with dyskinesias

Other Pharmacologic Treatments for PD. Anticholinergic agents and MAO-B Inhibitors can also be prescribed to treat PD but are less common due to their adverse effects or ineffectiveness. Side effects of anticholinergic agents include confusion, impaired memory, constipation, blurred vision, urinary retention, dry mouth and glaucoma. ${ }^{9} \mathrm{MAO}-\mathrm{B}$ Inhibitors, while produce less side effects, are ineffective when used alone to manage PD. Side effects of MAO-B Inhibitors include insomnia, nausea, anorexia, hallucination, and potential interaction with medications such as selective serotonin reuptake inhibitors and meperidine. Many PwPD receive a combination of medications to manage PD which may have side-effects that impact, dietary intake, medication-interactions and health-related quality of life. These side-effects can impact adherence to medication and working closely with a neurologist, pharmacist and RD can help optimize medication effectiveness. 
Pharmacologic Adherence. People with PD taking anti-parkinson medication often need to take a steady level of medications throughout the day. As PD advances medication management becomes more complex and PwPD may have to take anti-parkinson medication every 2-3 hours. However, with advanced disease, motor and cognitive deterioration may make adhering to such complex medication treatment too difficult for PwPD on their own, resulting in greater amount of time, effort and family support needed to manage medications regiments. ${ }^{107,108}$ In the US it is estimated that $27.3-67 \%$ of PWPD have poor adherence to anti-parkinson medication management. ${ }^{108-}$ 111

In an exploratory, qualitative study, Shin et al ${ }^{108}$ interviewed 16 PwPD and five informal caregivers to better understand challenges to anti-parkinson medication adherence and strategies used by PwPD and caregivers to overcome these challenges. Challenges among participants included medication responses, cost of medication, or forgetting to take medications. ${ }^{108}$ Those who described medication response as a reason for missing doses was because they did not notice any difference or effects after taking their antiparkinson medication. ${ }^{108}$ Several participants also noted that the cost of medication was a burden, despite coverage from their insurance company. Participants also had a concern about protein and levodopa interaction, describing uncertainty about the time they were supposed to wait to consume a high protein meal after taking medication and difficulty scheduling daily routines around meal times. ${ }^{108}$ Finally, a few participants reported not taking 
their medication at the prescribed time because they forgot. ${ }^{108}$ Strategies used by PwPD and caregivers to facilitate medication adherence included seeking knowledge about the medication, seeking advice from family and friends, using pillboxes to keep track of medications or using alarms and smartphones to set reminders to take their medication. ${ }^{108}$ Findings from this study can help inform medication adherence interventions among PwPD to minimize food-drug interactions and optimize medication effectiveness. In addition to using smart phones for medication adherence, digital health technologies, such as cloud technologies are being used internationally to promote medication safety and adherence in aged societies. ${ }^{112}$ Such findings advocate or exploration of how cloud technologies and digital health may support medication safety and adherence among PwPD. ${ }^{112}$

\section{ii. Deep Brain Stimulation}

Deep brain stimulation (DBS) is an established and effective treatment strategy for those living with advanced PD. ${ }^{113}$ This surgical procedure involve implanting a device that delivers small electric shocks to areas of the brain. In PwPD the target areas of the brain include the subthalamamic nucleus (STN) or the globus pallidus pars interna (GPi). ${ }^{113}$ Deep brain stimulation can effectively help manage motor fluctuations and dyskinesias. Findings found the motor benefits from DBS were sustained over 36 months and longer longitudinal evaluation is warranted. ${ }^{113}$ When targeting STN specifically, there is a significant reduction oral medication required among PwPD. ${ }^{113}$ However, DBS targeting the STN does seem to improve depressive symptoms to the 
significant extent as stimulation to the Gpi. ${ }^{113}$ Research suggests veterans with PD who received DBS compared to those who received usual care have longer survival rates. ${ }^{114}$ Stroupe and colleagues ${ }^{115}$ compared the healthcare utilization and costs of veterans with PD who received DBS compared to those who did not over five-years and found average healthcare costs for veterans who received DBS were $\$ 77,131$ higher ( $\$ 162,489$ vs $\$ 85,358)$. However, when excluding costs for DBS procedures and complications, there was no significant difference in average total healthcare costs between veterans that received DBS and those who did not. ${ }^{115}$ When assessing the economic value of DBS, a cost benefit analyses should be taken into consideration, including the survival benefits of DBS. ${ }^{114,115}$ In addition to medication and surgical treatment for managing PD, ancillary services can help curtail disease progression.

\section{b. Ancillary Services for Managing PD}

Rehabilitative and complimentary medicine programs are being promoted to compliment pharmacological and DBS treatment. ${ }^{41}$ This includes physical therapy and speech therapy, occupational and exercise regiments. ${ }^{41}$ Lee Silverman Voice Therapy Big (LSVT BIG Therapy) consists of five tasks focusing on large trunk and extremity functional movements, ${ }^{116}$ and requires PwPD to attend four sessions per week for four weeks. ${ }^{117}$ The therapy improves physical functioning among PwPD and helps manage PD related feelings of fatigue and depression. ${ }^{118}$ Exercise programs help prevent falls, optimize body composition, and improve physical functioning and ability to 
perform ADL. ${ }^{119}$ Exercise programs that can benefit physical functioning and overall health of PwPD include: treadmill ${ }^{120}$ and resistance training ${ }^{121}$, Tai Chi $^{122}$, dancing ${ }^{123}$, biking ${ }^{124}$ and boxing. ${ }^{125}$

Visits with a speech language pathologist (SLP) are also a vital part of the PD treatment plan, as the SLP can evaluate, diagnose and treat swallowing and cognitive-linguistic issues that develop among PwPD. ${ }^{126}$ The SLP is also vital in prescribing appropriate modified textured and fluid consistencies to help reduce risk of choking and aspiration pneumonia. An SLP can also provide swallowing exercises to strengthen muscles related to swallowing and improve QOL. ${ }^{127}$ The SLP can also treat speech and voice disorders that emerge from PD, which include mono-pitch, reduced loudness, slowed and slurred speech or inaccurate articulation. ${ }^{128}$ Lee Silver Voice Treatment LOUD (LSVT LOUD) is an effective form of speech therapy for managing speech disordered related to PD. ${ }^{128}$ The treatment requires PwPD to attend one-on-one sessions four days a week for four weeks. ${ }^{128}$ The onehour sessions have been found to increase vocal loudness and functional communication among PwPD. Both LSVT Loud and BIG require tone ups and to keep up with the exercises in between the 4-week sessions. ${ }^{129}$ For PwPD to have added benefits of such programs, high frequency and repetition is needed and this can be time consuming for PwPD and their informal caregivers and prevent PwPD from accessing an interdisciplinary treatment plan. 


\section{c. The International Classification of Functioning, Disability and Health}

The International classification of Functioning, Disability and Health (ICF) is framework approved by the World Health Organization to describe and classify functioning and disability among adults and children living with health-related conditions. ${ }^{130}$ The ICF can be used with the International Classification of Disease and Related Health Problems (ICD) to provide a comprehensive picture of a person's health. Health is defined by the World Health Organization as "the complete physical, mental and social functioning of a person and not merely the absence of disease. ${ }^{130 "}$ With this proposed definition, ICF is an essential component. ${ }^{130}$ The framework can be used to in interprofessional practice, to promote person-centered care and to establish a common language across disciplines, including for those working with PwPD. ${ }^{130,131}$

The ICF framework is broken down into two major components: 1. Functioning and Disability and 2. Contextual Factors. Figure 4 has been adopted to capture the major components when working with PD. ${ }^{130}$ Functioning and disability factors include: body functions and structures, activity and participation. ${ }^{130}$ Body functions and structure describe the actual physical and psychological status of the person. While activity and participation describes their ability to function and participate in desired activities. Functional status includes mobility, communication, interpersonal relationships, ability to safely feed oneself, self-care, and knowledge 
acquisition and application. ${ }^{130}$ Contextual factors include environmental

factors and personal factors. Environmental factors are those factors that can

influence one's health that are not within the person's control (family,

government policies, cultural beliefs and work). Personal factors (race, gender, age, education level and coping styles) may influence how a person manages

their disability and activities they participate in. ${ }^{130}$ The domains within each

factor are highlighted in Figure 5.

Figure 4: World Health Organization's 2001 ICF Model Adopted for PwPD ${ }^{130}$

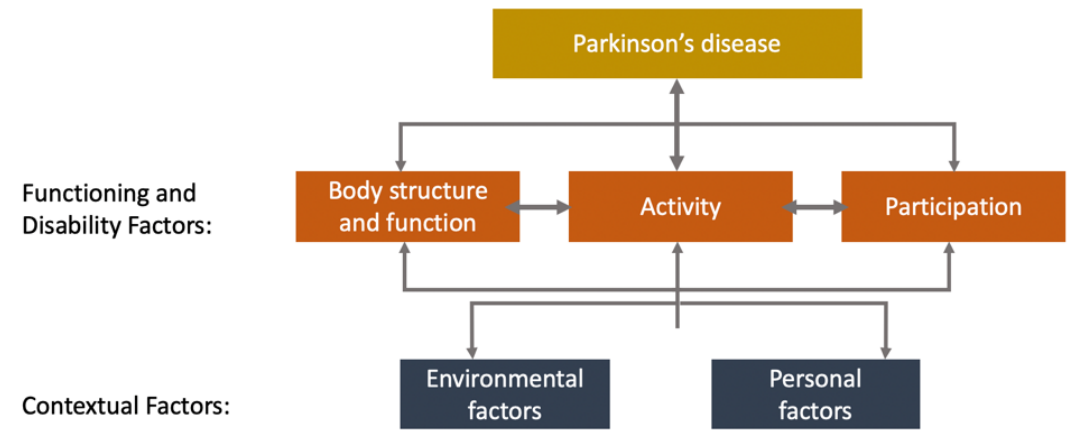

Figure 5: World Health Organization's 2001 ICF Major Components and Domains ${ }^{130}$

\begin{tabular}{|l|l|}
\hline Body Function: & Activities and Participation: \\
Mental functions & Learning and applying knowledge \\
Sensory functions and pain & General tasks and demands \\
Voice and speech functions & Communication \\
Functions of the cardiovascular, haematological, & Mobility \\
immunological and respiratory systems & Self care \\
Functions of the digestive, metabolic, endocrine systems & Domestic life \\
Genitourinary and reproductive functions & Interpersonal interactions and relationships \\
Neuromusculoskeletal and movement-related functions & Major life areas \\
Functions of the skin and related structures & Community, social and civic life \\
\hline $\begin{array}{l}\text { Body Structure: } \\
\text { Structure of the nervous system }\end{array}$ & Environmental Factors: \\
The eye, ear and related structures & Products and technology \\
Structures involved in voice and speech & Natural environment and human-made changes to \\
Structure of the cardiovascular, immunological and & environment \\
respiratory Systems & Support and relationships \\
Structures related to the digestive, metabolic and & Attitudes \\
endocrine systems & Services, systems and policies \\
Structure related to genitourinary and reproductive systems & \\
Structures related to movement & \\
Skin and related structures & \\
\hline
\end{tabular}


Vojciechowski and colleagues ${ }^{131}$ completed a literature review to describe and quantify the development use of IFC and portray the different components of IFC that have been used in PD. Only four studies have examined the relationship between IFC and PD. Questionnaires and assessments administered among PWPD and their relationship between ICF domains are summarized in Figure 6. ${ }^{131}$ Findings from this review concluded more research is needed to better examine the association between the ICF and PD. ${ }^{131}$ Additionally, ICF and its relationship to PD outcomes following a health intervention is also warranted. ${ }^{131}$ Adoption of the ICF for PD and understanding the relationship between ICF and PwPD outcomes, can help better track changes among PwPD and provide a universal language between the healthcare team, and promotes PwPD and their caregivers at the center of the careteam. ${ }^{130}$

Figure 6: Questionnaires and Function Tests Related to the ICF Domains and $P D^{131}$

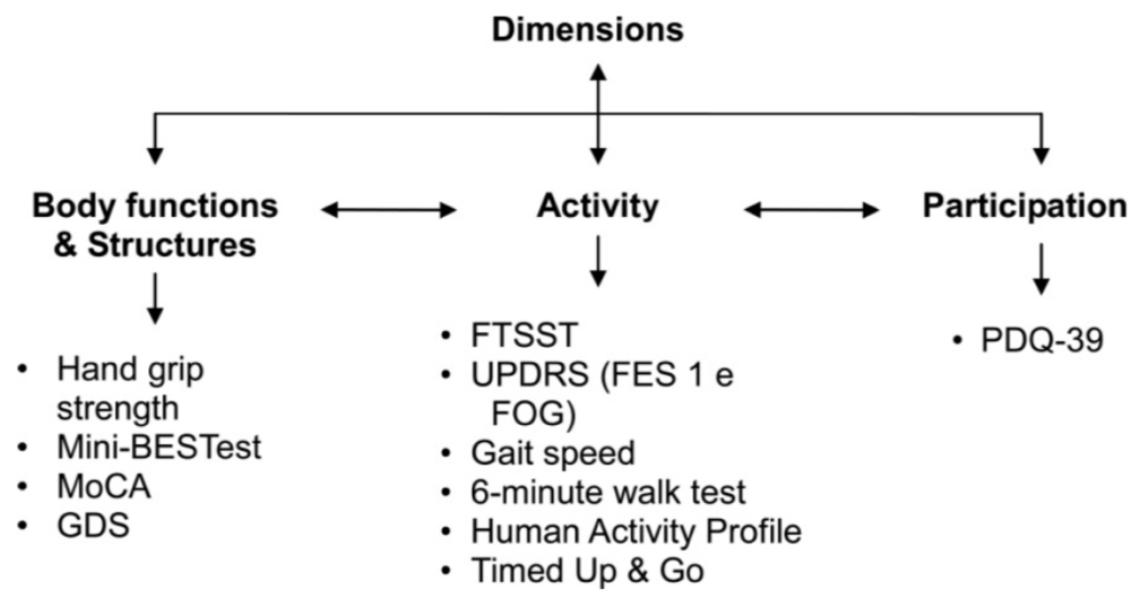

Abbreviations: Mini-BESTest=balance, evaluation systems test; MoCA=Montreal cognitive assessment; GDS=geriatric depression scale; FTSST=five times sit to stand test; FES 1 e FOG=falls efficacy scale, freezing of gait; $P D Q-39=P D$ questionnaire-39 
Components of the ICF model cover aspects of health-related QOL and allows for interaction between domains as a result ICF can serve as a predictor for health-related QOL and health status. ${ }^{58}$ The model is encouraged to be used by physical therapists among PwPD to guide daily functioning and identify specific needs. ${ }^{58}$ How ICF can be used across other help professionals, such as RDs, for the treatment plan of PwPD and impact health-related QOL needs further explanation. In a prospective, two-year study, Cavanaugh et al examined clinical measures associated with ambulatory activity decline among PwPD. The clinical measures assessed fit into the major domains of the ICF model (Figure 7). Findings from this study showed the potential to use ICF model to track long-term ambulatory function among PwPD. Utilization of this model assessing and tracking PD progression over longer periods of time is warranted.

Figure 7: Study measures for PwPD according to ICF model domains

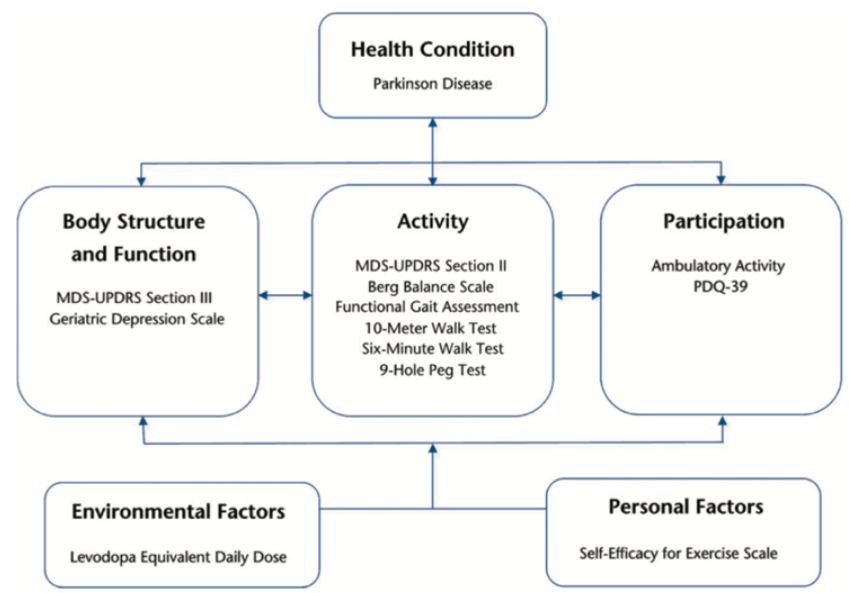

Figure.

Study measures categorized according to International Classification of Functioning,

Disability and Health model domains. MDS-UPDRS=Movement Disorder Society-

Unified Parkinson's Disease Rating Scale, PDQ-39=39-item Parkinson's Disease Questionnaire. 


\section{d. Care Model for Managing PD}

Worsening of disease sequelae overtime, combined with declining nutritional status negatively impact $\mathrm{QOL}^{132}$, which can further compromise ability to perform ADL, making PwPD more reliant on caregiver. ${ }^{133,134}$ Due to this increase reliance, caregivers are essential to healthcare and can partner with healthcare providers to more effectively implement treatment and promote adherence of PD treatment. ${ }^{70,135}$ Including caregivers in interventions can address some of the nutritional concerns PwPD face, allowing caregivers to help maintain the health of PwPD and their own health. ${ }^{41,70}$ Interdisciplinary care is recommended for managing PD, but nutritional care and inclusion of informal caregivers is often overlooked; Figure $\mathbf{8}$ has been modified to include informal caregivers and nutrition services in interdisciplinary PwPD care. ${ }^{136}$

Figure 8: Modified Interdisciplinary Service Model for PwPD ${ }^{136}$

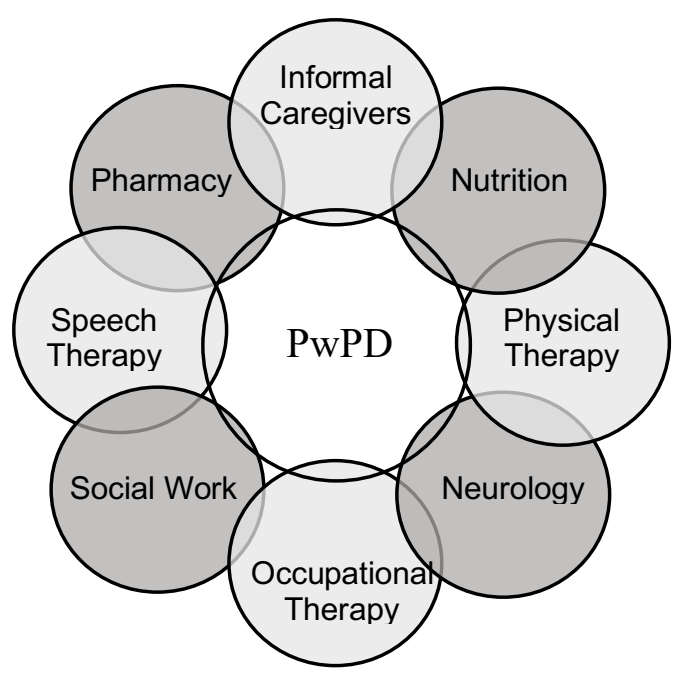


In a mixed-methods study, Kessler and colleagues ${ }^{137}$ surveyed 57 PwPD and 30 caregivers and interviewed 13 PwPD, six caregivers, and six healthcare providers to develop an integrated care program for managing PD. The development of these program advocates for a collaborative approach, integrating PD and caregivers as an active part of managing PD. The collaboration calls for self-management, support and communication with and between healthcare providers. To create this tertiary PD clinic, co-design was utilized, which included viewpoints of key stakeholders, including PwPD and caregivers. Promoting self-management of care provides PD dyads with the skills and confidence needed to manage a chronic health condition on a day to day basis. People with PD and caregivers wanted more support with goalsetting to promote self-management, follow up with healthcare providers and access to services to better manage PD. Both PwPD and caregivers expressed satisfaction when they were able to make decisions collaboratively with their health care providers. Findings from this study should be considered when designing healthcare services for PD dyads to promote access to comprehensive care.

\section{e. Resource Availability}

Since many PwPD have limited access to healthcare due to disease sequelae, age and location ${ }^{138,139}$, an improved model to address healthcare access and need is critical. ${ }^{138,139}$ People with PD experience cognitive and physical decline that can impact mobility and driving ability, which in turn can reduce access to medical care ${ }^{54}$ While more research is needed to determine 
if there is a higher occurrence of PD among rural vs urban populations, research has found a link between occupational exposures and increased incidence of PD. These occupations that appear to have an increased occurrence of PD include agriculture, working with pesticides, and heavy metals. ${ }^{140}$ Those PwPD living in rural areas are especially at increased risk for inadequate access to treatment.

According to the current interdisciplinary care model, PwPD should see a neurologist 2-4 times a year ${ }^{141}$, and physical therapists and speech language pathologists up to 4 times a week for certain treatments. ${ }^{54,142}$ This can be burdensome for PwPD and their caregivers, due to limited access to transportation and compromised physical mobility. ${ }^{50}$ Up to $40 \%$ of Medicare beneficiaries with PD do not seek care from a neurologist within the first four years after diagnosis. ${ }^{138}$ Additionally, while physical and speech therapies exist to help manage PD sequelae, there are no recommendations for routine appointments with allied health professionals, including speech-language pathologists, physical and occupational therapists, exercise physiologists and/or registered dietitians (RDs).$^{41}$ Monitoring how PwPD change across the domains of nutrition, cognitive-linguistic and physical functioning can help better understand health needs of PwPD. ${ }^{38}$ Inclusion of allied health professionals can help attenuate burden of disease sequelae, improve QOL and may lower healthcare costs. ${ }^{143}$

Due to limited mobility, visuospatial impairment, decreased access to transportation options, need for visual assessment and on-going care, PwPD 
are excellent candidates for digital health. ${ }^{26,139}$ However, nutritional services via digital health are not utilized in this population and the individualized nutrition care needed by PwPD is also an under-recognized component of care. ${ }^{144}$ Monitoring the nutritional status of PwPD and caregivers can help better understand nutritional needs and provide effective digital health nutrition services. Tracking how PwPD change overtime can also help monitor and track treatment efficacy provided across disciplines and further promote interprofessional practice to manage PD.

I. Theoretical Framework: Technology Acceptance Model Obtaining PwPD preferences and opinions regarding features of technology to manage health can help to inform the creation of a user-friendly, evidenced-based digital health service. ${ }^{145}$ Formative analysis has been used to effectively employ digital health technologies for older adults ${ }^{146,147}$, and in the development of dyadic interventions for PwPD. ${ }^{148}$ Understanding PD dyads preferences for technology to manage and track nutrition health can be incorporated into the technology acceptance model (TAM). ${ }^{149}$

The TAM was created to explain computer usage behavior across a broad range of user populations and end-user technologies. ${ }^{149}$ The model seeks to provide a basis for understanding external factors that influence internal beliefs, attitudes and intentions to use technology (Figure 9). ${ }^{149}$ External factors can directly impact perceived usefulness and perceived ease of use related to various computing technologies. The model hypothesizes that perceived usefulness and perceived ease of use jointly determine acceptance, 
which can influence intention to use and actual behavior use of technology. ${ }^{149}$ The TAM has also been modified to specifically examine technology acceptance among older adults, known as the Senior Technology Acceptance Model (STAM). The external factors STAM examines specifically relate to aging populations that may impact technology use and attitudes toward technology. ${ }^{150}$ These external factors include: self-efficacy to learn technology, anxiety toward technology, facilitating conditions, self-reported health conditions, cognitive ability, social relationships, attitude to life and satisfaction and physical functioning. ${ }^{150}$ External factors that impact PD dyads to use technology are important to understand to ensure usability and utilization of digital health for managing PD. ${ }^{150}$ As a result, including PwPD and caregivers in the formative stages of research can facilitate the creation of user-friendly digital health nutrition services. ${ }^{145}$

Figure 9: Technology Acceptance Model ${ }^{149}$

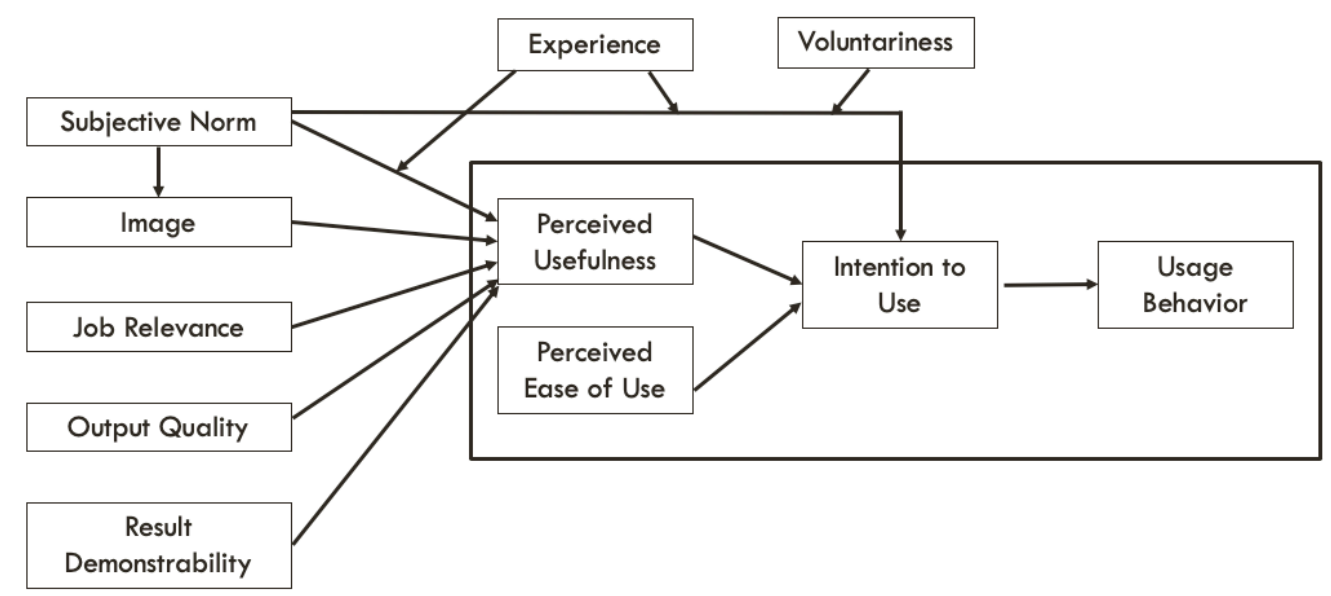




\section{Digital Health}

\section{A. Overview of Digital Health}

Improvement in the current healthcare model for PwPD is needed to effectively meet patient needs. ${ }^{54,138}$ Digital health describes technologies that better manage and track health. ${ }^{151}$ Additionally, as technology adoption increases, eight in ten (113 million internet users) seek health information online. ${ }^{152}$ Specifically, $64 \%$ of adults living with one or more chronic diseases seek health information from online sources. ${ }^{153}$ Most US adults (77\%) report owning a smart phone device. ${ }^{154}$ Recent efforts have been made to develop and use digital health to assess, monitor, and provide therapeutic treatments for PwPD. ${ }^{26,155}$ Such efforts can increase access to care and promote efficiencies in providing care. ${ }^{156}$

More than $40 \%$ of PwPD are over the age of 65 , and most PwPD living in rural areas will not see a neurologist after diagnosis. ${ }^{138}$ Forty-percent of Medicare beneficiaries with PD do not seek care from a neurologist within the first four years after diagnosis. ${ }^{138}$ In addition to visits with neurologists 2-4 times per year ${ }^{141}$, multidisciplinary care models, including visits with physical therapists and speech language pathologists, are encouraged and effective. ${ }^{157,158}$ Some of these treatments require sessions four times per week $^{142}$ which can be a challenge for those with limited access to transportation and compromised physical mobility, thus becoming burdensome for PwPD and caregivers. ${ }^{54}$ Disease stage is associated with driving safety, where stage 3 is significantly associated with a decline in driving ability. ${ }^{159}$ Due 
to immobility, limited means of transportation, and ongoing multidisciplinary care, PwPD are excellent candidates for digital health. ${ }^{139}$

Digital health serves as a gateway to efficacious and convenient health information and treatment. ${ }^{156}$ Digital health increases access to health services, decreases healthcare inefficiencies, offers more personalized services, and reduces burden and medical costs for those living with neurological disorders, including PD. ${ }^{156}$ Technological advancements such as cloud computing, sensors/wearable devices, mobile and video communications $^{160,161}$, and social networks ${ }^{151}$ increase access and quality of care for PwPD. ${ }^{26}$

In addition to usability and increasing access to care, digital health adoption can help minimize healthcare costs. In 2012, the Veterans Health Administration served 150,000+ telehealth beneficiaries, and estimated an annual savings of $\$ 6,500$ per user; a billion dollar savings system-wide. ${ }^{162}$ People with PD that have utilized digital health services have saved on miles traveled and commute time to healthcare providers. ${ }^{156}$ Specifically, PwPD saved 100 miles of travel and three hours of time compared to in person visits. 156 Patients and clients are interested in utilizing digital health, and high patient satisfaction is reported by those PwPD who have utilized digital health. ${ }^{156,163}$

\section{A. PwPD Viewpoints on Digital Health}

Interests and opinions of digital health among PwPD and their caregivers have been assessed. A recruitment webpage for a US-based 
randomized control trial utilizing virtual house calls for PwPD received 11,000 individual views worldwide. ${ }^{164}$ Caregivers are also receptive and willing to pay for technologies that monitor and support care recipients. ${ }^{165}$ Specifically, $20 \%$ of informal caregivers were willing to pay for kitchen (e.g., technologies that assist with meal preparation and washing dishes) and self-care activities (e.g., technology to help the care recipient getting in and out of bed, dressing, eating, bathing, or toileting). ${ }^{165}$ Of those caregivers willing to pay for such technologies, these caregivers reported willingness to pay 50 dollars per month for monitoring technologies and 70 dollars per month for technologies that both monitor and provide assistance to the care recipient. ${ }^{165}$ Findings indicate that a combination of private pay and government subsidy may promote development and adoption of these technologies among informal caregivers. ${ }^{165}$ Experts suggest when designing digital health interventions specifically for PwPD, designed and developers should consider caregiver views, needs and preferences. ${ }^{166}$

A large cohort study measured the proportion of PwPD interested in telehealth (i.e., interactive videoconferencing with a healthcare provider) and identified predictors of patient interest to use telehealth. Among PwPD who currently use telehealth services, $85 \%$ chose to continue utilizing the service. ${ }^{167}$ The five patients who discontinued telehealth were treated with deep brain simulation (DBS), who required trained professional to be present during the visit due too the lack of training/experience of the nurse/technician with telehealth to manage DBS equipment and monitoring DBS programming. 
Approximately $65 \%$ of participants who continued to use telehealth reported they wanted a combination of in person and remote services. ${ }^{167}$ Of those PwPD who had never used telehealth before, $53 \%$ were interested in using the services, believing utilization of the service could result in significant cost savings. ${ }^{167}$ Others interested in telehealth were in the early stages of PD and believed physical examinations by a physician were not necessary at every appointment, so telehealth could serve as check-ins with their physician. ${ }^{167}$

In contrast to these findings summarized above, a cross-sectional survey assessing PwPD views on using different electronic mediums for communicating and exchanging information with healthcare providers, found PwPD may have a less favorable views around technology for communicating with health providers. ${ }^{168}$ Nearly $65 \%$ of PwPD reported they would be willing to use electronic methods and $48 \%$ indicated that using technology to communicate with providers would help PwPD better understand their care. People with PD were asked which forms of technology would help with care. Of the modalities suggested, $\sim 15 \%$ supported electronic forms at check-in for medical visits and $40 \%$ stated they would like a summary of care/home instructions emailed to them. Of the participants surveyed, $\sim 35 \%$ noted they would like the ability to communicate with healthcare providers through email and $33 \%$ indicated they would like video education about services offered to PwPD. ${ }^{168}$ When stratifying participants by age, those over 65 and over were less likely to believe using technology to communicate with a health provider would enhance their understanding of care. Those 75 years of age and older 
had a lower odds of being willing to use electronic methods and believing that technology would result in better understanding of medical needs or their healthcare providers better understanding their medical needs. ${ }^{168}$ Findings from this study indicate optimal communication mediums among PwPD may vary on patient demographics and that training older PwPD to use technology may be warranted. Qualitative interviews are needed to better understand technology choices and preferences of PwPD. Additionally, research that has implemented digital technology among PwPD shows implementation can be advantageous to PwPD and their informal caregivers, including reduced burden and timed saved, which further promotes digital health as a efficacious medium for delivering healthcare services. ${ }^{169}$

\section{B. Efficacy of Digital Heath for Managing Parkinson's}

Digital health, such as telemedicine, has been used successfully in a variety of populations that have evaluated usability (Figure 10). ${ }^{170} \mathrm{~A}$ review of 138 studies was conducted, eight included end-users with neurodegenerative diseases, including PD. ${ }^{170}$ People with PD have used telehealth for visits with specialty physicians and multidisciplinary care providers, such as physical therapy, speech therapy and mental healthcare. ${ }^{142,156}$ Digital health mediums used for managing PD include wearable devices ${ }^{171}$, telehealth ${ }^{142,163}$, and online communities. ${ }^{172}$ Telehealth, or interactive videoconferencing, provide healthcare providers visual cues of patients, which makes the visit more objective $^{139,173}$. Wearable devices collect continuous data to provide a more realistic portrayal of PwPD's daily behaviors and clinical outcomes overtime 
unlike subjective data or cross-sectional assessments. ${ }^{171,174}$ Telehealth can also provide social support and reduce burden and cost for PwPD and caregivers. ${ }^{175,176}$ For example, speech therapy for PwPD via telehealth saved each caregiver 48 actual hours involved in a speech therapy visit, 92 hours of work time (time taken off from work), and over $\$ 1000 .{ }^{169}$ In addition to economic benefits and reduced participation burden, telehealth increases access to health information, provides effective quality of care, and influences individuals to make healthy lifestyle choices. ${ }^{177,178}$ Telehealth is believed to motivate patients to increase access to care, achieve greater control over disease management, and serves as an effective medium for healthcare management in older adults. ${ }^{179-181}$

Figure 10: Medical conditions and telemedicine ${ }^{170}$

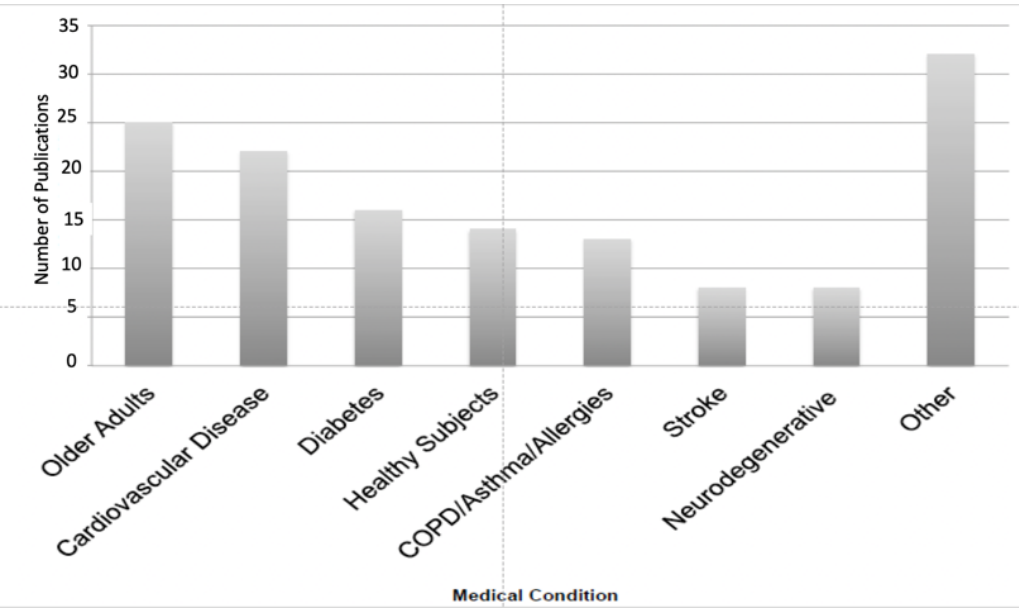

Online communities are another form of digital health may empower PwPD to be more active participants in their own care. ${ }^{172}$ Online health communities are a form of communication technology that allow patients to interact with their team of healthcare providers, other patients with the same diagnosis, and 
caregivers. These communities also allow patients to track their health information and become involved in research. ${ }^{182}$

\section{Telehealth}

There have been successful telehealth interventions in PwPD across various disciplines. Dorsey et al. ${ }^{156}$ conducted a 7-month, trial in which PwPD were randomly assigned to receive specialty care from a neurologist in-person or home via telehealth and found telehealth to be as effective as in personcare. Study findings indicate feasibility of telehealth for specialty visits, with a $93 \%$ completion rate of visits. ${ }^{156}$ Constantinescu et al. ${ }^{142}$ conducted an intervention where PwPD were randomized to receive voice treatment therapy via videoconference or in person and found that both groups saw an increase in acoustic measures and reported high patient satisfaction. Findings from this study confirmed the efficacy, validity and reliability of videoconference for voice treatment. ${ }^{142}$ Additionally, there was no difference between quality of life scores and motor scores between groups. ${ }^{156}$ Another randomized trial found telerehabilitation could effectively assess ADLs and hand function compared to an in-person assessment. ${ }^{183}$ Whether a comprehensive nutrition assessment can also be conducted via telemedicine has yet to be explored.

Two studies support using telemedicine as part of patient care but not in place of in-person care. Sekimoto et al. ${ }^{184}$ conducted a randomized crossover pilot study of telemedicine via iPads using Facetime. During the telemedicine visit, clinicians performed perform a structured interview, medication review and motor examination in between in-person visits. 
Findings from this study indicate telemedicine service could be used in conjunction with in-person visits, as no difference in quality of life was reported between groups and PwPD in the telemedicine group reported high satisfaction. Finally, Wilkinson et al. ${ }^{163}$ examined if telehealth delivered in the home and at a satellite clinic compared to those receiving usual-care and found no difference in patient satisfaction or clinical outcomes between groups. Those in the telehealth group saw a significant decrease in travel time, but compared to the usual-care groups the satellite arm had significantly lower proportion of "no-shows"/cancellations. ${ }^{163}$ Findings reiterate telehealth can enhance usual care health services and can benefit patients who face barriers to receive in-person care regularly. ${ }^{185}$

\section{Devices, Wearables, \& Sensors}

Over the last decade, advancements in sensors and wearable technologies have been made, allowing these innovative mediums to gain popularity to complete and compliment evaluations of PwPD. ${ }^{186}$ Devices such as Kinesia $^{\mathrm{TM}}$ and Parkinson's KinetiGraph (PKG ${ }^{\mathrm{TM}}$ ) can help detect bradykinesia, record resting and postural tremors, and differentiate patterns of bradykinesia and dyskinesia in the "on" and "off states." 186 Other wearables, such as accelerometers, gyroscopes (device used for measuring or maintaining orientation and angular velocity), and magnetometers (a noninvasive device that monitors heat function), can be integrated in garments or accessories. ${ }^{187}$ These accessories can also be used in conjunction with webbased applications. 
People with PD report added value of wearable devices. Ozanne et al. ${ }^{187}$ conducted focus groups among PwPD and found that participants saw the potential for wearable sensors to improve treatment, and believed the benefits outweighed the inconvenience of wearing sensors. When examining facilitators and barriers to utilizing sensors, participants indicated that userfriendly and a simple design interface would promote usability, as well as receiving interactive information from providers. ${ }^{187}$ However, participants were concerned about unclear information, inconclusive recordings and had concerns about protecting personal health information and integrity. In order to encourage use of sensors, findings indicate PwPD need to feel well-informed and find added value when using sensors. ${ }^{187}$ Wearables also need to be easy to use, have an attractive design and be efficacious for improving disease management. ${ }^{187}$ Findings further support the use of digital health to help manage track Parkinson's disease.

Devices, such as smart phones or iPads are becoming popular mediums to deliver health services to PwPD. mHealth, or "the use of mobile and wireless devices to improve health outcomes, healthcare services and research," has been effectively used in PD management. One study found peer coaching through mHealth can promote physical activity among PwPD and is safe, feasible and acceptable among participants. ${ }^{188}$ Both peer coaches and PwPD were satisfied or very satisfied with the program and all PwPD participating saw an increase average steps per day. ${ }^{188}$ These findings are significant because delivering support by telephone may be a viable way 
to increase physical activity and promote other healthy lifestyle patterns among PwPD. In a pilot study, Arrora and colleagues ${ }^{189}$ demonstrated the ability of consumer-grade smart phones to accurately differentiate PwPD from age-matched controls through effectively measuring symptoms. Findings from this study reveal the potential for mHealth to evaluate disease severity. ${ }^{189}$

The integration of wearable technology with smart devices can enable remote monitoring of PwPD and provide clinicians, caregivers and patients with real-time feedback. ${ }^{26}$ Patel et al. ${ }^{190}$ found that combining wearable sensors with a web-based application for home monitoring PwPD can provide reliable quantitative information that can inform clinical decisions. The system these researchers developed includes 8 accelerometers on the upper and lower limbs which is relayed to a mobile device or computer. The system promotes patient-clinician interaction through video-conferencing or real time access to the sensor data. ${ }^{190}$ The proposed system can successfully gather data from PwPD to inform symptom severity and motor fluctuations in between medication doses. Findings from this work indicate that this home monitoring system has the potential to simplify the process of monitoring medication effectiveness. ${ }^{190}$

\section{Online Health Communities}

Online support groups via forums, blogs and social media are becoming popular sources for health information. ${ }^{191}$ Over $50 \%$ of American adults living with a chronic disease have looked online for health information. ${ }^{192}$ It is estimated that 90 million (84\%) Americans have participated in online 
communities and that $43 \%$ of internet users are involved in online groups to help manage career, medical conditions or parenting. ${ }^{152}$ Approximately, $12 \%$ internet using adults with one or more chronic health conditions (e.g., cancer, fibromyalgia, non-specified chronic pain conditions, diabetes, Addison's disease, bipolar disorder, celiac disease, trigeminal neuralgia, Parkinson's disease, systemic lupus erythematosus (SLE), rheumatoid arthritis, Sjögren's syndrome, schizophrenia, cystic fibrosis, and cerebral palsy) maintain a blog and $28 \%$ read blogs about their condition written by others (Figure 11). ${ }^{192} \mathrm{~A}$ 2019 report examining social media use among Americans revealed $68 \%$ of Americans between $50-64$ years of age, and $46 \% 65+$ report using Facebook. ${ }^{193}$

Figure 11: Internet Activities Among Those With and Without Chronic Conditionsn ${ }^{192}$

\begin{tabular}{|c|c|c|c|}
\hline \multicolumn{4}{|l|}{ Internet activities } \\
\hline \multicolumn{4}{|c|}{ What internet users reporting $2+, 1+$, and no chronic conditions do online } \\
\hline & No conditions & $1+$ conditions & $2+$ conditions \\
\hline Send or read email & $90 \%$ & $90 \%$ & $91 \%$ \\
\hline Bank online & $58^{*}$ & 46 & 45 \\
\hline Look for info on Wikipedia & $51^{*}$ & 38 & 31 \\
\hline Get financial information & $40^{*}$ & 32 & 28 \\
\hline Use social network site & $39^{*}$ & 25 & 19 \\
\hline Read someone else's blog & $34^{*}$ & 28 & 26 \\
\hline Use Twitter or another service & $12^{*}$ & 7 & 7 \\
\hline Buy or sell stocks, mutual funds, or bonds online & $12^{*}$ & 9 & 5 \\
\hline Create or work on your own blog & 11 & 12 & 11 \\
\hline Participate in an online discussion & 7 & 8 & 8 \\
\hline \multicolumn{4}{|c|}{$\begin{array}{l}\text { * indicates a significant difference between those without chronic conditions and those with chronic } \\
\text { conditions. }\end{array}$} \\
\hline \multicolumn{4}{|c|}{$\sim$ Twitter and social network site use have grown since this survey was fielded. } \\
\hline \multicolumn{4}{|c|}{$\begin{array}{l}\text { Source: Pew Internet \& American Life Project Survey, November-December 2008. N=1650. Interviews } \\
\text { conducted in English or Spanish. Margin of error is } \pm 8 \% \text {. }\end{array}$} \\
\hline Pew Internet & & & \\
\hline
\end{tabular}


Online discussion forums are one of the most popular ways people interact with each other online. In a discussion forum, one person writes a message/post that can be answered by other members, which forms a conversation thread. ${ }^{194}$ Attard et al. ${ }^{191}$ examined positive and negative aspects of online PD supports groups through analyzing forum posts from four different support groups; membership within each group ranging from 1000 to 100,000. Benefits of peer communication appeared to be: exchange of knowledge between participants, formation of friendships and the development of support systems to cope with living with PD. The results revealed that participation in forums allowed patients to share experiences and knowledge, form friendships and help each other cope with the challenges of living with PD. ${ }^{191}$

While less frequently reported, there were some drawbacks to participating in online, peer-to-peer communication through forum posts. ${ }^{191}$ First, there are a lack of replies or lag time in between replies, ${ }^{191}$ which can hinder the experience. Findings revealed that tremors and cognitive impairments can hinder engagement in forum posting among PwPD. For instance, tremors would hinder one's their ability to answer posts when desired. ${ }^{191}$ Another barrier of online forum posting is that many PwPD did not want share personal information, as a result personal connections could not be formed between participants. For some participants, online support was not enough, and they felt lonely in their offline environment. Finally, misunderstanding and disagreements occurred on the forums due to lack of non-verbal cues. Findings from this study indicate online communities can 
offer PwPD emotional support and foster knowledge but should be used alongside traditional forms of support. ${ }^{191}$ Additionally, health professionals should partake in these online support groups through monitoring or facilitating posts to ensure the accuracy of information exchanged. ${ }^{191}$ Health professionals may also help guide PwPD using such online communities to reputable websites and publications. ${ }^{191}$ Roles of healthcare professionals in online communities is becoming especially important with the rise of online blogs.

Illness blogs are a type of online blog, where patients freely describe their experience managing their condition overtime. ${ }^{195}$ Readers have an opportunity to respond to blog posts, and as readers become more involved in the blog, a community is created. ${ }^{195}$ Shapira and colleagues ${ }^{195}$ completed a thematic analysis of 78 illness blog authors with PD to explore medical illness concerns blog authors had and found these authors typically discussed diagnosis and symptoms, treatment, coping mechanisms and providing information to their readers about PD. Treatments discussed included: medication, exercise, supplements, nutrition, complimentary therapies and surgeries. Only three authors blogged about nutritional modifications for PD, which included experimenting with diets to optimize medication management, trying vegan diets, fasting, and lowering sugar and protein intake. ${ }^{195}$ The reason that so few PwPD discussed diet in blog posts could be because nutrition is often excluded from the PD care plan. ${ }^{41}$ However, findings from this study reveal analyzing illness blogs may be a viable way understand the 
health concerns and medical and nutrition issues raised by PwPD online and in a non-medical setting. ${ }^{195}$

Personal online health communities have also been developed to promote online communication between PwPD and their healthcare providers. ${ }^{172}$ Visser et al. ${ }^{172}$ conducted semi-structured interviews and observed the use of online health communities among PwPD for over a year. Patients could communicate with their provider through diary entries or virtual meetings/postings. Providers did not receive notifications when the PwPD posted a diary entry but did with the virtual meeting post. Participants could also update a stored list of health problems and upload health documents.

Three major themes that emerged from these analyses were: "number of postings," "coming across as a complainer," and "hesitating about legitimacy of knowledge." In regard to "the number of postings," many PwPD expressed concerns about burdening their healthcare providers with too many questions and limited their number of postings to only concerns that needed immediate attention from their provider. However, PwPD found the diary section of the online health community to be less disruptive to healthcare providers and shared more information in their section of the community. ${ }^{172}$ The PwPD also felt obligated to report and update symptoms, but did not want to come across as a complainer, so refrained from expressing emotions or feelings regarding PD sequelae. ${ }^{172}$ The use of online communities can change the powerdynamics between the provider and PwPD, especially when the provider can no longer visually assess the PwPD and must depend on self-reported 
symptoms from the PwPD. ${ }^{172}$ Many PwPD believed the provider still possessed the greatest amount expertise about their condition, despite the change in delivery of care. ${ }^{172}$ As a result, PwPD presented their knowledge and interpretation of their symptoms as an opinion and were hesitant to make claims about their condition.

Findings from this study reveal even though online communities are innovative mediums to deliver healthcare services they have not met the expectation of creating more pro-active patients or changing the power processes between provider and patient and pre-existing norms regarding patient-provider dynamics persist. Future health communities need to focus on creating a two-way exchange between provider and patient, where healthcare providers share additional resources for managing PD to help PwPD gain more knowledge about the disease. More research also needs to assess digital health to manage the nutritional status and diet intake for PwPD or their caregivers. This is because PwPD are likely at nutrition risk and as nutrition concerns increase as PD progresses, caregiver responsibility to manage diet increases, but nutrition is often excluded from the treatment plan of managing PD. ${ }^{41}$

\section{A. Efficacy of Digital Health for Managing Nutrition}

Research has not directly examined the use of digital health for managing nutrition and $\mathrm{PD}$, but success with digital health for $\mathrm{PD}$, as well as digital health's ability to help manage nutrition in other populations ${ }^{196,197}$, compels expanded use of digital health nutrition services for PwPD. Digital 
health services can increase fruit and vegetable consumption ${ }^{196,197}$, decrease fat intake ${ }^{198}$, self-efficacy to make healthy dietary choices ${ }^{197}$, and promote sustainable dietary changes. ${ }^{198}$ Tailored digital nutrition interventions addressing participants' environment and learning preferences improved diet quality more than providing generic nutrition information through online modules $(n=1349) .{ }^{199}$

Mobile devices can help track dietary intake. ${ }^{200,201}$ Using mobile applications (e.g., Lose It! and MyFitnessPal) to track food intake can provide instant feedback about all calories and nutrients consumed. Dietary selfmonitoring however takes time and effort and technological advances such as Remote Food Photography Method are being developed to improve the ease of self-tracking dietary intake. ${ }^{200}$ Through the Remote Food Photography Method users submit photos pre and after consuming food to researchers and researchers perform semiautomatic computer analysis to determine the nutritional value of those foods. ${ }^{200}$ Neriah and Gelibeter ${ }^{201}$ conducted a retrospective cohort study to determine the effectiveness of using photography to track food intake via a smartphone weight loss application. Compared to the traditional weight loss app group the group with the photography feature lost significantly more weight and tracked their food for a longer duration. Weight loss in the photography group was mediated by the duration of app use and number of logged days in the program.

Telenutrition is another solution for PwPD and can help to increase access to nutrition information in rural areas. ${ }^{202}$ Telenutrition is the remote 
delivery of medical nutrition therapy (MNT) by a registered dietitian using interactive electronic information and telecommunication technology (e.g. videoconferencing). ${ }^{202} \mathrm{~A}$ randomized controlled trial assessing the feasibility of telenutrition weight loss intervention in middle-aged and older men with cardiovascular risk factors, found telenutrition services demonstrated patient satisfaction as well as good adherence and retention rates. ${ }^{203}$ During this $12-$ week intervention, men were randomized to either the intervention group or enhanced usual care group. The intervention group received three medical nutrition therapy sessions from a registered dietitian at week one, five, and nine to nine nutrition coaching sessions at weeks $2-4,6-8$ and $10-12 .{ }^{203}$ The MNT sessions included individualized nutrition assessment, education and counseling sessions. The coaching sessions were patient-led discussions around topics such as, self-monitoring weekly weight, goal setting, and overcoming barriers to dietary adherence. The enhanced usual care group did not receive nutrition coaching in between their in-person sessions at weeks one, six and $12 .{ }^{203}$

Both groups lost a significant amount of weight and there was no difference in the amount of weight loss in between groups. Both groups saw an improvement in total fruit $(p=0.05)$, whole grains $(p=0.004)$, and fatty acid ratio scores $(p=0.002)$. Participants lowered refined grain $(p=0.04)$, sodium $(p=0.01)$, added sugars $(p=0.01)$ and saturated fat scores $(p=0.002)$ healthy eating index (HEI) component scores. However, the intervention groups saw a greater improvement in total fruit $(p=0.04)$, whole fruit $(p=0.04)$, and green 
beans $(p=0.013)$. Findings from this study show promise for PwPD, as there is a higher occurrence of PD among men than women. ${ }^{12,13}$ More information is also needed to determine how digital health nutrition interventions may impact caregivers and reduce caregiver burden, as caregivers are involved in managing dietary intake for PwPD. ${ }^{24}$

\section{B. Efficacy of Digital Health for Informal Caregivers}

Technology, including digital health can support caregivers and promote better coping. ${ }^{204}$ More than 30 million US adults provide home caregiving and approximately $80 \%$ of these caregivers seek health information online.$^{205,206}$ Caregivers believe that technology can help provide more efficient, effective and safer care and reduce stress when delivering care..$^{204}$ Nearly $75 \%$ of caregivers stated they would be willing to pay for access to a website for a complex care matter and $85 \%$ believe insurance should pay for access to such websites. ${ }^{207}$ While digital health has been used to manage chronic disease more information is needed to understand the impact of telehealth interventions can have on informal caregivers and how digital health can offer support and address informal caregivers' health needs. ${ }^{204}$

Chi and Demiris ${ }^{204}$ conducted a systematic review assessing of 65 studies that included informal caregivers and utilized digital health technologies to provide education, consultations, psychosocial therapy, social support, data collection and monitoring systems, or deliver clinical services. The most common medium used by studies reviewed was videoconferencing. In $95 \%$ of the articles reviewed, caregivers reported improvements in 
outcomes, such as psychological health, satisfaction and comfort with digital health, caregiving knowledge and skill, and social support. ${ }^{204}$ Nearly a quarter of the studies reviewed, were conducted among caregivers living in remote or rural areas and brought significant improvements in caregivers' QOL and psychological well-being. Findings from this review conclude telehealth can provide acceptable care and save travel costs for caregivers of patients who need long-term care and monitoring, such as PwPD. ${ }^{204}$

Several studies have specifically examined digital health interventions delivered for caregivers of PwPD. ${ }^{176,208}$ These studies specifically looked at virtual support groups and their impact on coping with caregiving responsibilities, disease burden and QOL. ${ }^{176,208}$ In a study conducted by Marziali et al. ${ }^{208}$, caregivers of persons with Alzheimer's, Stroke or PD met weekly for 10 weeks via videoconference in small groups lead by a support group leader. At the end of 10 weeks and six-month follow up, $90 \%$ of participants reported a positive experience and found the group helped with coping with the stresses of caregiving. Findings revealed virtual support groups were comparable to in-person groups. In a small pilot study, caregiver tele-support groups was a feasible option and showed promise to potentially improve depression scores and decrease caregiver burden. ${ }^{176}$ Whether nutrition assessment and nutrition interventions can also be delivered to caregivers of PwPD warrants exploration.

\section{Nutrition Assessment}


Poor nutritional status is an imbalance of energy and/or nutrient intake, resulting in suboptimal body weight and composition, function, and/or clinical outcomes, which can eventually lead to malnutrition in the forms of over- and under- nnutrition. ${ }^{209,210}$ Assessing the nutritional status of PwPD includes the traditional examination of dietary intake, anthropometrics, and biochemical and clinical measures, ${ }^{209,210}$ as well as a focus on possible food-drug interactions, duration of medications and history of depression and anxiety. ${ }^{70} \mathrm{~A}$ crosssectional study, examining nutritional intake of PwPD for nutritional and protein risk, found that nearly $63 \%$ of PwPD would be categorized as with malnutrition or at risk for malnutrition, $\sim 53 \%$ exhibited weight loss in the last three months but presented with elevated waist circumference..$^{211}$

\section{A. Dietary Intake and Diet Quality}

Disease sequalae, physiological factors and treatments for PD can compromise dietary intake and quality. ${ }^{61}$ Disease stage and side -effects from medication or surgery can impact dietary choices by impacting appetite and the ability to consume food. ${ }^{212}$ Other factors that related to PD that can impact dietary choices include: nausea, delayed gastric motility, dehydration, constipation, change in taste and smell, and dysphagia. ${ }^{61}$ Complaints relate to change in taste and smell are among the most frequently reported from PwPD. One study found $26 \%$ of PwPD complained of taste/smell issues, compared to only $7 \%$ of controls. ${ }^{213}$ How change in taste and smell impact nutritional status and diet quality has not been directly explore. Cognitive 
decline and depression, common among PwPD, can also compromise food intake by impacting mealtime and appetite ${ }^{214}$

Dysphagia can also impact mealtime and the amount consumed as it results in change in dietary choices and avoidance of certain foods, which can impact weight status. Those with dysphagia are more likely to experience unintentional weight loss, especially PwPD avoiding solid foods due to swallowing issues. ${ }^{214}$ A longitudinal prospective study, examining dietary intake, weight, and swallow function among, found that PwPD with weight loss were more likely to avoid solid foods due to swallowing difficulties compared to controls. ${ }^{214}$ Those PwPD who experienced weight loss also consumed less vegetables, fresh fruit, meat on sandwiches and drinks without energy at the first evaluation. ${ }^{214}$ However, whether this weight loss was associated with swallowing difficulties was not explored. Avoidance of solid food and fluid can result in nutritional imbalances and hydration. Variability in weight and nutritional status is a concern because it can impact diet quality as well as physical functioning, which can lead to muscle wasting and difficulties in performing ADL. ${ }^{215}$

Though low dietary quality scores are associated with chronic disease such as cardiovascular disease ${ }^{216}$, few studies have examined diet quality and intake in PwPD. LoBuono et al. ${ }^{38}$ found low dietary quality scores among a small cohort of PwPD. Cassani and colleagues ${ }^{217}$, further examined dietary intake among PwPD and healthy controls, specifically regarding the adherence to a Mediterranean diet, and found PwPD ate more fruit, cooked 
vegetables, cereals and baked items, as well as more sweets and dressings. ${ }^{217}$ Those with PD also consumed less fish and alcohol and drank significantly less water, coffee or milk, ${ }^{217}$ and as a result, consumed significantly less fluid overall. ${ }^{217}$ Overall PwPD had higher intake of calories, iron, zinc, folate, and vitamins $\mathrm{A}$, and $\mathrm{C}$, however PwPD also had significantly lower BMls compared to controls $\left(26.2 \pm 4.9\right.$ vs. $\left.28.5 \pm 6.4 \mathrm{~kg} / \mathrm{m}^{2}, \mathrm{p}<0.001\right) .{ }^{217}$ Dysphagia was self-reported among $12 \%$ which resulted in a significant decrease in fluid intake and preference for more vicious foods, but did not impact adherence to the Mediterranean Diet. There are two major areas of significance with these findings. First, the difference in dietary intake between PwPD nad control warrants a similar exploration between PwPD and their informal caregivers. Also, a prospective, longitudinal examination of how dietary intake and quality relate to disease progression. ${ }^{217}$

Protein intake among PwPD is also a concern for those taking levodopa. Dietary protein and levodopa compete for absorption and transport across the gut, blood-brain barrier, and peripheral nervous system. ${ }^{218}$ It is advised to wait 30 to 60 minutes between taking levodopa and eating a high protein meal. ${ }^{211}$ Participants taking levodopa exceeded daily protein recommendations, consuming $1.4 \pm 0.6$ per day, rather than the 0.8 grams/kg/day. ${ }^{211}$ Seventy-five percent of PwPD ingested levodopa with food. ${ }^{211}$ Findings highlight the need for nutrition education on protein timing and medication. 


\section{B. Impact on Weight and Body Composition}

Weight and nutritional status in PwPD varies during disease progression (Figure 12$)^{61}$ and can adversely affect body composition, cognitive and physical functioning ${ }^{144,214,219}, \mathrm{QOL}$ and health outcomes. ${ }^{220} \mathrm{~A}$ decade before diagnosis, an average 5.2 pound weight loss is reported, despite increased energy intake. ${ }^{221}$ Much of this weight loss is attributed to the increase in energy expenditure caused by untreated motor symptoms. ${ }^{221}$ Once treatment is initiated symptoms are minimized, and weight gain is observed ${ }^{61,222,223}$

Figure 12: Nutritional Status Over the Course of $\mathrm{PD}^{61}$

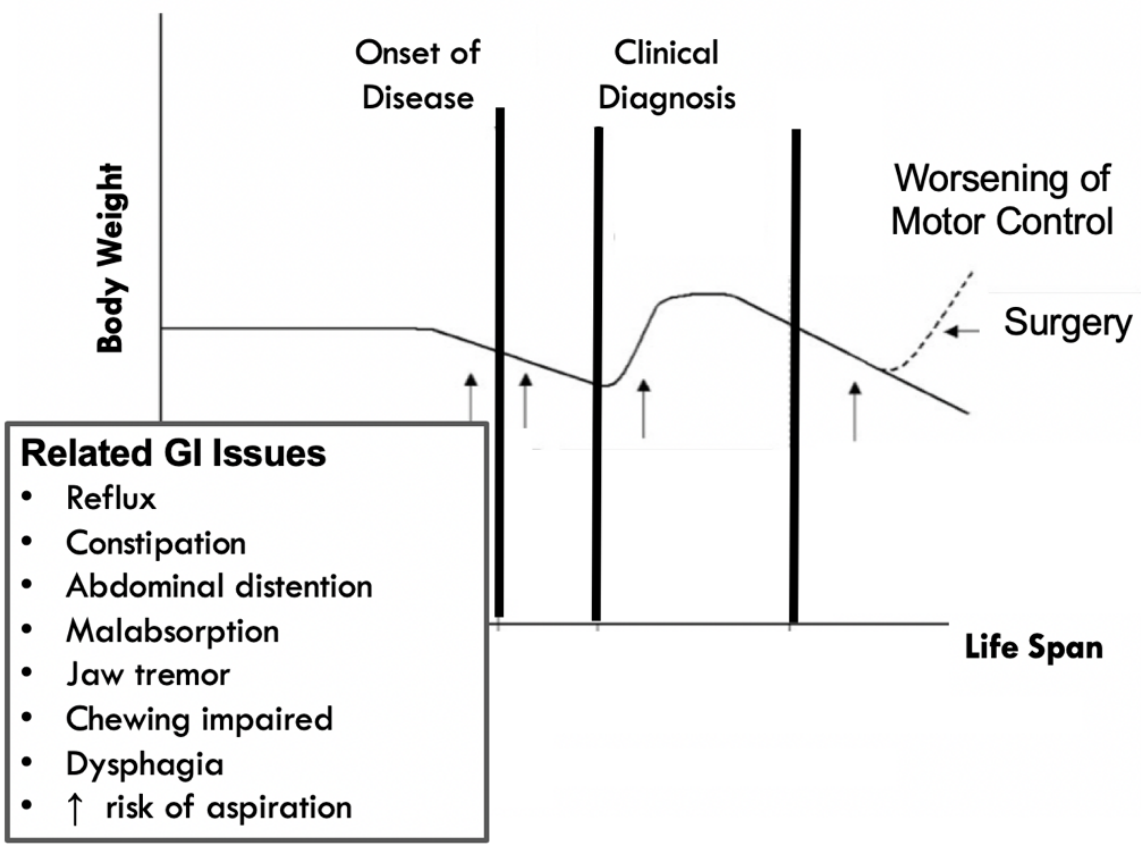

Weight status changes throughout the course of PD (Figure 12), at the beginning of treatment for PD excess weight is a problem, but as the disease progresses, underweight and nutrition results. ${ }^{61}$ Weight gain among PwPD 
can result in overnutrition 224 caused by excessive oral intake and/or inadequate activity. ${ }^{225}$ One study found a majority of PwPD and their spouses are overweight or obese ${ }^{226}$, indicating the need for early nutrition intervention for PwPD and caregivers to reduce chronic disease risk. ${ }^{227}$ Side effects from some PD medications, such as dopamine agonists, can increase impulsivity, lead to overeating and result in weight again. ${ }^{227}$ Additionally, treatments can reduce dyskinesia, which can decrease energy expended and result in weight gain. ${ }^{61}$ Furthermore, anxiety and depression are common in PwPD and prescribed medications can cause weight gain. ${ }^{228,229}$ Following the implantation of deep brain stimulation (DBS), a mean weight gain of 3.1 to 9.3 $\mathrm{kg}$ within three months has been reported, and typically curtails about a year after implantation. ${ }^{227,230}$ Weight gain among PwPD first receiving DBS is attributed to reduced energy expenditure since dyskinesias are reduced after DBS. ${ }^{168}$ Being overweight increases risk of developing sarcopenic-obesity, higher body fat and decreased muscle strength that can exacerbate a deterioration in physical function. ${ }^{215}$

Due to disease-related decline in mobility, it is speculated the age-related loss of skeletal muscle mass and muscle strength ${ }^{231}$ are prevalent in PwPD and impact the ability to perform ADLs, increase fall and fracture risk, and compromise QOL. ${ }^{232}$ People with PD often weigh less than healthy controls, but have similar amounts of abdominal fat ${ }^{20}$ and a higher proportion of visceral to subcutaneous fat ${ }^{233}$, excess visceral fat is linked with increased risk for chronic disease. ${ }^{233}$ For PwPD, presence of central obesity is a risk factor for 
cognitive impairment ${ }^{234}$, and changes in body fat distribution may be attributed to changes in macronutrient distribution. ${ }^{234}$ Conversely, other research indicates PwPD have a higher fat-free mass and possibly a more favorable body composition compared to healthy controls. ${ }^{235,236}$ Inconsistent findings related to diet and body composition warrants a deeper understanding of chronic disease in PwPD, such as cardiometabolic. ${ }^{237}$

A study assessing weight stability of PwPD over one year found a significant amount of muscle converted to fat. ${ }^{224}$ However there was no association between nutritional variables (BMI, weight, Seniors in the Community: Risk Evaluation for Eating and Nutrition version II questionnaire, mid-upper arm circumference, hand grip strength, triceps skin-fold) of interest and motor and non-motor features of PD. Findings from this study warrant longer observational studies to track nutrition outcome variables overtime to better understand the relationship between nutrition outcome variables and motor and non-motor sequelae. Findings from this study also indicate malnutrition should be screened regularly among PwPD to help identify those at risk of muscle loss and decrease mobility, and that nutrition professionals should be included in the care plan of PD.

As PD progresses, levodopa tolerance develops, sequelae worsen, and weight loss and under-nutrition are common. ${ }^{61}$ Undernutrition results from inadequate intake to meet energy expenditure. ${ }^{225} \mathrm{Up}$ to $25.5 \%$ of PwPD are malnourished and up to $26.5 \%$ are at risk for malnutrition. ${ }^{70,220,238}$ After PD diagnosis, PwPD weight can fluctuate up or down and very few maintain their 
body weight. One study found, despite increased energy intake, the average weight loss in PwPD ( $n=174)$ was 7.7 pounds 8 years following diagnosis. ${ }^{239}$ Significant weight loss is experienced among both men and women. ${ }^{240}$ Unintentional weight loss can be caused by a variety of factors. ${ }^{70}$ Mobility limitations make it challenging to buy, prepare, and cook food, ${ }^{61}$ and PwPD may exhibit dysphagia and gastrointestinal problems, which can diminish appetite. ${ }^{212}$ Higher levodopa dosages are associated with a lower BMI, ${ }^{229}$ which is associated with compromised motor and non-motor function. ${ }^{219}$ Weight loss among PwPD is negatively associated with cognition, suggesting an association between cognition and nutritional status. ${ }^{214}$ Weight loss is also exhibited among PwPD experiencing dementia or visual hallucinations. ${ }^{241}$ Whether PwPD experience Despite under- or over-nutrition, malnutrition in PwPD is under-treated. ${ }^{144,242}$ The variability in weight status, cognition, and physical functioning warrants innovative nutrition care to improve health outcomes..$^{38}$ Caregivers should be part of/included when making care/treatment plans for PwPD whose responsibilities increase as PD advances ${ }^{25}$, in turn compromising the health of the caregivers. ${ }^{29}$ Fluctuations in nutrition and weight status compromises body composition and can impact cognitive and physical functioning ${ }^{144,214,219} \mathrm{~A}$ decrease in fat-free mass and an increase in fat mass is a natural part of aging and can accelerate decline in physical function ${ }^{215}$, however the added effect PD has on age-related muscle loss is unclear. ${ }^{144,235}$ How this fat free mass increase impacts cardiometabolic status among PwPD also needs greater exploration. 


\section{Cardiometabolic Risk in PD}

Inconsistent research exists regarding cardiometabolic risk among PwPD. In a small cross sectional study, six of seven participants had at least one sub-optimal cardiometabolic lab value and two PwPD were on statinlowering drugs. ${ }^{38}$ Chahine et $\mathrm{al}^{243}$ examined vascular risk factors (diabetes, hypertension, obesity) among early PD and found an association between the presence of vascular risk and concentration of white matter hyperintensities (WMH). Greater presence of WMH was predictive of decline in verbal memory two years later. ${ }^{243}$ Cardiometabolic and vascular risk factors are associated with cognitive decline ${ }^{244}$ Research needs to exam how vascular risk factors can impact cognition beyond two years needs to be assessed.

Previous research suggests a cardiometabolic protective effect of PD and theorizes that optimal HDL-C levels among PwPD may explain this theory. ${ }^{237}$ In a larger cross-sectional study $(n=150)$, despite excess visceral fat or poor nutritional status, longer PD duration was associated with optimal high-density lipoprotein (HDL) cholesterol levels, indicating possible cardiometabolic protective properties of PD. ${ }^{237}$ Cereda et al ${ }^{236}$ also investigated the cardiometabolic status among PD and found compared to healthy controls, PwPD exhibited a lower percentage of body fat and moreoptimal glucose levels and lipid profiles. However, there was no difference in waist circumference between PwPD and healthy controls. ${ }^{236}$ Wei et al ${ }^{245}$ performed a retrospective study comparing serum lipid and lipoprotein levels among PwPD ( $n=110)$, controls $(n=130)$, persons with intracerebral 
hemorrhage $(n=140)$ and with an acute cerebral infarction $(n=140)$; findings revealed PwPD had reduced serum levels of triglycerides, apolipoprotein B and very low density lipoprotein (VLDL) cholesterol. ${ }^{245}$ Further research is needed to explore the relationship between nutrition and the changes of lipids and lipoproteins among PwPD. ${ }^{245}$ Additionally, how this protective cardiometabolic profile may impact health outcomes overtimes warrants further investigation..$^{236}$

\section{Nutrition Recommendations}

Protein, water, fiber, vitamin $D$, and omega-3 fatty acids are nutrients of concern for PwPD due to sequelae and medications.${ }^{61}$ Over-time, patients with higher dietary protein intake may require higher levodopa doses and eventually the medication loses effectiveness. ${ }^{20}$ Common gastrointestinal sequelae include delayed gastric emptying and constipation and warrant the need to manage daily water and fiber intake. ${ }^{246}$ Reported low vitamin $D$ status in PwPD can impact bone health and increase risk of fracture with diseaserelated physical decline. ${ }^{247}$ Since PwPD are at increased risk for falls, helping this population to optimize bone health to prevent fracture is essential. ${ }^{41}$ In addition to neuroprotective effects ${ }^{52}$, omega-3 fatty acid can improve depressive symptoms in PwPD. ${ }^{248}$

Formal, comprehensive dietary guidelines for PwPD have not been established. ${ }^{249}$ This is partly due to the heterogeneity of PD and the need for individualized recommendations. There are multiple dietary recommendations for PwPD that focus on weight management, protein intake, and constipation ${ }^{20}$ 
and can be confusing for PwPD and their caregivers. Diets adequate in fiber and probiotics are proposed for early phase PD to improve gastrointestinal issues, increase absorption of levodopa ${ }^{250}$ and promote optimal cognitive function. ${ }^{61,222}$ Planted-based, vegetarian, or Mediterranean diets are proposed for early-phase PD prior to the introduction of levodopa containing medications because they are adequate in fiber and pre- and probiotics. These specific diets can improve gastrointestinal issues, increase absorption of levodopa ${ }^{20,61,251}$ and promote optimal cognitive function. ${ }^{61,222}$

National organizations for PD provide dietary suggestions which include: 25-35 grams of fiber per day, ${ }^{252}$ up to 70 fluid ounces of water ${ }^{252}$, and encourage consumption of nuts, tuna, salmon and dark green leafy vegetables to promote cognitive health. ${ }^{253}$ These organizations also advise to monitor vitamin D status. ${ }^{253}$ Supplementing with vitamin $E$ and omega-3 may also be beneficial. A trial found co-supplementing with $400 \mathrm{IU}$ of Vitamin and 1000 $\mathrm{mg} /$ day of omega-3 fatty acid from flax seed oil significantly improved overall PD clinical outcome scores, total antioxidant capacity, insulin metabolism. glutathione concentrations, and high-sensitivity C-reactive protein levels. ${ }^{254}$

Guidelines for managing dietary protein intake with levodopa also exist and must be based on the frequency and timing of taking levodopa. As a result, there is confusion among PwPD and their caregiver on how to optimize medication timing and dietary protein intake. ${ }^{108}$ Recommendations include: 1) a low protein diet; 2) a protein redistribution diet; 3) allowing thirty minutes to two hours before or after eating to take levodopa medication..$^{251,255} \mathrm{~A}$ low 
protein diet restricts protein intake to $<0.8 \mathrm{~g} / \mathrm{kg}$ of ideal weight $/$ day. ${ }^{256} \mathrm{~A}$ protein redistribution diet recommends consuming the recommended dietary allowance (RDA) of $0.8 \mathrm{~g} / \mathrm{kg}$ of protein/day to ensure adequate protein intake, but requires the majority of dietary protein to be consumed at dinner to reduce daytime motor symptoms and minimize interaction between medication and protein. ${ }^{251}$ Cereda et al ${ }^{251}$ completed a systematic review examining the effectiveness of the low protein and protein redistribution diets, and found evidence supports the safety and efficacy of a protein redistribution diet.

Among PwPD there seems to be low adherence to protein the redistribution diet and low protein diets due to low palpability and acceptability as well as logistic difficulties for adopting the diet. ${ }^{223}$ As a result, waiting an 30 minutes to two hours for meals is encouraged by most PD organizations. ${ }^{223}$ More research is needed to understand long-term effects of the protein redistribution diet on nutritional status and to help participants find them more appealing and less burdensome. ${ }^{223}$ Increasing access to nutrition information through innovative mediums such as digital health will allow PwPD and caregivers to work with dietitians, pharmacists and physicians to manage medication side-effects and interactions.

\section{E. Nutrition and Quality of Life}

Nutritional status is an important component of QOL and should be incorporated in the care PwPD ${ }^{257}$, but is often an under-recognized component of care for PwPD. ${ }^{144}$ Under-nourished PwPD receiving general nutrition intervention or personalized dietary recommendations saw a significant 
improvement in emotional overall well-being. ${ }^{20}$ Many PwPD and their caregivers experience decline in psychosocial health, and support groups and group education can alleviate these feelings through promoting interactions with other PwPD and caregivers. ${ }^{75,258,259}$ Health promotion programs designed for PwPD increase overall wellbeing but also foster feelings of enjoyment and sense of social self. ${ }^{260}$ Access to transportation due to disease sequelae can be a barrier and increases caregiver burden. ${ }^{261}$ The observed variability in weight status, cognition, and physical functioning may warrant innovative nutrition care ${ }^{38}$, and should also include informal caregivers whose responsibilities increase as PD advances. ${ }^{25}$

\section{Emerging Middle Range Theory of Transitions}

Including caregivers in formative research targets elements of the emerging middle-range theory of transitions (Figure 13). ${ }^{262} \mathrm{~A}$ transition represents change from one state or condition to another, and includes life development stages, such becoming an informal caregiver. The theory promotes the exploration and understanding health and illness transition experiences, through understanding facilitators and barriers to a successful transition, and assessing the outcomes of successful transitions. ${ }^{148,262}$ Successful outcomes examined include: increase knowledge and skills that promote self-efficacy, coping, and satisfactory relationships with family, friends and formal services. ${ }^{262}$ The theory posits itself on helping informal caregivers to acquire new skills and knowledge to cope with situations related to caregiving, and creating feelings of informal support for caregivers, which play 
a central role in successful role transition. ${ }^{262}$ The theory has been

incorporated previously to guide the development of dyadic interventions for $\mathrm{PD}^{148}$ and Alzheimer' disease. ${ }^{263}$

Figure 13: Schematic of the Emerging Middle Range Theory of Transitions ${ }^{262}$

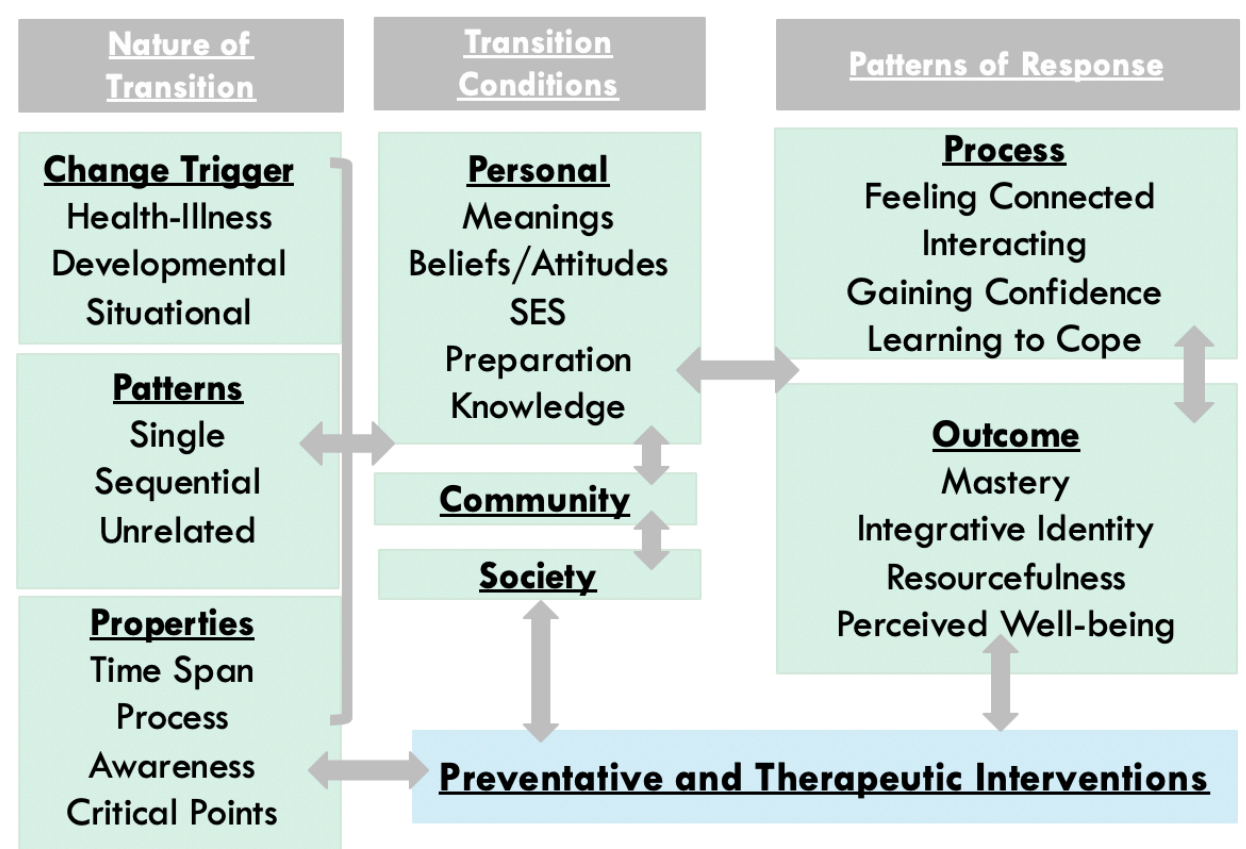

Research and services are beginning to identify the need for informal caregivers to help manage long-term conditions such as PD. ${ }^{137,264,265}$

Development of more comprehensive approaches to help PwPD and their caregivers adapt to changes they experience as a consequence of a long-term condition are warranted. ${ }^{265,266}$ Caregivers of PwPD are often faced with restricted work and social activities, increased worry and uncertainty about the future, loss of income, and feelings of guilt, frustration and/or grief. ${ }^{267,268}$ Consequently, PD caregivers are at risk for compromised psychosocial outcomes, which include poor QOL, emotional and financial strain, sleep disturbances, social isolation and increased risk of chronic illness. ${ }^{28,267}$ Non- 
motor sequelae among PwPD (e.g., depression, cognitive impairment) and depression among caregivers are predictive of caregiver burden. ${ }^{267}$ While social support may be a protective feature in minimizing caregiver burden. ${ }^{267}$ Whether caregiver burden is associated with poorer nutrition status for caregivers or their loved one with PwPD has yet to be explored.

As a result, future programs and services for PwPD should incorporate the preferences and needs of informal caregivers as their responsibility tends to increase as the disease progresses. ${ }^{266}$ Comprehensive, interdisciplinary services can help both PwPD and caregivers better cope and adjust with living with PD and improve QOL. ${ }^{265}$ Including PwPD and caregivers will be especially beneficial in digital health interventions and nutrition services as caregivers will likely have an active role in managing both of these as PD progresses and reliance on caregivers increase. Qualitative dyadic research among PwPD and caregivers, show concerns about keeping both parts of the dyad healthy. ${ }^{148}$ As a result, how the inclusion of caregivers can help promote healthy dietary patterns among PD Caregivers warrants exploration.

IV. Inclusion of Caregivers

Up to 6.5 million family caregivers provide substantial help, including coordination of care, medication management, and personal care for older adults living with disability. ${ }^{21}$ One quarter of informal caregivers provide nearly 45 hours of care per week for their loved with $\mathrm{PD},{ }^{22}$ and over a quarter spend more than 75 hours per week providing care. ${ }^{269}$ Additionally, caregivers commonly support PwPD at visits with clinicians, but are only engaged by 
physicians $40-70 \%$ of the time.$^{270}$ Compared to matched control caregivers, caregivers of PwPD exhibit higher direct and indirect costs consistently over five-years. ${ }^{271}$ When examining income progression over five-years, caregivers of PwPD also exhibited a higher cumulative income loss (Figure 14). ${ }^{271}$

Figure 14: Cumulative Income Loss for PD caregivers versus matched controls 271

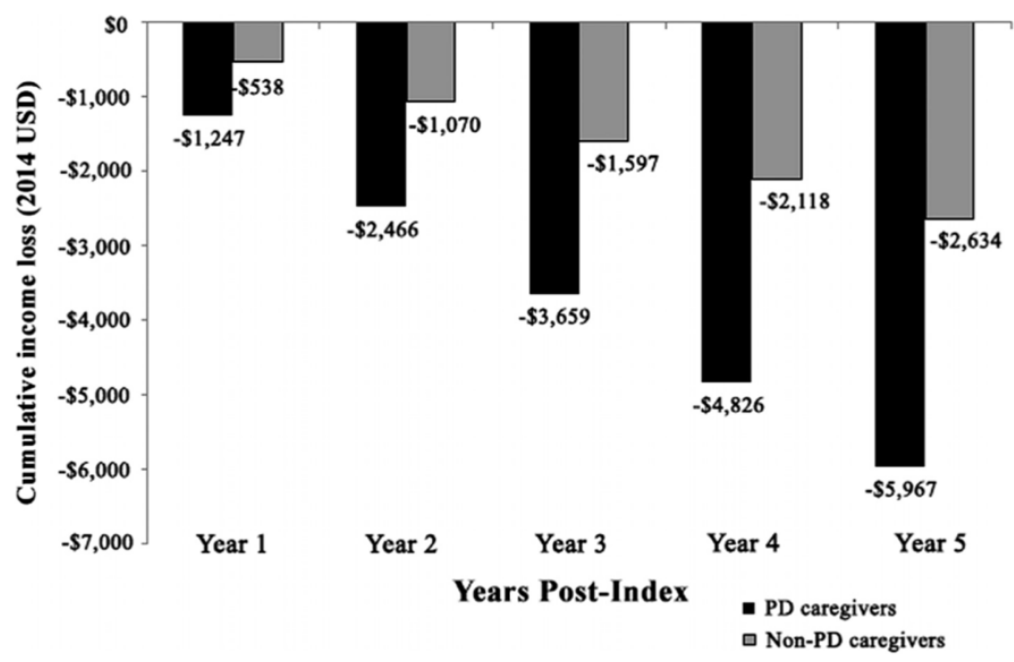

In addition to economic disadvantage, PD caregivers also experience stress and burden for caring with their loved one with PD. ${ }^{29}$ Male PD caregivers have decreased access to informal caregiving resources, despite reporting less strain than female caregivers. ${ }^{272}$ Women with PD receiving care from a male informal caregiver are more likely to use, formal, paid caregivers. ${ }^{272}$ Caregiving is a fundamental and valuable part of PD treatment but limited research has been conducted on caregiving patterns and needs for caring for a loved one with PD. ${ }^{272}$ In particular, dietary concerns, nutritionals status, and how technology can enhance dietary management caregiving has not been explored. To help include caregivers in the healthcare team, 
innovative mediums are necessary to increase access to caregiving and reduce disparities among PwPD. ${ }^{272}$

Caregivers experience primary stressors and secondary role strains as a result of providing care for their loved one.$^{56}$ Primary stressors include both subjective and objective difficulties directly related to providing care..$^{273}$ Examples of objective primary stressors include care tasks and the degree of impairment of the care recipient. ${ }^{273}$ Subjective primary stressors are the caregivers' emotional and psychological response to objective stressors. ${ }^{273}$ Primary stressors can negatively impact caregivers' physical and mental health. ${ }^{274}$ Secondary role strains are when hardships experienced in roles and activities that originate from primary stressors proliferate to other life areas. ${ }^{273}$ An example of secondary role strain is decreased participation in non-care activities or reluctance to partake in activities due to the time, energy and resources required to providing care. ${ }^{275}$ Older caregivers and those providing help with ADLs and healthcare management (e.g. scheduling appointments) are more likely to decrease participation in non-care activities. ${ }^{56}$

Caregiver activity restriction is also associated with poor health outcomes for the caregiver, such as reduced sleep quality and high blood pressure. ${ }^{276,277}$ Compared with matched controls caregivers, PD caregivers had higher rates of comorbidities, such as headache, gastroesophageal reflux disease, hypertension, and irritable bowel syndrome. ${ }^{271}$ Many of these diseases require dietary modifications to minimize and manage symptoms and suggest nutritional education may be beneficial. Further research is needed to assess 
the nutritional status and nutrition needs of PD caregivers. Additionally, given the later-onset of PD, most caregivers are older adults and have their own medical conditions. ${ }^{259}$

Informal caregivers for PwPD are at increased risk for poorer QOL, which is partly attributed to observing disease-related physical and cognitive decline in their loved ones. ${ }^{29}$ Informal caregivers are typically responsible for performing the majority of $A D L$, including buying and preparing food, and transportation. ${ }^{22}$ Caregiving stress can also resonate adverse consequences on the patient-caregiver dyad, compromising the care provided, as well as negatively impacting relations with the larger-family system. ${ }^{56}$

Studies have examined the lived experience of living with PD, needs and preferences of PD-Caregiver Dyads related to managing Parkinson's disease. ${ }^{278}$ Smith and Shaw ${ }^{279}$ exained the lived experience of both the PwPD and their caregiver within a family unit and found partners share the impact of PD, were learning to live in a new way to adopt to disease progression and felt a sense of belonging from support groups. Additional research can help identify services required to facilitate the process of learning to live with $P D,{ }^{279}$ such research should include how nutritional management can help facilitate this process. How dyads experience related to food and access nutrition information has yet to be explore.

Habermann \& Shin ${ }^{278}$ conducted a descriptive qualitative study among caregiver and PwPD to explore how dyads discuss needs, concerns and preferences for advanced PD. Findings indicated dyads wanted improved 
communication with healthcare providers and had concerns with choking, falls, voice production, financial strain and the future of disease progression. ${ }^{278}$ Increasing access to nutrition services can help address some of these concerns; nutrition professionals can provide education on optimizing dietary intake when modifying textures and calculating nutrition support needs to accommodate swallowing issues, as well as optimize dietary intake to promote a healthy body composition to prevent fall risk.

Educating caregivers early at disease diagnosis and encouraging caregivers to partner with healthcare providers can reduce stress and family conflict. ${ }^{135}$ Couple-oriented interventions improve spousal coping strategies, promote disease related stress and anxiety management, increase selfefficacy (one's belief in his/her capability to perform, organize, or execute a task or succeed in a situation ${ }^{280}$ ), and help couples manage changes caused by PD. ${ }^{32}$ Innovative mediums, such as digital health via video, web-based, telephone-based and remote-monitoring can have a significant improvement on caregiver outcomes. ${ }^{204}$ The use of technology can enhance caregiving experience and facilitate shared decision making, where patients and caregivers are actively involved in the care process and participate in the decision making processes. ${ }^{204}$

\section{Conclusion}

Due to limited mobility, transportation access, need for visual assessment and ongoing interdisciplinary care, PwPD are excellent candidates for digital health ${ }^{26,139}$, which may help increase opportunities for nutrition services. 
Understanding how the nutritional status of PwPD change overtime can help health care professionals better understand the nutritional needs of PwPD and provide effective nutrition services via digital health. Therefore, the purpose of this mixed-methods project is to: 1.) Understand how the nutritional status of PwPD changes overtime; 2.) examine the perceptions, acceptance, facilitators, and barriers for adopting digital health to improve the nutritional health of PwPD and their informal caregivers; 3.) understand the nutritional status and digital competence of PwPD and their informal caregivers. 


\section{Literature Cited:}

1. Fahn S. Description of Parkinson's disease as a clinical syndrome. Annals of the New York Academy of Sciences. 2003;991:1-14.

2. Borlongan CV, Burns J, Tajiri N, et al. Epidemiological survey-based formulae to approximate incidence and prevalence of neurological disorders in the United States: A meta-analysis. PloS one. 2013;8(10):e78490.

3. O'Brien JA, Ward A, Michels SL, Tzivelekis S, Brandt NJ. Economic burden associated with Parkinson disease. Drug Benefit Trends. 2009;21(6):179-190.

4. Kowal SL, Dall TM, Chakrabarti R, Storm MV, Jain A. The current and projectedd economic burden of Parkinson's disease in the United States. Mov Disorder. 2013;28(3):311-218.

5. Zhao YJ, Wee HL, Chan YH, et al. Progression of Parkinson's disease as evaluated by Hoehn and Yahr stage transition times. Movement disorders : official journal of the Movement Disorder Society. 2010;25(6):710-716.

6. Lees AJ, Hardy J, Revesz T. Parkinson's disease. Lancet. 2009;373(9680):2055-2066.

7. Cilia R, Cereda E, Klersy C, et al. Parkinson's disease beyond 20 years. $J$ Neurol Neurosurg Psychiatry. 2014;86(8):849-855.

8. Pringsheim T, Jette N, Frolkis A, Steeves TD. The prevalence of Parkinson's disease: a systematic review and meta-analysis. Movement disorders : official Journal of the Movement Disorder Society. 2014;29(13):1583-1590.

9. Nutt JG, Wooten GF. Clinical practice. Diagnosis and initial management of Parkinson's disease. The New England journal of medicine. 2005;353(10):1021-1027. 
10. Bear MF, Connors BW, Paradiso MA. Neuroscience: Exploring the Brain 3rd ed. Baltimore, MD Lippincott Williams \& Wilkins 2007.

11. Wright Willis A, Evanoff BA, Lian M, Criswell SR, Racette BA. Geographic and ethnic variation in Parkinson disease: A population-based study of US Medicare beneficiaries. Neuroepidemiology. 2010;34(3):143-151.

12. de Lau LM, Breteler MM. Epidemiology of Parkinson's disease. Lancet neurology. 2006;5(6):525-535.

13. Alves G, Forsaa EB, Pedersen KF, Dreetz Gjerstad M, Larsen JP. Epidemiology of Parkinson's disease. Journal of Neurology. 2008;255 Suppl 5:18-32.

14. Van Den Eeden SK, Tanner CM, Bernstein AL, et al. Incidence of Parkinson's disease: variation by age, gender, and race/ethnicity. American Journal of Epidemiology. 2003;157(11):1015-1022.

15. Hughes AJ, Daniel SE, Kilford L, Lees AJ. Accuracy of clinical diagnosis of idiopathic Parkinson's disease: a clinico-pathological study of 100 cases. $J$ Neurol Neurosurg Psychiatry. 1992;55(3):181-184.

16. Kotagal V. Is PIGD a legitimate motor subtype in Parkinson disease? Annals of Clinical and Translational Neurology. 2016;3(6):473-477.

17. Lewitt PA. Levodopa for the treatment of Parkinson's disease. The New England Journal of Medicine. 2008;359(23):2468-2476.

18. Schapira AH, Emre M, Jenner P, Poewe W. Levodopa in the treatment of Parkinson's disease. European Journal of Neurology. 2009;16(9):982-989.

19. Chou KL, Stacy M, Simuni T, et al. The spectrum of "off" in Parkinson's disease: What have we learned over 40 years? Parkinsonism \& related disorders. 2018;51:9-16. 
20. Barichella M, Cereda E, Cassani E, et al. Dietary habits and neurological features of Parkinson's disease patients: Implications for practice. Clinical nutrition. 2016.

21. Wolff JL, Spillman BC, Freedman VA, Kasper JD. A national profile of family and unpaid caregivers who assist older adults with health care activities. JAMA Internal Medicine. 2016;176(3):372-379.

22. The National Alliance for Caregiving (NAC) and the AARP Public Policy Institute. Caregiving in the US. 2015;

http://www.aarp.org/content/dam/aarp/ppi/2015/caregiving-in-the-unitedstates-2015-report-revised.pdf Accessed January, 2017.

23. Noureldin M, Murawski MM, Mason HL, Hyner GC, Plake KS. The association between family caregivers' involvement in managing older adults' medications and caregivers' information-seeking behavior. J Am Pharm Assoc 2003;67(2):170-177.

24. Karlstedt M, Fereshtehnejad SM, Aarsland D, Lokk J. Determinants of Dyadic Relationship and Its Psychosocial Impact in Patients with Parkinson's Disease and Their Spouses. Parkinson's disease. 2017;2017:4697052.

25. Leroi I, McDonald K, Pantula H, Harbishettar V. Cognitive impairment in Parkinson disease: Impact on quality of life, disability, and caregiver burden. Journal of Geriatric Psychiatry and Neurology. 2012;25(4):208214.

26. Espay AJ, Bonato P, Nahab FB, et al. Technology in Parkinson's disease: Challenges and opportunities. Movement Disorders. 2016;31(9):12721282.

27. Parkinson's Australia Inc. Living with Parkinson's Disease: An updated economic analysis 2014 Deloitte Access Economics August 2015. Available from: https://docs.wixstatic.com/ugd/bfe057 b766845ec05b4256bbf5b290baa12 139.pd Accessed September 10, 2017. 
28. Martinez-Martin P, Arroyo S, Rojo-Abuin JM, et al. Burden, perceived health status, and mood among caregivers of Parkinson's disease patients. Movement Disorders. 2008;23(12):1673-1680.

29. Carter JH, Lyons KS, Lindauer A, Malcom J. Pre-death grief in Parkinson's caregivers: A pilot survey-based study. Parkinsonism \& Related Disorders. 2012;18 Suppl 3:S15-18.

30. Beach SR, Schulz R. Family caregiver factors associated with unmet needs for care of older adults. Journal of the American Geriatrics Society. 2016.

31. Ducharme F, Beaudet L, Legault A, Kergoat MJ, Levesque L, Caron C. Development of an intervention program for Alzheimer's family caregivers following diagnostic disclosure. Clinical Nursing Tesearch. 2009;18(1):4467.

32. Martire LM, Schulz R, Helgeson VS, Small BJ, Saghafi EM. Review and meta-analysis of couple-oriented interventions for chronic illness. Annals of Behavioral Medicine. 2010;40(3):325-342.

33. Kalia LV, Lang AE. Parkinson's disease. Lancet. 2015;386(9996):896-912.

34. Hoehn MM, Yahr MD. Parkinsonism: Onset, progression and mortality. Neurology. 1967;17(5):427-442.

35. Fahn S, Elton RL. Members of the UPDRS Development Committee. In: S F, CD M, DB C, M G, eds. Recent Developments in Parkinson's Disease, Vol 2. Vol 2. Florham Park, NJ Macmillan Health Care Information; 1987:153-163, 293-304.

36. Titova N, Padmakumar C, Lewis SJG, Chaudhuri KR. Parkinson's: a syndrome rather than a disease? J Neural Transm. 2017;124(8):907-914.

37. Santos-Garcia D, Mir P, Cubo E, et al. COPPADIS-2015 (COhort of Patients with PArkinson's DIsease in Spain, 2015), a global--clinical 
evaluations, serum biomarkers, genetic studies and neuroimaging-prospective, multicenter, non-interventional, long-term study on Parkinson's disease progression. BMC Neurology. 2016;16:26.

38. LoBuono DL, Taetzsch AG, Lofgren IE, Xu F, Delmonico MJ, Mahler L. Cognitive status and cardio-metabolic risk of patients with acquired brain injury and Parkinson's disease. Disability and Health Journal. 2015;9(1):134-139.

39. Kim SD, Allen NE, Canning CG, Fung VS. Postural instability in patients with Parkinson's disease. Epidemiology, pathophysiology and management. CNS Drugs. 2013;27(2):97-112.

40. Pickering RM, Grimbergen YA, Rigney U, et al. A meta-analysis of six prospective studies of falling in Parkinson's disease. Movement Disorders. 2007;22(13):1892-1900.

41. LoBuono DL, Paulin C, Furong X, Mahler L, Delmonico MJ, Lofgren IE. Parkinson's awareness and the role of a health care team in managing Parkinson's disease The Digest. 2018;53(2):13-20.

42. Cavanaugh JT, Ellis TD, Earhart GM, Ford MP, Foreman KB, Dibble LE. Toward Understanding Ambulatory Activity Decline in Parkinson Disease. Physical Therapy. 2015;95(8):1142-1150.

43. Amara AW, Chahine L, Seedorff N, et al. Self-reported physical activity levels and clinical progression in early Parkinson's disease. Parkinsonism \& Related Disorders. 2019;61:118-125.

44. Weintraub D, Comella CL, Horn S. Parkinson's disease--Part 1: Pathophysiology, symptoms, burden, diagnosis, and assessment. Am J Manag Care. 2008;14(2 Suppl):S40-48.

45. Macht M, Kaussner $\mathrm{Y}$, Moller JC, et al. Predictors of freezing in Parkinson's disease: a survey of 6,620 patients. Movement Disorders. 2007;22(7):953956. 
46. Canning CG, Paul SS, Nieuwboer A. Prevention of falls in Parkinson's disease: a review of fall risk factors and the role of physical interventions. Neurodegener Dis Manag. 2014;4(3):203-221.

47. Lajoie Y, Gallagher SP. Predicting falls within the elderly community: comparison of postural sway, reaction time, the Berg balance scale and the Activities-specific Balance Confidence (ABC) scale for comparing fallers and non-fallers. Archives of Gerontology and Geriatrics. 2004;38(1):11-26.

48. Cole MH, Silburn PA, Wood JM, Worringham CJ, Kerr GK. Falls in Parkinson's disease: kinematic evidence for impaired head and trunk control. Movement Disorders. 2010;25(14):2369-2378.

49. World Health Organization. What are neurological disorders? Online Q\&A. 2016; http://www.who.int/features/qa/55/en/. Accessed Nov 20, 2016.

50. Nonnekes J, Snijders AH, Nutt JG, Deuschl G, Giladi N, Bloem BR. Freezing of gait: a practical approach to management. Lancet Neurology. 2015;14(7):768-778.

51. Lewis GN, Byblow WD, Walt SE. Stride length regulation in Parkinson's disease: the use of extrinsic, visual cues. Brain. 2000;123 ( Pt 10):20772090.

52. Colcher A, Simuni T. Clinical manifestations of Parkinson's disease. Med Clin North Am. 1999;83(2):327-347.

53. Cavanaugh JT, Ellis TD, Earhart GM, Ford MP, Foreman KB, Dibble LE. Capturing Ambulatory activity Decline in Parkinson's disease $J$ Neurol Phys Ther. 2912;36(2):51-57.

54. Dorsey ER, Vlaanderen FP, Engelen LJ, et al. Moving Parkinson care to the home. Movement Disorders. 2016;31(9):1258-1262.

55. States RA, Sweeny TL, Rossi A, Spierer DK, Salem Y. Physical Functioning After 1, 3, and 5 Years of Exercise Among People With 
Parkinson's Disease: A Longitudinal Observational Study. J Geriatr Phys Ther. 2017;40(3):127-134.

56. Polenick CA, DePasquale N. Predictors of Secondary Role Strains Among Spousal Caregivers of Older Adults With Functional Disability. The Gerontologist. 2019;59(3):486-498.

57. Shulman LM, Gruber-Baldini AL, Anderson KE, et al. The evolution of disability in Parkinson disease. Movement disorders. 2008;23(6):790-796.

58. van Uem JMT, Cerff B, Kampmeyer M, et al. The association between objectively measured physical activity, depression, cognition, and healthrelated quality of life in Parkinson's disease. Parkinsonism \& Related Disorders. 2018;48:74-81.

59. Stegemoller EL, Nocera J, Malaty I, et al. Timed up and go, cognitive, and quality-of-life correlates in Parkinson's disease. Archives of Physical Medicine and Rehabilitation. 2014;95(4):649-655.

60. Pfeiffer RF. Gastrointestinal dysfunction in Parkinson's disease. Lancet neurology. 2003;2(2):107-116.

61. Barichella M, Cereda E, Pezzoli G. Major nutritional issues in the management of Parkinson's disease. Movement Disorders. 2009;24(13):1881-1892.

62. Andrade PA, Santos CAD, Firmino HH, Rosa COB. The importance of dysphagia screening and nutritional assessment in hospitalized patients. Einstein (Sao Paulo). 2018;16(2):eAO4189.

63. Cook IJ, Kahrilas PJ. AGA technical review on management of oropharyngeal dysphagia. Gastroenterology. 1999;116(2):455-478.

64. Kikuchi A, Baba T, Hasegawa T, et al. Hypometabolism in the supplementary and anterior cingulate cortices is related to dysphagia in Parkinson's disease: a cross-sectional and 3-year longitudinal cohort study. BMJ open. 2013;3(3). 
65. Sapir S, Ramig L, Fox C. Speech and swallowing disorders in Parkinson disease. Curr Opin Otolaryngol Head Neck Surg. 2008;16(3):205-210.

66. Baijens LW, Clave P, Cras P, et al. European Society for Swallowing Disorders - European Union Geriatric Medicine Society white paper: oropharyngeal dysphagia as a geriatric syndrome. Clinical Interventions in Aging. 2016;11:1403-1428.

67. Ekberg O, Hamdy S, Woisard V, Wuttge-Hannig A, Ortega P. Social and psychological burden of dysphagia: its impact on diagnosis and treatment. Dysphagia. 2002;17(2):139-146.

68. Miller N, Allcock L, Hildreth AJ, Jones D, Noble E, Burn DJ. Swallowing problems in Parkinson disease: frequency and clinical correlates. J Neurol Neurosurg Psychiatry. 2009;80(9):1047-1049.

69. Sura L, Madhavan A, Carnaby G, Crary MA. Dysphagia in the elderly: management and nutritional considerations. Clinical Interventions in Aging. 2012;7:287-298.

70. Kim SR, Chung SJ, Yoo SH. Factors contributing to malnutrition in patients with Parkinson's disease. International Journal of Nursing Practice. 2014;22(2):129-137.

71. Chadwick DD, Jolliffe J, Goldbart J. Adherence to eating and drinking guidelines for adults with intellectual disabilities and dysphagia. Am J Ment Retard. 2003;108(3):202-211.

72. Matsushima A, Matsushima J, Matsumoto A, et al. Analysis of resources assisting in coping with swallowing difficulties for patients with Parkinson's disease: a cross-sectional study. BMC health services research. 2016;16:276.

73. Shulman LM, Taback RL, Bean J, Weiner WJ. Comorbidity of the nonmotor symptoms of Parkinson's disease. Movement disorders : official journal of the Movement Disorder Society. 2001;16(3):507-510. 
74. Storch A, Schneider CB, Wolz M, et al. Nonmotor fluctuations in Parkinson disease: severity and correlation with motor complications. Neurology. 2013;80(9):800-809.

75. Boersma I, Jones J, Carter J, et al. Parkinson disease patients' perspectives on palliative care needs: What are they telling us? Neurology Clinical practice. 2016;6(3):209-219.

76. Gallagher DA, Lees AJ, Schrag A. What are the most important nonmotor symptoms in patients with Parkinson's disease and are we missing them? Movement Disorders. 2010;25(15):2493-2500.

77. Aarsland D, Larsen JP, Tandberg E, Laake K. Predictors of nursing home placement in Parkinson's disease: a population-based, prospective study. Journal of the American Geriatrics Society. 2000;48(8):938-942.

78. Duncan GW, Khoo TK, Yarnall AJ, et al. Health-related quality of life in early Parkinson's disease: The impact of nonmotor symptoms. Movement disorders : official journal of the Movement Disorder Society. 2013.

79. Emre M. Dementia associated with Parkinson's disease. Lancet neurology. 2003;2(4):229-237.

80. Riedel O, Schneider C, Klotsche J, Reichmann H, Storch A, Wittchen HU. [The prevalence of Parkinson's disease, associated dementia, and depression in Dresden]. Fortschr Neurol Psychiatr. 2013;81(2):81-87.

81. Nagayama H, Maeda T, Uchiyama T, et al. Anhedonia and its correlation with clinical aspects in Parkinson's disease. Journal of the neurological sciences. 2017;372:403-407.

82. Cumming RG, Le Couteur DG. Benzodiazepines and risk of hip fractures in older people: a review of the evidence. CNS drugs. 2003;17(11):825-837. 
83. Takahashi M, Tabu H, Ozaki A, Hamano T, Takeshima T, group Rs. Antidepressants for Depression, Apathy, and Gait Instability in Parkinson's Disease: A Multicenter Randomized Study. Intern Med. 2019;58(3):361368.

84. Maffoni M, Giardini A, Pierobon A, Ferrazzoli D, Frazzitta G. Stigma Experienced by Parkinson's Disease Patients: A Descriptive Review of Qualitative Studies. Parkinson's disease. 2017;2017:7203259.

85. Kudlicka A, Clare L, Hindle JV. Executive functions in Parkinson's disease: systematic review and meta-analysis. Movement disorders : official journal of the Movement Disorder Society. 2011;26(13):2305-2315.

86. Kudlicka A, Clare L, Hindle JV. Pattern of executive impairment in mild to moderate Parkinson's disease. Dementia and geriatric cognitive disorders. 2013;36(1-2):50-66.

87. Lawson RA, Yarnall AJ, Johnston F, et al. Cognitive impairment in Parkinson's disease: impact on quality of life of carers. International journal of geriatric psychiatry. 2016.

88. Raein KL, Ortiz-Hernandez S, Benge JF. Cognitive Problems in Parkinson Disease: Perspectives and Priorities of Patients and Care Partners. Cogn Behav Neurol. 2019;32(1):16-24.

89. Vossius C, Larsen JP, Janvin C, Aarsland D. The economic impact of cognitive impairment in Parkinson's disease. Movement disorders : official journal of the Movement Disorder Society. 2011;26(8):1541-1544.

90. Arie L, Herman T, Shema-Shiratzky S, Giladi N, Hausdorff JM. Do cognition and other non-motor symptoms decline similarly among patients with Parkinson's disease motor subtypes? Findings from a 5-year prospective study. Journal of Neurology. 2017;264(10):2149-2157.

91. Nagano-Saito A, Habak C, Mejia-Constain B, et al. Effect of mild cognitive impairment on the patterns of neural activity in early Parkinson's disease. Neurobiol Aging. 2014;35(1):223-231. 
92. Taylor JP, Rowan EN, Lett D, O'Brien JT, McKeith IG, Burn DJ. Poor attentional function predicts cognitive decline in patients with nondemented Parkinson's disease independent of motor phenotype. J Neurol Neurosurg Psychiatry. 2008;79(12):1318-1323.

93. Lucas $\mathrm{M}$, Chaves $\mathrm{F}$, Teixeira $\mathrm{S}$, et al. Time perception impairs sensorymotor integration in Parkinson's disease. Int Arch Med. 2013;6(1):39.

94. Kwan LC, Whitehill TL. Perception of speech by individuals with Parkinson's disease: a review. Parkinson's disease. 2011;2011:389767.

95. Chong RK, Horak FB, Woollacott MH. Parkinson's disease impairs the ability to change set quickly. Journal of the Neurological Sciences. 2000;175(1):57-70.

96. Conte A, Khan N, Defazio G, Rothwell JC, Berardelli A. Pathophysiology of somatosensory abnormalities in Parkinson disease. Nat Rev Neurol. 2013;9(12):687-697.

97. Kalf JG, de Swart BJ, Bloem BR, Munneke M. Prevalence of oropharyngeal dysphagia in Parkinson's disease: a meta-analysis. Parkinsonism \& related disorders. 2012;18(4):311-315.

98. Emek-Savas DD, Ozmus G, Guntekin B, et al. Decrease of Delta Oscillatory Responses in Cognitively Normal Parkinson's Disease. Clin EEG Neurosci. 2017;48(5):355-364.

99. Palomar FJ, Diaz-Corrales F, Carrillo F, Fernandez-del-Olmo M, Koch G, Mir P. Sensory perception changes induced by transcranial magnetic stimulation over the primary somatosensory cortex in Parkinson's disease. Movement Disorders.. 2011;26(11):2058-2064.

100. Shanahan J, Bhriain ON, Morris ME, Volpe D, Clifford AM. Irish set dancing 
classes for people with Parkinson's disease: The needs of participants and dance teachers. Complement Ther Med. 2016;27:12-17

101. Fox CM, Ramig LO. Vocal sound pressure level and self-perception of speech

and voice in men and women with idiopathic Parkinson disease.

American Journal of Speech Language Pathology. 1997;6(2):85-94.

102. Breitenstein C, van Lancker D, Kempler D, Daum I, Waters CH. The contributors of working memory to the perception of emotional prosody in

Parkinson's disease. Brain and Llanguage. 1998;56:241-251.

103. Rascol O, Brooks DJ, Korczyn AD, De Deyn PP, Clarke CE, Lang AE. A five

year study of the incidence of dyskinesia in patients with early Parkinson's disease who were treated with ropinirole or levodopa. The New England Journal of Medicine. 2000;342(20):1484-1491.

104. Borovac JA. Side effects of a dopamine agonist therapy for Parkinson's disease: a mini-review of clinical pharmacology. Yale $\mathrm{J}$ Biol Med. 2016;89(1):37-47.

105. Stowe RL, Ives NJ, Clarke C, et al. Dopamine agonist therapy in early Parkinson's disease. Cochrane Database Syst Rev. 2008(2):CD006564.

106. Obeso JA, Rodriguez-Oroz MC, Chana P, Lera G, Rodriguez M, Olanow CW.

The evolution and origin of motor complications in Parkinson's disease. Neurology. 2000;55(11 Suppl 4):S13-20; discussion S21-13.

107. Gould E, Mitty E. Medication adherence is a partnership, medication compliance is not. Geriatr Nurs. 2010;31(4):290-298.

108. Shin JY, Habermann B, Pretzer-Aboff I. Challenges and strategies of medication adherence in Parkinson's disease: A qualitative study. Geriatr Nurs. 2015;36(3):192-196. 
109. Wei YJ, Palumbo FB, Simoni-Wastila L, et al. Antiparkinson drug adherence

and its association with health care utilization and economic outcomes in a Medicare Part D population. Value Health. 2014;17(2):196-204.

110. Tarrants ML, Denarie MF, Castelli-Haley J, Millard J, Zhang D. Drug therapies

for Parkinson's disease: A database analysis of patient compliance and persistence. Am J Geriatr Pharmacother. 2010;8(4):374-383.

111. Richy FF, Pietri G, Moran KA, Senior E, Makaroff LE. Compliance with pharmacotherapy and direct healthcare costs in patients with Parkinson's disease: a retrospective claims database analysis. Appl Health Econ Health Policy. 2013;11(4):395-406.

112. Liao CY, Wu MF, Poon SK, et al. Improving medication safety by cloud technology: Progression and value-added applications in Taiwan. International Journal of Medical Informatics. 2019;126:65-71.

113. Mansouri A, Taslimi S, Badhiwala JH, et al. Deep brain stimulation for Parkinson's disease: meta-analysis of results of randomized trials at varying lengths of follow-up. Journal of Neurosurgery. 2018;128(4):1199-1213.

114. Weaver FM, Stroupe KT, Smith B, et al. Survival in patients with Parkinson's disease after deep brain stimulation or medical management. Movement disorders : official journal of the Movement Disorder Society. 2017;32(12):1756-1763.

115. Stroupe KT, Smith B, Weaver FM, et al. Healthcare Utilization and Costs for

Patients With Parkinson's Disease After Deep Brain Stimulation. Mov Disord Clin Pract. 2019;6(5):369-378. 
116. Farley BG, Koshland GF. Training BIG to move faster: the application of the speed-amplitude relation as a rehabilitation strategy for people with Parkinson's disease. Exp Brain Res. 2005;167(3):462-467.

117. Fox C, Ebersbach G, Ramig L, Sapir S. LSVT LOUD and LSVT BIG: Behavioral Treatment Programs for Speech and Body Movement in Parkinson Disease. Parkinson's disease. 2012;2012:391946.

118. Dashtipour K, Johnson E, Kani C, et al. Effect of exercise on motor and nonmotor symptoms of Parkinson's disease. Parkinson's disease. 2015;2015:586378.

119. Sumec R, Filip P, Sheardova K, Bares M. Psychological Benefits of Nonpharmacological Methods Aimed for Improving Balance in Parkinson's Disease: A Systematic Review. Behavioural neurology. 2015;2015:620674.

120. Shulman LM, Katzel LI, Ivey FM, et al. Randomized clinical trial of 3 types of physical exercise for patients with Parkinson disease. JAMA Neurology. 2013;70(2):183-190.

121. Corcos DM, Robichaud JA, David FJ, et al. A two-year randomized controlled trial of progressive resistance exercise for Parkinson's disease. Movement Disorders. 2013;28(9):1230-1240.

122. Li F, Harmer $\mathrm{P}$, Fitzgerald $\mathrm{K}$, et al. Tai chi and postural stability in patients with Parkinson's disease. The New England Journal of Medicine. 2012;366(6):511-519.

123. Duncan RP, Earhart GM. Randomized controlled trial of communitybased dancing to modify disease progression in Parkinson disease. Neurorehabilitation and Neural Repair. 2012;26(2):132-143.

124. Alberts JL, Linder SM, Penko AL, Lowe MJ, Phillips M. It is not about the bike, it is about the pedaling: forced exercise and Parkinson's disease. Exerc Sport Sci Rev. 2011;39(4):177-186. 
125. Combs SA, Diehl MD, Chrzastowski C, et al. Community-based group exercise for persons with Parkinson disease: a randomized controlled trial. NeuroRehabilitation. 2013;32(1):117-124.

126. Caballol N, Marti MJ, Tolosa E. Cognitive dysfunction and dementia in Parkinson disease. Movement Disorders. 2007;22 Suppl 17:S358-366.

127. Argolo N, Sampaio M, Pinho P, Melo A, Nobrega AC. Do swallowing exercises improve swallowing dynamic and quality of life in Parkinson's disease? NeuroRehabilitation. 2013;32(4):949-955.

128. Sapir S, Ramig LO, Fox CM. Intensive voice treatment in Parkinson's disease: Lee Silverman Voice Treatment. Expert Review of Neurotherapeutics. 2011;11(6):815-830.

129. Mahler LA, Ramig LO, Fox C. Evidence-based treatment of voice and speech disorders in Parkinson disease. Curr Opin Otolaryngol Head Neck Surg. 2015;23(3):209-215.

130. Wolrd Health Organization. Towards a Common Language for Functioning, Disability and Health: ICF- The International Classification of Functioning, Disabi;ity and Health Geneva, Switzerland 2002. Available from: https://www.who.int/classifications/icf/icfbeginnersguide.pdf Accessed July 30,2019

131. Vojciechowski AS, Zotz TGG, Loureiro APC, Israel VL. The International Classification of Functioning, Disability, and Health as Applied to Parkinson's disease: A Literature Review Advances in Parkinson's Disease. 2016;5:29-40.

132. Wild LB, de Lima DB, Balardin JB, et al. Characterization of cognitive and motor performance during dual-tasking in healthy older adults and patients with Parkinson's disease. Journal of Neurology. 2013;260:580589.

133. Kudlicka A, Clare L, Hindle JV. Quality of life, health status and caregiver burden in Parkinson's disease: relationship to executive functioning. International Journal of Geriatric Psychiatry. 2013. 
134. Kelly VE, Eusterbrock AJ, Shumway-Cook A. A review of dual-task walking deficits in people with Parkinson's disease: motor and cognitive contributions, mechanisms, and clinical implications. Parkinson's disease. 2012;2012:918719.

135. Roth DL, Fredman L, Haley WE. Informal caregiving and its impact on health: a reappraisal from population-based studies. The Gerontologist. 2015;55(2):309-319.

136. Prizer LP, Browner N. The integrative care of Parkinson's disease: a systematic review. Journal of Parkinson's disease. 2012;2(2):79-86.

137. Kessler D, Hauteclocque J, Grimes D, Mestre T, Coted D, Liddy C. Development of the Integrated Parkinson's Care Network (IPCN): using co-design to plan collaborative care for people with Parkinson's disease. Quality of Life Research. 2019;28(5):1355-1364.

138. Willis AW, Schootman M, Evanoff BA, Perlmutter JS, Racette BA. Neurologist care in Parkinson disease: a utilization, outcomes, and survival study. Neurology. 2011;77(9):851-857.

139. Achey M, Aldred JL, Aljehani N, et al. The past, present, and future of telemedicine for Parkinson's disease. Movement disorders : official journal of the Movement Disorder Society. 2014;29(7):871-883.

140. Ball N, Teo WP, Chandra S, Chapman J. Parkinson's Disease and the Environment. Front Neurol. 2019;10:218.

141. Duvoisin RC, Sage J. Chapter 5: Principles of treatment. In: Parkinson's disease: A guide for patient and family. Lippincott Williams \& Wilkins; 2001:49.

142. Constantinescu G, Theodoros D, Russell T, Ward E, Wilson S, Wootton $\mathrm{R}$. Treating disordered speech and voice in Parkinson's disease online: 
A randomized controlled non-inferiority trial. International Journal of Language \& Communication Disorders. 2011;46(1):1-16.

143. Monticone M, Ambrosini E, Laurini A, Rocca B, Foti C. In-patient multidisciplinary rehabilitation for Parkinson's disease: A randomized controlled trial. Movement Disorders. 2015;30(8):1050-1058.

144. Vikdahl M, Domellof ME, Forsgren L, Haglin L. Olfactory function, eating ability, and visceral obesity associated with MMSE three years after Parkinson's disease diagnosis. The Journal of Nutrition, Health \& Aging. 2015;19(9):894-900.

145. Cherry C, Macredie RD. The importance of context in information system design: An assessment of participatory design. Requirements Engineering. 1999;4(2):103-114.

146. van Velsen L, Illario M, Jansen-Kosterink S, et al. A community-based, technology-supported health service for detecting and preventing frailty among older adults: A participatory design development process. Journal of Aging Research. 2015;2015:216084.

147. Swallow D, Petrie H, Power C, Lewis A, Edwards AD. Involving older adults in the technology design process: A case study on mobility and wellbeing in the built environment. Studies in Health Technology and Informatics. 2016;229:615-623.

148. Beaudet L, Ducharme F, L'Ecuyer N, Chouinard S, Jodoin N, Panisset M. Development and evaluation of a dyadic intervention for elderly couples living with moderate-stage Parkinson disease. Applied Nursing Research. 2015;28(4):e21-27.

149. Davis FD, Bagozzi RP, Warshaw PR. User acceptance of computer technology: A comparison of two theoretical models. Manage Sci. 1989;35(8):982-1003.

150. Chen K, Chan AH. Gerontechnology acceptance by elderly Hong Kong Chinese: a senior technology acceptance model (STAM). Ergonomics. 2014;57(5):635-652. 
151. Digital Health. Medical Devices. 2016 Available from: https://www.fda.gov/MedicalDevices/DigitalHealth/default.htm. Accessed June 30, 2017.

152. Fox S. Online Health Search 2006. Washington, DC PEW Research Center;2006

153. Fox S. Medicine 2.0: Peer-to-peer healthcare. Pew Research Center;2011

154. Pew Research Center: Internet \& Technology. Mobile Fact Sheet. 2018 Available from: www.pewinternet.org/fact-sheet/mobile/ Accessed July 122019

155. Pasluosta CF, Gassner H, Winkler J, Klucken J, Eskofier BM. An emerging era in the management of Parkinson's disease: Wearable technologies and the internet of things. IEEE Journal of Biomedical and Health Informatics. 2015;19(6):1873-1881.

156. Dorsey ER, Venkataraman V, Grana MJ, et al. Randomized controlled clinical trial of "virtual house calls" for Parkinson disease. JAMA neurology. 2013;70(5):565-570.

157. Bloem BR, de Vries NM, Ebersbach G. Nonpharmacological treatments for patients with Parkinson's disease. Movement Disorders. 2015.

158. van der Marck MA, Bloem BR, Borm GF, Overeem S, Munneke M, Guttman M. Effectiveness of multidisciplinary care for Parkinson's disease: a randomized, controlled trial. Movement Disorders. 2013;28(5):605-611.

159. Singh R, Pentland B, Hunter J, Provan F. Parkinson's disease and driving ability. J Neurol Neurosurg Psychiatry. 2007;78(4):363-366. 
160. Lakshminarayana $R$, Wang $D$, Burn $D$, et al. Using a smartphone-based self-management platform to support medication adherence and clinical consultation in Parkinson's disease. NPJ Parkinsons Dis. 2017;3:2.

161. Lakshminarayana R, Wang D, Burn D, et al. Smartphone- and internetassisted self-management and adherence tools to manage Parkinson's disease (SMART-PD): Study protocol for a randomised controlled trial (v7; 15 August 2014). Trials. 2014;15:374.

162. Healthcare Information and Management Systems Society. The Department of Veterans Affairs \#mHealth case study. 2014; http://www.himss.org/department-veterans-affairs-mhealth-case-study. Accessed December 30, 2016.

163. Wilkinson JR, Spindler M, Wood SM, et al. High patient satisfaction with telehealth in Parkinson disease: A randomized controlled study. Neurology Clinical practice. 2016;6(3):241-251.

164. Dorsey ER, Achey MA, Beck CA, et al. National randomized controlled trial of virtual house calls for people with Parkinson's disease: Interest and barriers. Telemedicine Journal and e-health. 2016;22(7):590-598.

165. Schulz R, Beach SR, Matthews JT, Courtney K, De Vito Dabbs A, Mecca LP. Caregivers' willingness to pay for technologies to support caregiving. The Gerontologist. 2016;56(5):817-829.

166. Espay AJ, Hausdorff JM, Sanchez-Ferro A, et al. A roadmap for implementation of patient-centered digital outcome measures in Parkinson's disease obtained using mobile health technologies. Movement disorders. 2019;34(5):657-663.

167. Qiang JK, Marras C. Telemedicine in Parkinson's disease: A patient perspective at a tertiary care centre. Parkinsonism \& Related Disorders. 2015;21(5):525-528.

168. Duroseau N, Abramson T, Pergament K, et al. Acceptance of technology-based tools in a sample of Parkinson's patients. Chronic IIIn. 2017;13(1):3-13. 
169. Tindall LR, Huebner RA. The impact of an application of telerehabilitation technology on caregiver burden. Int J Telerehabil. 2009;1(1):3-8.

170. Klaassen B, van Beijnum BJ, Hermens HJ. Usability in telemedicine systems: A literature survey. International Journal of Medical Informatics. 2016;93:57-69.

171. Ferreira JJ, Godinho C, Santos AT, et al. Quantitative home-based assessment of Parkinson's symptoms: the SENSE-PARK feasibility and usability study. BMC neurology. 2015;15:89.

172. Visser LM, Bleijenbergh IL, Benschop YW, Van Riel AC, Bloem BR. Do online communities change power processes in healthcare? Using case studies to examine the use of online health communities by patients with Parkinson's disease. BMJ open. 2016;6(11):e012110.

173. Stillerova T, Liddle J, Gustafsson L, Lamont R, Silburn P. Could everyday technology improve access to assessments? A pilot study on the feasibility of screening cognition in people with Parkinson's disease using the Montreal Cognitive Assessment via internet videoconferencing. Australian Occupational Therapy Journal. 2016;63(6):373-380.

174. Stamford JA, Schmidt PN, Friedl KE. What engineering technology could do for quality of life in Parkinson's disease: A review of current needs and opportunities. IEEE Journal of Biomedical and Health Informatics. 2015;19(6):1862-1872.

175. Banbury A, Chamberlain D, Nancarrow S, Dart J, Gray L, Parkinson L. Can videoconferencing affect older people's engagement and perception of their social support in long-term conditions management: A social network analysis from the telehealth literacy project. Health Soc Care Community. 2016;25(3):938-950. 
176. Shah SP, Glenn GL, Hummel EM, et al. Caregiver tele-support group for Parkinson's disease: A pilot study. Geriatr Nurs. 2015;36(3):207211.

177. Kreps GL, Neuhauser L. New directions in eHealth communication: opportunities and challenges. Patient Education and Counseling. 2010;78(3):329-336.

178. Eysenbach G. What is e-health? Journal of Medical Internet Research. 2001;3(2):E20.

179. Milligan C, Roberts C, Mort M. Telecare and older people: Who cares where? Social Science \& Medicine. 2011;72(3):347-354.

180. Atkin $P$, Barrett $D$. Benefits of telemonitoring in the care of patients with heart failure. Nursing Standard. 2012;27(4):44-48.

181. Segar J, Rogers A, Salisbury C, Thomas C. Roles and identities in transition: Boundaries of work and inter-professional relationships at the interface between telehealth and primary care. Health Soc Care Community. 2013;21(6):606-613.

182. Solberg LB. The benefits of online health communities. Virtual Mentor. 2014;16(4):270-274.

183. Hoffmann T, Russell T, Thompson L, Vincent A, Nelson M. Using the Internet to assess activities of daily living and hand function in people with Parkinson's disease. NeuroRehabilitation. 2008;23(3):253-261.

184. Sekimoto S, Oyama G, Hatano T, et al. A Randomized Crossover Pilot Study of Telemedicine Delivered via iPads in Parkinson's Disease. Parkinson's disease. 2019;2019:9403295.

185. Agency for Healthcare Research and Quality.Telehealth: Mapping the evidence for patient outcomes from systematic review . 2016; https://effectivehealthcare.ahrq.gov/ehc/products/624/2254/telehealthreport-160630.pdf. Accessed December 22, 2016. 
186. Monje MHG, Foffani G, Obeso J, Sanchez-Ferro A. New Sensor and Wearable Technologies to Aid in the Diagnosis and Treatment Monitoring of Parkinson's Disease. Annu Rev Biomed Eng. 2019;21:111-143.

187. Ozanne A, Johansson D, Hallgren Graneheim U, Malmgren K, Bergquist F, Alt Murphy M. Wearables in epilepsy and Parkinson's disease-A focus group study. Acta neurologica Scandinavica. 2018;137(2):188-194.

188. Colon-Semenza C, Latham NK, Quintiliani LM, Ellis TD. Peer Coaching Through mHealth Targeting Physical Activity in People With Parkinson Disease: Feasibility Study. JMIR Mhealth Uhealth. 2018;6(2):e42.

189. Arora S, Venkataraman $\mathrm{V}$, Zhan $\mathrm{A}$, et al. Detecting and monitoring the symptoms of Parkinson's disease using smartphones: A pilot study. Parkinsonism \& Related Disorders. 2015;21(6):650-653.

190. Patel S, Chen BR, Buckley T, et al. Home monitoring of patients with Parkinson's disease via wearable technology and a web-based application. Conf Proc IEEE Eng Med Biol Soc. 2010;2010:4411-4414.

191. Attard A, Coulson NS. A thematic analysis of patient communication in Parkinson's disease online support group discussion forums. Computers in Human Behavior. 2012;38:500-506.

192. Fox S, Purcell K. Chronic disease and the interent Pew Research Center; 2010 Available from: https://www.pewinternet.org/2010/03/24/chronic-disease-and-theinternet/ Accessed Dceember 12, 2018

193. Pew Research Center. Social Media Fact Sheet. June 12, 2019, Available from: https://www.pewinternet.org/fact-sheet/social-media/ Accesed August 10, 2019

194. Cummings JN, Sproull L, Kiesler SB. Beyond hearing: where real-world and online support meet. Group Dyanamics: Theory, Research, and Practice. 2002;6(78-88). 
195. Shapira AL, Handzel R, Korczyn AD. The Lived Experience of Parkinson's Disease: A Content Analysis of Parkinson's Patients' Blogs. Isr Med Assoc J. 2017;19(11):685-690.

196. Winett RA, Anderson ES, Wojcik JR, Winett SG, Moore S, Blake C. Guide to health: A randomized controlled trial of the effects of a completely web-based intervention on physical activity, fruit and vegetable consumption, and body weight. Translational Behavioral Medicine. 2011;1(1):165-174.

197. Alexander GL, McClure JB, Calvi JH, et al. A randomized clinical trial evaluating online interventions to improve fruit and vegetable consumption. American Journal of Public Health. 2010;100(2):319-326.

198. Olson CM. Behavioral nutrition interventions using e- and $m$-Health communication technologies: A narrative review. Annual Review of Nutrition. 2016;36:647-664.

199. Springvloet L, Lechner L, de Vries H, Candel MJ, Oenema A. Shortand medium-term efficacy of a web-based computer-tailored nutrition education intervention for adults including cognitive and environmental feedback: Randomized controlled trial. Journal of medical Internet research. 2015;17(1):e23.

200. Martin CK, Han H, Coulon SM, Allen HR, Champagne CM, Anton SD. A novel method to remotely measure food intake of free-living individuals in real time: the remote food photography method. The British journal of nutrition. 2009;101(3):446-456.

201. Ben Neriah D, Geliebter A. Weight Loss Following Use of a Smartphone Food Photo Feature: Retrospective Cohort Study. JMIR Mhealth Uhealth. 2019;7(6):e11917.

202. Academy of Nutrition and Dietetics: Definitions of terms list; eat right. Chicago, IL, USA Academy of Nutrition and Dietetics 2017 
203. Ventura Marra M, Lilly CL, Nelson KR, Woofter DR, Malone J. A Pilot Randomized Controlled Trial of a Telenutrition Weight Loss Intervention in Middle-Aged and Older Men with Multiple Risk Factors for Cardiovascular Disease. Nutrients. 2019;11(2).

204. Chi NC, Demiris G. A systematic review of telehealth tools and interventions to support family caregivers. J Telemed Telecare. 2015;21(1):37-44.

205. Fox S. Strategic learning for health care in 2010. Pew Internet and American Life Project 2010.

206. Gibson MJ, House A. Valuing the invaluable: a new look at the economic value of family caregiving. Issue Brief. Washington, D.C. : Public Policy Instiution, American Association of Retired Persons; 2007.

207. Macan Yadrich D, Fitzgerald SA, Werkowitch M, Smith CE. Creating patient and family education web sites: Assuring accessibility and usability standards Comput Inform Nurs. 2012;30(1):45-54.

208. Marziali E, Donahue P, Crossin G. Caring for others: Internet health care support intervention for family caregivers of persons with Alzehimer's, stroke, or Parkinson's disease. Families in Society: The Journal of Contemproary Social Services 2005;86(3):375-383.

209. Frisancho AR. New standards of weight and body composition by frame size and height for assessment of nutritional status of adults and the elderly. Am J Clin Nutr. 1984;40(4):808-819.

210. Malnutrition Advisory Group. Guidelines for detection and management of malnutrition. 2000. Accessed May 2, 2015.

211. Carmo TPS, Ferraz AC. Nutritional assessment and the use of levodopa with protein meals among patients with Parkinson's disease in the city of Macaé, Rio de Janeiro, Brazil. Conference Proceedings. 2016. 
212. Barichella M, Cereda E, Madio C, et al. Nutritional risk and gastrointestinal dysautonomia symptoms in Parkinson's disease outpatients hospitalised on a scheduled basis. The British Journal of Nutrition. 2013;110(2):347-353.

213. Chaudhuri KR, Martinez-Martin P, Schapira AH, et al. International multicenter pilot study of the first comprehensive self-completed nonmotor symptoms questionnaire for Parkinson's disease: the NMSQuest study. Movement Sisorders. 2006;21(7):916-923.

214. Lorefalt B, Ganowiak W, Palhagen S, Toss G, Unosson M, Granerus AK. Factors of importance for weight loss in elderly patients with Parkinson's disease. Acta Neurologica Scandinavica. 2004;110(3):180187.

215. Delmonico MJ, Harris TB, Lee JS, et al. Alternative definitions of sarcopenia, lower extremity performance, and functional impairment with aging in older men and women. Journal of the American Geriatrics Society. 2007;55(5):769-774.

216. Bailey RL. Dietary screening tool identifies nutritional risk in older adults. Am J Clin Nutr. 2009;90:177-183.

217. Cassani E, Barichella M, Ferri V, et al. Dietary habits in Parkinson's disease: Adherence to Mediterranean diet. Parkinsonism \& Related Disorders. 2017;42:40-46.

218. Virmani T, Tazan S, Mazzoni P, Ford B, Greene PE. Motor fluctuations due to interaction between dietary protein and levodopa in Parkinson's disease. J Clin Mov Disord. 2016;3:8.

219. Wills AA, Perez A, Wang J, et al. Association between change in body mass index, Unified Parkinson's Disease Rating Scale Scores, and survival among persons with Parkinson disease: Secondary analysis of longitudinal data From NINDS exploratory trials in Parkinson disease long-term study 1. JAMA Neurology. 2016;73(3):321-328. 
220. Sheard JM, Ash S, Silburn PA, Kerr GK. Prevalence of malnutrition in Parkinson's disease: a systematic review. Nutrition Reviews. 2011;69(9):520-532.

221. Chen CM, Chang WC, Lan TY. Identifying factors associated with changes in physical functioning in an older population. Geriatrics \& Gerontology International. 2014.

222. Felice VD, Quigley EM, Sullivan AM, O'Keeffe GW, O'Mahony SM. Microbiota-gut-brain signalling in Parkinson's disease: Implications for non-motor symptoms. Parkinsonism \& Related Disorders. 2016;27:1-8.

223. Cereda E, Barichella M, Pezzoli G. Controlled-protein dietary regimens for Parkinson's disease. Nutritional Neuroscience. 2010;13(1):29-32.

224. Lindskov S, Sjoberg K, Hagell P, Westergren A. Weight stability in Parkinson's disease. Nutritional Neuroscience. 2015.

225. Escott-Stump S. In: Nutrition and Diagnosis - Related Care. 7th Edition ed. Baltimore, MD: Lippincott Williams \& Wilkins 2011:602-603.

226. Marczewska A, De Notaris R, Sieri S, Barichella M, Fusconi E, Pezzoli G. Protein intake in Parkinsonian patients using the EPIC food frequency questionnaire. Movement Disorders. 2006;21(8):1229-1231.

227. Barichella M, Marczewska AM, Mariani C, Landi A, Vairo A, Pezzoli G. Body weight gain rate in patients with Parkinson's disease and deep brain stimulation. Movement disorders. 2003;18(11):1337-1340.

228. Chen JJ, Marsh L. Anxiety in Parkinson's disease: identification and management. Ther Adv Neurol Disord. 2014;7(1):52-59.

229. Bachmann CG, Zapf A, Brunner E, Trenkwalder C. Dopaminergic treatment is associated with decreased body weight in patients with Parkinson's disease and dyskinesias. European Journal of Neurology. 2009;16(8):895-901. 
230. Aiello M, Eleopra R, Rumiati RI. Body weight and food intake in Parkinson's disease. A review of the association to non-motor symptoms. Appetite. 2014;84::204-211.

231. Clark BC, Manini TM. Sarcopenia $=/=$ dynapenia. The Journals of Gerontology Series A, Biological Sciences and Medical Sciences. 2008;63(8):829-834.

232. Cruz-Jentoft AJ, Landi F, Schneider SM, et al. Prevalence of and interventions for sarcopenia in ageing adults: a systematic review. Report of the International Sarcopenia Initiative (EWGSOP and IWGS). Age and Ageing. 2014;43(6):748-759.

233. Bernhardt D, Muller HP, Ludolph AC, Dupuis L, Kassubek J. Body fat distribution in Parkinson's disease: An MRI-based body fat quantification study. Parkinsonism \& Related Disorders. 2016;33:84-89.

234. Vikdahl M, Carlsson M, Linder J, Forsgren L, Haglin L. Weight gain and increased central obesity in the early phase of Parkinson's disease. Clinical Nutrition. 2014;33(6):1132-1139.

235. Barichella M, Pinelli G, lorio L, et al. Sarcopenia and dynapenia in patients with Parkinsonism. Journal of the American Medical Directors Association. 2016;17(7):640-646.

236. Cereda E, Cassani E, Barichella M, et al. Low cardiometabolic risk in Parkinson's disease is independent of nutritional status, body composition and fat distribution. Clinical Nutrition. 2012;31(5):699-704.

237. Cassani E, Cereda E, Barichella M, et al. Cardiometabolic factors and disease duration in patients with Parkinson's disease. Nutrition. 2013;29(11-12):1331-1335.

238. Wang G, Wan $Y$, Cheng $Q$, et al. Malnutrition and associated factors in Chinese patients with Parkinson's disease: Results from a pilot investigation. Parkinsonism \& Related Disorders. 2010;16(2):119-123. 
239. Chen H, Zhang SM, Hernan MA, Willett WC, Ascherio A. Weight loss in Parkinson's disease. Annals of Neurology. 2003;53(5):676-679.

240. Durrieu G, ME LL, Rascol O, Senard JM, Rascol A, Montastruc JL. Parkinson's disease and weight loss: a study with anthropometric and nutritional assessment. Clinical Autonomic Research. 1992;2(3):153157.

241. Uc EY, Struck LK, Rodnitzky RL, Zimmerman B, Dobson J, Evans WJ. Predictors of weight loss in Parkinson's disease. Movement Disorders. 2006;21(7):930-936.

242. Sheard JM, Ash S, Mellick GD, Silburn PA, Kerr GK. Malnutrition in a sample of community-dwelling people with Parkinson's disease. PloS one. 2013;8(1):e53290.

243. Chahine LM, Dos Santos C, Fullard M, et al. Modifiable vascular risk factors, white matter disease and cognition in early Parkinson's disease. European Journal of Neurology. 2019;26(2):246-e218.

244. Knopman D, Boland LL, Mosley T, et al. Cardiovascular risk factors and cognitive decline in middle-aged adults. Neurology. 2001;56(1):42-48.

245. Wei Q, Wang H, Tian Y, Xu F, Chen X, Wang K. Reduced serum levels of triglyceride, very low density lipoprotein cholesterol and apolipoprotein B in Parkinson's disease patients. PloS one. 2013;8(9):e75743.

246. Mischley LK, Lau RC, Bennett RD. Role of Diet and Nutritional Supplements in Parkinson's Disease Progression. Oxid Med Cell Longev. 2017;2017:6405278.

247. Sato Y, Kikuyama M, Oizumi K. High prevalence of vitamin D deficiency and reduced bone mass in Parkinson's disease. Neurology. 1997;49(5):1273-1278. 
248. da Silva TM, Munhoz RP, Alvarez C, et al. Depression in Parkinson's disease: a double-blind, randomized, placebo-controlled pilot study of omega-3 fatty-acid supplementation. Journal of affective disorders. 2008;111(2-3):351-359.

249. Sheard JM, Ash S. Current practice in nutrition diagnosis and intervention for the management of Parkinson's disease in Australia and Canada. Nutr Diet. 2015;72:30-35.

250. Baroni L, Bonetto C, Tessan F, et al. Pilot dietary study with normoproteic protein-redistributed plant-food diet and motor performance in patients with Parkinson's disease. Nutritional neuroscience. 2011;14(1):1-9.

251. Cereda E, Barichella M, Pedrolli C, Pezzoli G. Low-protein and proteinredistribution diets for Parkinson's disease patients with motor fluctuations: A systematic review. Movement Disorders. 2010;25(13):2021-2034.

252. Rezak M. Nutritional Issues in Parkinson's Disease. In. Vol 2017. American Parkinson's Disease Association

253. Diet and Nutrition. Parkinson's Disease Foundation. . https://www.parkinson.org/Living-with-Parkinsons/ManagingParkinsons/Diet-and-Nutrition.

254. Taghizadeh M, Tamtaji OR, Dadgostar E, et al. The effects of omega-3 fatty acids and vitamin E co-supplementation on clinical and metabolic status in patients with Parkinson's disease: A randomized, double-blind, placebo-controlled trial. Neurochem Int. 2017;108:183-189.

255. Wang L, Xiong N, Huang J, et al. Protein-Restricted Diets for Ameliorating Motor Fluctuations in Parkinson's Disease. Frontiers in Aging Neuroscience. 2017;9:206.

256. Gillespie NG, Mena I, Cotzias GC, Bell MA. Diets affecting treatment of parkinsonism with levodopa. Journal of the American Dietetic Association. 1973;62(5):525-528. 
257. Sheard JM, Ash S, Mellick GD, Silburn PA, Kerr GK. Improved nutritional status is related to improved quality of life in Parkinson's disease. BMC Neurology. 2014;14:212.

258. Fowler CN, Kott K, Wicks MN, Rutledge C. An Interprofessional Virtual Healthcare Neighborhood: Effect on Self-Efficacy and Sleep Among Caregivers of Older Adults With Dementia. Journal of Gerontological Nursing. 2016;42(11):39-47.

259. Berry RA, Murphy JF. Well-being of caregivers of spouses with Parkinson's disease. Clinical Nursing Research. 1995;4(4):373-386.

260. Bognar S, DeFaria AM, O'Dwyer C, et al. More than just dancing: experiences of people with Parkinson's disease in a therapeutic dance program. Disability and Rehabilitation. 2016:1-6.

261. Dorsey ER, Voss TS, Shprecher DR, et al. A U.S. survey of patients with Parkinson's disease: satisfaction with medical care and support groups. Movement Disorders. 2010;25(13):2128-2135.

262. Meleis Al, Sawyer LM, Im EO, Hilfinger Messias DK, Schumacher K. Experiencing transitions: An emerging middle-range theory. Adv Nurs Sci. 2000;23(1):12-28.

263. Ducharme FC, Levesque LL, Lachance LM, et al. "Learning to become a family caregiver" efficacy of an intervention program for caregivers following diagnosis of dementia in a relative. The Gerontologist. 2011;51(4):484-494.

264. Navarta-Sanchez MV, Caparros N, Fernandez MR, Diaz De Cerio Ayesa S, Ursua Sesma ME, Portillo MC. Core elements to understand and improve coping with Parkinson's disease in patients and family carers: A focus group study. Journal of Advanced Nursing. 2017.

265. Navarta-Sanchez MV, Ursua ME, Riverol Fernandez M, et al. Implementation of a multidisciplinary psychoeducational intervention for Parkinson's disease patients and carers in the community: study protocol. BMC Fam Pract. 2018;19(1):45. 
266. Schipper K, Dauwerse L, Hendrikx A, Leedekerken JW, Abma TA. Living with Parkinson's disease: priorities for research suggested by patients. Parkinsonism \& Related Disorders. 2014;20(8):862-866.

267. Greenwell K, Gray WK, van Wersch A, van Schaik P, Walker R. Predictors of the psychosocial impact of being a carer of people living with Parkinson's disease: a systematic review. Parkinsonism \& Related Disorders. 2015;21(1):1-11.

268. Hounsgaard L, Pedersen B, Wagner L. The daiy living for infornal caregivers with a partner with Parkinson's disease - an interview study of women's experiences of care decisions and self-management $J$ Nurs Health Chronic IIIn. 2011;3:504-412.

269. Peters M. Quality of life and burden in caregivers for patients with PD. Focus Parkinsons Dis. 2014;24(1):44-48.

270. Bloem BR, Stocchi F. Move for Change Part III: a European survey evaluating the impact of the EPDA Charter for People with Parkinson's Disease. European Journal of Neurology. 2015;22(1):133-141, e138139.

271. Martinez-Martin $\mathrm{P}$, Macaulay D, Jalundhwala $\mathrm{YJ}$, et al. The long-term direct and indirect economic burden among Parkinson's disease caregivers in the United States. Movement disorders : official journal of the Movement Disorder Society. 2019;34(2):236-245.

272. Dahodwala N, Shah K, He Y, et al. Sex disparities in access to caregiving in Parkinson disease. Neurology. 2018;90(1):e48-e54.

273. Aneshensel CS, Pearlin LI, Mullan JT, Zarit SH, Whitlatch CJ. Profiles in caregiving. The unexpected career. San Diego, CA: Academic Press; 1995.

274. Pinquart M, Sorensen S. Correlates of physical health of informal caregivers: a meta-analysis. J Gerontol B Psychol Sci Soc Sci. 2007;62(2):P126-137. 
275. Badr H, Herbert K, Reckson B, Rainey H, Sallam A, Gupta V. Unmet needs and relationship challenges of head and neck cancer patients and their spouses. J Psychosoc Oncol. 2016;34(4):336-346.

276. Chattillion EA, Ceglowski J, Roepke SK, et al. Pleasant events, activity restriction, and blood pressure in dementia caregivers. Health Psychol. 2013;32(7):793-801.

277. Moore RC, Harmell AL, Chattillion E, Ancoli-Israel S, Grant I, Mausbach BT. PEAR model and sleep outcomes in dementia caregivers: influence of activity restriction and pleasant events on sleep disturbances. International Psychogeriatrics. 2011;23(9):1462-1469.

278. Habermann B, Shin JY. Preferences and concerns for care needs in advanced Parkinson's disease: A qualitative study of couples. Journal of clinical nursing. 2017;26(11-12):1650-1656.

279. Smith LJ, Shaw RL. Learning to live with Parkinson's disease in the family unit: An interpretative phenomenological analysis of well-being. Med Health Care Philos. 2017;20(1):13-21.

280. Bandura A. Self-efficacy: toward a unifying theory of behavioral change. Psychological review. 1977;84(2):191-215. 


\section{APPENDIX B}

\section{EXTENDED METHODOLOGY}

\section{Overview}

The completed studies are part two major studies / projects at the University of Rhode Island. Project 1 is part of a 5-year longitudinal study examining the change in nutrition, physical function, cognition and swallowing among those living with neurological disorders and neurotypical participants. Project 2 is a formative, mixed-methods analysis, assessing people with Parkinson's disease (PwPD) and their informal caregivers. The specific aims for this dissertation, the chapters of the dissertation they are addressed in and the corresponding study project are summarized in Table 1. Enclosed is the methodology for both projects and their aspects that address the specific aims.

Table 1: Specific Aims, Corresponding Chapter and Project Number

\begin{tabular}{|l|c|c|}
\hline Overarching and Specific Aims & Chapter \# & Project \# \\
\hline $\begin{array}{l}\text { Aim 1: To assess the nutrition risk of PwPD overtime. } \\
\text { Track the nutritional status of PwPD overtime } \\
\text { - Examine how disease sequelae may influence nutritional } \\
\text { status. }\end{array}$ & 1 & 1 \\
\hline $\begin{array}{l}\text { Aim 2: Evaluate the nutritional status in PwPD and their } \\
\text { caregivers } \\
\text { - Evaluate the diet quality among PwPD and their informal } \\
\text { caregivers; } \\
\text { Describe the self-reported nutrition concerns among PwPD } \\
\text { and their informal caregivers; } \\
\text { Explore if the nutrition concerns match the dietary quality of } \\
\text { PwPD and informal caregivers. }\end{array}$ & 2 & 2 \\
\hline $\begin{array}{l}\text { Aim 3: Examine PwPD's and their caregivers' perceptions and } \\
\text { acceptance of digital health }\end{array}$ & 3 & 2 \\
\hline $\begin{array}{l}\text { Aim 4: Describe digital competence among PwPD and their } \\
\text { caregivers. }\end{array}$ & 3 & 2 \\
\hline
\end{tabular}




\section{Project 1 Design and Methodology (Aim 1, Chapter 1)}

This is an ancillary study of a five-year observational, longitudinal study assessing the nutritional, cardio-metabolic, cognitive and physical function status of PwPD, acquired brain injury $(A B I)$ and neurotypical participants (Longitudinal Study of Communication, Nutrition and Physical Activity, IRB HU1314-006). Participants come to URI's Speech and Hearing Clinic located at the Kingston Campus and are assessed every six months for five years. Participants are enrolled in the study on a rolling basis. Potential participants aged 18-85 years are recruited via brochures and word-of-mouth; one-year post-PD or -ABI diagnosis. The time commitment for participants is three hours per evaluation for a total of 30 hours for the five years. Written data were stored in a locked file cabinet in the Department of Communicative Disorders. Prior to their first assessments, participants went through the informed consent process (Appendix C).

For Chapter 1, those PwPD who completed an assessment their baseline year, year 2 and year 4 were included in this study. These participants completed their assessments from Fall 2013 through Spring 2019. Table 2 summarizes the research timeline for chapter 1 . Table 3 summarizes the measures performed at each assessment visit. 
Table 2: Specific Aim 1 Study Timeline

\begin{tabular}{|l|c|c|c|c|c|c|c|}
\hline Activities & Fa 2013- & Fa & Sp & Fa & Sp & Fa & Sp \\
\hline Sp 2017 & 2017 & $\mathbf{2 0 1 8}$ & $\mathbf{2 0 1 8}$ & $\mathbf{2 0 1 9}$ & $\mathbf{2 0 1 9}$ & $\mathbf{2 0 2 0}$ \\
\hline Recruit & $X$ & $X$ & & & & & \\
\hline Assessment Visits & $\mathrm{X}$ & & & & & & \\
\hline Data Entry & $\mathrm{X}$ & $\mathrm{X}$ & $\mathrm{X}$ & $\mathrm{X}$ & $\mathrm{X}$ & & \\
\hline Data Analysis & & & & & $\mathrm{X}$ & $\mathrm{X}$ & \\
\hline Manuscript Preparation & & & & & & $\mathrm{X}$ & $\mathrm{X}$ \\
\hline
\end{tabular}

Table 3: Assessment Protocols by Domain

\begin{tabular}{|l|l|}
\hline Assessment Domain & Assessment Tool \\
\hline Nutrition & The Dietary Screening Tool (DST) ${ }^{\star 1}$ \\
\hline Cardiometabolic & Lipid profiles and blood pressure \\
\hline Anthropometric & $\begin{array}{l}\text { Height, weight, body mass index (BMI), percent body } \\
\text { fat }^{*}\end{array}$ \\
\hline Physical Functioning & Short Physical performance Battery (SPPB) $^{2}$ \\
\hline Swallowing & $\begin{array}{l}\text { Swallowing Quality of Life Survey (SWAL-QOL) } \\
\text { Timed Swallow Test }\end{array}$ \\
\hline Cognition & $\begin{array}{l}\text { Repeatable Battery for the Assessment of } \\
\text { Neuropsychological Status (RBANS) }\end{array}$ \\
\hline Medical History & Past Medical History Questionnaire \\
\hline${ }^{*}$ Assessments used to for Aim1 \\
\hline
\end{tabular}

\section{Data Collection}

\section{Nutritional Assessment Measures}

Within the nutrition assessment, the dietary screening tool was used to assess nutrition risk, and biochemical, clinical, and anthropometric data were obtained Participants completed the dietary screening tool (DST, Appendix D), a 25-item questionnaire validated and used to identify dietary patterns and nutritional risk in older adults. ${ }^{1,6}$ A total of 105 points can be achieved. Scores can be categorized as: at risk (<60), possible risk (60-75), and not at nutrition risk (>75). The eight component scores from the DST were also examined, which include: whole fruit and juice (15 points), vegetables (15 points), whole grains (15 points), lean protein (10 points), processed meats (10 points), dairy 
(10 points), added fats, sugars and sweets ( 25 points), and supplements (5 points). We determined participants who achieved $\geq 80 \%$ for each DST component score.

Biochemical and Clinical Data (Appendix E). Serum total cholesterol, low-density lipoprotein cholesterol, high-density lipoprotein cholesterol, triacylglycerol, and glucose will be obtained using a finger stick (Cholestech ${ }^{\circledR}$ LDX system, Hayward, CA) after a 12-hour fast. Blood pressure will be measured using automatic blood pressure machine (Omron Healthcare Inc., Bannockburin, IL).

Anthropometrics. Height was measured using a stadiometer (Seca, Birmingham, UK) to the nearest $0.5 \mathrm{~cm}$ in duplicate and then averaged. Measures were repeated if obtained height was not within $0.2 \mathrm{~cm}$ of each other. Weight was obtained using a calibrated electronic scale (Healthometer 752KL, Jarden Consumer Solutions, Boca Raton, FL) to the closest $0.1 \mathrm{~kg}$. Weight measurements were taken in duplicate and averaged. The average height and weight value were used to calculate body mass index (BMI, $\mathrm{kg}$ of body weight/height in meters ${ }^{2}$ ). A BMI between 28 and 23 was considered within an optimal range, aligning with older adults. ${ }^{7}$

\section{Other measures}

The measures described below were collected at each assessment visit and analyzed to help better understand and explore factors that may influence nutritional status among PwPD. 
Physical Functioning. The SPPB (Appendix F) assessed participants' global physical functioning and includes gait speed, standing balance tests, and five repeated chair stands. ${ }^{2}$ Scores $<10$ indicated mobility disability. ${ }^{2}$

Cognitive Evaluation. To measure attention, language, memory, constructional and visual-spatial abilities, the RBANS (Appendix G) will be used. ${ }^{5}$ The test includes 12 subtests that can be completed in 30 minutes. A score $<80$ indicates cognitive impairment may be present.

Swallowing Evaluation. The SWAL-QOL (Appendix $\mathrm{H}$ ) is a 44-item survey that takes 10 minutes to complete. Participants rate factors about 10 quality of life concepts related to swallowing. ${ }^{3} A$ timed swallow test $(\mathrm{ml} / \mathrm{s})$ is administered to assess strength, coordination, range of movement, and timing of movement of swallowing muscles in those with neurological disorders (Appendix I). ${ }^{4}$ The number of swallows and the time it takes to swallow the water are counted.

Medical History. A medical history questionnaire (Appendix $\mathrm{J}$ ) is used to identify health-related conditions and takes ten minutes to complete.

Statistical Analysis. Data was analyzed in SPSSv26. Categorical variables were represented as numbers and percentages and continuous variables were reported as mean \pm standard deviations. Data was assessed for normality and non-normally distributed data was transformed for analyses but original mean \pm standard deviations were reported. To assess changes in nutritional risk via DST scores, a repeated measures analysis of covariance was completed with time since diagnosis as a covariate. A Bonferroni 
adjustment was made for multiple comparisons. Analyses were 2-tailed and a $p<0.05$ will indicate statistical significance Paired sample t-tests were also conducted among outcome variables from baseline to year 2, year 2 to year 4 and baseline to year 4 were also conducted. Analyses were 2-tailed and a $p<0.05$ will indicate statistical significance. Participants were then individually analyzed for suboptimal scores across each outcome variable and the frequency of sub-optimal scores were reported.

\section{Project 2 (Chapters 2 \& 3, Aims 2-4)}

\section{Project 2 Overview}

Chapters 2and 3 were from a descriptive, cross-sectional study collected qualitative and quantitative data from PwPD $(n=20)$ and their informal caregivers $(n=20)$ via dyadic semi-structured interviews $(n=20)$, questionnaires, and anthropometric assessment. The project was approved by the Institutional Review Board (HU1819-001, Telenutrition and Parkinson's disease). The purpose of project 2 was to examine the perception and acceptance of digital health technologies in PwPD and their caregivers, as well as understand facilitators and barriers for digital health adoption for the nutrition care process. This study also evaluated the nutritional status and digital competence in PwPD and caregivers. A concurrent mixed-method design $^{8}$ was used to assess the nutritional status and nutrition concerns of PwPD and their informal caregivers. This mixed-methods design was chosen to gain a more complete understanding of nutritional needs of PwPD and caregivers through comparing and synthesizing both quantitative and 
qualitative data. ${ }^{8}$ Data was collected over four assessments completed between November 2018 and April 2019. A summary of activities that occurred in each session is provided in Table 4. Sessions 1, 3, and 4 were conducted by phone, while Session 2 was conducted in the participants' home. The study timeline is summarized in Table 5.

Table 4: Aims 2-4 Proposed Study Timeline

\begin{tabular}{|l|c|c|c|c|c|c|}
\hline Activities & Fall 2017 & $\begin{array}{c}\text { Su } \\
\mathbf{2 0 1 8}\end{array}$ & $\begin{array}{c}\text { Fall } \\
\mathbf{2 0 1 8}\end{array}$ & $\begin{array}{c}\text { Spr } \\
\mathbf{2 0 1 9}\end{array}$ & $\begin{array}{c}\text { Fall } \\
\mathbf{2 0 1 9}\end{array}$ & $\begin{array}{c}\text { Spr } \\
\mathbf{2 0 2 0}\end{array}$ \\
\hline Proposal Defense & $\mathrm{X}$ & & & & & \\
\hline IRB & & $\mathrm{X}$ & & & & \\
\hline $\begin{array}{l}\text { Recruit and } \\
\text { Consent }\end{array}$ & & $\mathrm{X}$ & $\mathrm{X}$ & & & \\
\hline Assessment Visits & & $\mathrm{X}$ & $\mathrm{X}$ & $\mathrm{X}$ & $\mathrm{X}$ & \\
\hline Data Entry & & $\mathrm{X}$ & $\mathrm{X}$ & $\mathrm{X}$ & $\mathrm{X}$ & \\
\hline Data Analysis & & & & $\mathrm{X}$ & $\mathrm{X}$ & \\
\hline $\begin{array}{l}\text { Manuscript } \\
\text { Preparation }\end{array}$ & & & & & $\mathrm{X}$ & $\mathrm{X}$ \\
\hline Defend Dissertation & & & & & & $\mathrm{X}$ \\
\hline
\end{tabular}

Table 5: Description and Timeline of Evaluations for Project 2

\begin{tabular}{|l|l|l|l|}
\hline Session & Location & Activities & Time \\
\hline $\begin{array}{l}\text { Session } \\
1\end{array}$ & $\begin{array}{l}\text { Phone } \\
\text { call }\end{array}$ & $\begin{array}{l}\text { Informational Phone Call; Cognitive } \\
\text { Screening }\end{array}$ & 30 minutes \\
\hline $\begin{array}{l}\text { Session } \\
2\end{array}$ & In-person & $\begin{array}{l}\text { Informed Consent; Questionnaires; } \\
\text { Anthropometrics, Timed Swallow Test; Semi- } \\
\text { Structured Interviews }\end{array}$ & 125 minutes \\
\hline $\begin{array}{l}\text { Session } \\
3\end{array}$ & $\begin{array}{l}\text { Phone } \\
\text { call }\end{array}$ & $1^{\text {st }}$ 24-hour Dietary Recall & 60 minutes \\
\hline $\begin{array}{l}\text { Session } \\
4\end{array}$ & $\begin{array}{l}\text { Phone } \\
\text { call }\end{array}$ & $2^{\text {nd }}$ 24-hour Dietary Recall & 60 minutes \\
\hline & & Total Participant Time & $\mathbf{2 7 5}$ minutes \\
\hline
\end{tabular}

Assessment 1 was an informational phone call during which participants were screened for eligibility and informed about the study protocol. At the beginning of Assessment 2, the study protocol was reviewed and both PwPD and their informal caregiver completed the informed consent process. 
Assessment 2 was an in-person home visit, which consisted of a timedswallow screening, questionnaires, and a semi-structured, dyadic interview. Assessments 3 and 4 were phone calls during which participants completed two 24-hour recalls.

Theoretical Framework. This mixed-methods study targeted elements of the emerging middle-range theory of transitions.$^{9} \mathrm{~A}$ transition represents change from one state or condition to another, and includes life development stages, such becoming an informal caregiver. Research shows helping informal caregivers acquire new skills to cope with situations related to caregiving, and creating feelings of informal support for caregivers, plays a central role in successful role transition. ${ }^{9}$ Acquisition of subjective and objective data from PwPD and caregivers was collected to help better understand the transition dyads face as the patient-caregiver relationship changes, and the role of the caregiver evolves ${ }^{9}$, especially in relation to dietary management. The approach has been used to provide tailored health interventions for those with neurodegenerative diseases and their caregivers. ${ }^{10,11}$

This study concentrated on the early stages of digital health nutrition services, in which PwPD and caregivers provided personal opinions and preferences to inform the creation of an user-friendly, evidenced-based, digital health service. ${ }^{12}$ Formative analysis has been used to effectively employ digital health technologies for older adults ${ }^{13,14}$, and in the development of dyadic interventions for PwPD. ${ }^{10}$ Findings from the proposed study can be incorporated into the technology acceptance model (TAM). ${ }^{15}$ 
Inclusion Criteria. Both the PwPD and their informal caregiver were required to participate and needed to be community-dwelling, English speaking, and $\geq 18$ years old. Participants also needed to score $\geq 18$ on the Telephone Montreal Cognitive Assessment (T-MoCA), a cognitive screening tool, that was administered during Assessment 1. ${ }^{16,17} \mathrm{~A}$ list of inclusion criteria for both PwPD and caregivers are summarized in Table 6 .

Table 6: Inclusion Criteria for Project 2 (Specific Aims 2-4)

\begin{tabular}{|l|l|}
\hline PwPD & Informal Caregiver \\
\hline $18+$ years old & $18+$ years old \\
\hline English Speaking & English Speaking \\
\hline Cognitive Function $<18$ on t-MoCA & Cognitive Function $<18$ on t-MoCA \\
\hline Has identified an informal caregiver & Performs majority of care \\
\hline
\end{tabular}

Recruitment and Data Saturation. Recruitment occurred following study approval by URl's Institutional Review Board (HU1819-001). Emails, flyers (Appendix K), announcements at support groups, word of mouth and current statewide collaborations and contacts were used to recruit participants. Participants were recruited from support groups throughout New England, New York and New Jersey via announcements and flyers at community centers, via healthcare providers, the American Parkinson's Disease Association website, and through popular press coverage from the University.

Twenty-five dyads expressed interest. Five dyads did not continue with the study due to scheduling conflicts or low T-MoCA scores. Eighteen dyads were eligible, enrolled and interviewed. Two of these dyads included couples who were both living with PD and identified as each other's informal caregivers and were double counted as a PwPD and a caregiver. As a result, 20 dyads 
were included in analyses. Dyads were from Rhode Island, Massachusetts, New York, and Connecticut. We aimed to interview up to 20 dyads as previous research among PwPD and caregivers indicates this is where data saturation is reached. ${ }^{18,19}$ Saturation was reached after the fourteenth interview.

Incentive Structure. Dyads who complete all 4 sessions will receive tailored dietary recommendations based on individual assessments, and a chance to win a wearable device to monitor health. Emerging themes and general findings from interviews related will be provided

Informed Consent Process. Potential participants will receive an informational phone call (session 1) to review the study purpose and requirements. Consent was provided or declined from the PwPD and their informal caregiver at the beginning of the in-person evaluations (session 2). Both the caregiver and the PwPD were consented. Interested and eligible dyads were enrolled in the study. (Appendix $L \& M$ ). Enrolled dyads will complete a demographic and medical history questionnaire, then the semistructured interview. During the informed consent, there was a request authorization for disclosure of protected health information from primary care physicians or neurologists for PwPD, which included: PD stage, date of diagnosis, current medications and treatment. Permission to request personal health information from PwPD's neurologist was also obtained (Appendix X), in order to obtain information regarding disease staging. 


\section{Collection and Analyses of Qualitative and Quantitative Data Qualitative Assessment and Analysis.}

Semi-structured Dyadic Interviews. A 24-question moderator guide, informed by the previous literature and the research team was organized to capture three main domains: PD and Diet, Accessibility of Nutrition and Health Information, and Digital Health for PD. From these domains, participants' acceptance and perception were assessed. A copy of the moderator guide is provided in Appendix L. The moderator guide included three key sections of the moderator's script: 1.) PD and Diet, 2.) Accessibility of Nutrition and Health Information, 3.) Digital Health for PD. Prior to starting the study, interviews were piloted with two dyads and questions were modified based on participant feedback. Interviews were conducted in the participants' homes (facilitated by $\mathrm{DL}$ ) and were audio-recorded using a digital recorder. The mean interview duration was 39 minutes (range 21-64 minutes). During interviews, participants were provided operational definitions of terms (technology, digital health, smart phones, smart watches, apps, videoconferencing). Photo prompts were used to help describe different technological devices and digital health tools and this was particularly important for understanding acceptance of devices.

Qualitative analyses related to digital health specific aims (Aim 3):

Qualitative data was analyzed using deductive and inductive reasoning. Transcripts were deductively-coded using the framework analysis method ${ }^{20}$, a seven-stage, systematic approach used in healthcare research. Transcripts 
were inductively-coded using Colaizzi's Strategy in Descriptive

Phenomenology ${ }^{21}$; this interpretative approach draws an understanding of participants' "lived experiences". ${ }^{22}$

The following steps were taken to analyze the data related to perception and acceptance of digital health, facilitators and barriers and identified features for a digital health service. Recordings were transcribed verbatim and DL checked transcripts for accuracy (stage 1). Transcripts were divided into three batches. DL and a trained research assistant (KS) analyzed one batch at a time. Separately, DL and KS coded for the following a priori themes related to digital health: perception (perceived usefulness, perceived ease of use, awareness of digital health, image of technology) and acceptance (accept, neutral, reject). These a-prior themes adopts components of the technology acceptance model (TAM) ${ }^{15}$

Before coding individually, KS and DL read through an entire batch of transcripts, met to discuss initial impressions, developed a list of initial themes and then coded a-priori (stage 2). Both DL and KS coded one transcript from the batch independently and in duplicate (stage 3). The two researchers compared and reconciled coding, and there was a strong agreement between authors on the transcripts reviewed. During this discussion the two researchers developed a working analytical framework and agreed upon which codes to use on the remaining transcripts (stage 4). The transcripts were uploaded to NVivo12 (QSR International Pty Ltd, AU) and coded separately by DL and KS. An inter-coder reliability was calculated and found acceptable ${ }^{23}$, 
with an agreement $>80 \%$ achieved for each overarching theme across each batch. DL and KS met to reconcile coding differences. The research committee and DL met to collapse and finalize themes (stage 5). Data was then charted into framework matrices using NVivo12 to display codes within each theme (stage 6). Data was interpreted, and connections related to digital competence and technological preferences of PwPD and their caregivers were made (stage 7). Although a priori themes helped to inform this framework, a phenomenological and iterative approach throughout each stage of the analyses was also taken to identify emerging themes that may impact technology use among this population. Both DL and KS contributed to the framework development with the advisement of the dissertation committee.

\section{Qualitative Analyses Related to Nutrition (Aim 2):}

The two coders ( $\mathrm{DL}$ and $\mathrm{KS}$ ) took a similar approach to analyze the interviews for nutrition concerns. Before coding individually, KS and DL read through all of the transcripts and developed a list of initial impressions and themes, which fell under three categories: Dietary Concerns Related to PD Sequelae, Other Nutrition Concerns and Perceptions of Diet. Both DL and KS coded one transcript from each batch independently and met to compare and reconcile coding. At this point $\mathrm{DL}$ and $\mathrm{KS}$ created a working analytical framework and agreed upon codes to use for analyzing the remaining transcripts. The transcripts were uploaded to NVivo12 (QSR International Pty Ltd, AU) and coded separately by $\mathrm{DL}$ and KS. An inter-coder reliability was calculated, with an agreement $>80 \%$ achieved. ${ }^{23}$ 
$\mathrm{DL}$ and KS compared codes between each batch and reconciled differences between codes, until a consensus was reached. Codes were then finalized. To ensure information related to dietary intake was not overlooked in other sections of the transcripts, DL went through all of the transcripts and coded the remaining sections. KS reviewed these codes to verify coding structure, added additional codes when needed and DL and KS discussed differences in coding and collapsed themes. The larger research team and DL met to further collapse and finalize themes.

\section{Quantitative Data Collection and Analyses.}

The following assessments will be completed and analyzed for both PwPD and their informal caregiver, unless otherwise noted.

Dietary Data. Nutrition Risk and Diet Quality.

Within the nutrition assessment, the dietary screening tool was used to assess nutrition risk, and biochemical, clinical, and anthropometric data were obtained Participants completed the dietary screening tool (DST, Appendix D), a 25-item questionnaire validated and used to identify dietary patterns and nutritional risk in older adults. ${ }^{1,6}$

Both PwPD and caregivers completed two 24-hour recalls via telephone (sessions 3 and 4). These recalls included one weekend and week day, and were conducted using the gold-standard, multiple-pass interview method (Appendix O).${ }^{24}$ Participants received a food amounts booklet to estimate and report accurate portion sizes. Twenty-four-hour recalls were entered into Nutrition Data System for Research Software 2017 (NDSR, University of 
Minnesota, Minneapolis, MN) and analyzed for total energy, food group and nutrient intake. The NDSR data was assessed for diet quality using the Healthy Eating Index (HEI)-2015 scoring metric. ${ }^{25}$ Total scores can range from 1-100; the higher the score, the better the diet quality. Mean scores from the two days were used. Outputs from NDSR were used to calculate HEI-2015 component scores (whole grains, whole fruits, total fruits, vegetable, greens and beans, dairy, total proteins, seafood, plant protein and seafood, refined grains, sodium, added sugars, and saturated fat) derived using USDA SAS codes ${ }^{26}$ Component scores help to provide insight into types of food consumed and nutrients of interest for future interventions.

Anthropometrics. Height was measured using a stadiometer (Seca, Birmingham, UK) to the nearest $0.5 \mathrm{~cm}$ in duplicate and then averaged. Measures were repeated if obtained height was not within $0.2 \mathrm{~cm}$ of each other. Weight was obtained using a calibrated electronic scale (Tanita HD351, Japan) to the closest $0.1 \mathrm{~kg}$. Weight measurements were taken in duplicate and averaged. The average height and weight value were used to calculate body mass index (BMI, $\mathrm{kg}$ of body weight/height in meters $\left.{ }^{2}\right)$. A BMl between 28 and 23 was considered within an optimal range, aligning with older adults. ${ }^{7}$

The bioelectrical impedance analysis (InBody, Korea) device ${ }^{27}$, assessed body composition ${ }^{28,29}$, and has been used with PwPD. ${ }^{30}$ The analysis takes five minutes to complete. Body composition was not be obtained for participants with implantable medical devices. Guidelines proposed by the American Council on Exercise were used to assess percent 
body fat ${ }^{31}$, with values of $<32 \%$ for women and $<25 \%$ for men considered acceptable. ${ }^{31}$ Standardized protocol was used to measure waist circumference with Gulick anthropometric tape (Fabrication Enterprises Inc. White Plains, NY). ${ }^{32}$ Waist circumference was measured in the horizontal plane at the superior border of the iliac crest. Measurements were taken in duplicate and averaged.

Digital Competence and Technology Use. In addition to digital health data gathered during semi-structured interviews, questions related to technology competence, technology use and digital health use will be gathered through two questionnaires. Questions will be adopted from "Measuring Digital Health Skills across the EU: EU Wide Indicators of Digital Competence," (Appendix $\mathrm{P})^{33}$ which assessed use and level of comfort using various aspects of technology via 15 questions/statements. ${ }^{33}$ For each statement, participants responded strongly disagree, slightly disagree, slightly agree or strongly agree. We also collected data regarding device use, frequently and purposes of device use.

Digital health was assessed using questions from the "2015 Health Information National Trends Survey", Section B (Appendix Q), which contains 11 yes/no questions, as well as questions describing technology use and how health information is accessed. ${ }^{34-36}$ Questions from this questionnaire also asked participants where they go first to search for health information, rate the amount of effort it took to find the information and the describe the confidence in finding quality health information. Questions also asked PwPD and 
caregivers to rate the level of trust they had for obtaining nutrition and health information from an array of sources, including doctors, other health professions, family and friends, organizations and online blogs.

Other Measures. Assessment tools measured cognition, anthropometrics, disease related QOL, caregiver burden, swallowing function, medical history, and demographics. These variables were used to help describe participants.

Cognitive Status. Cognitive status was assessed using the T-MoCA (Appendix R). ${ }^{16,17}$ The test takes about 10 minutes to complete and is validated in community-dwelling adults with mild cognitive impairment. ${ }^{17}$ It includes eight subtests assessing digit span, attention, calculation, repetition, verbal fluency, abstraction, recall and orientation. ${ }^{16}$ Participant who have less than 12 years or less of formal education will receive one point toward their total score. Scores $<18$ out of a possible 22 points indicate mild cognitive impairment.

Swallowing Function. A timed swallow test $(\mathrm{ml} / \mathrm{s})$ was administered to assess swallowing muscles in those with neurological disorders. ${ }^{4}$ Specifically, it assesses: strength, coordination, range of movement and timing of movement of swallowing muscles (Appendix I). The Eating Assessment Tool $(\text { EAT-10 })^{37}$, a validated 10 -item questionnaire, screened for self-reported swallowing difficulties (Appendix S). Scores on the EAT-10 range from 0-40; higher scores indicate a swallowing problem.

Disease Related Quality of Life. The 39-item Parkinson's Disease Questionnaire (PDQ-39) is a disease-specific tool that assessed the health- 
related quality of life of PwPD (Appendix T). ${ }^{38,39}$ The questionnaire contains eight sub-scales (mobility, emotional well-being, stigma, social support, cognitions, communication and bodily discomfort), which were used to calculate global health-related quality of life. Each item scores from 0 (never) to 4 (always), with the highest possible score of 156, and higher scores indicate worse quality of life. ${ }^{38,39}$ Results are reported using an index percentage $(0 \%=$ no disability and $100 \%=$ maximum disability $)$.

Caregiver Burden. The Multidimensional Caregiver Strain Index (MCSI) assessed caregiver burden. ${ }^{40}$ This 18 -item tool collected subjective information from informal caregivers across six domains: physical strain, social constraints, financial strain, time constraints, interpersonal strain, and elder demanding/manipulative. Response to individual questions are reported on a 0 (never) to 4 (a great deal) scale. Scores range from 0-64 points and are reported as an index percentage ${ }^{130}$; higher scores indicate higher caregiver strain $^{130}$. The scale has been previously used successfully in the PD population $^{131}$.

Medical History and Demographics. To identify health-related conditions and past medical history, a non-validated medical history questionnaire was used. Topics covered included: questions related to neurological, endocrine and cardiovascular and gastrointestinal health. Demographics (age, gender, race/ethnicity, employment status) were collected. Health information and demographic information was obtained from both PwPD and their caregivers (Appendix V and W). 
Quantitative Statistical Analyses. Quantitative data was analyzed using SPSS v26 (IBM Corp, Summers, NY). Descriptive statistics are reported as mean \pm standard deviation for continuous variables and percentages and frequencies for categorical variables. Data was examined for outliers, and outliers were found among the following HEI-2015 variables: HEI-2015 Total Scores, Protein, and Refined Grains component scores. Independent t-tests explored differences between PwPD and caregivers for all normally distributed continuous variables. A Mann-Whitney $U$ test examined group differences between non-normally distributed continuous variables. A chi-square analysis explored differences for categorical variables. A p-value of $<0.05$ indicated statistical significance. Finally, the percent and frequency of PwPD and caregivers who met $>80 \%$ of adequacy and moderation HEI-2015 components scores were calculated.

\section{Data Integration.}

Manuscript 2: The research team reviewed both data sets and considered the themes coded from the qualitative research in conjunction with the findings from the statistical analyses of the HEI-2015 scores. Qualitative data was mapped into framework matrices and reported as frequencies using NVivo12 to quantify codes within the over-arching category of Dietary Concerns Related to PD Sequelae. This frequency data stemmed from the qualitative themes and a side-by-side comparison of themes were compared with individual HEI-2015 Scores for each participant. Data was interpreted for 
consistent patterns between HEI-2015 scores and frequency of self-reported Dietary Concerns Related to PD sequelae from PwPD and their caregivers were made.

Manuscript 3: Acceptance of digital health was analyzed by assessing current technology use and purpose from questionnaires and through themes coded from qualitative interviews. Phrases/sentences from qualitative interviews related to acceptance were categorized as Accept, Neutral or Reject. To calculate average acceptance rate among qualitative interviews, the number of phrases coded as Accept, Neutral or Reject were counted and totaled. The total number of phrases coded as Accept were divided by the total number of phrases coded across the three acceptance categories to calculate acceptance rates among each dyad. The percentages were averaged to calculate an average acceptance rate $(n=20)$. Codes from the qualitative interviews were transformed into variables and reported as percent and frequencies. Side-by-side table displays of frequencies derived from perceptions and acceptance (qualitative data) and digital competence scores (quantitative data) were created and interpreted to better describe the population and readiness for a digital health intervention. A Pearson correlation was used to explore if there was an association between the percentage of phrases coded as hard to use and the mean average acceptance rates.

\section{Resources Required and Utilized:}


Partial funding for Project 1 came from the URI Spark Grant and Enhancement for Graduate Research Award. There was no funding for Project 2. Data collection for Project 1 took place in Independence Square in the URI Speech and Hearing Clinic. Data for Project 2 was collected through phone calls and home visits. Department equipment included: stadiometer, bioelectric impedance scale, electronic scale, cholestech analyzers, portable automatic blood pressure machines, and measuring tape. Data was analyzed using software found on the lab computers in the Lipid Metabolism Lab, including Nvivo 12, SPSS, SAS and NDSR. 


\section{LITERATURED CITED:}

1. Bailey RL. Dietary screening tool identifies nutritional risk in older adults. Am J Clin Nutr. 2009;90:177-183.

2. Guralnik JM, Simonsick EM, Ferrucci L, et al. A short physical performance battery assessing lower extremity function: association with self-reported disability and prediction of mortality and nursing home admission. Journal of Gerontology. 1994;49(2):M85-94.

3. McHorney CA, Robbins J, Lomax K, et al. The SWAL-QOL and SWALCARE outcomes tool for oropharyngeal dysphagia in adults: III.

Documentation of reliability and validity. Dysphagia. 2002;17(2):97-114.

4. Nathadwarawala KM, Nicklin J, Wiles CM. A timed test of swallowing capacity for neurological patients. J Neurol Neurosurg Psychiatry. 1992;55(9):822-825.

5. Randolph C. Repeatable Battery for Assessment of Neuropyschological Status. In. Examiner's Manual San Antonio, TX: The Psychological Corporation 1998.

6. Bailey RL, Mitchell DC, Miller CK, et al. A dietary screening questionnaire identifies dietary patterns in older adults. J Nutr. 2007;137:421-426.

7. Winter JE, Maclnnis RJ, Wattanapenpaiboon N, Nowson CA. BMI and all-cause mortality in older adults: a meta-analysis. Am J Clin Nutr. 2014;99(4):875-890.

8. Leavy P. Mixed Methods Research Design. In: Research Design: Quantitative, Qualitative, Mixed Methods, Arts-Based, and CommunityBased Participatory Research Approaches. New York, NY: The Guilford Press; 2017:175-176.

9. Meleis AI, Sawyer LM, Im EO, Hilfinger Messias DK, Schumacher K. Experiencing transitions: An emerging middle-range theory. Adv Nurs Sci. 2000;23(1):12-28. 
10. Beaudet L, Ducharme F, L'Ecuyer N, Chouinard S, Jodoin N, Panisset $M$. Development and evaluation of a dyadic intervention for elderly couples living with moderate-stage Parkinson disease. Applied Nursing Research. 2015;28(4):e21-27.

11. Ducharme FC, Levesque LL, Lachance LM, et al. "Learning to become a family caregiver" efficacy of an intervention program for caregivers following diagnosis of dementia in a relative. The Gerontologist. 2011;51(4):484-494.

12. Cherry $\mathrm{C}$, Macredie RD. The importance of context in information system design: An assessment of participatory design. Requirements Engineering. 1999;4(2):103-114.

13. Swallow D, Petrie H, Power C, Lewis A, Edwards AD. Involving older adults in the technology design process: A case study on mobility and wellbeing in the built environment. Studies in Health Technology and Informatics. 2016;229:615-623.

14. van Velsen L, Illario M, Jansen-Kosterink S, et al. A community-based, technology-supported health service for detecting and preventing frailty among older adults: A participatory design development process. Journal of Aging Research. 2015;2015:216084.

15. Davis FD, Bagozzi RP, Warshaw PR. User acceptance of computer technology: A comparison of two theoretical models. Manage Sci. 1989;35(8):982-1003.

16. Castanho TC, Amorim L, Zihl J, Palha JA, Sousa N, Santos NC. Telephone-based screening tools for mild cognitive impairment and dementia in aging studies: A review of validated instruments. Frontiers in Aging Neuroscience. 2014;6:16.

17. Pendlebury ST, Welch SJ, Cuthbertson FC, Mariz J, Mehta Z, Rothwell PM. Telephone assessment of cognition after transient ischemic attack and stroke: Modified telephone interview of cognitive status and telephone Montreal Cognitive Assessment versus face-to-face Montreal Cognitive Assessment and neuropsychological battery. Stroke. 2013;44(1):227-229. 
18. Boersma I, Jones J, Carter J, et al. Parkinson disease patients' perspectives on palliative care needs: What are they telling us? Neurology Clinical practice. 2016;6(3):209-219.

19. Zizzo N, Bell E, Lafontaine AL, Racine E. Examining chronic care patient preferences for involvement in health-care decision making: The case of Parkinson's disease patients in a patient-centred clinic. Health Expectations. 2017;20(4):655-664.

20. Gale NK, Heath G, Cameron E, Rashid S, Redwood S. Using the framework method for the analysis of qualitative data in multidisciplinary health research. BMC Med Res Methodol. 2013;13:117.

21. Shosha GA. Employment of Colaizzi's Strategy in Descriptive Phenomenology: A Reflection of a Researcher. European Scientific Journal. 2012;8(27):31-43.

22. Hycner RH. Some guidelines for the phenomenological analysis of interview data. Human Studies. 1985;8(3):279-303.

23. Saladana J. The Coding Manual for Qualitative Researchers. Thousand Oaks, California Sagem 2016.

24. Johnson RK, Driscoll P, Goran MI. Comparison of multiple-pass 24hour recall estimates of energy intake with total energy expenditure determined by the doubly labeled water method in young children. Journal of the American Dietetic Association. 1996;96(11):1140-1144.

25. Guenther PM, Casavale KO, Reedy J, et al. Update of the Healthy Eating Index: HEl-2010. Journal of the Academy of Nutrition and Dietetics. 2013;113(4):569-580.

26. National Institute of Neurological Disorders and Stroke. NINDS Deep Brain Stimulation for Parkinson's Disease Information Page. 2012; http://www.ninds.nih.gov/disorders/deep_brain_stimulation/deep_brain_ stimulation.htm. Accessed December 2, 2013. 
27. Chien MY, Huang TY, Wu YT. Prevalence of sarcopenia estimated using a bioelectrical impedance analysis prediction equation in community-dwelling elderly people in Taiwan. Journal of the American Geriatrics Society. 2008;56(9):1710-1715.

28. Barichella M, Pinelli G, lorio $L$, et al. Sarcopenia and dynapenia in patients with Parkinsonism. Journal of the American Medical Directors Association. 2016;17(7):640-646.

29. Ling $\mathrm{CH}$, de Craen AJ, Slagboom PE, et al. Accuracy of direct segmental multi-frequency bioimpedance analysis in the assessment of total body and segmental body composition in middle-aged adult population. Clinical nutrition. 2011;30(5):610-615.

30. Sartorio A, Malavolti M, Agosti F, et al. Body water distribution in severe obesity and its assessment from eight-polar bioelectrical impedance analysis. European journal of Clinical Nutrition. 2005;59(2):155-160.

31. American Council on Exercise. Percent Body Fat Norms for Men and Women.2013;

http://www.acefitness.org/?creative $=45247336884 \&$ device $=$ c\&network $=$ g\&matchtype=b\&gclid=CNi3ieq6jrOCFcFIOgodCVAAxQ. Accessed June 2, 2013.

32. Janssen I, Heymsfield SB, Allison DB, Kotler DP, Ross R. Body mass index and waist circumference independently contribute to the prediction of nonabdominal, abdominal subcutaneous, and visceral fat. Am J Clin Nutr. 2002;75(4):683-688.

33. European Commission. Measuring digital skills across the EU: EU wide indicators of digital competence. 2014; https://ec.europa.eu/digitalsingle-market/en/news/measuring-digital-skills-across-eu-eu-wideindicators-digital-competence. Accessed January 2, 2017.

34. Kontos E, Blake KD, Chou WY, Prestin A. Predictors of eHealth usage: Insights on the digital divide from the Health Information National Trends Survey 2012. Journal of Medical Internet Research. 2014;16(7):e172. 
35. Nelson DE, Kreps GL, Hesse BW, et al. The Health Information National Trends Survey (HINTS): development, design, and dissemination. J Health Commun. 2004;9(5):443-460; discussion 481444.

36. National Cancer Institute. Health Information National Trends Survey 4 (HINTS 4): HINTS-FDA Methodologies 2015; https://hints.cancer.gov/docs/Instruments/HINTSFDA_Methodology_Report.pdf. Accessed June 30, 2017.

37. Belafsky PC, Mouadeb DA, Rees CJ, et al. Validity and reliability of the Eating Assessment Tool (EAT-10). Ann Otol Rhinol Laryngol. 2008;117(12):919-924.

38. Jenkinson C, Fitzpatrick R, Peto V, Greenhall R, Hyman N. The Parkinson's Disease Questionnaire (PDQ-39): development and validation of a Parkinson's disease summary index score. Age and Ageing. 1997;26(5):353-357.

39. Peto V, Jenkinson C, Fitzpatrick R. PDQ-39: a review of the development, validation and application of a Parkinson's disease quality of life questionnaire and its associated measures. Journal of Neurology. 1998;245 Suppl 1:S10-14.

40. Stull D. The multidimensional caregiver strain index (MCSI): Its measurement and structure. J Clin Geropsychol. 1996;2(11):175-196. 
APPENDIX C: Longitudinal Study Consent Form (Study 1)

Longitudinal Study of Communication, Nutrition and Physical Activity

Leslie A. Mahler, PhD, Principal Investigator

Ingrid Lofgren, $\mathrm{PhD}$, co-Investigator

Matthew Delmonico, PhD, co-Investigator

CONSENT FORM FOR RESEARCH: Participant

Version 5: May 24, 2016

The University of Rhode Island

Department of Communicative Disorders

$25 \mathrm{~W}$ Independence Square, Suite I

Kingston, RI 02881

\section{Purpose of the Consent:}

You have been invited to take part in a research project described below. The purpose of the consent form you are about to read is to provide you with details about the research study and to inform you of your rights if you agree to participate in the study. Your participation is completely up to you. The researcher will explain the project to you in detail. You should feel free to ask questions. If you have more questions later you can call, Dr. Leslie Mahler, the person mainly responsible for this study, at 401874-2490. You may also contact Dr. Ingrid Lofgren at 401-874-5706 or Dr. Matthew Delmonico at 401-874-5440, who are co-Investigators on the study. You must be at least 18 years old and speak English to be in this research project.

\section{Description of the project:}

This is a research project designed to look at communication, nutrition, and physical activity characteristics of adults who have a stroke, traumatic brain injury or Parkinson disease and healthy adults with no known neurological disorder or head injury to be a control group. All evaluations will be conducted at one of two University of Rhode Island locations; in Independence Square on the Kingston Campus at 25 West Independence Way, Kingston or in Independence Square at 500 Prospect Street in Pawtucket.

You are being asked to be in this study because we want to determine the long-term impact of neurological disorders on communication, nutrition, and physical activity. We are looking for 200 people who have a stroke, traumatic brain injury or Parkinson disease to participate in this project. Participation in this study is entirely your choice.

If you decide to take part in this study, you should understand that the evaluations are investigational and you may not experience any benefit from participation.

Participation may also involve additional risks as listed in the Potential Risks and Discomforts section. The consent form will help make sure you understand the tasks 
included in the study before you decide whether you want to take part in the study. You may also quit the study at any time.

\section{What will be done:}

If you agree to take part in this study, you will be asked to complete up to 11 evaluations over five years. Evaluations will take place every six months. The evaluations will include a variety of tasks such as reading sentences and describing a picture, an assessment of how your muscles move, a cognitive screening, an interview, a clinical swallowing evaluation, and questionnaires regarding swallowing, diet and physical activity. The total time for each evaluation will be approximately $3 \frac{1}{2}$ hours. All evaluations will be conducted in a quiet private room at one of the University of Rhode Island Speech and Hearing Clinic locations (Kingston or Pawtucket).

With your permission, we will request health information from your physician about the following specific items only:

- Date of diagnosis

- Current medications

- Imaging information about where the brain damage is located (if appropriate)

- Stage of Parkinson disease (if applicable)

You will sign a separate form to indicate whether you give your permission to release this health information for the study. This information will not be requested for healthy adults enrolled in the study

\section{Potential risks and discomforts:}

There are minimal foreseeable risks associated with these evaluations. There have been no reported adverse affects from clinical evaluation of speech and swallowing. There may be some unknown or unanticipated risks, but every precaution will be taken to ensure your personal safety. Even though experienced personnel will obtain the blood samples from a finger prick, there is a chance of discomfort and minor bruising from the finger stick. For physical function testing there is a risk of muscle soreness or other muscle injury as well as skeletal injury but we will minimize these risks by using standard safety practices.

\section{Purpose and benefits of the study:}

The purpose of this study is to describe communication, nutrition, and physical activity behaviors over time to see how they change and affect quality of life. The information obtained is important because it will help us to understand how to provide services to meet the needs of people with neurological diagnoses. This is an investigational study and there is no guaranteed benefit to your communication or nutrition or physical function as a result of participation in this research study. You will receive personal health information such as your height and weight, physical function determined by a physical assessment, your blood lipids such as cholesterol 
and triglycerides. In addition, you will receive information about your thinking skills and language skills and dietary choices.

\section{Drugs, devices or instruments to be used:}

Drugs will not be used in this study. The equipment for the evaluations include: microphone, sound level meter, tongue blade, a digital tuner, tape recorder, and video cameras. All equipment used to collect cognitive-linguistic and physical function data is considered non-invasive. A lancet and capillary tube will be used to obtain the blood sample from a finger prick and the sample will be analyzed on a small portable machine that is on a table.

\section{Cost to participant:}

There is no cost to you for participation in the evaluations. Parking is available for free.

\section{Confidentiality:}

Your part in this study is confidential. Your individual privacy will be maintained in all published and written data resulting from this study. No names of participants will be published or included in written data resulting from this study. Results of this study may be used for purposes of research, educational lectures, and/or professional presentations. When you are entered into the study you will be assigned a code that does not include any identifying information. For example, the first participant will be coded as Long01. The code number will be used on all response forms and in the analysis of the data.

Dr. Mahler and her research team will have sole access to all contact information and evaluation results containing your name. This information will be kept in a locked filing cabinet in a locked office. However, the U.S. Department of Health and Human Services, and the University of Rhode Island Institutional Review Board have the right to inspect all of your records relating to this research for the purpose of verifying data. Because of the need to release information to these parties, absolute confidentiality cannot be guaranteed. Following completion of this project, contact information will be destroyed for those participants who wish, for any reason, not to be contacted in the future. All other information will be archived and kept in a locked filing cabinet with the study results at the University of Rhode Island. All research data will be retained for a minimum of three years following completion of the study and then will be destroyed. Research data will be located in a locked filing cabinet in the principal investigator's locked office.

Cognitive-linguistic evaluations will be audio and video recorded to allow for data analyses. At times these recordings can be useful for teaching students or professionals about the disorders of people with a neurological diagnosis such as yours. Please indicate by signing below whether you give your permission to use your samples for lectures and presentations. Audio and/or videotapes may be used for teaching for up to 3 years after completion of the study. If you agree, you will never be identified by name in the presentations or lectures. Your decision to give 
permission to use audio and/or video samples in lectures has no impact on your participation in the study.

Yes, I give permission to use audio samples in lectures and presentations.

Yes, I give permission to use video samples in lectures and presentations.

No, I do not want audio samples used except for research

analysis.

No, I do not want video samples used except for research

analysis.

In case there is any injury to you during the study:

If this study causes you any injury, you should immediately contact Dr. Leslie Mahler at (401) 874-2490 or contact the University of Rhode Island Speech and Hearing Clinic at (401) 874-5969. You may also call the office of the Vice President for Research Integrity, 70 Lower College Road, University of Rhode Island, Kingston, RI at (401) 874-4328. If you are injured during an evaluation or during treatment every effort will be made to get you medical attention but you will be responsible for paying for the medical treatment needed.

\section{Decision to quit at any time:}

The decision to take part in this study is up to you. You do not have to participate. If you decide to take part in the study, you may quit and stop participating in this study at any time. You have the right to refuse to answer any question(s) or participate in any procedure for any reason. Deciding not to participate will have no effect on your potential to receive services from a speech-language pathologist. If you wish to quit, simply inform Leslie Mahler at 874-2490 of your decision. If you wish to pursue an alternative treatment instead of completing the study you will be provided with information on how to obtain those services.

\section{Rights and complaints:}

If you are not satisfied with the way this study is performed, you may discuss your complaints with Dr. Leslie Mahler (1mahler@uri.edu; 401-874-2490), Dr. Ingrid Lofgren (ingridlofgren@uri.edu, 401-874-5706), or Dr. Matthew Delmonico (delmonico@uri.edu; 401-874-5440), or you may contact the office of the Vice President for Research for concerns or any questions about your rights as a research subject at: 70 Lower College Road, University of Rhode Island, Kingston, RI at (401) 874-4328 and speak to them anonymously if you choose.

\section{Authorization:}


Your authorization means that you have read this paper and know the purpose of the study and the possible risks and benefits. It also means you know that being in this study is voluntary and you choose to be in this study. You can also withdraw at any time. Your questions have been answered. Your signature on this form means that you understand the information and you agree to participate in this study.

Signature of Participant

Participant Typed/printed Name

Date

Signature of Guardian

Guardian Typed/printed Name

Date
Signature of Researcher

$\overline{\text { Researcher Typed/printed name }}$

Date

Signature of Researcher

$\overline{\text { Researcher Typed/printed name }}$

\section{Please sign both consent forms, keeping one for yourself.}




\title{
APPENDIX D: Dietary Screening Tool
}

\author{
Dietary Screening Tool
}

DIRECTIONS: Please check one response to each question that best describes how you eat.

How often do you usually eat fruit as a snack?

Never

Less than once a week

1 or 2 times a week

3 or more times a week

How often do you usually eat whole grain breads?

Never or less than once a week

1 or 2 times a week

3 or more times a week

How often do you usually eat whole grain cereals?

Never or less than once a week

1 or 2 times a week

3 or more times a week

How often do you usually eat candy or chocolate?

Never

Less than once a week

1 or 2 times a week

3 or more times a week

How often do you eat crackers, pretzels, chips, or popcorn? Never

Less than once a week

1 or 2 times a week

- 3 or more times a week

How often do you eat cakes or pies? Never

Less than once a week

1 or 2 times a week

3 or more times a week 
How often do you eat cookies?

Never

Less than once a week

1 or 2 times a week

3 or more times a week

How often do you eat ice cream?

Never

Less than once a week

1 or 2 times a week

3 or more times a week

How often do you eat cold cuts, hot dogs, lunchmeats or deli meats? Never or less than once a week

1 or 2 times a week

3 or more times a week

How often do you eat bacon or sausage?

Never or less than once a week

1 or 2 times a week

3 or more times a week

How often do you eat carrots, sweet potatoes, broccoli, or spinach?

Never

Less than once a week

1 or 2 times a week

3 or more times a week

How often do you eat fruit (not including juice)? Please include fresh, canned or frozen fruit.

Never or Less than once a week

1 or 2 times a week

3 to 5 times a week

Every day or almost every day

How often do you eat hot or cold breakfast cereal?

Never

Less than once a week

1 or 2 times a week

3 to 5 times a week

Every day or almost every day 
How often do you drink some kind of juice at breakfast?

Never or Less than once a week

1 or 2 times a week

3 to 5 times a week

Every day or almost every day

How often do you eat chicken or turkey?

Never or less than once a week

1 or 2 times a week

More than 3 times a week

How often do you drink a glass of milk?

Never or Less than once a week

1 or 2 times a week

3 to 5 times a week

Every day or almost every day

More than once every day

Do you usually add butter or margarine to foods like bread, rolls, or biscuits?

Yes

No

Do you usually add fat (butter, margarine or oil) to potatoes and other vegetables?

Yes

No

Do you use gravy (when available) at meals?

$$
\text { Yes }
$$

No

Do you usually add sugar or honey to sweeten your coffee or tea?

$$
\text { Yes }
$$

No

Do you usually drink wine, beer or other alcoholic beverages?

Yes

No 
How often do you eat fish or seafood that IS NOT fried? Never

Less than once a week

Once a week

More than once a week

How many servings of milk, cheese, or yogurt do you usually have each DAY?

None

One

Two or more

How many different vegetable servings do you usually have at your main meal of the day?

None

One

Two

Three or more

Which of the following best describes your nutritional supplement use.

I don't use supplements

I use supplements other than vitamins and mineral

I use a multivitamin/mineral preparation (e.g. Centrum) 


\section{APPENDIX E: Biochemical and Clinical Data}

\section{Blood Lipids, Glucose, Blood Pressure Results}

Name

Your Results:

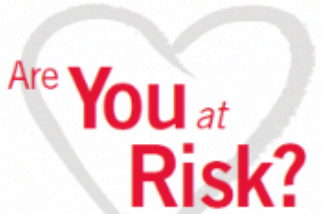

Blood Pressure:

- Desirable: $<120 / 80 \mathrm{mmHg}$

- Borderline high: $\geq 130 / 85 \mathrm{mmHg}$

- High: $\geq 140 / 90 \mathrm{mmHg}$

Total Cholesterol:

- Desirable: $<200 \mathrm{mg} / \mathrm{dL}$

- Borderline: $200-239 \mathrm{mg} / \mathrm{dL}$

- High: $\geq 240 \mathrm{mg} / \mathrm{dL}$

HDL Cholesterol:

- Low: $<40 \mathrm{mg} / \mathrm{dl}$

- High: $\geq 60 \mathrm{mg} / \mathrm{dL}$

LDL Cholesterol:

- Optimal: $<100 \mathrm{mg} / \mathrm{dL}$

- Near optimal/above optimal: $100-129 \mathrm{mg} / \mathrm{dL}$

- Borderline high: 130-159 mg/dL

- High: 160-189 mg/dL

- Very high: $\geq 190 \mathrm{mg} / \mathrm{dL}$

Triglycerides:

- Normal: $<150 \mathrm{mg} / \mathrm{dL}$

- Borderline high: $150-199 \mathrm{mg} / \mathrm{dL}$

- High: $200-499 \mathrm{mg} / \mathrm{dL}$

- Very high: $\geq 500 \mathrm{mg} / \mathrm{dL}$

Glucose:

- Normal fasting glucose: $<100 \mathrm{mg} / \mathrm{dL}$

- Impaired fasting glucose: $100-126 \mathrm{mg} / \mathrm{dL}$

- High fasting glucose: $\geq 126 \mathrm{mg} / \mathrm{dL}$

National Cholesterol Education Program Adult Treatment Panel III Guidelines, American Diabetes Association
Participant ID

Place sticker here

\begin{tabular}{|c|c|}
\hline BP 1 & \\
\hline BP 2 & \\
\hline BP 3 (if necessary) & \\
\hline BP 4 (if necessary) & \\
\hline
\end{tabular}




\section{APPENDIX F: Short Physical Performance Battery}

1.

Balance Tests
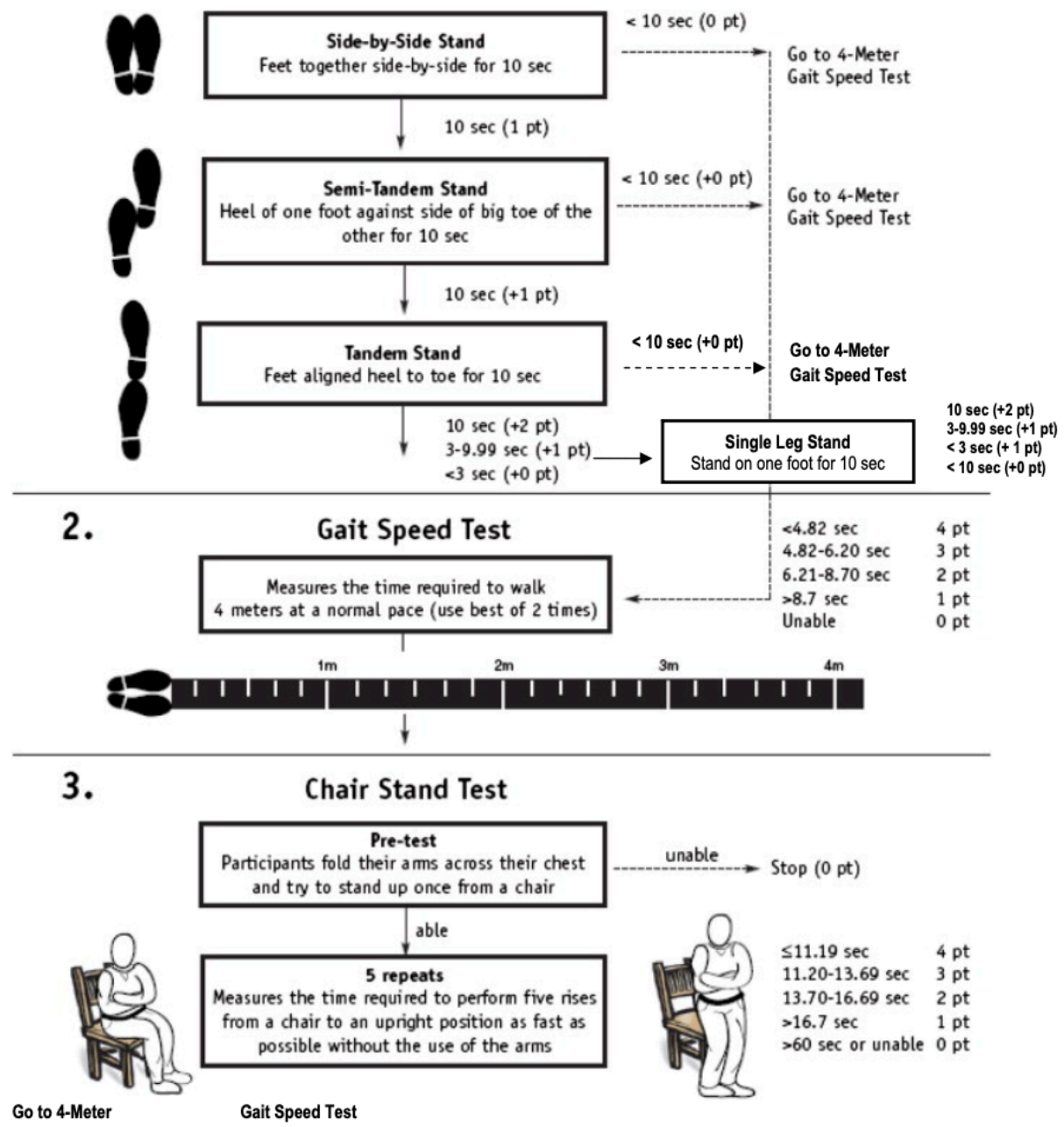


\section{APPENDIX G: Repeatable Battery of the Neuropsychological Status}
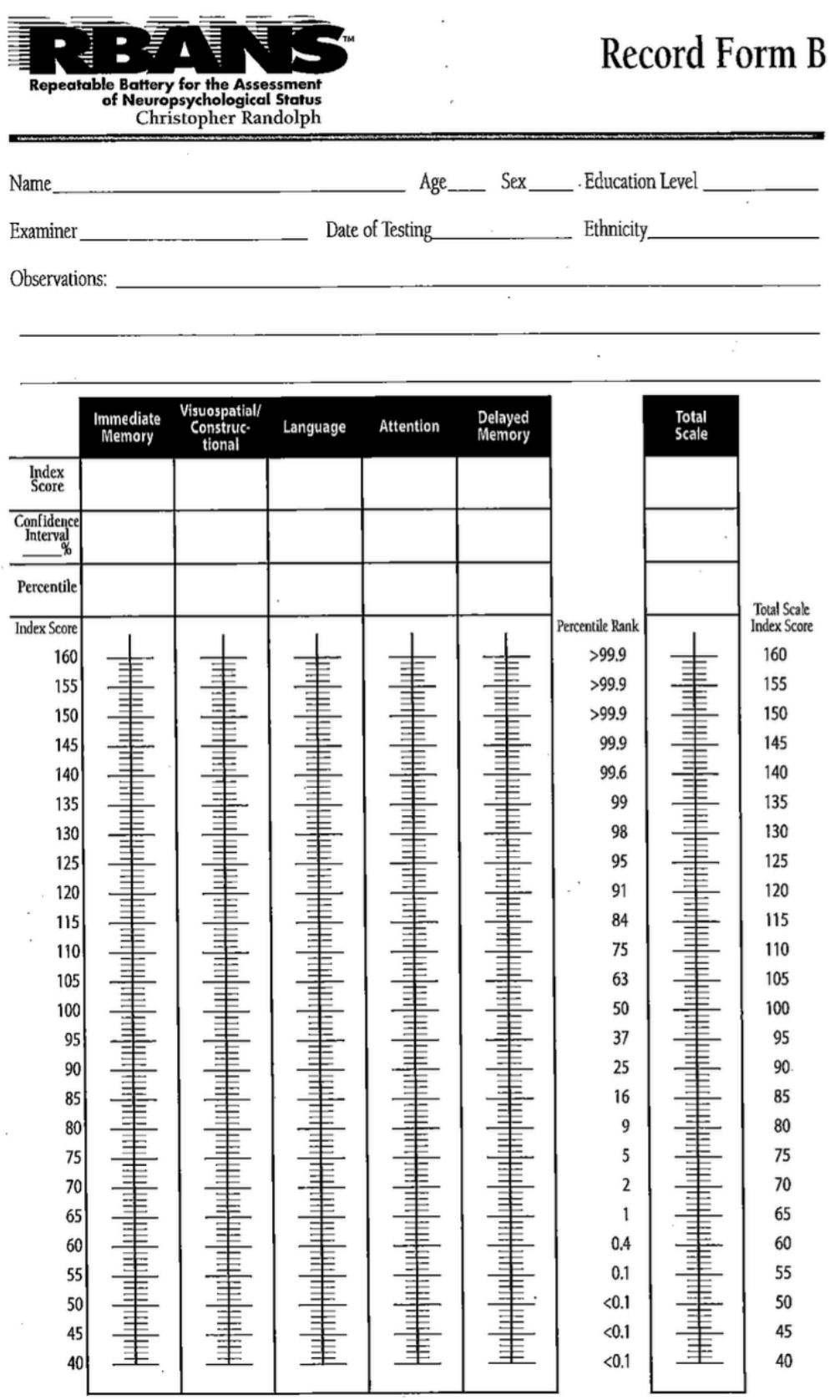

PEARSON

Copyright @ 1998 NCS Pearson, Inc. All rights reserved.

ФPsychCorp

$27282930313233343536 \quad$ C D E 
APPENDIX H: Swallowing Quality of Life Questionnaire

\section{The SWAL-QOL SURVEY}

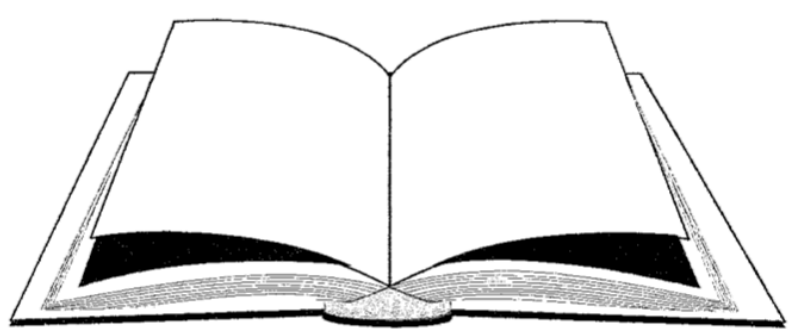

Understanding

Quality of Life

in Swallowing Disorders 


\section{Instructions for Completing the SWAL-QOL Survey}

This questionnaire is designed to find out how your swallowing problem has been affecting your day-to-day quality of life.

Please take the time to carefully read and answer each question. Some questions may look like others, but each one is different.

Here's an example of how the questions in the survey will look.

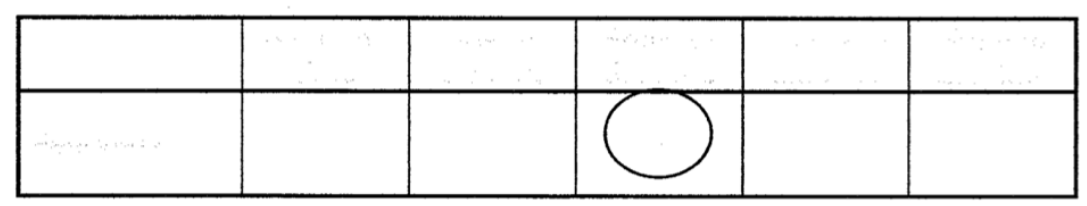

Thank you for your help in taking part in this survey! 
IMPORTANT NOTE: We understand that you may have a number of physical problems. Sometimes it is hard to separate these from swallowing difficulties, but we hope that you can do your best to concentrate only on your swallowing problem. Thank you for your efforts in completing this questionnaire.

1. Below are some general statements that people with swallowing problems might mention. In the last month, how true have the following statements been for you.

(circle one number on each line)

\begin{tabular}{|l|c|c|c|c|c|}
\hline & $\begin{array}{c}\text { Very much } \\
\text { true }\end{array}$ & $\begin{array}{c}\text { Quite a bit } \\
\text { true }\end{array}$ & $\begin{array}{c}\text { Somewhat } \\
\text { true }\end{array}$ & $\begin{array}{c}\text { A little } \\
\text { true }\end{array}$ & $\begin{array}{c}\text { Not at } \\
\text { all true }\end{array}$ \\
\hline $\begin{array}{l}\text { Dealing with my } \\
\text { swallowing problem is } \\
\text { very difficult. }\end{array}$ & 1 & 2 & 3 & 4 & 5 \\
\hline $\begin{array}{l}\text { My swallowing problem is } \\
\text { a major distraction in my } \\
\text { life. }\end{array}$ & 1 & 2 & 3 & 4 & 5 \\
\hline
\end{tabular}

2. Below are aspects of day-to-day eating that people with swallowing problems sometimes talk about. In the last month, how true have the following statements been for you?

(circle one number on each line)

\begin{tabular}{|l|c|c|c|c|c|}
\hline & $\begin{array}{c}\text { Very much } \\
\text { true }\end{array}$ & $\begin{array}{c}\text { Quite a bit } \\
\text { true }\end{array}$ & $\begin{array}{c}\text { Somewhat } \\
\text { true }\end{array}$ & $\begin{array}{c}\text { A little } \\
\text { true }\end{array}$ & $\begin{array}{c}\text { Not at } \\
\text { all true }\end{array}$ \\
\hline $\begin{array}{l}\text { Most days, I don't care if I } \\
\text { eat or not. }\end{array}$ & 1 & 2 & 3 & 4 & 5 \\
\hline $\begin{array}{l}\text { It takes me longer to eat } \\
\text { than other people. }\end{array}$ & 1 & 2 & 3 & 4 & 5 \\
\hline $\begin{array}{l}\text { I'm rarely hungry } \\
\text { anymore. }\end{array}$ & 1 & 2 & 3 & 4 & 5 \\
\hline $\begin{array}{l}\text { It takes me forever to eat } \\
\text { a meal. }\end{array}$ & 1 & 2 & 3 & 4 & 5 \\
\hline $\begin{array}{l}\text { I don't enjoy eating } \\
\text { anymore. }\end{array}$ & 1 & 2 & 3 & 4 & 5 \\
\hline
\end{tabular}


3. Below are some physical problems that people with swallowing problems sometimes experience. In the last month, how often you have experienced each problem as a result of your swallowing problem?

(circle one number on each line)

\begin{tabular}{|l|c|c|c|c|c|}
\hline & $\begin{array}{c}\text { Almost } \\
\text { always }\end{array}$ & Often & Sometimes & $\begin{array}{c}\text { Hardly } \\
\text { ever }\end{array}$ & Never \\
\hline Coughing & 1 & 2 & 3 & 4 & 5 \\
\hline Choking when you eat food & 1 & 2 & 3 & 4 & 5 \\
\hline $\begin{array}{l}\text { Choking when you take } \\
\text { liquids }\end{array}$ & 1 & 2 & 3 & 4 & 5 \\
\hline Having thick saliva or phlegm & 1 & 2 & 3 & 4 & 5 \\
\hline Gagging & 1 & 2 & 3 & 4 & 5 \\
\hline Drooling & 1 & 2 & 3 & 4 & 5 \\
\hline Problems chewing & 1 & 2 & 3 & 4 & 5 \\
\hline $\begin{array}{l}\text { Having excess saliva or } \\
\text { phlegm }\end{array}$ & 1 & 2 & 3 & 4 & 5 \\
\hline Having to clear your throat & 1 & 2 & 3 & 4 & 5 \\
\hline Food sticking in your throat & 1 & 2 & 3 & 4 & 5 \\
\hline Food sticking in your mouth & 1 & 2 & 3 & 4 & 5 \\
\hline $\begin{array}{l}\text { Food or liquid dribbling out of } \\
\text { your mouth }\end{array}$ & 1 & 2 & 3 & 4 & 5 \\
\hline $\begin{array}{l}\text { Food or liquid coming out } \\
\text { your nose }\end{array}$ & 1 & 2 & 3 & 4 & 5 \\
\hline $\begin{array}{l}\text { Coughing food or liquid out of } \\
\text { your mouth when it gets stuck }\end{array}$ & 1 & 2 & 3 & 4 & 5 \\
\hline
\end{tabular}

4. Next, please answer a few questions about how your swallowing problem has affected your diet and eating in the last month.

(circle one number on each line)

\begin{tabular}{|l|c|c|c|c|c|}
\hline & $\begin{array}{c}\text { Strongly } \\
\text { agree }\end{array}$ & Agree & Uncertain & Disagree & $\begin{array}{c}\text { Strongly } \\
\text { disagree }\end{array}$ \\
\hline $\begin{array}{l}\text { Figuring out what I can and can't } \\
\text { eat is a problem for me. }\end{array}$ & 1 & 2 & 3 & 4 & 5 \\
\hline $\begin{array}{l}\text { It is difficult to find foods that I } \\
\text { both like and can eat. }\end{array}$ & 1 & 2 & 3 & 4 & 5 \\
\hline
\end{tabular}




\section{Appendix I: Time Swallow Test}

Test Date:

Participant Code:

Timed Test of Swallowing Capacity for Neurological Patients

Pre-Test:

- Give the participant a tablespoon (approximately $10 \mathrm{ml}$ ) to drink. If they swallow without overt signs of coughing or choking proceed with the test.

Timed Swallow Test:

- Give the participant $150 \mathrm{ml}$ of cold tap water to drink from a standard glass.

- The instructions are to drink the water as quickly as possible when I tell you to begin.

- Once the examiner says go, time how long it takes to finish all the water and how many swallows were completed.

- If there is residual water in the cup then measure how much to determine the amount of water that was swallowed.

- Calculation:

○ $\underline{\text { Results }}=$

$$
\begin{aligned}
& \text { Avg Volume = } \\
& \text { (total ml/\# of swallows) }
\end{aligned}
$$

Avg Speed $=$ (total ml/seconds)

- Interpretation

- Swallowing speed $<10 \mathrm{ml} /$ second is a sensitive indicator of swallowing problems in neurological patients (Nathadwarawala, Nicklin, \& Wiles (1992). A timed test of swallowing capacity for neurological patients. Journal of Neurology, Neurosurgery, and Psychiatry, 55, 822-825.)

Clinician: 


\section{APPENDIX J: Past Medical History \\ INTERVIEW \\ Longitudinal Study of Communication, Nutrition and Physical Activity \\ Leslie Mahler, PhD, CCC-SLP, Principal Investigator \\ Ingrid Lofgren, $\mathrm{PhD}$, co-Investigator \\ Matthew Delmonico, PhD, co-Investigator \\ Version 1: 7-3-13}

Participant Name:

Initials:

ID\#:

Name of Interviewer:

Date:

Emergency contact name and address \& phone:

DIRECTIONS: Read the following questions out loud to each prospective volunteer and record the answers. Any answers that require clarification should be written in the space below the question or on the back of the sheet. Indicate whether any follow-up is necessary or if any referrals are appropriate.

What is your neurological diagnosis?

When were you diagnosed?

What were your symptoms at that

time?

What are your symptoms now? 
What is your communication

like?

What is your diet like?

What is your physical activity like?

Does your speech sound clear to other people?

If not, how does it sound?

Can you think of the words that you want to say?

If you do have trouble, how often does it happen?

Are you experiencing any symptoms of a swallowing disorder?

If yes, what is the problem with your swallowing?

What would you say is your most significant problem with speech or swallowing today? 
Did you experience any changes in your speech or swallowing before your diagnosis?

Does medication affect your speech or voice or swallowing? If yes, in what way?

\section{Speech}

How many hours of speaking do you do in a day?

What is a typical day of communicating like for you?

Do you pronounce your words clearly?

Do people ask you to repeat yourself?

Do people have a hard time understanding you?

What do you do when you want to be as easy to understand as possible?

What percent of your speech do you think is understandable? 
Has your neurological diagnosis caused you to talk less?

If so, how much less?

Why?

\section{Swallowing}

Do you have any difficulty with swallowing?

Do you cough during mealtimes?

If yes, do you cough more with water or solid food?

Do you have difficulty making the food go down (need to swallow twice)?

Does it take you longer to finish a meal than before your neurological diagnosis?

Have you experienced any unintentional recent weight loss?

Have you ever been diagnosed with pneumonia? If yes, when?

Have you changed your diet since your neurological diagnosis?

If yes, what did you modify?

\section{Musculoskeletal system:}

Has your doctor ever told you that you have: (circle all that apply)

- Osteoarthritis or degenerative arthritis

- Rheumatoid arthritis

- Osteoporosis 
- Ankylosing Spondylitis

- Unknown or other type of arthritis

- Any other disease of joint or muscle:

- Comments:

\section{Cardiovascular system:}

- Has any family member had a heart attack prior to the age of 55?

- If so, how are they related to you?

- Have you ever had frequent cramping in your legs while resting?

- If yes, is it a current problem?

- Have you ever had pain or cramping in your legs while walking?

If yes, is it a current problem?

- If yes, is this pain relieved by rest or by discontinuing walking?

- Have you ever been told that you have high blood pressure

If yes, what was the date of onset?

- Were you given any medications?

- Did a doctor ever tell you that you had a heart problem?

If yes: What was the date of onset?

- What did the doctor call it?

- Were you given any medications?

- Do you have any history of high cholesterol in your blood as evidenced by a previous blood lipid tests?

Comments:

\section{Endocrine system:}

Have you ever had any of the following: Thyroid problems, adrenal problems, diabetes mellitus? 
$-$

- If yes to diabetes, which type? Type 1 or Type 2

Date of onset-

Are/were you on any medication, or is it diet controlled?

\section{Neurological system:}

- Do you have any significant problems with your memory? (circle all that apply)

- When answering the telephone, do you recall what you were doing before it rang?

- Can you give the directions to your house/apartment?

- Can you keep appointments without a reminder?

- Can you remember what clothes you wore yesterday?

- Any problems with vision other than corrective lens changes?

- If yes, which of the following conditions- Blindness, temporary loss of vision, double vision, glaucoma, cataract, macular degeneration or others.

Do you have and of the following?: (circle all that apply)

- Vertigo (a feeling of spinning, or unsteadiness)

- Seizure or convulsions?

- Migraine or severe headaches?

- Paralysis of arm or leg?

- A head injury with loss of consciousness?

- Pain, numbness or tingling in your limbs?

- Pain in your lower back? 
- Do you have pain in any part of body including headaches while exercising?

- Have you been told that you have a peripheral neuropathy?

- Tremors?

- Problems with walking? If yes,

$\circ$ Do you fall frequently?

- Is your walking problem related to pain, weakness or loss of balance?

- Have you ever had an operation on skull or brain?

- Have you ever had meningitis or Brain fever?

Comments:

\section{Previous Treatment}

Have you had previous speech or swallow treatment, occupational therapy or physical therapy?

If yes, please describe (when, what)

Was it beneficial?

If yes, what changes did you notice? 


\section{Employment}

Are you employed?

Type of employment

How much speaking do you do at your job?

Other

Have you noticed any difficulty with your memory?

Have you experienced any changes in your mood?

Is it difficult for you to pay attention long enough to finish a task?

Do you have any difficulty reading?

Do you have any difficulty writing?

Do you have any other health problems or conditions that would affect communication, nutrition or physical activity? 


\section{APPENDIX K: Recruitment Materials}

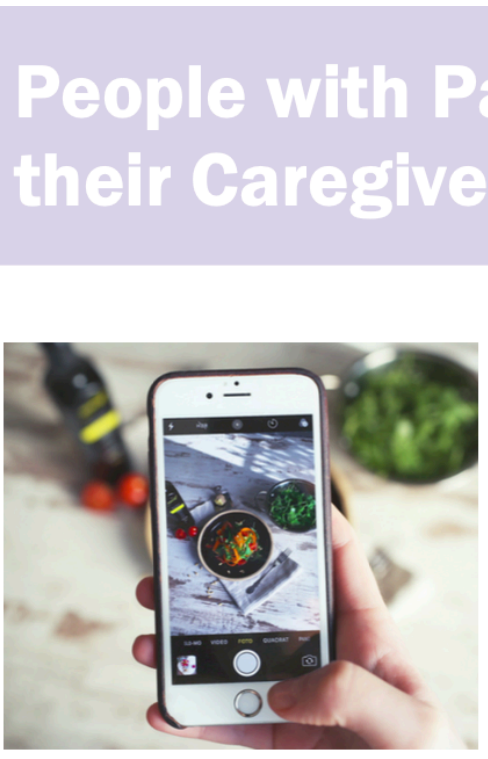

The University of Rhode Island's Lipid Lab invites people with Parkinson's disease and their caregivers to participate in a 4-part research study ( $\sim 4.5$ hours total) to collect information related to diet, access to health information and technology use. Dr. Ingrid Lofgren is the principal investigator of this URI research study, which has been approved by the URI Institutional Review Board.

\section{Inclusion Criteria:}

- $18^{+}$years of age

- Community dwelling

- English speaking

- Telephone access

- Participation of person with Parkinson's and their informal caregiver

- Informal caregiver is an unpaid friend/family member that provides majority of care

- No prior technology use or transportation access is required
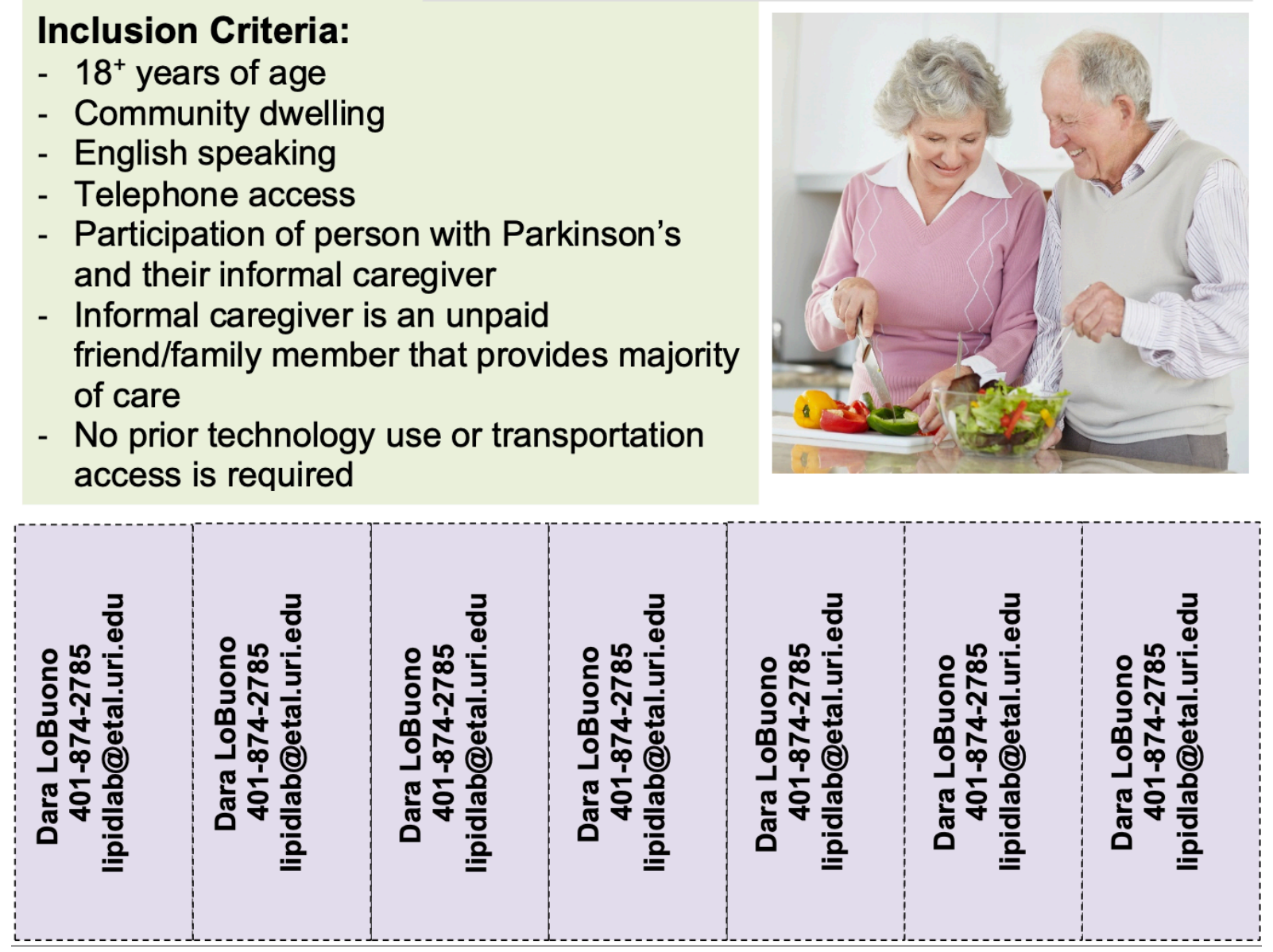
Dear

The University of Rhode Island's Lipid Lab is looking for people with Parkinson's disease and their caregivers (18 years + ) to participate in a research study to collect information related to diet, access to health information and technology use. The total time commitment for the study is approximately 4.5 hours and is completed over 4 time points.

Dr. Ingrid Lofgren, Department of Nutrition and Food Sciences, is the principal investigator for this study. We are asking you to display our recruitment flyer in your office, meeting area and/or lobby to help us recruit participants for this study. The flyer is attached to this email. If you know of any participants who are interested, please direct them to our recruitment flyer with our contact information.

Thank you for your time and support. If you have any questions regarding the study

please feel free to contact us.

Sincerely, Dara LoBuono MS RD LDN PhD Student Department of Nutrition and Food Sciences University of Rhode Island 401-874-2785 dara_lobuono@my.uri.edu 


\section{APPENDIX L: Parkinson's and Telenutrition Consent Form for Person with Parkinson's Disease}

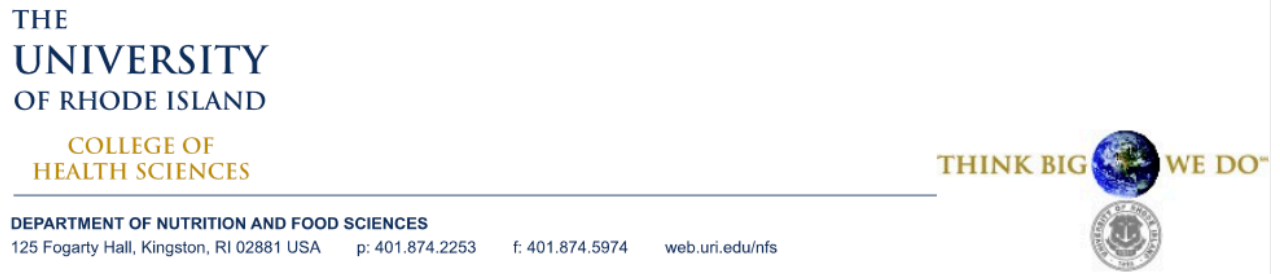

Tailoring Digital Health to Improve the Nutrition and Health of People with Parkinson's Disease Consent form for Person with Parkinson's Disease

Version 1: June 15, 2018

Principal Investigator, Ingrid E. Lofgren Co-Investigator, Leslie A. Mahler, PhD
401-874-5706

401-874-2490 ingridlofgren@uri.edu

Imahler@uri.edu

\section{Key Information}

- The purpose of this research project is to learn more about people with Parkinson's disease and their informal caregivers' perceptions of technology to manage health and food intake. Additionally, we want to better understand your food intake and current level of technology use.

- If you choose to participate you will be asked to complete 4 assessments total. Which include, an informational phone call, an in-person evaluation and 2-phone calls to collect 24-hour dietary recall information. The anticipated total study time is 4 hours and 20 minutes.

- There are minimal risks for participating in this study.

- Both you and your caregiver will receive personal health information, such as tailored dietary guidelines and body composition.

- You and your caregiver will be provided a copy of this consent form.

- Taking part in this research project is voluntary. You don't have to participate and you can stop it any time.

\section{Invitation}

You have been invited to take part in a research project described below. The purpose of the consent form is to provide you with details about the research study and to inform you of your rights if you agree to participate in the study. Your participation is voluntary. The researcher will explain the project to you in detail. You should feel free to ask questions. If you have more questions later you can call, Dr. Ingrid Lofgren, the study's Principal Investigator, at 401-8745706.

Why you are being asked to be in this research study?

To participate in this study, you must be at least 18 years old and speak English. You must also have a diagnosis of Parkinson's disease and an identified informal caregiver who will enroll in the study with you. Participation of both you and your caregiver is required.

With your permission, we will request the following health information from your physician:

- Date of diagnosis

- Current medications and treatments 
- Stage of Parkinson disease

You will sign a separate form to indicate whether you give your permission to release this health information for the study.

What is the reason for doing this research study?

The purpose of this research project is to learn more about your perceptions of technology using technology to manage heath. Additionally, we want to better understand your food intake and current level of technology use.

What will be done during this research study?

This study will be completed in four sessions, which will all occur within one month. The first session was the informational phone call you completed and cognitive screening. The Second Session will take place in person, either at one of the University of Rhode Island Speech and Hearing Clinic locations (Kingston or Pawtucket) or you will be offered the option of a home visit. The other 2 sessions will be completed by phone. The sessions are described below in detail and total participation time will be about 4 hours and 20 minutes. If there are any questions you do not wish to answer just let me know and we can skip it. You may also stop the interview/assessments at anytime. Our conversation and your responses will remain completely confidential.

Session 1 (Informational Phone Call, 15 minutes)

- This is the phone call you recently completed to learn about the study and screen for eligibility.

- The Telephone Montreal Cognitive Assessment (T-MoCA) was used to screen for eligibility. If you chose to enroll in the study, your T-MoCA results will be used for research purposes for this study only. If you do not choose to enroll in the study, T-MoCA information will be destroyed.

Session 2 ( $\sim 125$ minutes)

- This session will be completed in person today.

- You and your caregiver will complete a semi-structured interview designed by study staff. The interview will last about an hour and questions will revolve around Parkinson's and diet, access to health information and technology use. This interview will occur in a private space and will be audio recorded with your permission.

- During this time you will complete questionnaires related to dietary intake, swallowing, disease-burden, technology use, past medical history and demographics.

- A timed swallow test will be administered by a trained professional from Communicative Disorders.

- You will also have your height, weight, percent body fat and waist circumference measured.

Session 3 ( $\sim 60$ minutes total, $\sim 30$ minutes for person with Parkinson's disease)

- This session will be completed by phone. The study staff administering the phone call will be in a private room and have the phone on speaker. The call will be recorded with your permission.

- Both you and your caregiver will each complete a 24-hour dietary recall, which take each of you about 30 minutes to complete. A 24-hour dietary recall is a way to characterize your nutritional intake and consists of 5-steps to collect all the foods and beverages you consume in the past 24-hours. You will receive a Foods Amounts Booklet during Session 2

\begin{tabular}{|c|c|c|}
\hline & IRB NUMBER: & HU1819-001 \\
\hline $\begin{array}{l}\text { of RHODE B } \\
\text { nes }\end{array}$ & IRB APPROVAL DATE: & July 21,2018 \\
\hline
\end{tabular}


to help you estimate portion sizes for this part of the study. Along with dietary intake, we will also ask you questions about medication timing and supplement use.

Session 4 ( $\sim 60$ minutes total, $\sim 30$ minutes per person with Parkinson's disease)

- This session will be completed by phone. The study staff administering the phone call will be in a private room and have the phone on speaker. The call will be recorded with your permission.

- This will be the second dietary 24-hour recall that you complete and will take about 30 minutes.

What are the possible risks and benefits of being in this research study?

There are minimal risks for participating in this study. There may be some unknown or unanticipated risks, but every precaution will be taken to ensure your personal safety. You are being asked to participate in this study because we want to determine the best medium of digital health to help manage diet and increase access to nutrition and diet information. Understanding your preferences and your caregiver's preferences, will allow us to develop a tailored, userfriendly digital health nutrition intervention. Obtaining this information will also help use innovative mediums to increase access to nutrition information for managing Parkinson's disease. Both you and your caregiver will receive personal health information, such as tailored dietary guidelines and body composition. You will also receive information related to your thinking and language skills.

How will information about you be protected?

Reasonable steps will be taken to protect your privacy and the confidentiality of your study data. All information will be kept in research records that identify you private to the extent allowed by law. You will be assigned a participant number that will be kept with your response from questionnaires, interview, dietary data, medical history and demographics. The code number will be used on all response forms and in the analysis of the data. Results of this study may be used for purposes of research, educational lectures, and/or professional present. In publications you will be identified as "participant number." Dr. Lofgren and her research team will have sole access to all contact and personal information containing your name. This information will be kept in a locked filing cabinet in a locked laboratory. All electronic data will be stored through a secure server and will only be seen by the research team. All research data will be retained for a minimum of three years following completion of the study and then will be destroyed. Research data will be located in a locked filing cabinet in the principal investigator's laboratory.

The only persons who will have access to your research records are the study personnel, the Institutional Review Board (IRB), and any other person, agency, or sponsor as required by law. The information from this study may be published in scientific journals or presented at scientific meetings but the data will be reported as group or summarized data and your identity will be kept strictly confidential.

\section{Voluntary Participation}

Research studies include only people who agree to take part in the study. You can tell us you do not want to take part in the study. If you decide to take part in this study, you should understand that the evaluations are investigational and you may not experience any benefit from participation. The consent form will help make sure you understand the tasks included in the 
study before you decide whether you want to take part in the study. You may also quit the study at any time. This will not affect your relationship with the investigator or any groups you may be involved in at the University of Rhode Island or related to Parkinson's disease.

Costs and Compensation to Participants

You will not receive any compensation for your time in this study. If you chose to have your assessment at the University of Rhode Island, free parking will be provided.

What should you do if you have a problem during this research study?

Your welfare is the major concern of every member of the research team. If you have a problem as a direct result of being in this study, you should immediately contact one of the people listed at the beginning of this consent form.

What will happen if you decide not to be in this research study or decide to stop participating once you start?

You can decide not to be in this research study, or you can stop being in this research study ("withdraw') at any time before, during, or after the research begins for any reason. Deciding not to be in this research study or deciding to withdraw will not affect your relationship with the investigator or with the University of Rhode Island (list others as applicable).

What are your rights as a research subject? (rights and complaints):

You may ask any questions concerning this research and have those questions answered before agreeing to participate in or during the study. If you have any additional questions about this study, please feel free to contact Dr. Ingrid Lofgren at 401-874-5706. If you are not satisfied with the way this study is performed, you may discuss your complaints with Dr. Ingrid Lofgren (ingridlofgren@uri.edu, 401-874-5706), or Dr. Leslie Mahler (Imahler@uri.edu; 401-874-2490). You may also contact the Institutional Review Board at 401-874-4328/

researchintegrity@etal.uri.edu or the Vice President for Research and Economic Development for concerns or any questions about your rights as a research subject at: 70 Lower College Road, University of Rhode Island, Kingston, RI at (401) 874-4328 and speak to them anonymously if you choose.

\section{Documentation of Informed Consent}

You are voluntarily making a decision whether or not to be in this research study. Signing this form means that (1) you have read and understood this consent form, (2) you have had the consent form explained to you, (3) you have had your questions answered and (4) you have decided to be in the research study. You will be given a copy of this consent form to keep.

Signature of Participant

$\overline{\text { Participant Typed/printed Name }}$

Date
Signature of Researcher

Researcher Typed/printed name

Please keep a copy of the signed consent form for your records. 


\section{AUDIO/VIDEO ADDENDUM TO THE CONSENT FORM FOR RESEARCH}

By signing this consent form, I confirm that I give my permission for audio recording(s) of me, to be used for the purposes listed above, and to be retained for three years after study completion. You may still participate in this study if you are not willing to be recorded.

Signature of Participant

Participant Typed/printed Name

Date
Signature of Researcher

Researcher Typed/printed name

ADDENDUM FOR HIPAA INFORMATION (PERSONAL HEALTH INFORMATION) ACCESS You have rights regarding the privacy of your medical information collected before and during this research. This medical information, called "protected health information" (PHI), typically may include, depending upon the nature of this research, demographic information (like your address and birth date), the results of physical exams, blood tests, $x$-rays and other diagnostic and medical procedures, as well as your medical history.

By signing this consent form, you are allowing the research team to have access to your PHI. The research team includes the investigators listed on this consent form and other personnel involved in this specific study at [add additional personnel/institutions as applicable].

Your PHI will be used only for the purpose(s) described in the section "What is the reason for doing this research study?"

Your PHI will be shared, as necessary, with the Institutional Review Board (IRB) and with any person or agency required by law. You are also allowing the research team to share your PHI with other people or groups listed below all of these persons or groups listed below are obligated to protect your PHI.

The protected health information that may be used and disclosed includes:

- Date of Parkinson's Diagnosis

- Medication dosing and timing for Parkinson's

- Treatments for Parkinson's disease, such as Deep Brain Stimulation

- Stage of Parkinson's as measured by Hoehn \& Yahr and Unified Parkinson's Disease Rating Scale

You may cancel your authorization for further collection of $\mathrm{PHI}$ for use in this research at any time by contacting the principal investigator in writing. However, the PHI which is not included in the 
research data obtained to date may still be used. If you cancel this authorization, you will no longer be able to participate in this research.

You are authorizing us to use and disclose your $\mathrm{PHI}$ for as long as the research study is being conducted.

Signature of Participant

$\overline{\text { Participant Typed/printed Name }}$

Date
Signature of Researcher

$\overline{\text { Researcher Typed/printed name }}$

Date 


\title{
APPENDIX M: Parkinson's and Telenutrition Consent Form for Caregiver
}

\author{
THE \\ UNIVERSITY \\ OF RHODE ISLAND \\ COLLEGE OF \\ HEALTH SCIENCES \\ THINKBIG WE DO \\ DEPARTMENT OF NUTRITION AND FOOD SCIENCES \\ 125 Fogarty Hall, Kingston, RI 02881 USA $\begin{array}{lll}\text { p: } 401.874 .2253 & \text { f. } 401.874 .5974 & \text { web.uri.edu/nfs }\end{array}$
}

Tailoring Digital Health to Improve the Nutrition and Health of People with Parkinson's Disease Consent form for Caregivers

Version 1: June 15, 2018

Principal Investigator, Ingrid E. Lofgren

401-874-5706

ingridlofgren@uri.edu

Co-Investigator, Leslie A. Mahler, PhD

401-874-2490

Imahler@uri.edu

\section{Key Information}

- The purpose of this research project is to learn more about people with Parkinson's disease and their informal caregivers' perceptions of technology to manage health and food intake. Additionally, we want to better understand your food intake and current level of technology use.

- If you choose to participate you will be asked to complete 4 assessments total. Which include, an informational phone call, an in-person evaluation and 2-phone calls to collect 24-hour dietary recall information. The anticipated total study time is 4 hours and 20 minutes.

- There are minimal risks for participating in this study.

- Both you and your loved one with Parkinson's disease will receive personal health information, such as tailored dietary guidelines and body composition.

- You and your loved one will be provided a copy of this consent form.

- Taking part in this research project is voluntary. You don't have to participate, and you can stop it any time.

\section{Invitation}

You have been invited to take part in a research project described below. The purpose of the consent form is to provide you with details about the research study and to inform you of your rights if you agree to participate in the study. Your participation is voluntary. The researcher will explain the project to you in detail. You should feel free to ask questions. If you have more questions later you can call, Dr. Ingrid Lofgren, the study's Principal Investigator, at 401-8745706.

Why you are being asked to be in this research study?

To participate in this study, you must be at least 18 years old and speak English. You must be an informal caregiver for someone with Parkinson's disease. Your loved one with Parkinson's disease must also enroll in the study in order for you to participate. An informal caregiver is defined as: an unpaid family member or friend who provides the majority of care for their loved one with Parkinson's disease.

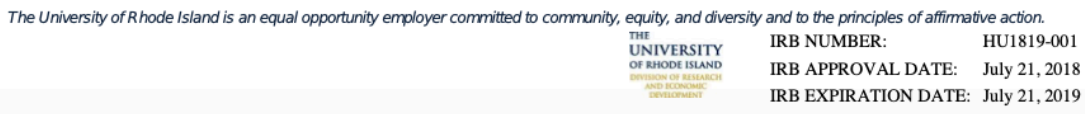


What is the reason for doing this research study?

The purpose of this research project is to learn more about your perceptions of technology using technology to manage heath. Additionally, we want to better understand your food intake and current level of technology use.

What will be done during this research study?

This study will be completed in four sessions, which will all occur within one month. The first session was the informational phone call you completed and cognitive screening. The Second Session will take place in person, either at one of the University of Rhode Island Speech and Hearing Clinic locations (Kingston or Pawtucket) or you will be offered the option of a home visit. The other 2 sessions will be completed by phone. The sessions are described below in detail and total participation time will be about 4 hours and 20 minutes. If there are any questions you do not wish to answer just let me know and we can skip it. You may also stop the interview/assessments at anytime. Our conversation and your responses will remain completely confidential.

Session 1 (Informational Phone Call, 15 minutes)

- This is the phone call you recently completed to learn about the study and screen for eligibility.

- The Telephone Montreal Cognitive Assessment (T-MoCA) was used to screen for eligibility. If you chose to enroll in the study, your T-MoCA results will be used for research purposes for this study only. If you do not chose to enroll in the study, T-MoCA information will be destroyed.

Session 2 ( 125 minutes)

- This session will be completed in person today.

- You and your loved one with Parkinson's disease will complete a semi-structured interview designed by study staff. The interview will last about an hour and questions will revolve around Parkinson's and diet, access to health information and technology use. This interview will occur in a private space and will be audio recorded with your permission.

- During this time, you will complete questionnaires related to dietary intake, swallowing, disease-burden, technology use, past medical history and demographics.

- A timed swallow test will be administered by a trained professional from Communicative Disorders.

- You will also have your height, weight, percent body fat and waist circumference measured.

Session 3 ( $\sim 60$ minutes total, $\sim 30$ minutes for person with Parkinson's disease)

- This session will be completed by phone. The study staff administering the phone call will be in a private room and have the phone on speaker. The call will be recorded with your permission.

- Both you and loved one with Parkinson's disease will each complete a 24-hour dietary recall, which take each of you about 30 minutes to complete. A 24-hour dietary recall is a way to characterize your nutritional intake and consists of 5-steps to collect all the foods and beverages you consume in the past 24-hours. You will receive a Foods Amounts Booklet during Session 2 to help you estimate portion sizes for this part of the study. Along with dietary intake, we will also ask you questions about medication timing and supplement use. 
Session 4 ( $\sim 60$ minutes total, $\sim 30$ minutes per person)

- This session will be completed by phone. The study staff administering the phone call will be in a private room and have the phone on speaker. The call will be recorded with your permission.

- This will be the second dietary 24-hour recall that you complete and will take about 30 minutes.

- You may also participate in your loved one with Parkinson's disease's 24-hour dietary recall.

What are the possible risks and benefits of being in this research study?

There are minimal risks for participating in this study. There may be some unknown or unanticipated risks, but every precaution will be taken to ensure your personal safety. You are being asked to participate in this study because we want to determine the best medium of digital health to help manage diet and increase access to nutrition and diet information. Understanding your preferences, will allow us to develop a tailored, user-friendly digital health nutrition intervention. Obtaining this information will also help use innovative mediums to increase access to nutrition information for managing Parkinson's disease. Both you and your loved one with Parkinson's disease will receive personal health information, such as tailored dietary guidelines and body composition. You will also receive information related to your thinking and language skills.

\section{How will information about you be protected?}

Reasonable steps will be taken to protect your privacy and the confidentiality of your study data. All information will be kept in research records that identify you private to the extent allowed by law. You will be assigned a participant number that will be kept with your response from questionnaires, interview, dietary data, medical history and demographics. The code number will be used on all response forms and in the analysis of the data. Results of this study may be used for purposes of research, educational lectures, and/or professional present. In publications you will be identified as "participant number." Dr. Lofgren and her research team will have sole access to all contact and personal information containing your name. This information will be kept in a locked filing cabinet in a locked laboratory. All electronic data will be stored through a secure server and will only be seen by the research team. All research data will be retained for a minimum of three years following completion of the study and then will be destroyed. Research data will be located in a locked filing cabinet in the principal investigator's laboratory.

The only persons who will have access to your research records are the study personnel, the Institutional Review Board (IRB), and any other person, agency, or sponsor as required by law. The information from this study may be published in scientific journals or presented at scientific meetings but the data will be reported as group or summarized data and your identity will be kept strictly confidential.

\section{Voluntary Participation}

Research studies include only people who agree to take part in the study. You can tell us you do not want to take part in the study. If you decide to take part in this study, you should understand that the evaluations are investigational, and you may not experience any benefit from participation. The consent form will help make sure you understand the tasks included in the study before you decide whether you want to take part in the study. You may also quit the study 
at any time. This will not affect your relationship with the investigator or any groups you may be involved in at the University of Rhode Island or related to Parkinson's disease.

\section{Costs and Compensation to Participants}

You will not receive any compensation for your time in this study. If you chose to have your assessment at the University of Rhode Island, free parking will be provided.

What should you do if you have a problem during this research study?

Your welfare is the major concern of every member of the research team. If you have a problem as a direct result of being in this study, you should immediately contact one of the people listed at the beginning of this consent form.

\section{What will happen if you decide not to be in this research study or decide to stop} participating once you start?

You can decide not to be in this research study, or you can stop being in this research study ("withdraw') at any time before, during, or after the research begins for any reason. Deciding not to be in this research study or deciding to withdraw will not affect your relationship with the investigator or with the University of Rhode Island (list others as applicable).

What are your rights as a research subject? (rights and complaints):

You may ask any questions concerning this research and have those questions answered before agreeing to participate in or during the study. If you have any additional questions about this study, please feel free to contact Dr. Ingrid Lofgren at 401-874-5706. If you are not satisfied with the way this study is performed, you may discuss your complaints with Dr. Ingrid Lofgren (ingridlofgren@uri.edu, 401-874-5706), or Dr. Leslie Mahler (Imahler@uri.edu; 401-874-2490). You may also contact the Institutional Review Board at 401-874-4328/

researchintegrity@etal.uri.edu or the Vice President for Research and Economic Development for concerns or any questions about your rights as a research subject at: 70 Lower College Road, University of Rhode Island, Kingston, RI at (401) 874-4328 and speak to them anonymously if you choose.

\section{Documentation of Informed Consent}

You are voluntarily making a decision whether or not to be in this research study. Signing this form means that (1) you have read and understood this consent form, (2) you have had the consent form explained to you, (3) you have had your questions answered and (4) you have decided to be in the research study. You will be given a copy of this consent form to keep.

Signature of Participant

Participant Typed/printed Name

Date
Signature of Researcher

$\overline{\text { Researcher Typed/printed name }}$

Please keep a copy of the signed consent form for your records.

\begin{tabular}{|c|c|c|}
\hline & IRB NUMBER: & HU1819-001 \\
\hline 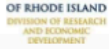 & $\begin{array}{l}\text { IRB APPROVAL DATE: } \\
\text { IRB EXPIRATION DATE: }\end{array}$ & $\begin{array}{l}\text { July } 21,2018 \\
\text { Jul } 21,2019\end{array}$ \\
\hline
\end{tabular}




\section{AUDIO/VIDEO ADDENDUM TO THE CONSENT FORM FOR RESEARCH}

By signing this consent form, I confirm that I give my permission for audio recording(s) of me, to be used for the purposes listed above, and to be retained for three years after study completion. You may still participate in this study if you are not willing to be recorded.

Signature of Participant

$\overline{\text { Participant Typed/printed Name }}$

Date
Signature of Researcher

$\overline{\text { Researcher Typed/printed name }}$
Date

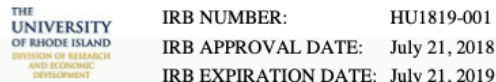


APPENDX N: Moderator Guide for Semi-Structured Dyadic Interviews

\section{Moderator Guide and Script}

Script: Hello my name is Dara LoBuono. I am a PhD student at the University of Rhode Island in the Department of Nutrition and Food Sciences. Today we will discuss how you get health information, how you would like to get health information and what would help you get this information more easily. Remember, there are no right or wrong answers, so please answer questions as best as you can. As a reminder, I will be recording our discussion and taking notes for research purposes. Before we begin, do you have any questions?

Transition: Great! Let's get started. The first few questions will be about Parkinson's disease and what you eat.

\section{$P D$ and Diet}

1. Do you make different food choices as a result of having PD? Please explain.

a. Probes: Sugar intake? Fluid Intake? Types of food you eat?

b. Prompt: In what ways is eating more difficult for you as a result of Parkinson's disease?

Follow up: How does Parkinson's disease impact the amount you eat?

c. Probes: Changes due to: Taste and smell? Constipation? Appetite? Time it takes to cook a meal or limited time to cook meals?

2. What food choices or diet modifications help you manage the symptoms of your Parkinson's disease? Why or why not?

a. Probes: Changing textures to minimize difficulty chewing and swallowing? Eating certain foods to manage constipation?

Transition: Now we will discuss your ability to get nutrition and health information and ways that may help you increase access to these services.

\section{Accessibility of Nutrition and Health Information}

3. How does having Parkinson's disease influence your ability to get health information? (Are you able to find the health information you need for managing Parkinson's?)

4. How do you access health information for managing Parkinson's disease?

5. Where do you get information about foods and diets for people with Parkinson's disease?

a. Follow up: Please describe what information was provided. 
6. What information related to eating do you want to learn more about? Please explain.

7. How would you like to receive information about food and eating?

a. Probes: At visits with a healthcare provider, pamphlets, the internet, support groups

b. Follow up: How often?

Transition: Thank you for that information. Now we are going to explore information related technology* for managing Parkinson's disease. For the purposes of this interview, technology will be defined as: tools that support independent living, social interaction and assist in managing and providing care. Examples include computers and internet. When responding to these questions, please think about forms of technology that either of you may use or be interested in using.

\section{Digital Health for PD:}

8. What forms of technology do you currently use and for what purpose?

a. Probes: computers, tablets, internet, e-mail, smart phones*, smart-watches* *Smart phones - a cell phone that also includes internet access, camera, email access, and storage. (Example: iPhone, Pixel, Android)

* Smart watches - a wristwatch that can also perform tasks similar to a smart phone or computer. (Example: Apple Watch)

9. What does the term digital health* mean to you?

Transition: So, for the purpose of this study, digital health is a broad term used to describe technologies that better manage and track health.

10. What digital health products do you currently use?

a. Probes: Apps* for medication management, devices to monitor gait/speech/blood pressure, videoconferencing*

*Apps - also known as mobile or computer applications are programs designed to perform a function, task or activity that can benefit the user

*Videoconferencing - is a technology that allows two or more people to communicate from different locations through video and audio signals. Like talking on the phone, but you can see the person (Example: Facetime, Skype)

11. What (additional) digital health products would you be willing to try and why? a. Probes: Apps, devices to monitor gait/speech, * wearables*, 
videoconferencing, websites

i. Wearable device to Monitor Gait/Speech $\rightarrow$ sensors (ankle bracelet or sock) that measure your stride length and time. Information collected can determine if you are shuffling or taking short steps and can provide information about disease progression.

ii. wearables such as fit bits and apple watches are devices that can track steps taken, heart rate, calories burned and sleep quality. There is also one that helps manage tremors and writing.

12. What digital health products would you be willing to try to help manage food and eating for Parkinson's disease and why?

13. The following scenarios are possible ways digital health can be used to make information Tell me if you would be interested in learning more about utilizing the following example of digital health and explain why.

a. Using videoconferencing to have nutrition counseling sessions from home.

b. Wearing a watch at meals and snacks that counts your bites*.

Bite Counter - a watch that tracks wrist motion to count bites and estimate calories while you eat. It provides real-time feedback on amount consumed.

c. Taking pictures of meals and snacks for a nutrition professional to review.

d. A website that allows you to post questions to nutrition professionals and other people living with Parkinson's disease and their caregivers about nutrition.

e. Using phone applications and websites to track the food you eat. (example: MyFitness Pal, Lose it,

14. What makes technology and digital health useful for you?

15. What makes it difficult for you to use digital health (more)?

16. What else should I know about how digital health could help you with your eating to optimize your management of Parkinson disease?

Transition: Thank you for answering all of my questions. We have a few more questions related to the information we discussed today. I will read you the question/statement and the possible responses. Please select one choice that most closely aligns with your response.

\section{Quantitative Questions.}

17. How important is knowledge about your disease to you? 
1. Unimportant 2. Somewhat Important 3. Neutral 4. Important 5. Very important.

\begin{tabular}{|c|c|c|c|c|}
\hline \multirow{2}{*}{$\begin{array}{c}\leftarrow \\
\text { Unimportant }\end{array}$} & & & & \\
\hline & Somewhat & Neutral & Important & Very \\
\hline & Important & & & Important \\
\hline
\end{tabular}

18. Which of these is your main source of knowledge about your disease? (Please Pick one).

1. Healthcare

2. Patients organizations

3. Found myself online

4. Other patients

5. Family, relatives, and friends

6. Other sources.

19. Have you been able to find the knowledge you need about your disease?

\begin{tabular}{|c|c|c|c|c|}
\hline 1 & 1 & 1 & 1 & 1 \\
\hline Not at all & Rarely & $\begin{array}{l}\text { Some of the } \\
\text { time }\end{array}$ & $\begin{array}{l}\text { Most of the } \\
\text { time }\end{array}$ & Absolutely \\
\hline
\end{tabular}

20. How strongly do you agree or disagree that it is important to follow an eating plan for managing PD?

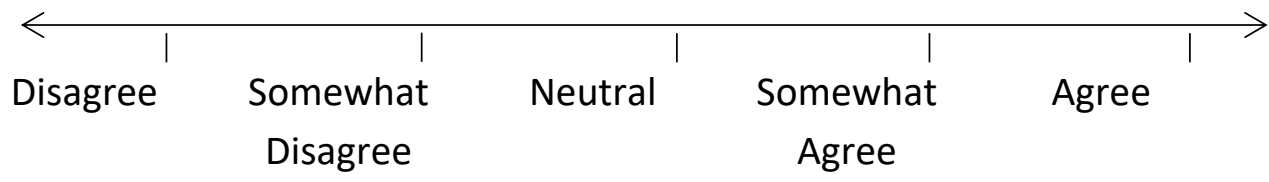

21. How easy or difficult is it to find information related to healthy eating for Parkinson's?

_ Very Difficult

Difficult

_ Somewhat Difficult

_Easy

_ Very Easy 
22. How helpful would it be to work with a nutrition professional to manage eating for Parkinson's disease?

Very Unhelpful

Unhelpful

Somewhat unhelpful

Neither unhelpful or helpful

Somewhat Helpful

Helpful

Very Helpful

23. Since being diagnosed with Parkinson's disease my appetite has:

\begin{tabular}{|c|c|c|c|c|}
\hline 1 & | & 1 & | & 7 \\
\hline Decreased & Slightly & Stayed the & Slightly & Increased \\
\hline
\end{tabular}

24. Since being diagnosed with Parkinson's disease the overall amount you eat each day has:

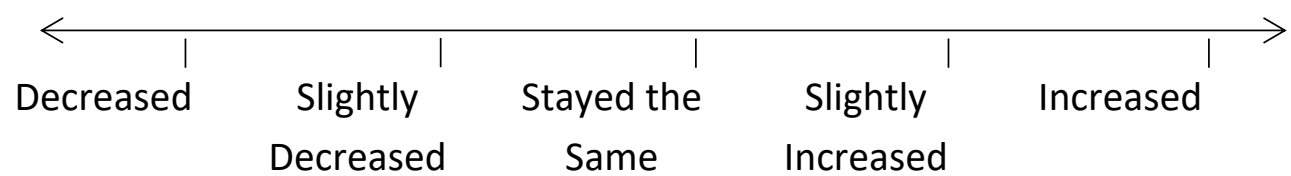

Script: This concludes the interview. Thank you for all your valuable information and participation in this study. 
APPENDIX O: Supporting Documents for 24-Hour Dietary Recall Data
Collected

\section{4-Hour Recall Questions}

Spring 2018

All enrolled participants will complete two 24-hour recalls. During Sessions 3 and 4 (via telephone), PwPD and caregivers will complete two 24-hour dietary recalls; detailed information about food and beverages intake from midnight to midnight the previous day. Each 24 -hour recall will last about 30 minutes per participant.

A $24 \mathrm{HR}$ is when a person is asked to list and describe all the foods they ate the previous day. Since people consume different foods and beverages, different questions are asked during each 24-hour. Participants will be encouraged to have labels of foods they eat available during the assessments so exact information can be entered. This project will be utilizing the Nutrition Data System for Research from the University of Minnesota to collect the dietary data. Information on the program is attached. For the most part, all participants will be asked the following questions. Many of these questions will be asked multiple times.

- At what time did you get up yesterday?

- What was the first thing you had to eat or drink after getting up yesterday?

- What else did you have to eat with that (insert food or beverage)?

- What was added to that beverage?

- What else did you have at that meal?

- Was the (insert food or beverage) eaten plain or did you put something on it?

- What did you eat after that meal?

- What did you have for snacks yesterday?

- What was the brand of (insert food or beverage)?

- How many cans/bottles or juice/soda/water did you have at that time?

- What was the last thing you ate and drank yesterday?

- What did you snack on after you last meal?

- Did you get up during the night and eat anything?

- About what size was the (insert food or beverage)?

- Was the (insert food or beverage) an original product or was it modified in anyway? For example, was it low sodium, low fat, cholesterol free, etc.?

- When was the first time you took your levodopa or levodopa containing medication?

- When was the next time you took your levodopa or levodopa containing medication? Did you have anything to eat at this time? 
Depending on dietary intake, these additional questions may be asked. Many of these questions could be asked multiple times.

- Did you add any cream, milk, milk substitute to the coffee or tea?

- Did you add any sugar or sugar substitute to the coffee or tea?

- How much of the (insert food or beverage) did you eat?

- Were you able to finish all of that (insert food or beverage)?

- Was this (insert food) prepared with fat? If so, what type of fat?

- When preparing (insert food) was salt added?

- Was there frosting on the (insert food)?

- If so, about how much frosting?

- What was the flavor/color of the frosting?

- Did you add any condiments to (insert food)?

- If so, what condiments and how much of each?

- Was there ice in the (insert beverage)?

- Was the cake a single, double, or triple layer cake?

- Were there any seeds on the bagel?

\section{Session 3: Phone Call Script}

“Good Morning/Afternoon/Evening

Today we will be discussing your dietary intake as part of the URI Parkinson's disease research

study. This is the third of four sessions. Both you and your loved one will each be completing a 24-hour recall today.

We will go over all of the foods and beverages that you consumed yesterday, from midnight to midnight. This phone call should last approximately 60 minutes or about 30 minutes per recall."

- Study staff will proceed to administer the 24-hour dietary recalls, one with the PwPD and one with the caregiver. Each recall is anticipated to be about 30 minutes.

"Thank you for your participation in this third assessment, I will be calling in the near future to complete your fourth and final session, another 24-hour dietary recall session by phone."

\section{Session 4: Phone Call Script}

"Good Morning/Afternoon/Evening

Today we will be discussing your dietary intake as part of the URI Parkinson's disease research 
study. This is the fourth and final session. Both you and your loved one will each be completing a 24-hour recall today.

We will go over all of the foods and beverages that you consumed yesterday, from midnight to midnight. This phone call should last approximately 60 minutes or about 30 minutes per recall."

- Study staff will proceed to administer the 24-hour dietary recalls, one with the PWPD and one with the caregiver. Each recall is anticipated to be about 30 minutes.

"Thank you for your participation in this final session. Both you and your loved one will be receiving individual dietary analyses and recommendations in the mail in the next few weeks" 


\section{APPENDIX P: EU Indicators of Digital Competence}

Tailoring Digital Health to Improve the Nutrition and Health of People with Parkinson's Disease

\section{Participant ID\#}

Date

Questions Adopted from the European Union Wide Indicators of Digital Competence The following questions relate to your technology use

1. ACTIVITIES: For the activities listed below, please select the answers that best reflects how often you take part in that activity:

\begin{tabular}{|l|c|c|c|c|}
\hline & Never & $\begin{array}{c}\text { Rarely } \\
\text { (less than } \\
\text { once a } \\
\text { month) }\end{array}$ & $\begin{array}{c}\text { Sometimes } \\
\text { (at least } \\
\text { once a } \\
\text { month) }\end{array}$ & $\begin{array}{c}\text { Often (at } \\
\text { least once } \\
\text { a week) }\end{array}$ \\
\hline Doing unpaid community service or volunteer work & $\square$ & $\square$ & $\square$ & $\square$ \\
\hline Doing paid community work & $\square$ & $\square$ & $\square$ & $\square$ \\
\hline Taking courses or participating in discussion groups & $\square$ & $\square$ & $\square$ & $\square$ \\
\hline Going to a movie, restaurant or sporting event & $\square$ & $\square$ & $\square$ & $\square$ \\
\hline Participating in social and community groups & $\square$ & $\square$ & $\square$ & $\square$ \\
\hline Talking on the phone & $\square$ & $\square$ & $\square$ & $\square$ \\
\hline Visiting friends & $\square$ & $\square$ & $\square$ & $\square$ \\
\hline Attending group exercise activities & $\square$ & $\square$ & $\square$ & $\square$ \\
\hline $\begin{array}{l}\text { Corresponding with friends and family on the } \\
\text { internet (Facebook, FaceTime, Skype) }\end{array}$ & $\square$ & $\square$ & $\square$ & $\square$ \\
\hline
\end{tabular}

\section{How frequently do you use the following technological devices?}

\begin{tabular}{|l|c|c|c|c|}
\hline & Daily & Weekly & Monthly & Never \\
\hline Desktop Computer & $\square$ & $\square$ & $\square$ & $\square$ \\
\hline Laptop Computer & $\square$ & $\square$ & $\square$ & $\square$ \\
\hline Tablet (e.g., iPad, Kindle) & $\square$ & $\square$ & $\square$ & $\square$ \\
\hline Smartphone (e.g., iPhone, Android) & $\square$ & $\square$ & $\square$ & $\square$ \\
\hline Other: & $\square$ & $\square$ & $\square$ & $\square$ \\
\hline
\end{tabular}

3. For what purpose(s) do you use technological devices? (Please select all that apply)
$\square$ Email
$\square$ Search the Internet
$\square$ Social Media (Facebook, Twitter)
$\square$ Online Banking or Paying Bills
$\square$ Watch Videos (YouTube)
$\square$ Shopping
$\square$ Video Conferencing (Skype, FaceTime
$\square$ Other (please specify):

or Google Hangout) 
Tailoring Digital Health to Improve the Nutrition and Health of People with Parkinson's Disease

4. How much do you agree with the statements, I feel competent:

\begin{tabular}{|l|c|c|c|c|}
\hline & $\begin{array}{c}\text { Strongly } \\
\text { Disagree }\end{array}$ & $\begin{array}{c}\text { Slightly } \\
\text { Disagree }\end{array}$ & $\begin{array}{c}\text { Slightly } \\
\text { Agree }\end{array}$ & $\begin{array}{c}\text { Strongly } \\
\text { Agree }\end{array}$ \\
\hline Searching and finding information about goods and services & $\square$ & $\square$ & $\square$ & $\square$ \\
\hline $\begin{array}{l}\text { Reading or downloading online news/newspapers/news } \\
\text { magazines }\end{array}$ & $\square$ & $\square$ & $\square$ & $\square$ \\
\hline Using copy/paste tools & $\square$ & $\square$ & $\square$ & $\square$ \\
\hline Seeking health information & $\square$ & $\square$ & $\square$ & $\square$ \\
\hline Sending/receiving emails & $\square$ & $\square$ & $\square$ & $\square$ \\
\hline Using videocalls, such as Skype & $\square$ & $\square$ & $\square$ & $\square$ \\
\hline Participating in social networks & $\square$ & $\square$ & $\square$ & $\square$ \\
\hline Posting messages on social networks & $\square$ & $\square$ & $\square$ & $\square$ \\
\hline Uploading self-created content to any website to be shared & $\square$ & $\square$ & $\square$ & $\square$ \\
\hline Sharing talents or interests on social networks & $\square$ & $\square$ & $\square$ & $\square$ \\
\hline Sharing my interests and ideas with those you know & $\square$ & $\square$ & $\square$ & $\square$ \\
\hline Connecting and installing new devices & $\square$ & $\square$ & $\square$ & $\square$ \\
\hline Internet banking & $\square$ & $\square$ & $\square$ & $\square$ \\
\hline $\begin{array}{l}\text { Buying or ordering goods or services for private use (last 12 } \\
\text { months) over the internet }\end{array}$ & $\square$ & $\square$ & $\square$ & $\square$ \\
\hline Making an appointment with a practitioner via a website & $\square$ & $\square$ & $\square$ & $\square$ \\
\hline
\end{tabular}


APENDIX Q: 2015: Health Information National Trends Survey (HINTS)

Tailoring Digital Health to Improve the Nutrition and Health of People with Parkinson's Disease

\section{Participant ID\#}

Date

Questions Adopted from Health Information National Trends Survey

The following questions are related to how you look for overall health information, eating and food, and/or Parkinson's disease.

1. Have you ever looked for information about health or medical topics from any source?

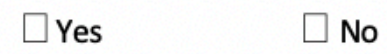

2. The most recent time you looked for information about health or medical topics, where did you go first? Mark only one.

$\begin{array}{ll}\square \text { Books } & \square \text { Internet } \\ \square \text { Brochures, pamphlets, etc. } & \square \text { Library } \\ \square \text { Public Health organization } & \square \text { Magazines } \\ \square \text { Family } & \square \text { Newspapers } \\ \square \text { Friend/Coworker } & \square \text { Telephone information number } \\ \square \text { Doctor or health care provider } & \square \text { Complementary, alternative, or } \\ & \text { unconventional practitioner }\end{array}$

3. Based on the results of your most recent search for information about health or medical topics, how much do you agree or disagree with each of the following statements?

\begin{tabular}{|l|c|c|c|c|}
\hline & $\begin{array}{c}\text { Strongly } \\
\text { Agree }\end{array}$ & $\begin{array}{c}\text { Somewhat } \\
\text { Agree }\end{array}$ & $\begin{array}{c}\text { Somewhat } \\
\text { Disagree }\end{array}$ & $\begin{array}{c}\text { Strongly } \\
\text { Disagree }\end{array}$ \\
\hline $\begin{array}{l}\text { a. It took a lot of effort to get the } \\
\text { information needed }\end{array}$ & $\square$ & $\square$ & $\square$ & $\square$ \\
\hline $\begin{array}{l}\text { b. You felt frustrated during a search } \\
\text { for information }\end{array}$ & $\square$ & $\square$ & $\square$ & $\square$ \\
\hline $\begin{array}{l}\text { c. You were concerned about the } \\
\text { quality of the information }\end{array}$ & $\square$ & $\square$ & $\square$ & $\square$ \\
\hline $\begin{array}{l}\text { d. The information you found was hard } \\
\text { to understand }\end{array}$ & $\square$ & $\square$ & $\square$ & $\square$ \\
\hline
\end{tabular}

4. Overall, how confident are you that you could get health information if you needed it? Circle one of the following choices:

\begin{tabular}{c|ccccc} 
(1) & (2) Very & (3) & (4) A little & (5) Not at all \\
completely & confident & Somewhat \\
confident & & $\begin{array}{c}\text { confident } \\
\text { confident }\end{array}$ &
\end{tabular}


5. In general, how much would you trust information about health or medical topics from each of the following?

\begin{tabular}{|l|c|c|c|c|}
\hline & Not at all & A little & Some & A lot \\
\hline a. A doctor & $\square$ & $\square$ & $\square$ & $\square$ \\
\hline b. A healthcare provider & $\square$ & $\square$ & $\square$ & $\square$ \\
\hline c. Family or friends & $\square$ & $\square$ & $\square$ & $\square$ \\
\hline $\begin{array}{l}\text { c. Government health agencies (e.g. the Food and Drug } \\
\text { Administration (FDA), National Institute of Health (NIH), or } \\
\text { Centers for Disease Control (CDC)) }\end{array}$ & $\square$ & $\square$ & $\square$ & $\square$ \\
\hline $\begin{array}{l}\text { d. Health organizations or groups (e.g. American } \\
\text { Parkinson's Disease Association (APDA)) }\end{array}$ & $\square$ & $\square$ & $\square$ & $\square$ \\
\hline e. Charitable organizations & $\square$ & $\square$ & $\square$ & $\square$ \\
\hline f. Religious organizations and leaders & $\square$ & $\square$ & $\square$ & $\square$ \\
\hline g. Online forums and blogs & $\square$ & $\square$ & $\square$ & $\square$ \\
\hline
\end{tabular}

6. On a typical weekday about how many hours do you...

\begin{tabular}{|l|l|}
\hline & Hours \\
\hline a. Watch television & \\
\hline b. Listen to the radio & \\
\hline c. Use the internet for personal reasons & \\
\hline
\end{tabular}

7. During a typical weekend, about how many hours do you...

\begin{tabular}{|l|c|}
\hline & Hours \\
\hline a. Watch television & \\
\hline b. Listen to the radio & \\
\hline c. Use the internet for personal reasons & \\
\hline
\end{tabular}

8. In the past seven days, how many days did you read a newspaper?<smiles>C1CCC1</smiles>

9. What information did you look for the most recent time you looked for information about Parkinson's disease?

10. What information did you look for the most recent time you looked for information about food and diet? 
Tailoring Digital Health to Improve the Nutrition and Health of People with Parkinson's Disease

11. In general, how much would you trust information about Parkinson's from each of the following?

\begin{tabular}{|l|c|c|c|c|}
\hline & Not at all & A little & Some & A lot \\
\hline a. A doctor/pharmacist/health care provider & $\square$ & $\square$ & $\square$ & $\square$ \\
\hline b. Family or friends & $\square$ & $\square$ & $\square$ & $\square$ \\
\hline c. Government health agencies (e.g. FDA, NIH, CDC) & $\square$ & $\square$ & $\square$ & $\square$ \\
\hline d. Health organizations or groups (e.g. APDA) & $\square$ & $\square$ & $\square$ & $\square$ \\
\hline e. Charitable organizations & $\square$ & $\square$ & $\square$ & $\square$ \\
\hline f. Religious organizations and leaders & $\square$ & $\square$ & $\square$ & $\square$ \\
\hline g. Pharmaceutical companies & $\square$ & $\square$ & $\square$ & $\square$ \\
\hline h. Online forums and blogs & $\square$ & $\square$ & $\square$ & $\square$ \\
\hline
\end{tabular}

12. In general, how much would you trust information about food and nutrition from each of the following?

\begin{tabular}{|l|c|c|c|c|}
\hline & Not at all & A little & Some & A lot \\
\hline a. A doctor/pharmacist/health care provider & $\square$ & $\square$ & $\square$ & $\square$ \\
\hline b. Family or friends & $\square$ & $\square$ & $\square$ & $\square$ \\
\hline c. Government health agencies (e.g. FDA, NIH, CDC) & $\square$ & $\square$ & $\square$ & $\square$ \\
\hline $\begin{array}{l}\text { d. Health organizations/groups (APDA, Academy of } \\
\text { Nutrition and Dietetics (AND)) }\end{array}$ & $\square$ & $\square$ & $\square$ & $\square$ \\
\hline e. Religious organizations and leaders & $\square$ & $\square$ & $\square$ & $\square$ \\
\hline f. Pharmaceutical companies & $\square$ & $\square$ & $\square$ & $\square$ \\
\hline g. Online forums and health blogs & $\square$ & $\square$ & $\square$ & $\square$ \\
\hline h. Dietary programs (e.g. Weight Watchers, Paleo) & $\square$ & $\square$ & $\square$ & $\square$ \\
\hline
\end{tabular}

The following questions related to using the internet to access health information.

13. A. Do you ever go online to access the Internet/World Wide Web, or to send and receive e-mail? $\square$ Yes No

B. How often do you access the internet through each of the following?

\begin{tabular}{|l|c|c|c|c|}
\hline & Daily & Sometimes & Never & N/A \\
\hline a. Computer at home & $\square$ & $\square$ & $\square$ & $\square$ \\
\hline b. Computer at work & $\square$ & $\square$ & $\square$ & $\square$ \\
\hline c. Public computer (library/ community center) & $\square$ & $\square$ & $\square$ & $\square$ \\
\hline d. Mobile device (cellphone/smartphone) & $\square$ & $\square$ & $\square$ & $\square$ \\
\hline e. Tablet (iPad, Amazon Fire, other) & $\square$ & $\square$ & $\square$ & $\square$ \\
\hline f. Other (please specify): & $\square$ & $\square$ & $\square$ & $\square$ \\
\hline
\end{tabular}


14. A. Some people notice information about health on the Internet, even when they are not trying to find out about a health concern they have or someone in their family has. Have you read such health information on the Internet in the past 12 months?
Yes
No

B. About how often have you read this sort of information in the past 12 months.

Once a month or more

Less than once a month

15. Sometimes people use the Internet specifically for health-related reasons.

In the past 12 months, have you used the Internet for any of the following reasons?

\begin{tabular}{|l|c|c|}
\hline & Yes & No \\
\hline a. Looked for health or medical information for yourself & $\square$ & $\square$ \\
\hline b. Looked for health or medical information for someone else & $\square$ & $\square$ \\
\hline c. Looked for information about managing Parkinson's disease & $\square$ & $\square$ \\
\hline $\begin{array}{l}\text { d. Participated in an online forum or support group for people with similar } \\
\text { health or medical issue }\end{array}$ & $\square$ & $\square$ \\
\hline e. Used a website to help you with your diet, weight, or physical activity & $\square$ & $\square$ \\
\hline f. Looked for a health care provider & $\square$ & $\square$ \\
\hline $\begin{array}{l}\text { g. Downloaded heath information to a mobile device, such as an MP3 player, } \\
\text { cell phone, tablet computer, or electronic book device }\end{array}$ & $\square$ & $\square$ \\
\hline h. Shared health information on social media sites (Facebook, Twitter) & $\square$ & $\square$ \\
\hline i. Exchanged support about health concerns with family and friends & $\square$ & $\square$ \\
\hline $\begin{array}{l}\text { j. Kept track of personal health information such as care received, test results, } \\
\text { or upcoming medical appointments }\end{array}$ & $\square$ & $\square$ \\
\hline k. Watched a health-related video on YouTube & $\square$ & $\square$ \\
\hline
\end{tabular}




\section{APPENDIX R: Telephone Montreal Cognitive Assessment}

$\begin{array}{lr}\text { MONTREAL COGNITIVE ASSESSMENT / MoCA-BLIND } & \text { Name: } \\ \text { Version 7.1 Original Version } & \text { Sducation: } \\ & \text { Sex: } \\ & \text { Date of birth: } \\ \text { Date: }\end{array}$

\section{MEMORY}

Read list of words, subject must repeat them.

Do 2 trials even if 1 st trial is successful.

Do a recall after 5 minutes.

\section{ATTENTION}

Read list of digits (1 digit/sec.) Subject has to repeat them in the forward order

Subject has to repeat them in the backward order [ ] 742

Read list of letters. The subject must tap with his hand at each letter $A$. No point if $\geq 2$ errors

[ ] FBACMNAAJKLAFAKDEAAAJAMOFAAB

Serial 7 subtraction starting at 100
[ ] 93
[ ] 86
[ ] 79
[ ] 72
[ ] 65

4 or 5 correct subtractions: 3 pts, 2 or 3 correct: 2 pts, 1 correct: 1 pt, 0 correct: 0 pt

\section{LANGUAGE}

Repeat: I only know that John is the one to help today. [ ]

The cat always hid under the couch when dogs were in the room. [ ]

Fluency / Name maximum number of words in one minute that begin with the letter $F$.

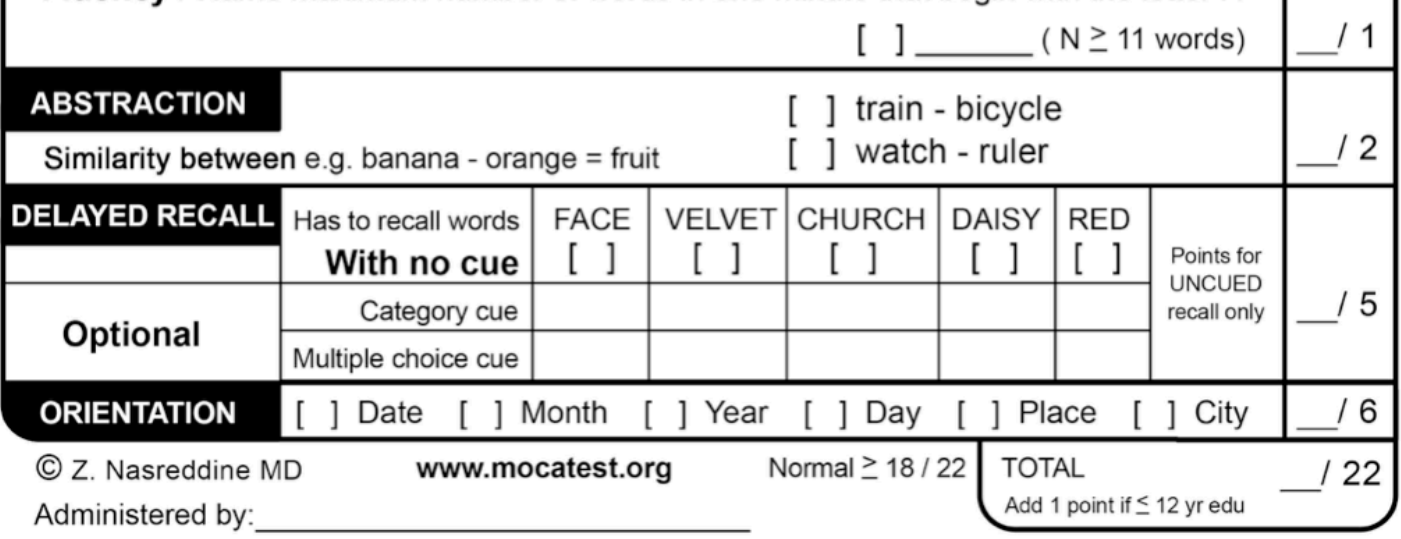




\section{APPENDIX S: EAT-10 Questionnaire}

EAT-10:

A Swallowing Screening Tool

Nestlé NutritionInstitute

$\begin{array}{lll}\text { LAST NAME } & \text { FIRST NAME } & \text { SEX } \\ \text { AGE } & \text { DATE } & \\ \text { OBJECTIVE: } & & \end{array}$

EAT-10 helps to measure swallowing difficulties.

It may be important for you to talk with your physician about treatment options for symptoms.

\section{A. INSTRUCTIONS:}

Answer each question by writing the number of points in the boxes.

To what extent do you experience the following problems?

1 My swallowing problem has caused me to lose weight.

6 Swallowing is painful.
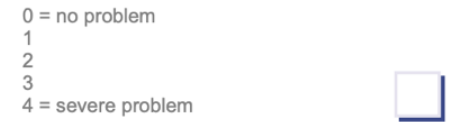

2 My swallowing problem interferes with my ability to go out for meals.

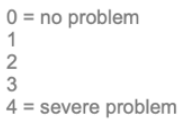

3 Swallowing liquids takes extra effort.

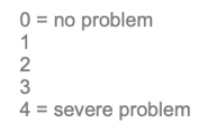

7 The pleasure of eating is affected by my swallowing.

$0=$ no problem

2

$4=$ severe problem

8 When I swallow food sticks in my throat.

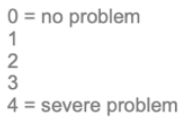

4 Swallowing solids takes extra effort.

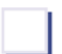

= no problem

$4=$ severe problem

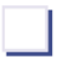

9 I cough when I eat.
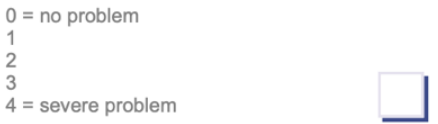

10 Swallowing is stressful.

5 Swallowing pills takes extra effort.

$0=$ no problem

$0=$ no problem

1

2

$4=$ severe problem
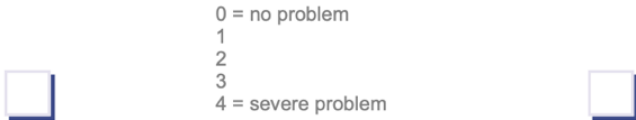

\section{B. SCORING:}

Add up the number of points and write your total score in the boxes.

Total Score (max. 40 points)

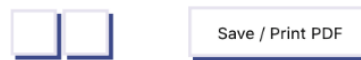

C. WHAT TO DO NEXT:

If the EAT-10 score is 3 or higher, you may have problems swallowing efficiently and safely. We recommend discussing the EAT-10 results with a physician.

Reference: The validity and reliability of EAT-10 has been determined.

Belafsky PC, Mouadeb DA, Rees CJ, Pryor JC, Postma GN, Allen J, Leonard RJ. Validity and Reliability of the Eating Assessment Tool (EAT-10). Annals of

www.nestlenutrition-institute.org 


\section{PDQ-39 QUESTIONNAIRE}

Please complete the following

Please tick one box for each question

Due to having Parkinson's disease, how often during the last month have you....

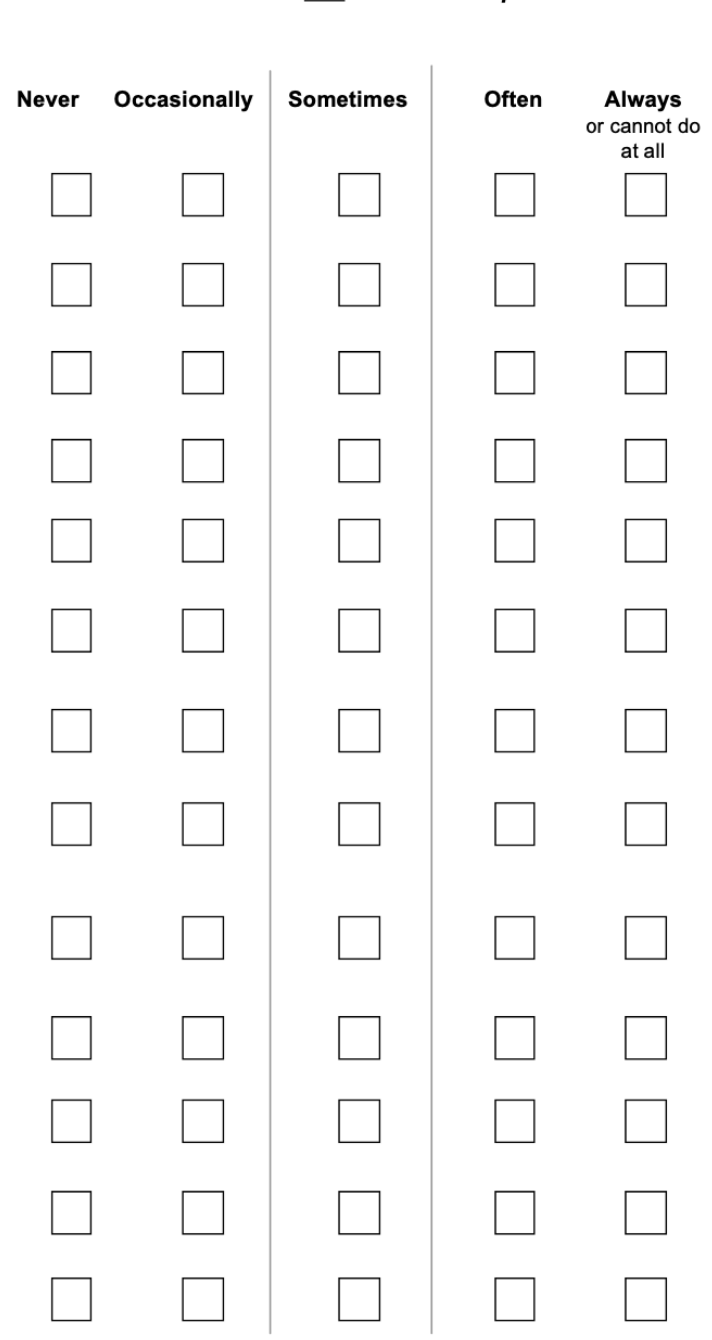

Had difficulty doing the leisure activities which you would like to do?

Had difficulty looking after your home, e.g. DIY, housework, cooking?

$3 \quad$ Had difficulty carrying bags of shopping?

$4 \quad$ Had problems walking half a mile?

$5 \quad$ Had problems walking 100 yards?

$6 \quad$ Had problems getting around the house as easily as you would like?

$7 \quad$ Had difficulty getting around in public?

$8 \quad$ Needed someone else to accompany you when you went out?

$9 \quad$ Felt frightened or worried about falling over in public?

10 Been confined to the house more than you would like?

$11 \quad$ Had difficulty washing yourself?

12 Had difficulty dressing yourself?

13 Had problems doing up your shoe laces?

Please check that you have ticked one box for each question before going on to the next page 
Due to having Parkinson's disease, how often during the last month

have you....

14

Had problems writing clearly?

15

Had difficulty cutting up your food?

16

Had difficulty holding a drink without spilling it?

17

Felt depressed?

18

19

20

21

22

23

24

support in the ways you need from your family or close friends?

Felt weepy or tearful?

Felt angry or bitter?

Felt anxious?

Felt worried about your

future?

you had to conceal your Parkinson's from people?

Avoided situations which involve eating or drinking in public?

Felt embarrassed in public due to having Parkinson's disease?

Felt worried by other people's reaction to you?

Had problems with your close personal relationships?

Lacked support in the ways you need from your spouse or partner?

Please tick one box for each question

Never

Occasionally Sometimes

Often

Always

or cannot do

at all

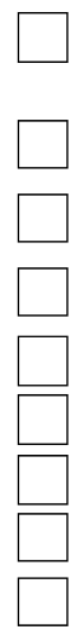

$\square$

$\square$

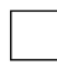

$\square$

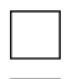

$\square$
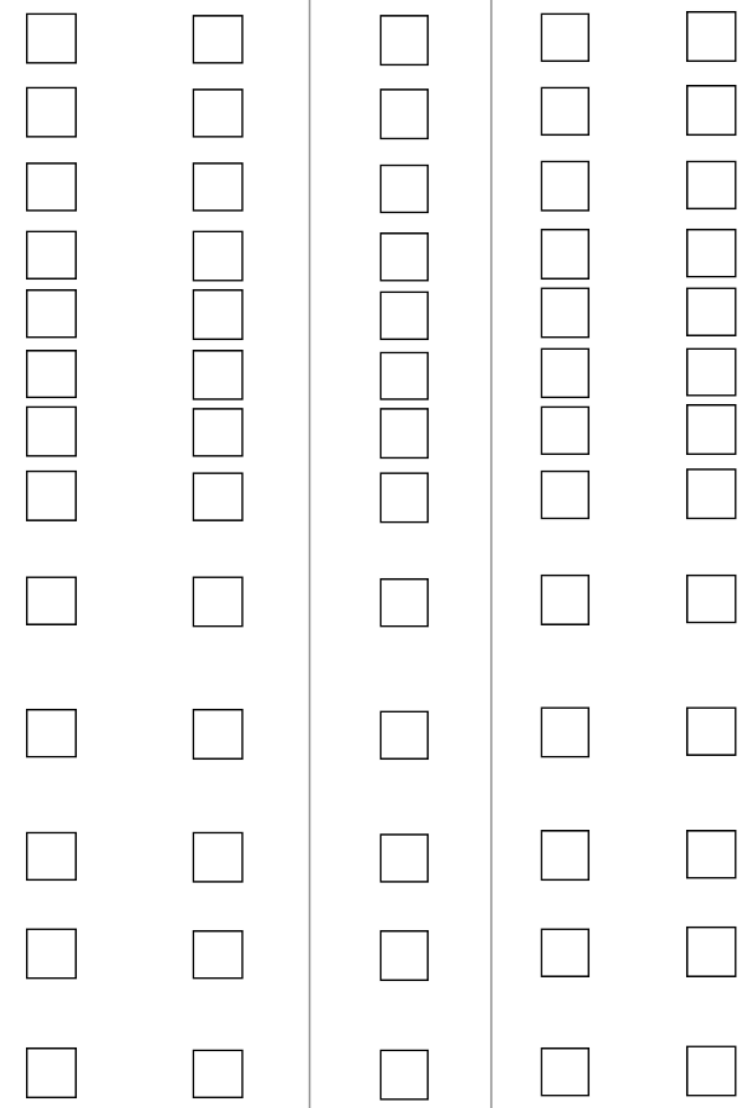

$$
\text { If you do not have a spouse or }
$$
partner tick here
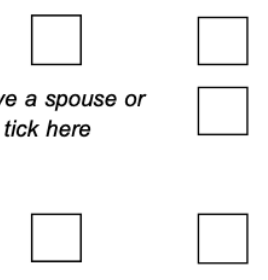

Please check that you have ticked one box for each question before going on to the next page 
Due to having Parkinson's disease, how often during the last month have you....

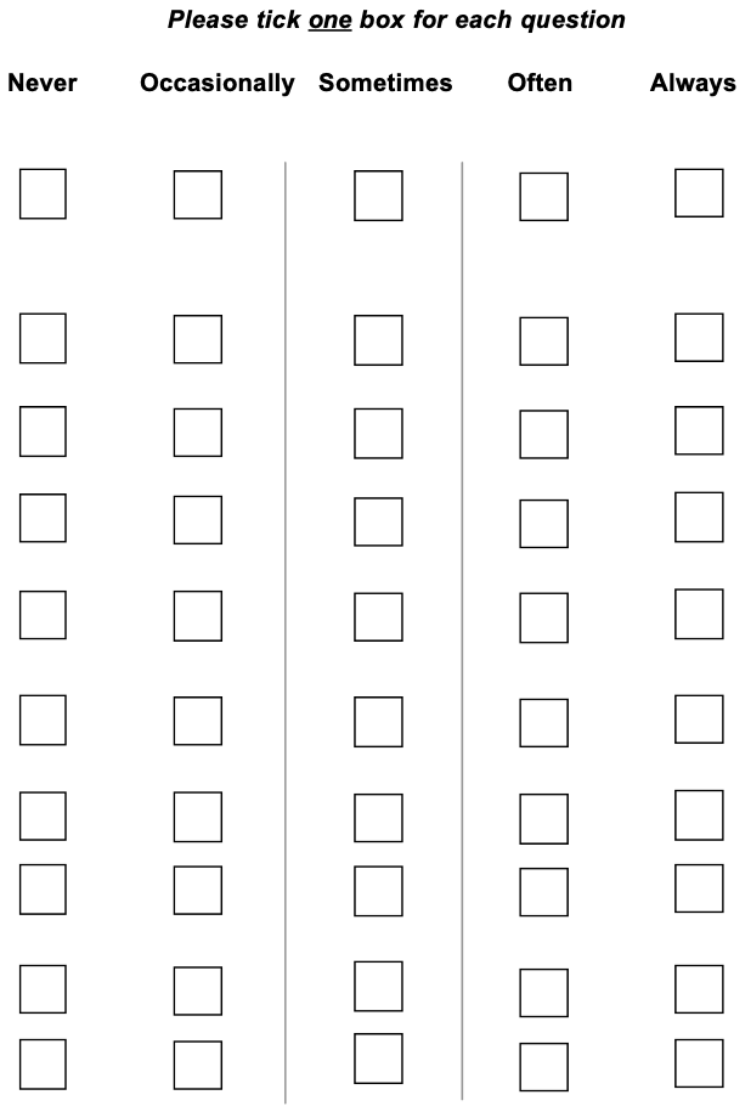

Please check that you have ticked one box for each question before going on to the next page

Thank you for completing the PDQ 39 questionnaire 
APPENDIX U: MCSI Questionnaire

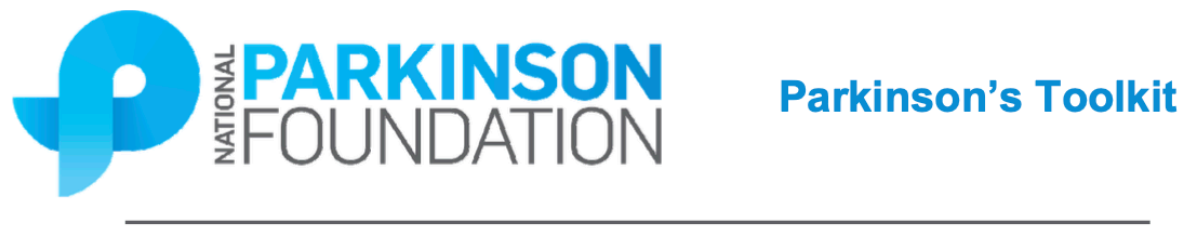

The Multidimensional Caregiver Strain Index

Instructions: Circle the number which most closely reflects in general your feelings about caring for your partner, relative, or friend.

\begin{tabular}{|c|c|c|c|c|c|}
\hline & Never & $\begin{array}{c}\text { A } \\
\text { little }\end{array}$ & Moderate & $\begin{array}{c}\text { A } \\
\text { Lot }\end{array}$ & $\begin{array}{l}\text { A great } \\
\text { Deal }\end{array}$ \\
\hline $\begin{array}{l}\text { 1. I feel I have less energy now that I am caring } \\
\text { for my spouse or family member. }\end{array}$ & 0 & 1 & 2 & 3 & 4 \\
\hline $\begin{array}{l}\text { 2. I fee; physically strained because of caring } \\
\text { for my spouse or family member. }\end{array}$ & 0 & 1 & 2 & 3 & 4 \\
\hline $\begin{array}{l}\text { 3. I feel that my physical health has suffered } \\
\text { because of caring for my spouse or family } \\
\text { member. }\end{array}$ & 0 & 1 & 2 & 3 & 4 \\
\hline $\begin{array}{l}\text { 4. I feel that my social life has suffered because } \\
\text { of caring for my spouse or family member. }\end{array}$ & 0 & 1 & 2 & 3 & 4 \\
\hline $\begin{array}{l}5 . \text { I have had to give upon vacations or trips } \\
\text { because of caring for my spouse or family } \\
\text { member. }\end{array}$ & 0 & 1 & 2 & 3 & 4 \\
\hline 6. I am able to go out when I want. & 0 & 1 & 2 & 3 & 4 \\
\hline $\begin{array}{l}\text { 7. I have had to make adjustments in my work } \\
\text { or personal schedule. }\end{array}$ & 0 & 1 & 2 & 3 & 4 \\
\hline $\begin{array}{l}\text { 8. Caring for/ providing help for my spouse or } \\
\text { family member is a financial strain. }\end{array}$ & 0 & 1 & 2 & 3 & 4 \\
\hline $\begin{array}{l}\text { 9. I resent the extra cost of caring for my } \\
\text { spouse or family member. }\end{array}$ & 0 & 1 & 2 & 3 & 4 \\
\hline $\begin{array}{l}\text { 10. I have enough time to do the things I need } \\
\text { to do (Such as chores and helping). }\end{array}$ & 0 & 1 & 2 & 3 & 4 \\
\hline 11. I have a lot of time to myself. & 0 & 1 & 2 & 3 & 4 \\
\hline $\begin{array}{l}\text { 12. I feel resentful toward my spouse or family } \\
\text { member. }\end{array}$ & 0 & 1 & 2 & 3 & 4 \\
\hline $\begin{array}{l}\text { 13. I feel angry toward my spouse or family } \\
\text { member. }\end{array}$ & 0 & 1 & 2 & 3 & 4 \\
\hline $\begin{array}{l}\text { 14. I feel pleased about my relationship with my } \\
\text { spouse or family member. }\end{array}$ & 0 & 1 & 2 & 3 & 4 \\
\hline $\begin{array}{l}\text { 15. My relationship with my spouse or family } \\
\text { member is strained. }\end{array}$ & 0 & 1 & 2 & 3 & 4 \\
\hline $\begin{array}{l}\text { 16. I am glad that I can provide care for my } \\
\text { spouse or family member. }\end{array}$ & 0 & 1 & 2 & 3 & 4 \\
\hline
\end{tabular}

Page 1 of 2

HELPLINE: English /Español

1.800.4PD.INFO (473-4636)

Get your PD questions answered. 


\begin{tabular}{|c|c|c|c|c|c|}
\hline $\begin{array}{l}\text { 17. I feel my spouse or family member tries to } \\
\text { manipulate me. }\end{array}$ & 0 & 1 & 2 & 3 & 4 \\
\hline $\begin{array}{l}\text { 18. I feel that my spouse or family member is } \\
\text { overly demanding }\end{array}$ & 0 & 1 & 2 & 3 & 4 \\
\hline
\end{tabular}

Scoring: Reverse code questions $6,10,11,14$, and 16 for a total score.

Adapted from: Stull D. (1996) - The Multidimensional Caregiver strain Index (MCSI): Its Measurement and Structure. Journal of Clinical Geropsychology, Vol. 2, (3), 175-196.

Page 2 of 2 


\title{
APPENDIX V: Past Medical History Questionnaire - Person with Parkinson's Disease
}

\author{
Tailoring Digital Health to Improve the Nutrition and Health of People with Parkinson's Disease \\ Ingrid Lofgren, PhD, Principal Investigator \\ Leslie Mahler, PhD, CCC-SLP, Co-Investigator \\ Version 1: 4.4.2018 \\ Interview for Person with Parkinson's disease
}

Name:

Initials:

ID\#:

Name of Interviewer:

Date:

Participant Address:

Phone Number:

Email:

Emergency contact name \& phone:

DIRECTIONS: Read the following questions out loud to each prospective volunteer and record the answers. Any answers that require clarification should be written in the space below the question or on the back of the sheet. Indicate whether any follow-up is necessary or if any referrals are appropriate.

\section{Overview}

Date of Birth:

Age:

Gender:

\section{Parkinson's disease:}

What is your neurological diagnosis?

When were you diagnosed (month/year)?

What were your symptoms at that time?

What are your symptoms now?

What treatments have you had for Parkinson's disease?

Do you currently take any medications to manage your Parkinson's disease?

If so, which ones?

For how long?

How many times/day?

Page 1 of 6 


\section{Gastrointestinal and Diet:}

- What is your diet like?

- Do you have any food allergies?

- Do you have any known gastrointestinal conditions (reflux, celiac disease, irritable bowel syndrome, diverticular disease, etc.)

olf yes, when were you diagnosed?

o How do you manage this condition? (medication, diet, etc.)

- Do you experience any other symptoms that may impact dietary intake (bloating, constipation, loose stools, distended stomach, nausea, vomiting, diarrhea, gas)

oIf yes, for how long and how frequently?

$\circ$ How do you manage this?

- Do you take any dietary or herbal supplements? If so, which ones and for what reason?

- Have you experienced any weight change in the last year?

o If so, how recently?

o Intentional or Unintentional?

o What is your usual body weight?

Speech \& Swallowing:

Are you experiencing any symptoms of a swallowing disorder?

If yes, what is the problem with your swallowing?

What would you say is your most significant problem with speech or swallowing today?

Did you experience any changes in your speech or swallowing before your diagnosis?

Does medication affect your speech or voice or swallowing? If yes, in what way?

Page 2 of 6 
Musculoskeletal system:

Has your doctor ever told you that you have: (check all that apply)

- _ Osteoarthritis or degenerative arthritis

- _ Rheumatoid arthritis

- ___ Ankylosing spondylitis

- Osteoporosis

- __ Unknown or other type of arthritis

- _ _ Any other disease of joint or muscle

\section{Cardiovascular system:}

- Has any family member had a heart attack prior to the age of 55 ?

- If so, how are they related to you?

- Have you ever had frequent cramping in your legs while resting?

- If yes, is it a current problem?

- Have you ever had pain or cramping in your legs while walking?

- If yes, is it a current problem?

- If yes, is this pain relieved by rest or by discontinuing walking?

- Have you ever been told that you have high blood pressure?

- If yes, what was the date of onset?

○ Were you given any medications and which?

- Did a doctor ever tell you that you had a heart problem?

- If yes: What was the date of onset?

- What did the doctor call it?

$\circ$ Were you given any medications?

- Do you have any history of high cholesterol as evidenced by previous blood lipid tests?

- If yes, were you given any medications and if so, which one(s)?

\section{Endocrine system:}

Have you ever had any of the following: Thyroid problems, adrenal problems, diabetes mellitus?

- If yes to diabetes, which type? Type 1 or Type $2 \quad$ (circle one)

Date of onset

Are/were you on any medication, or is it diet/exercise controlled?

\section{Neurological system:}

- Do you have any neurological conditions or a history of a neurological condition?

Page 3 of 6 
- Do you have any significant problems with your memory? (circle all that apply)

○ When answering the telephone, do you recall what you were doing before it rang?

- Can you give the directions to your house/apartment?

- Can you keep appointments without a reminder?

- Can you remember what clothes you wore yesterday?

- Is it difficult for you to pay attention long enough to finish a task?

- Do you have any difficulty reading?

- Do you have any difficulty writing?

- Any problems with vision other than corrective lens changes?

- If yes, which of the following conditions- blindness, temporary loss of vision, double vision, glaucoma, cataract, macular degeneration or others?

Do you have any of the following? (check all that apply)

- ___ Vertigo (a feeling of spinning, or unsteadiness)

- _ _ Seizure or convulsions?

- _ Migraine or severe headaches?

- __ Paralysis of arm or leg?

- _ _ A head injury with loss of consciousness?

- __ Pain, numbness or tingling in your limbs?

- __ Pain in your lower back?

- __ Do you have pain in any part of body including headaches while exercising?

- _ _ Have you been told that you have a peripheral neuropathy?

- Tremors?

- Do you have problems with walking? If yes,

- Do you fall frequently and how often?

- Is your walking problem related to pain, weakness or loss of balance?

Have you ever had an operation on skull or brain? If so, when? 
Previous Treatments:

Have you had previous speech/swallow treatment, occupational therapy or physical therapy?

If yes, please describe (when, what):

Was it beneficial and what changes did you notice?

Other Lifestyle Factors:

Have you experienced any changes in your mood? If so, please describe.

Do you participate in physical activity/exercise? If so what type and of how often? (hours\&days/week)

Do you currently smoke cigarettes?

If you have a history of smoking cigarettes, when did you quit?

Do you have any other health problems or conditions that would affect your health?

Are there any other medications or supplements you take that we did not discuss in this interview?

How would you describe your health? (circle one)

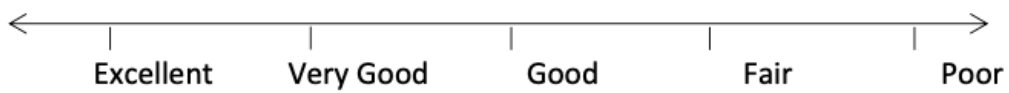

Employment:

Are you currently employed? Type of employment and hours per week

Education: Which best describes your education?

- _ L Less than a HS Diploma

- _ GED/HS Diploma

- _ Some College

- _ Bachelor's Degree

- _ Associates Degree

- _ Post-Baccalaureate Degree

- Technical/Trade School

-Continue on Next Page-

Page 5 of 6 
Race/Ethnicity:

Are you Hispanic or Latino?

What race/ethnicity do you identify with? (Chose one)

White/Caucasian African American Asian American Indian/Alaskan

Native Native Hawaiian/Pacific Islander Middle Eastern

I prefer not to answer Other

The follow set of questions relate to relationships in your life. FRIENDSHIPS: When answering the items below, consider all friends including those who live in your neighborhood.

\begin{tabular}{|l|c|c|c|c|c|c|}
\hline & None & One & Two & 3-4 & $5-8$ & $9+$ \\
\hline $\begin{array}{l}\text { How many of your friends do you see or hear from at least } \\
\text { once a month? }\end{array}$ & $\square$ & $\square$ & $\square$ & $\square$ & $\square$ & $\square$ \\
\hline $\begin{array}{l}\text { How many friends do you feel at ease with that you can talk } \\
\text { about private matters? }\end{array}$ & $\square$ & $\square$ & $\square$ & $\square$ & $\square$ & $\square$ \\
\hline $\begin{array}{l}\text { How many friends do you feel close to such that you could call } \\
\text { on them for help? }\end{array}$ & $\square$ & $\square$ & $\square$ & $\square$ & $\square$ & $\square$ \\
\hline
\end{tabular}

FAMILY: When answering the items below, consider all of the people to whom you are related by birth, marriage, adoption, etc. (include spouse/partner)

\begin{tabular}{|l|l|l|l|l|l|l|}
\hline & None & One & Two & $3-4$ & $5-8$ & $9+$ \\
\hline $\begin{array}{l}\text { How many of relatives do you see or hear from at least once a } \\
\text { month? }\end{array}$ & $\square$ & $\square$ & $\square$ & $\square$ & $\square$ & $\square$ \\
\hline $\begin{array}{l}\text { How many relatives do you feel at ease with that you can talk } \\
\text { about private matters? }\end{array}$ & $\square$ & $\square$ & $\square$ & $\square$ & $\square$ & $\square$ \\
\hline $\begin{array}{l}\text { How many relatives do you feel close to such that you could } \\
\text { call on them for help? }\end{array}$ & $\square$ & $\square$ & $\square$ & $\square$ & $\square$ & $\square$ \\
\hline
\end{tabular}

RELATIONSHIPS: Please respond to the following questions below. How much do you agree or disagree with the following?

\begin{tabular}{|l|c|c|c|c|c|}
\hline & $\begin{array}{c}\text { Strongly } \\
\text { Disagree }\end{array}$ & Disagree & Neutral & Agree & $\begin{array}{c}\text { Strongly } \\
\text { Agree }\end{array}$ \\
\hline I am content with my friendships and relationships. & $\square$ & $\square$ & $\square$ & $\square$ & $\square$ \\
\hline $\begin{array}{l}\text { I have enough people I feel comfortable asking for } \\
\text { help at any time. }\end{array}$ & $\square$ & $\square$ & $\square$ & $\square$ & $\square$ \\
\hline $\begin{array}{l}\text { My relationships are as satisfying as I would want } \\
\text { them to be. }\end{array}$ & $\square$ & $\square$ & $\square$ & $\square$ & $\square$ \\
\hline
\end{tabular}

\#\#\#-END-\#\#\#

Page 6 of 6 


\title{
APPENDIX W: Past Medical History Questionnaire for Caregiver
}

\author{
Tailoring Digital Health to Improve the Nutrition and Health of People with Parkinson's Disease \\ Ingrid Lofgren, PhD, Principal Investigator \\ Leslie Mahler, PhD, CCC-SLP, Principal Investigator
}

Version 1: 5.4.2018

Interview for Caregiver of Person with PD

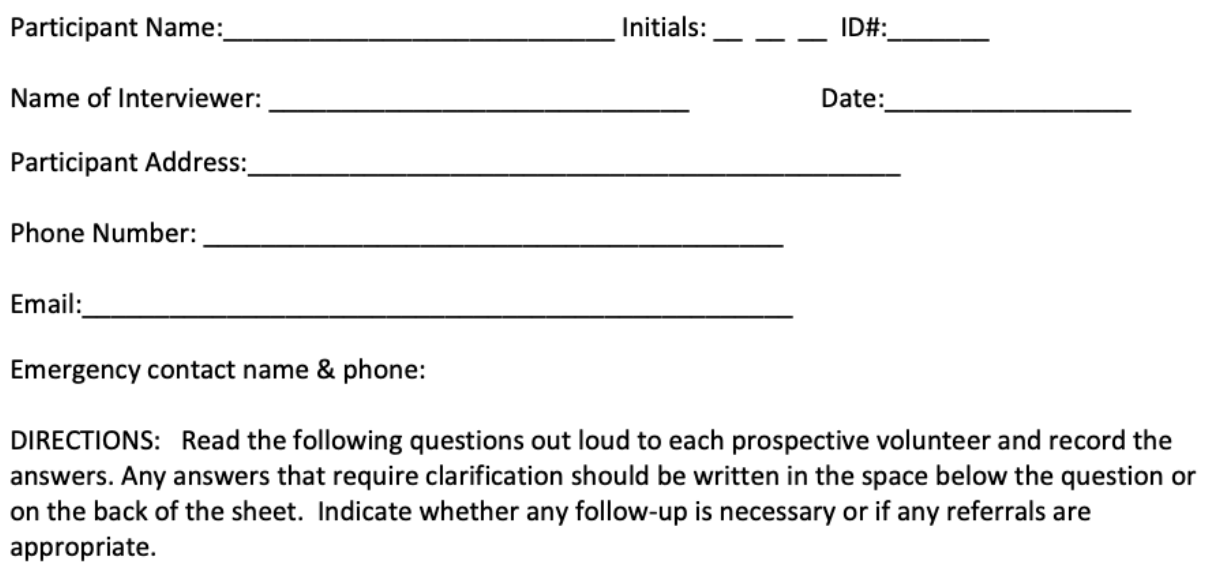

DIRECTIONS: Read the following questions out loud to each prospective volunteer and record the answers. Any answers that require clarification should be written in the space below the question or on the back of the sheet. Indicate whether any follow-up is necessary or if any referrals are appropriate.

\section{Overview:}

Date of Birth:

Age:

Gender:

\section{Gastrointestinal and Diet:}

- What is your diet like?

- Do you have any food allergies/intolerances?

- Do you have any known gastrointestinal conditions (reflux, celiac disease, irritable bowel syndrome, diverticular disease, etc.)

- If yes, when were you diagnosed?

- How do you manage this condition? (medication, diet, etc.)

- Do you experience any other symptoms that may impact dietary intake (bloating, constipation, loose stools, distended stomach, nausea, vomiting, diarrhea, gas)?

- If yes, for how long and how frequently?

$\circ$ How do you manage this? 
- Do you take any dietary or herbal supplements? If so, which ones and for what reason?

- Have you experienced any weight change in the last year?
- If so, how recently?
○ Intentional or Unintentional?
○ What is your usual body weight?

\section{Speech \& Swallowing}

Are you experiencing any symptoms of a swallowing disorder?

If yes, what is the problem with your swallowing?

\section{Musculoskeletal system:}

Has your doctor ever told you that you have: (check all that apply)

- __ Osteoarthritis or degenerative arthritis

- _ Rheumatoid arthritis

- __ Osteoporosis

- __ Ankylosing spondylitis

- __ Unknown or other type of arthritis

- __ Any other disease of joint or muscle:

\section{Cardiovascular system:}

- Has any family member had a heart attack prior to the age of 55?

- If so, how are they related to you?

- Have you ever had frequent cramping in your legs while resting?

- If yes, is it a current problem?

- Have you ever had pain or cramping in your legs while walking?

- If yes, is it a current problem?

- If yes, is this pain relieved by rest or by discontinuing walking?

- Have you ever been told that you have high blood pressure?

- If yes, what was the date of onset?

Page 2 of 6 
- Were you given any medications and which?

- Did a doctor ever tell you that you had a heart problem?

- If yes: What was the date of onset?

- What did the doctor call it?

○ Were you given any medications?

- Do you have any history of high cholesterol as evidenced by previous blood lipid tests?

- If yes, were you given any medications and if so, which one(s)?

\section{Endocrine system:}

Have you ever had any of the following: Thyroid problems, adrenal problems, diabetes mellitus?

- If yes to diabetes, which type? Type 1 or Type 2

Date of onset

Are/were you on any medication, or is it diet/exercise controlled?

\section{Neurological system:}

- Do you have any neurological conditions or a history of a neurological condition?

- Do you have any significant problems with your memory? (circle all that apply)

- When answering the telephone, do you recall what you were doing before it rang?

- Can you give the directions to your house/apartment?

○ Can you keep appointments without a reminder?

○ Can you remember what clothes you wore yesterday?

- Is it difficult for you to pay attention long enough to finish a task?

- Do you have any difficulty reading?

- Do you have any difficulty writing?

- Any problems with vision other than corrective lens changes?

- If yes, which of the following conditions- Blindness, temporary loss

Page 3 of 6 
of vision, double vision, glaucoma, cataract, macular degeneration or others?

Do you have any of the following? (check all that apply)

- _ _ Vertigo (a feeling of spinning, or unsteadiness)

- __ Seizure or convulsions?

- __ Migraine or severe headaches?

- _ Paralysis of arm or leg?

- _ _ A head injury with loss of consciousness?

- __ Pain, numbness or tingling in your limbs?

- _ Pain in your lower back?

- _ Do you have pain in any part of body including headaches while exercising?

- _ Have you been told that you have a peripheral neuropathy?

- _ Tremors?

- __ Problems with walking? If yes,

- Do you fall frequently and how often?

- Is your walking problem related to pain, weakness or loss of balance?

Have you ever had an operation on skull or brain? If so, when?

Have you ever had meningitis or "brain fever"?

\section{Previous Treatment}

Have you had previous speech or swallow treatment, occupational therapy or physical therapy?

If yes, please describe (when, what):

Was it beneficial?

If yes, what changes did you notice?

\section{Other Lifestyle Factors}

Have you experienced any changes in your mood? If so, please describe.

Page 4 of 6 
Do you participate in physical activity/exercise? If so what type and of how often? (hours\&days/week)

Do you currently smoke cigarettes?

If you have a history of smoking cigarettes, when did you quit?

Do you have any other health problems or conditions that would affect your health?

Are there any other medications or supplements you take that we did not discuss in this interview?

How would you describe your health? (circle one)

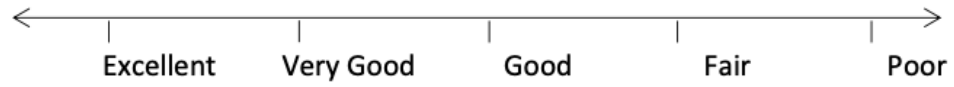

Education: Which bests describes your education?

- _ Less than a HS Diploma

- _ GED/HS Diploma

- $\quad$ Some College

- _ Bachelor's Degree

- $\quad$ Associates Degree

- _ Post-Baccalaureate Degree

- Technical/Trade School

Employment:

Are you currently employed?

Type of employment and hours per week

\section{Race/Ethnicity:}

Are you Hispanic or Latino?

What race/ethnicity do you identify with? (Chose one)

White/Caucasian African American Asian American Indian/Alaskan

Native__ Native Hawaiian/Pacific Islander Middle Eastern

I prefer not to answer Other

\section{-Continue on Next Page-}

Page 5 of 6 
The follow set of questions relate to relationships in your life.

FRIENDSHIPS: When answering the items below, consider all friends including those who live in your neighborhood.

\begin{tabular}{|l|c|c|c|c|c|c|}
\hline & None & One & Two & $3-4$ & $5-8$ & $9+$ \\
\hline $\begin{array}{l}\text { How many of your friends do you see or hear from at least } \\
\text { once a month? }\end{array}$ & $\square$ & $\square$ & $\square$ & $\square$ & $\square$ & $\square$ \\
\hline $\begin{array}{l}\text { How many friends do you feel at ease with that you can talk } \\
\text { about private matters? }\end{array}$ & $\square$ & $\square$ & $\square$ & $\square$ & $\square$ & $\square$ \\
\hline $\begin{array}{l}\text { How many friends do you feel close to such that you could call } \\
\text { on them for help? }\end{array}$ & $\square$ & $\square$ & $\square$ & $\square$ & $\square$ & $\square$ \\
\hline
\end{tabular}

FAMILY: When answering the items below, consider all of the people to whom you are related by birth, marriage, adoption, etc. (include spouse/partner)

\begin{tabular}{|l|c|c|c|c|c|c|}
\hline $\begin{array}{l}\text { How many of relatives do you see or hear from at least once a } \\
\text { month? }\end{array}$ & None & One & Two & 3-4 & $5-8$ & $9+$ \\
\hline $\begin{array}{l}\text { How many relatives do you feel at ease with that you can talk } \\
\text { about private matters? }\end{array}$ & $\square$ & $\square$ & $\square$ & $\square$ & $\square$ \\
\hline $\begin{array}{l}\text { How many relatives do you feel close to such that you could } \\
\text { call on them for help? }\end{array}$ & $\square$ & $\square$ & $\square$ & $\square$ & $\square$ & $\square$ \\
\hline
\end{tabular}

RELATIONSHIPS: Please respond to the following questions below. How much do you agree or disagree with the following?

\begin{tabular}{|l|c|c|c|c|c|}
\hline & $\begin{array}{c}\text { Strongly } \\
\text { Disagree }\end{array}$ & Disagree & Neutral & Agree & $\begin{array}{c}\text { Strongly } \\
\text { Agree }\end{array}$ \\
\hline I am content with my friendships and relationships. & $\square$ & $\square$ & $\square$ & $\square$ & $\square$ \\
\hline $\begin{array}{l}\text { I have enough people I feel comfortable asking for } \\
\text { help at any time. }\end{array}$ & $\square$ & $\square$ & $\square$ & $\square$ & $\square$ \\
\hline $\begin{array}{l}\text { My relationships are as satisfying as I would want } \\
\text { them to be. }\end{array}$ & $\square$ & $\square$ & $\square$ & $\square$ & $\square$ \\
\hline
\end{tabular}




\section{APPENDIX X: Request for Personal Health Information}

THE UNIVERSITY

OF RHODE ISLAND

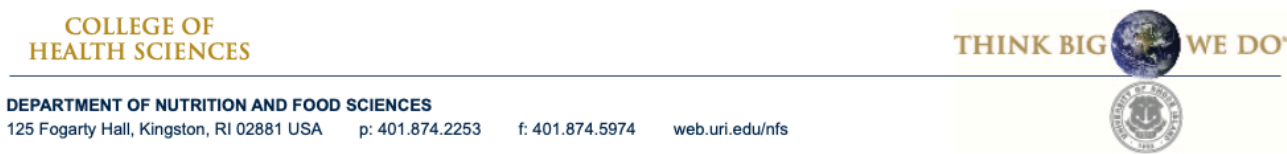

\section{AUTHORIZATION FOR USE OR DISCLOSURE OF PROTECTED HEALTH INFORMATION} FOR RESEARCH: For Person with Parkinson's Disease

The University of Rhode Island

Department of Nutrition and Food Sciences

Fogarty Hall

41 Lower College Rd

Kingston RI, 02881

Tailoring Digital Health to Improve the Nutrition and Health of People with Parkinson's Disease

The privacy law, Health Insurance Portability \& Accountability Act (HIPAA) protects individually identifiable health information. The privacy law requires that an investigator explain in detail what information will be obtained during a study and how that information will be used, and with whom it will be shared.

You have been asked to participate in the above named study. The protected health information that may be used and disclosed includes:

- Date of Parkinson's Diagnosis

- Medication dosing and timing for Parkinson's

- Treatments for Parkinson's disease, such as Deep Brain Stimulation

- Stage of Parkinson's as measured by Hoehn Yahr and Unified Parkinson's Disease Rating Scale

The investigators may use and disclose your protected health information until the end of the study (August 2020). They will use and/or share this information with:

The University of Rhode Island Institutional Review Board 
Government Agencies when required by law

You do not have to sign this authorization. If you do sign, you may end your participation by notifying the investigator (name and telephone \# of investigator). Withdrawal of authorization will not affect treatment, payment or enrollment in any health plans or affect your eligibility for benefits. When you withdraw authorization investigators may only use and disclose the protected health information already collected for this research study. Withdrawal of authorization also means that you may not be allowed to participate in the study.

The investigator will respect the confidentiality of the health information, however, should the health information be disclosed by the investigator, to someone outside of this study, it may no longer be protected by the HIPAA regulation.

Signing your name at the bottom of this form means that you have read or listened to what it says and you understand it. Signing this form also means that you agree to authorize the use and disclosure of personal health information. You will be given a copy of this form after you have signed it.

Signature of participant

Typed/printed Name

Date
Signature of Researcher

Typed/printed Name 